\title{
Hermeneutic Constructivism: An Ontology for Qualitative Research
}

\begin{abstract}
BLAKE S. PECK
$\mathrm{RN}, \mathrm{BN}$ (Hon)

This thesis is submitted in total fulfillment of the requirements for the degree of Doctor of Philosophy
\end{abstract}

School of Education and Arts

University of Ballarat

PO Box 663

University Drive, Mount Helen

Ballarat, Victoria 3353

Australia 


\begin{abstract}
This thesis begins with contemporary qualitative research, where the extent of what is understood about human experience is reduced to the representations constructed by researchers. In this situation, where the qualitative researcher has a monopoly on the representation produced, there is no scope for a consideration of the expressive nature of language - in particular, the way that language discloses the world differently for each individual person. Thus, the aim of this thesis is to develop a theoretical approach for understanding the personal realities of the people involved in qualitative research that reinstates the centrality of the dialogic in understanding.

The argument that supports this theoretical development is framed in two different ways. Firstly, the practices pervasive to contemporary qualitative research necessitate that a researcher assume a naïve view of language and meaning. Secondly, an accord on criteria with which to assess the worth of qualitative research remains elusive, emphasising a disregard for the representation of a real. These arguments inform a view that qualitative research is unable to represent the idiographic aspects of human experience. As such, these arguments culminate in a call for a new theoretical approach that reinstates the individual as the rightful unit of consideration in qualitative research.
\end{abstract}

In response to these provocative calls, this thesis initially draws on Kelly’s Personal Construct Psychology given its recognition of the idiographic aspects of being human.

However, Kelly's consideration of language is inadequate, opening to challenge the theoretical tenability of his position. As a model that informs qualitative research, an inherently linguistic endeavour, the Psychology of Personal Constructs is insufficient. In order to overcome these theoretical inadequacies, Gadamer's Hermeneutic Phenomenology provides a productive foundation from which to re-envision the core principles of Personal Construct Psychology. The tenets of this re-envisioning have been set-down as a theoretical position referred to by this thesis as Hermeneutic Constructivism. This theoretical position is able to inform a new qualitative research that makes possible an exploration of a person's experience at a deeper level of abstraction. 


\section{STATEMENT OF AUTHORSHIP}

Except where explicit reference is made in the text of the thesis, this thesis contains no material published elsewhere or extracted in whole or in part from a thesis by which I have qualified for or been awarded another degree or diploma. No other person's work has been relied upon or used without due acknowledgement in the main text and bibliography of the thesis.

Signed:

Signed:

Dated:

Dated:

Blake S. Peck

Dr. Jane Mummery

Candidate

Principal Supervisor 


\section{ACKNOWLEDGEMENTS}

I would like to acknowledge the role of my principal supervisor, Dr. Jane Mummery, who has steadfastly been with me across this theoretical journey, and thank her for sharing with me her depth of knowledge and appreciation of philosophical contemplation. Our working relationship has produced some inspired thought and I am sure it will be the edifice of many ongoing discussion in the future. To Dr. Angus McLachlan, my associate supervisor, I would like to express thanks for being a productively critical voice along the way.

Special mention also goes to my dear friend and colleague, Dr. Bernie Whitaker, who in the early stages of this thesis was instrumental in helping me see the world very differently, and for this I am grateful. As well as Bernie, other people also contributed their time, and thought, by reading drafts and providing commentary Jennifer Roe, Russell Collins, Dr. Petah Gibbs, Dr. Philip Warelow and Professor John Macdonald - to these people I am also grateful.

To my parents, I thank you for providing me with a childhood that was free from dogmatic thought, an upbringing that inspired a sense of curiosity and gave me an opportunity to make and take my own chances by challenging the status quo. These foundations have been instrumental in setting me off on this journey.

Finally, I would specially like to thank my wife Bronwyn. It is to you that I am most indebted. You have given me incredible support in so many ways, through the times that have been hard, and through the times that have been easier, you have tolerated the early mornings as well as the late nights, and have been with me for the entire journey. For this I am grateful. Thanks must also go to my children Abbie and Olivia. Although you have not been able to help me directly with the thesis itself, you have changed my life in so many ways, and for this I am eternally fortunate. 


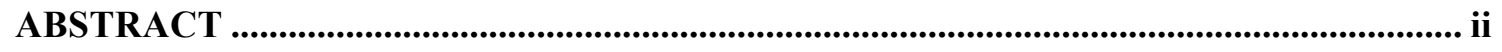

STATEMENT OF AUTHORSHIP........................................................................................

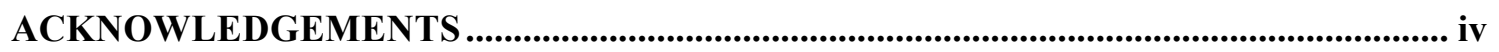

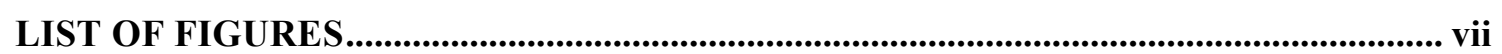

CHAPTER 1 - SIGNIFICANCE AND ORGANISATION ....................................................1

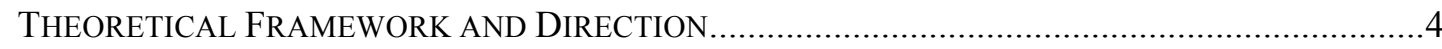

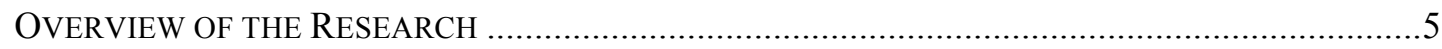

CHAPTER 2 - REPRESENTATION IN QUALITATIVE RESEARCH ..............................8

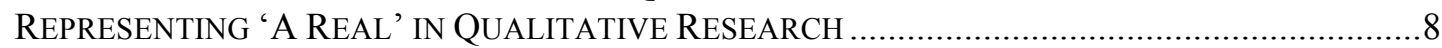

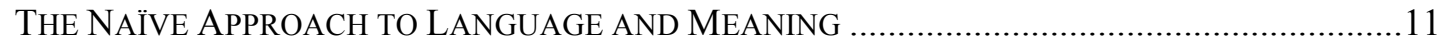

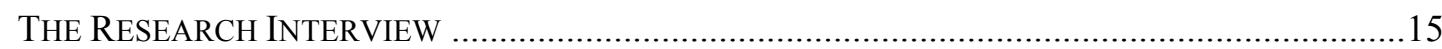

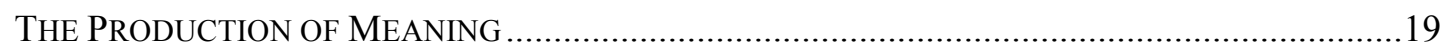

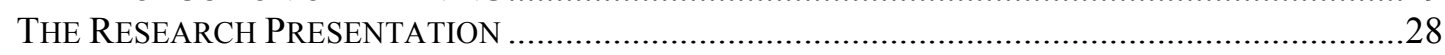

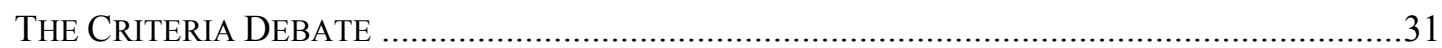

THE INDIVIDUAL AS FOCUS OF THE QUALITATIVE ENDEAVOUR...........................................44

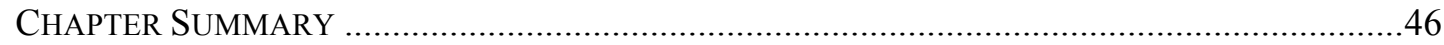

CHAPTER 3 - THE IDIOGRAPHIC PERSPECTIVE ..................................................48

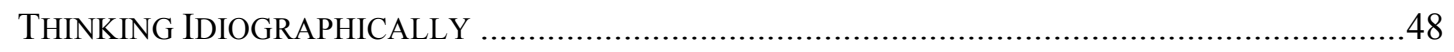

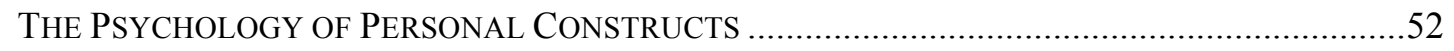

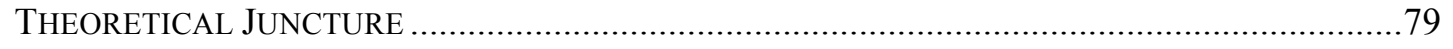

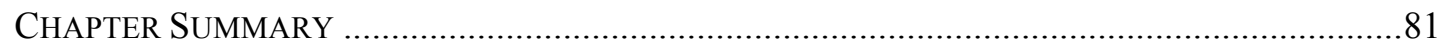

CHAPTER 4 - KELLY'S NAÏVE VIEW OF LANGUAGE .................................................83

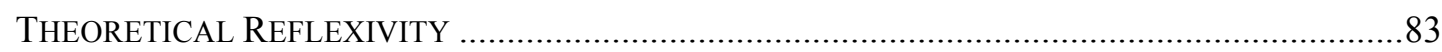

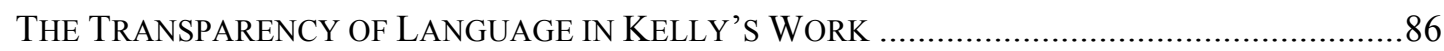

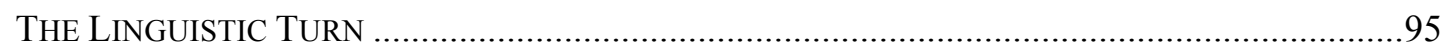

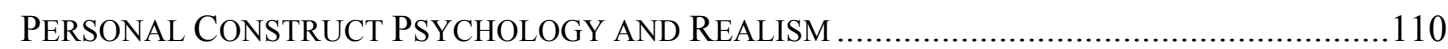

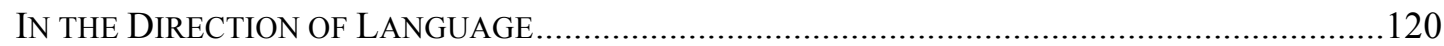

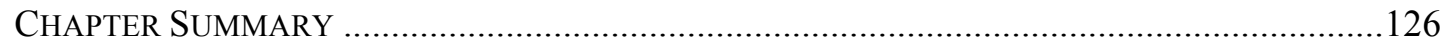

CHAPTER 5 - LANGUAGE AS THE MISSING MEDIUM.................................................128

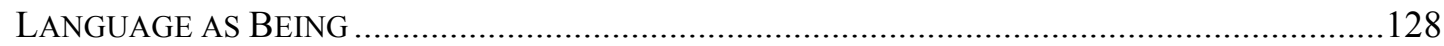

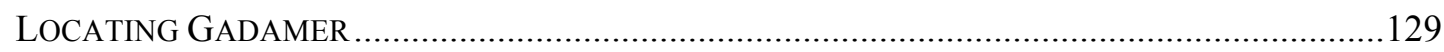

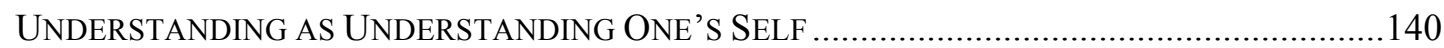

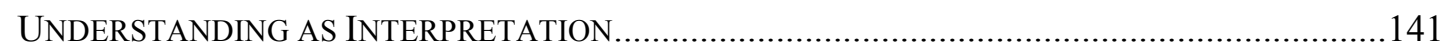

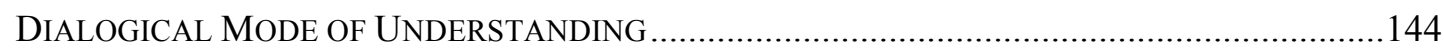

QUESTION AND ANSWER AS THE FoUNDATION OF DIALOGUE ............................................146

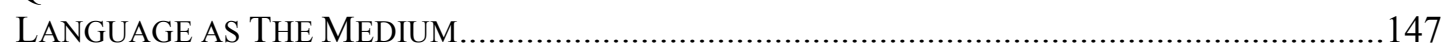

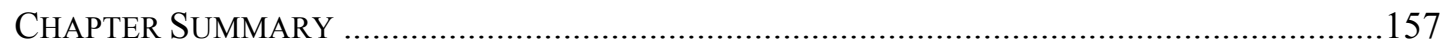

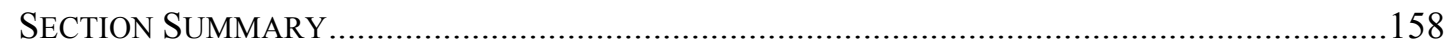

CHAPTER 6 - THE IDIOGRAPHIC WITHIN A LANGUAGED WORLD.......................160

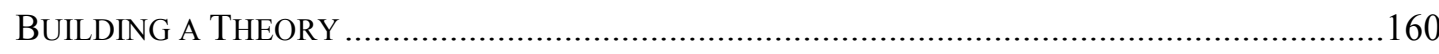

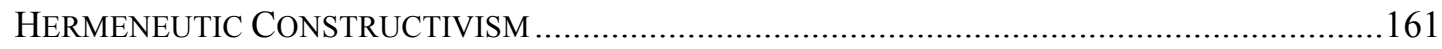

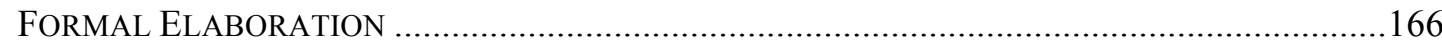

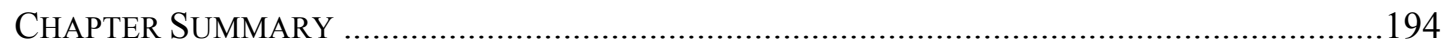


CHAPTER 7 - HERMENEUTIC CONSTRUCTIVISM: AN ELABORATION ...............196

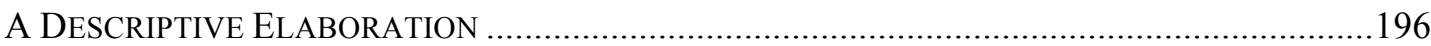

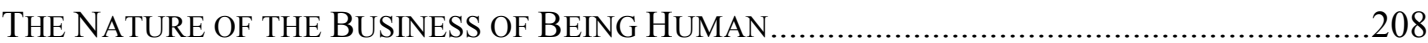

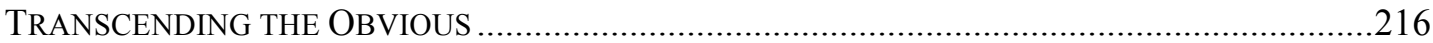

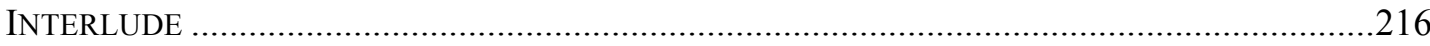

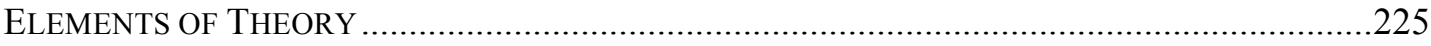

A HERMENEUTIC CONSTRUCTIVIST LENS ON PERSONAL CONSTRUCT PSYCHOLOGY ..........227

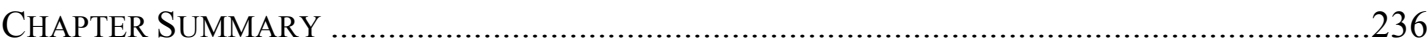

CHAPTER 8 - HERMENEUTIC CONSTRUCTIVISM: IN APPLICATION....................238

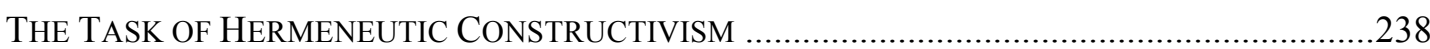

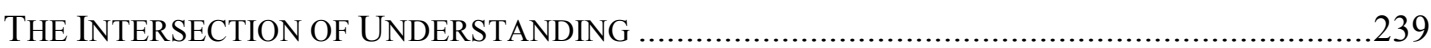

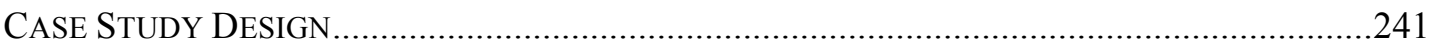

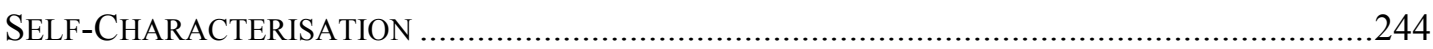

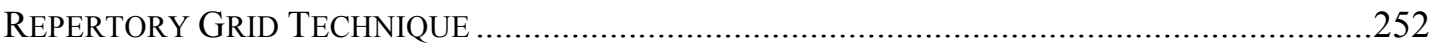

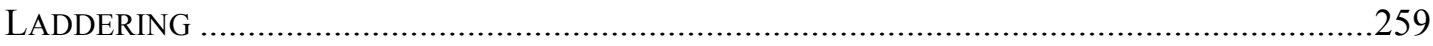

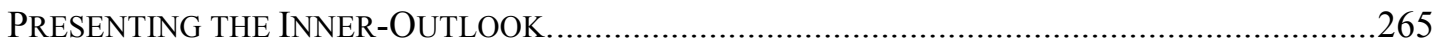

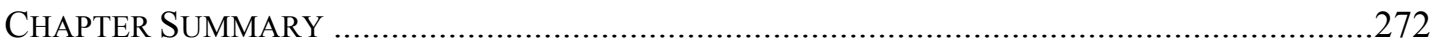

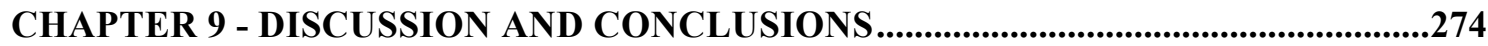

LOOKING BACK IN ORDER TO REDRESS THE FUTURE. .........................................................274

IMPLICATIONS AND PRODUCTIVE POSSIBILITIES OF HERMENEUTIC CONSTRUCTIVISM ......289

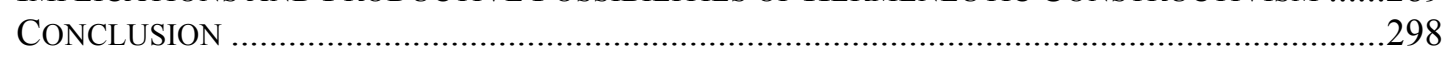

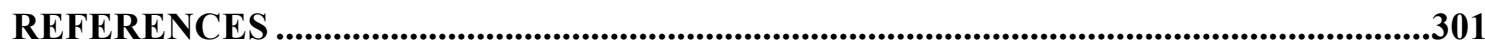




\section{LIST OF FIGURES}

FIGURE 1.0: AN EXAMPLE OF A DIALOGICAL EXPERIENCE PROFILE 254

FIGURE 2.0: WHEN TO ASK 'WHAT IS REQUIRED FOR THAT TO HAPPEN 258

FIGURE 3.0: SELECTING AN APPROPRIATE PREJUDICE FOR LADDERING 263 



\section{CHAPTER 1}

\section{SIGNIFICANCE AND ORGANISATION}

It is the prospect of representing human experience with phenomena that makes the adoption of qualitative research practices compelling. Undeniably, researchers seek to provide qualitative representations that maintain an intimacy with their subjects' realities. However, within the historical backdrop of qualitative research lies a period of thought commonly referred to as the Crisis of Representation. At the core of this crisis is a challenge that problematises the assumption of a direct link between language and experience. Arguably this view of the relationship between language and experience remains pervasive within qualitative research today. This is the assumption that language unambiguously points toward or designates a person's experience, an assumption that closes down the possibility that words carry meaning that goes well beyond simply what is said. The capacity of language to disclose the world differently for each person, in unique ways, means that the qualitative researcher subscribing to such a designative account of language can no longer be considered to have directly captured the lived experience of his or her subject. It is therefore the view of this thesis that the representations of experience presented in qualitative research are in fact views constructed by the researcher and not necessarily those of the real people or the real lives that are encountered in research.

What is it then about qualitative research that makes it qualitative? For this thesis it is the opportunity to make apparent a subject's qualia or the qualities of experience as perceived or understood by a person. The following passage from Dennett (1988) captures what is meant by the notion of qualia for this thesis.

Qualia is an unfamiliar term for something that could not be more familiar to each of us; the way things seem to us. As is often the case with philosophical jargon, it is easier to give examples than a definition of the term. Look at a glass of milk at sunset; the way it looks to you - the particular, personal, subjective visual quality of the glass of milk is the quale of your visual experience at the moment. The way the milk tastes to you then is another gustatory quale, and how it makes sounds to you as you swallow is an auditory quale; these various "properties of conscious experience" are prime examples of qualia. Nothing, it seems, could you know more intimately than your own qualia. (p. 11)

Qualia are therefore the qualitative character of experience. They thus represent precisely those idiographic elements that should be the focal point of qualitative research. This passage 
from Dennet emphasises the way that qualia are inherently peculiar to each individual person. In order that this inherently peculiar nature of a person's qualia can become the focus of qualitative research, the qualia itself must first be able to come to language. The fact that each of us is able to be articulate about the quale of milk that Dennett draws our attention toward means that qualia are indeed within the reach of language. On this assumed link between qualia and language this thesis accepts as a beginning point the words of Wittgenstein's (1922/1999) final proposition of his work Tractatus Logico-Philosophicus which contends, "Wovon man nicht sprechen kann, darüber muß man schweigen"- "What we cannot speak about we must pass over in silence" (p. 108). This thesis takes the view that things of which nothing can be said cannot be understood and are therefore of little concern to this thesis and the qualitative researcher.

Returning to the issues concerning language, a person's qualia represent a considerably high level of abstraction with which to contemplate that person. However, it is this level of abstraction that the thesis suggests to be beyond the reach of contemporary qualitative research. That is, to assume the unproblematic link between language and the qualities of human experience that is necessitated by the current approaches to qualitative researcher, ultimately robs language of its expressive capacities and therefore robs the participant of having his or her real and highly nuanced qualities of experience represented by qualitative research. In other words, to overlook the capacity of language to disclose the world in a manner that is different for each of us and instead to subscribe to a designative account of language, where words simply point to things in the world, closes down the idiographic potentialities of understanding the world and of being human. Without adopting a view of language that recognises that words carry meaning that go well beyond simply what is said, the inherently idiosyncratic nature quale cannot be apprehended in all its minutia. Therefore, any account of a person's qualia founded upon a designative account of language cannot be considered to genuinely represent the subject matter of focus - the qualities of an individual person's experience. This thesis therefore aims to develop a new theoretical approach that redresses these challenges to representation by emphasising the centrality of the dialogic in understanding. Specifically, it delivers a more theoretically robust approach for understanding a person's idiographic qualia and therefore provides a deeper representation of the person for qualitative research.

At the core of the challenge to representation in contemporary qualitative research is an opposition to the pervasive adherence to thematic representation. Thematic representations are unavoidably beholden to the naïve or designative view of language and meaning noted above. These assumptions about language are perpetuated by a necessity for 
the qualitative researcher to 'make-meaning' after the dialogical opportunity itself has passed. In order that the researcher can come to an understanding of the participants' experience, he or she must assume that the words of the participant carry unambiguous meaning that can somehow be directly accessed. It follows therefore, that in the development of a theme, the researcher makes meaning of the experiences of each of the participants. The researcher then searches for commonality across the meanings that he or she has made of the participant's experiences and clusters these meanings together to produce a theme. The theme that is produced is considered to be representative of the experiences of the people involved in the qualitative research. However, this thesis suggests that the understanding that is being produced by these practices is less of a representation of the individual participants, and more a representation of the researcher. It is argued that at the foundation of these representational challenges is a subscription to naïve assumptions about language and meaning. Therefore, it is ultimately the perpetuation of these theoretical assumptions about the nature of language that are responsible for the dearth of focused and deep understandings of human qualia and the individual(s) that have them. As well as this, the failure of qualitative research to capitalise on the dialogical opportunity that the researcher has with the participant negates any claim by current qualitative research to represent an individual's qualia in a meaningful and productive way. This thesis can therefore be seen as a response to the following question that emerges from these challenges.

Is it possible that qualitative research has contributed less than would seem attainable to the study of individuals because we as qualitative researchers have been working on the basis of a number of assumptions about people, which we have not examined very carefully, and have also adopted a number of assumptions about the nature of science which are less than adequate for the subject matter we have chosen? ${ }^{1}$

Thus the aim of this thesis is to develop an approach to qualitative research that provides a deeper understanding of the highly nuanced qualia of an individual, one that 'transcends the obvious'. More specifically, the thesis develops a new theoretical position that informs qualitative research. It overcomes the representational challenges identified and provides an opportunity for a deeper consideration of the experiences of the individuals involved in qualitative research.

\footnotetext{
${ }^{1}$ Parallels can be drawn here with Mair (1970) 'Experimenting with individuals', where he calls for us to think of a more adequate alternative to the study of human life than the 'physical sciences' model that dominates psychological investigation. He believes this model does not take adequate account of important features of the subject matter of focus - the person.
} 


\section{Theoretical Framework and Direction}

In order to appreciate the challenge being levelled at qualitative research - that it represents less than would seem attainable despite its focused study of individuals - requires a comprehensive theoretical analysis of qualia and the way they can be represented in qualitative research. Inevitably, this will touch on notions of being and reality; these are profoundly complex phenomena. By way of introduction, this section offers a general overview of the ideas to be developed by this thesis.

The thesis begins with a synopsis of current knowledge on issues of representation within qualitative research. Despite the exponential growth in the area of qualitative research, there is very little accord amongst qualitative researchers about the implications of a series of under-examined assumptions that concern the representation of a real. Indeed, what representation $i s$, remains an open issue. The debate is exemplified by significantly divergent views about representation itself and its importance within qualitative research. This is often termed the Criteria Debate and is discussed in Chapter Two.

In the context of qualitative research the idiographic or nuanced aspects of one's qualia arguably do not lend themselves to being represented by the processes required of traditional thematic representations. The search for superficial generality between the qualia of one person and the equally nuanced qualia of other people, represents a disregard for the depth of understanding that is considered possible. In fact, it is suggested that by focusing in on the peculiar rather than the moving out to the superficial and the general that is typical of much qualitative research, a greater depth of understanding can be achieved. Therefore, this thesis is located within the theoretical domain of idiographic science, where these within person elements of being are the primary focus of consideration and overcome any obligation toward generality.

A theoretical model of the person that is sympathetic to the idiographic perspective while consistent with the level of data abstraction with which this thesis envisages as possible is required. Embodying in principle this idiographic position and offering a theoretical consideration of the within person elements of being is George Kelly's The Psychology of Personal Constructs. Kelly (1955) suggests that individuals develop for themselves a series of constructs by way of which they come to understand the world around them. For Kelly the construct is a primary structure of being and as such is consistent with the particularly high level of abstraction that this thesis considers to be most productive. Thus, from the purview of the Psychology of Personal Constructs, the illumination of a person's constructs represents a deep understanding of that person. Despite the apparent synergies between this position and 
the aims of this thesis, the insufficient way in which Kelly (1955) dealt with language challenges the theoretical reflexivity of his position and its overall theoretical tenability as a framework for the qualitative research approach that is being developed by this thesis. These issues are addressed in Chapter Three and Chapter Four.

On the basis of the unsophisticated way in which Kelly dealt with language, and the subsequent implications for the representation of a real, the thesis also draws on the work of Hans-Georg Gadamer. His elucidation of language as indissoluble from being - found in his major work Truth and Method - demonstrates that language is the medium by way of which a person comes to understand the world. So much is this the case for Gadamer that nothing can be understood independent of language. Gadamer's philosophical hermeneutics - used pragmatically here - provides a position from which to re-envision several of Kelly's key philosophical principles that will be identified as theoretically weak. Termed Hermeneutic Constructivism, the theoretical outcome of this re-envisioning of Personal Construct Psychology arguably represents an opportunity to elucidate the experience or qualia of the individual people involved in qualitative research at a particularly high level of abstraction. In so doing, Hermeneutic Constructivism will therefore provide a deeper; more sophisticated and nuanced understanding of the experiences of the real people encountered in qualitative research.

\section{Overview of the Research}

The thesis is divided into two sections. The first section consists of Chapters Two to Five and provides a critique of the issues that surround representation within qualitative research as well as delineating the potentialities that an idiographic approach might hold for overcoming these challenges to representation. It is suggested that pursuing the idiographic approach to understanding human experience will provide a deeper illumination of the real people encountered through qualitative research. To begin, Chapter Two, Representation in Qualitative Research, outlines the assumptions about language and what it is to be a person that operate to limit the claim to representation that can be made by contemporary qualitative research. A review of the Criteria Debate emphasises the ongoing lack of consistency and even concern within qualitative research for the representation of the real people and real lives encountered. This chapter culminates in a suggestion that making the individual person the focus of consideration may provide an avenue for addressing these representational challenges, a position embodied by the Idiographic approach. Chapter Three, The Idiographic Perspective, emphasises that the idiographic account is not merely another perspective from which to envision the human subject, instead it represents the position that embodies the nature of being human itself. Embodying - in principle - an idiographic 
account of the person is George Kelly's Personal Construct Psychology. His work not only embodies these idiographic principles but also concerns itself with a level of abstraction of the person that is envisaged as integral for Hermeneutic Constructivism. Therefore this chapter provides a review of Kelly's position. However, a close reading of Kelly's work in fact highlights an inadequate consideration of the role that language has in the process of construing. If indeed the principles that are inherent within Kelly's work are to be a productive foundation for the aims of this thesis, then his theoretical unpacking of the role of language in understanding stands in need of refinement. Thus, Chapter Four, Kelly's Nä̈ve View of Language, critically examines Personal Construct Psychology drawing attention to the key theoretical implications of Kelly's inadequate account of language. In fact this critique culminates in a view that the absence of an adequate account of language within Personal Construct Psychology challenges the theoretical tenability of Kelly's overall position. Suggesting therefore that Personal Construct Psychology is open to development in the direction of language Chapter Five, Language as The Missing Medium, undertakes a consideration of Hans-Georg Gadamer's Hermeneutic Phenomenology. Gadamer's work situates language as the essential medium of being human and in so doing provides a theoretical foundation for re-envisioning Personal Construct Psychology and subsequently the development of Kelly's position in the direction of language. The outcome of this reenvisioning is the theoretical position termed Hermeneutic Constructivism and is the focus of the second section of the thesis.

The second section consists of Chapters Six to Nine which set-down the Hermeneutic Constructivist position as well as the way a researcher would conduct qualitative research as informed by the theoretical tenets of this new approach. This section culminates in a consideration of the way in which the Hermeneutic Constructivist approach is able to address the representational challenges identified within qualitative research. This section also outlines how the Hermeneutic Constructivist approach can be seen to progress many of theoretical principles of Personal Construct Psychology in the direction of language and in so doing provide a tenable approach to the potentialities of an idiographic understanding of the person. The final section of this chapter provides a consideration of the productive possibilities of the Hermeneutic Constructivist position to weigh into several ongoing areas of debate and to subsequently provide alternate avenues to pursue. Beginning this section is Chapter Six, The Idiographic within a Languaged World, which by adopting the model for elaborating a theory as outlined by Kelly (1955) advances the Fundamental Postulate and elaborative corollaries of Hermeneutic Constructivism. Given the complex and at times abstract nature of the theoretical assumptions that underpin the Hermeneutic Constructivist approach, Chapter Seven, Hermeneutic Constructivism in Elaboration, provides a closer 
examination and discussion of these more abstract notions. Recognising that it is difficult to assess the degree to which Hermeneutic Constructivism achieves the goals that have been set for it by this thesis outside of its application in qualitative research, this chapter also outlines the way in which the Hermeneutic Constructivist position is consistent with the criteria of good theory that Kelly (1955) proposes. In addition, the chapter takes the same framework that was used to evaluate the theoretical tenability of Personal Construct Psychology as a touchstone for evaluating the developments made by Hermeneutic Constructivism in terms of a re-envisioning of Personal Construct Psychology in the direction of language. Chapter Eight, Hermeneutic Constructivism in Application, outlines a method for conducting qualitative research that is informed by the tenets of Hermeneutic Constructivism. The core focus of this chapter is the Dialogical Experience Profile and its subsidiary approaches which are proposed as a vehicle for accessing, elaborating and presenting the high level abstractions with which Hermeneutic Constructivism concerns itself. Finally Chapter Nine, Hermeneutic Constructivism Discussion and Conclusions outlines the way in which the Hermeneutic Constructivist position can be considered to have addressed the aims of the thesis by providing an approach that is able to articulate a deeper understanding of the real people encountered in qualitative research. A discussion of how the Hermeneutic Constructivist approach can be seen to overcome the assumptions about language, meaning, and people in general that lead to misrepresentation in contemporary approaches to qualitative research is provided. In addition, a consideration of the way that research informed by Hermeneutic Constructivism can be considered to be compatible with the principles of validity, reliability and generalisability is also undertaken. The chapter and thesis conclude with a discussion about the productive possibilities that a Hermeneutic Constructivist perspective might offer some ongoing debates; including considerations of the Self and the theoretical bridge between the personal and the social modes of being human. 


\section{CHAPTER 2}

\section{REPRESENTATION IN QUALITATIVE RESEARCH}

The previous chapter outlined the structure of the thesis. This chapter will consider the way a series of uncritically accepted assumptions operate to challenge the claim qualitative researchers can make to representing the real people and real lives of participants. In order to achieve this aim, key aspects of the qualitative research process will be critiqued to highlight the pervasive adherence to a naïve assumption about language and a perceived transparency of meaning, both of which have implications for representation. An examination of the Criteria Debate underscores further the widespread disagreement about representation within qualitative research. This chapter culminates by establishing that the focused consideration of the individual person is a productive avenue to pursue in addressing these challenges to representation.

\section{Representing 'A Real' in Qualitative Research}

One of the unique features of qualitative research is the intimate entanglement of issues concerning representation and epistemology. This entanglement is highlighted by a tension between what is to be represented and the degree to which the representation is an accurate depiction. Participants in qualitative research enjoy an epistemic privilege by virtue of the fact that it is a dialogue about their experiences that is of interest to the qualitative endeavour and no one can know these experiences as intimately as they do. Researchers, on the other hand, have access to literature and theoretical models through which to frame a participant's experience within a wider context. For Gergen and Gergen (2000) this produces a state whereby the "division between researcher and subject is blurred, and control over representation is increasingly shared" (p. 1035). However, this effort at democratising the research endeavour opens up a myriad of ethical and methodological challenges (KarnieliMiller, Strier \& Pessach, 2009), that ultimately lead one to question: Whose representation is being represented?

It would not be unreasonable to expect one possible response to this question to be framed as a further question: Why is the untangling of such concerns of any interest to qualitative research more broadly? That is, why should qualitative researchers be concerned 
with the degree that the representations produced are genuinely representative of the real people and real lives they purport to represent? Why should qualitative research be aligned with the tents of a realism? This thesis suggests that qualitative researchers need to situate themselves as operating within the spectrum of realism in order to lay claim to any sense of legitimacy for their work or for having provided a productive approach for unpacking the vicissitudes of being human. This is so much the case that to adopt a contrary theoretical position to a realism gives up on the possibility that qualitative research is capable of developing genuine and legitimate understanding of the subject matter of focus - individual human experience. ${ }^{1}$

What is ultimately at stake here for qualitative research then, is the claim that can be made for genuinely representing r real $^{2}$ This concern is embodied by what Denzin and Lincoln (2005) refer to as the "crisis of representation" (p. 18). ${ }^{3}$ At its core this crisis challenges the extent to which the qualitative research endeavour can be considered to develop a representation that is faithfully and fairly commensurate with the experiences of the human subject. Although qualitative researchers have traditionally sought solace in the more intimate and therefore supposedly more accurate representations of the lives of those studied, Sandelowski (2006) suggested this belief to be "naïve, at best and hubris, at worst" (p. 10). Recognising that human experience is not simply discovered, rather constructed by researchers and their interpretations, Sandelowski (2006) goes on to allude to the risks of perpetuating what she terms a ventriloquism ${ }^{4}$ where, "instead of giving voice to the voiceless, qualitative researchers have too often engaged in controlling the voices of the voiceless, maintaining their voicelessness" (p. 10). In a critique of qualitative research, presented in The

\footnotetext{
${ }^{1}$ In taking such a position this thesis shares Bourdieu's (1999) view and recognises that even if one objects to the possibility of a real there are serious consequences for a position that abandons its search.

${ }^{2}$ It is important to make clear the use of the phrase 'a real'. At the core of the theoretical position that is ultimately developed is a rejection of the possibility of a totality of understanding. In other words, this thesis suggests that it is not possible to achieve a perfection of understanding. It is for this reason that the use of the term 'the real' has been avoided. Instead, the use of ' $a$ real' indicates that there are infinite possibilities of understanding that can be developed by a person and yet are at the same time equally legitimate. Thus, when this thesis talks about the representation of 'a real', it is concerned with the degree to which a representation is confluent with the qualia of the person being represented. In contrast, a confluence with a supposedly universal or given real as 'the real' is of little concern given the theoretical impossibility of establishing such a position.

${ }^{3}$ Marcus and Fischer (1986) are often credited as the first to bring to the attention of the wider qualitative research community the notion of a crisis of representation within qualitative research. However, many of the tenets of their argument can be seen within the pages of the earlier work of Rorty (1979/2009) Philosophy and the Mirror of Nature.

${ }^{4}$ Fine (1994) makes reference to the notion of ventriloquy and elaborates it as a technique for representing the human subjects. At the core of ventriloquy is a desire to represent a person as a subject while treating them as objects. For further reading on the topic of ventriloquy see Fine (1994) 'Dis-stance and other stances: negotiations of power inside feminist research' in Gitlin (Ed.) Power and Method: Political Activism and Educational Research.
} 
Sage Handbook of Qualitative Research - often considered the definitive text in the area Ho, Ho and $\mathrm{Ng}$ (2007) emphasise what they believe to be the disregard for rigour, scholarship and ultimately quality that is evident within qualitative research.

Qualitative research appears to thrive on perennial crisis, of legitimization, of praxis, and of representation. There is an abundance of theorists, but a dearth of practitioners; a preponderance of assertions, but little evidence or rational argument in support of them (p. 382).

There can be little doubt that the ultimate endeavour of qualitative research is to represent that toward which the focus of consideration is directed - to represent real people and real lives. However, this thesis takes the view that a series of insufficiently examined theoretical assumptions that underpin the 'crisis of representation' within qualitative research, operate to undermine the possibility of qualitative research to do just that - to represent this real.

At the centre of this contention is a belief that the pervasive subscription to naïve assumptions about language and the transparency of meaning carried by language - arguably necessitated by a failure to capitalise on the dialectic opportunity of understanding - operates to close down the possibility of qualitative research to genuinely represent real lives and real events. This opens qualitative research to the challenge of providing the scientific community an inadequate, irresponsible and misleading misrepresentation of the participant's experiences. Here one is drawn back to the initial question that emerges from these challenges to the current practices of qualitative research

Is it possible that qualitative research has contributed less than would seem attainable to the study of individuals because we as qualitative researchers have been working on the basis of a number of assumptions about people, which we have not examined very carefully, and have also adopted a number of assumptions about the nature of science which are less than adequate for the subject matter we have chosen?

As suggested previously the assumption at the core of this challenge concerns the naïve account of language and meaning that is necessitated by the closing down of the dialogical opportunity with the participant, a practice that is pervasive within contemporary qualitative research practice. However, prior to exploring the influence of this assumption upon several key moments that are common to the practice of qualitative research, a closer examination of what is meant by these naïve assumptions about language and meaning is necessary. 


\section{The Naïve Approach to Language and Meaning}

A naïve approach to language assumes that language is merely a straightforward or unambiguous tool that functions to represent things in the world. Such an account overlooks the fundamental capacity of language to disclose the world and, instead, epitomises what Taylor (1985) describes as a designative account of language. Taylor's (1985) primary concern is specifically with theories of meaning and by extension theories about the fundamental nature of language. As a function of this he offered two accounts of language: the 'expressive' and the 'designative'. These provide a useful model for unpacking what is meant by naïve assumptions about language here.

\section{The Expressive Account of Language}

Although it is the designative account that this discussion is most concerned with clarifying here, a consideration of expressive accounts provides a valuable touchstone for emphasising those aspects of language overlooked by designative accounts. For Taylor (1985) expressive accounts maintain "some of the mystery surrounding language" (p. 221) and account for language as constitutive of thought and subsequently as disclosive of the world. In terms of qualitative research, expressive accounts maintain that meaning cannot be separated from the language that conditions it, because meaning is only manifest in it. Thus, epitomising the expressivist account, this notion recognises that although it is a public language that we share, the carriage of meaning in language cannot be known outside of the expression of this meaning in dialogue. This is emphasised by Taylor (1985) in the following passage

The meaning of an expression cannot be explained by its being related to something else, but only by another expression. Consequently, the method of isolating terms and tracing correlations cannot work for expressive meaning...expression is the power of a subject; and expressions manifest things, and hence essentially refer us to subjects for whom these things can be manifest. And as I said above, what expression manifests can only be made manifest in expression, so that expressive meaning cannot be accounted for independently of expression. (p. 221)

Expressive accounts therefore depict language as being more than simply what is said, it is in Taylor's (1985) words, "always more than we can encompass; it is in a sense inexhaustible" (p. 231). Therefore an expressive view accounts for language as disclosing of a view of the world that can be made manifest only in expression. In gathering all of this together Taylor 
(1985) captures the core tenets of the expressive account of language in the following passage.

The expressive conception gives a view of language as a range of activities in which we express/realize a certain way of being in the world....it is not just the reflective awareness by which we recognize things as -, and describe our surroundings; but also that by which we come to have the properly human emotions, and constitute our human relations...it is more like a medium in which we are plunged, and which we cannot fully plumb. (pp. 234-235)

In order to illuminate more adequately the nature of an expressive account of language, a consideration of what it is not is valuable. For this reason a return to a discussion of Taylor's (1985) designative account of language is worthwhile.

\section{The Designative Account of Language}

Designative theories of language make designation fundamental and therefore make meaning a quality that is decidedly unpuzzling and unmysterious. At the core of a designative account is a naïve assumption that language as utterance and as inscription makes sense because at some level they represent and stand-for something in the world or re-present something to the mind (Lawn, 2004). To say that language represents something here is to suggest that it points to or designates something. Here words are simply ciphers or signs for things and those words are meaningful as a function of their association with the thing for which they stand proxy. This unambiguous account of language means that for designative approaches words 'stand for' or 'signify' the things they represent. The core principle that perpetuates the acceptance of designative approaches to language is the promise of a theory of language that makes it unmysterious and unpuzzling so that it can be identified objectively and fit within the canon of natural science.

Scientific thought needs to be objective and therefore it must give an account of the universe that is independent of subject related properties. Taylor (1985) describes these as "the properties that things have in the experience of subjects, and which would not exist if subjects of experience did not exist" (p. 221). It is clear therefore that an expressive account of language cannot avoid subject-related properties. Given that what expression manifests can only be made manifest in expression itself, expressive meaning cannot therefore be accessed independently of expression and therefore cannot meet the requirements of scientific thought. However, it is this promise of freedom from a subject-related account of language that propels designative accounts of language. So much so, that to use the words of Taylor (1985), a designative account of language "must be perfectly transparent; it cannot itself be 
the locus of mystery, that is, of anything which might be irreducible to objectivity. The meaning of words can only consist in the ideas (or things) they designate" (p. 226). Thus, here words attach to the thing they designate. Therefore for designative theorists, each of us is able to capture phenomena by appealing to the way that words mean what they designate and can therefore provide an accurate representation of the way things are.

One of the central issues that divide expressive and designative accounts of language is the role of language within thought. One of the principal concerns of British empiricist thought found in the work of Hobbes and Locke was to establish a picture of the empirical world that was grounded in the foundation offered by a clear unequivocal definition of words (Taylor, 1985). In order to achieve this, language needed to be demystified showing it to be merely an instrument of thought. For Taylor (1985) "one of the stronger motives for making it so basic was the desire to overcome projection, and what we later call 'anthropomorphism', that promiscuous mixing of our own intuitions of meaning, relevance, importance with objective reality" (p. 249). Thus, for language to be an instrument capable of building a representation of the universe it must be clear what aspects of the world words attach to, what they connect with or designate, and this connection must be completely within our control.

In exploring this link between thought and language further, if language is simply an instrument of thought, that is, it signifies or designates it, then it is palpable that thought must occur prior to language itself. On this Lawn (2004) poses the following question, "if language is merely a mnemonic aid to thinking (language somehow makes complex thought more manageable according to Hobbes), what then is thought?" (p. 8). Designative accounts would have us believe that thought is wordless until converted into sound and then into meaning that is accessible to the mind. In response, Lawn (2004) seeks to undermine the tenability of the designative position.

Why, contra this view, is it the case that cognitive development goes hand in hand with development of linguistic ability? Surely a child only acquires sophistication in its thinking as it becomes more adept in the use of language? The belief that ideas and thoughts precede language (logically and chronologically) is surely untenable, as we will see when we turn to nondesignative accounts of language. (p. 8)

An adherence to a designative account of language, despite the untenable nature of the position, clearly signifies a focus that is less about the nature of language - as a series of ciphers or token - but as a way into a scientific study of the individual person's inner mental life. So much is this the case that Lawn (2004) suggests that in designative accounts of 
language "questions about the actuality of language are quickly overshadowed or forgotten. The diverse and extensive nature of language is overlooked and a limited concentration upon the dubious belief that language discloses states of mind neglects the phenomena of language" (p. 9). It is this neglect of language's extensive and expressive nature and the subsequent subscription to a naïve or designative account of language that permeates contemporary approaches to qualitative research and ultimately underpins the challenge being levelled by this thesis. So much so, that from this point onwards, any reference to naïve or transparent theoretical assumptions within this thesis, relates to the account of language and meaning outlined here as a designative account.

Within contemporary approaches to qualitative research the representations of the real people and the real lives encountered are contingent, at some level, upon a subscription to this naïve or designative account of language. Recall that in such an account the meaning of words and sentences is not contingent upon their expression. Instead, words carry supposedly unambiguous meaning that can be clearly and readily accessed by another person. The necessity for a qualitative researcher to make meaning of the words of a participant after the dialogical opportunity with that person has passed - a practice required of contemporary approaches to qualitative research - certainly assumes at some level the possibility of a designative account of language. Here the words uttered by the participant must point toward meaning that is transparent or that the researcher can simply apprehend and then set down as a supposedly legitimate representation of the real people and real lives encountered. However, given that designative approaches to language have been emphasised by Lawn (2004) as dubious and in fact untenable on the basis that they overlook the essentially expressive and disclosing characteristics of language, one can legitimately ask 'who or what is in fact being represented by qualitative research that subscribes to such a position?'

In order to highlight the pervasive subscription to these designative accounts of language and the practices that necessitate a subscription to this account of language, a close examination of several key moments that are common to the practice of qualitative research is valuable. Considered here in isolation, the key moments of qualitative research: (i) the research interview, ${ }^{5}$ (ii) the production of meaning, and (iii) the research presentation, will be illuminated in order to underscore the way that naïve assumptions about language pervade qualitative research. As well, this discussion will emphasise the way that these common

\footnotetext{
${ }^{5}$ It must be said that the research interview is not the sole methodological enterprise of the qualitative research paradigm. Despite this, the research interview is the most widely used modality for garnering research data among contemporary qualitative approaches (Gubrium \& Holstein, 2002; King \& Horricks, 2010). It is for this reason that the model of the research interview is worthy of investigation in more depth enabling the subsequent unravelling of some of the problematic assumptions embodied by its use.
} 
practices lead to a progressive movement away from the representation of the real people and real live encountered.

\section{The Research Interview}

Listening to and recording an individual's experience of, and with, worldly phenomena is the critical challenge for the qualitative researcher; not withstanding the translation of what is "heard" ${ }^{6}$ into representation. The research interview ${ }^{7}$ represents the totality of the opportunity available to the qualitative researcher to 'catch a glimpse' of what being human is for a particular participant. In other words, the research interview is the embodiment of that dialectic venture from which the understanding that is produced ultimately originates. This thesis takes the view that the perception of the research interview as being inherently benign and genuinely dialogical in nature is an assumption that raises further the spectre of doubt concerning the representation of a real by conventional approaches to qualitative research.

Some of the most imaginative and rigorous considerations of the theoretical and ethical conundrums implicit within the research interview can be found within Bourdieu's (1999) work. ${ }^{8}$ Providing a most creative and confronting critique, Bourdieu (1999) focusing upon the plight of the downtrodden in France - classified the research interview in terms of a 'trap' that leads the uncritical researcher toward the position of "false, collusive objectification" (p. 216). ${ }^{9}$ The following passage from Yanos and Hopper (2008) provides an authoritative elaboration of Bourdieu's (1999) position.

False, because it is unfaithful to the particularities of the life under scrutiny; collusive, because the interviewer unwittingly goes along with the artifice; and objectifying because a readymade account has been substituted for the lived, unfinished angularities of the reflective subject. The net result is that respondents effectively elude the interview's incentives, opportunities and

\footnotetext{
${ }^{6}$ It is important to make clear that to hear does not imply that one understands.

${ }^{7}$ The deferment to the term dialogue over research interview, which has gained widespread appeal amongst qualitative researchers, is in fact a misnomer given that a dialogue involves a mutual interest, while ultimately here the interest sits most squarely with the interviewer (Brinkman \& Kvale, 2005; Gubrium \& Holstein, 2002; Kvale, 1996, 2006; Kvale \& Brinkmann, 2009; Scheurich, 1995, 1997). ${ }^{8}$ The most significant of Bourdieu's works with which this thesis concerns itself here is: Bourdieu (1999) The weight of the world: Social suffering in contemporary society.

${ }^{9}$ Recognising the quality of the research interview as central to the representation that is produced, Bourdieu (1999) recommended that researchers develop a keen sense of 'discernment' and be aware of being caught in the 'trap' of 'illusory insights' and 'counterfeit visions'. He goes on to suggest, that if one suspects that he or she has succumbed to the trap then the interview should be classified as slipping into 'false, collusive objectification' and be dropped from analysis and therefore the final research presentation.
} 
instructions, all of which have been formally designed to secure some

modicum of truth peculiar to the singularities of this respondent. (p. 230)

Bourdieu (1999) conceptualises the uncritical research interview as a contrived attempt to coerce the interviewee into agreeing with a series of preordained intentions of the interviewer. According to Yanos and Hopper (2008), Bourdieu (1999) suggested that agreement by a participant with these pre-established notions of meaning is deceptively pleasing for the researcher. Bourdieu (1999) goes on to argue that the research interview provides no more than an unsophisticated account of the respondent. By subscribing to little more than the search for agreement with the researchers preordained account - therefore closing down the possibilities of the dialectic - the researcher that Bourdieu (1999) has in mind cannot evade the challenge of providing merely naïve accounts of the participant.

In contrast to research interviews that reach toward this kind of contrived and naïve effort of self-fulfilment, Bourdieu (1999) conceptualises the ideal interview in terms of a 'spiritual' exercise where researcher and respondent work in a "simultaneously painful and gratifying effort" (p. 616), it is a dialogue through which they explore the issues that one may find difficult to elaborate. Concerned also with the ideal research interview, Brinkmann (2007) in an article titled 'Could interviews be epistemic? An alternative to qualitative opinion polling', begins with the provocation "Could we, by means of qualitative research interviews, gain knowledge?" (p. 1116) Anticipating the irritation of those within the qualitative domain, Brinkmann clarifies his position regarding the type of knowledge with which he is concerned. He suggests that conventional approaches to qualitative research do in fact yield knowledge, but knowledge in the form of doxa or opinion as opposed to knowledge in the sense of episteme. ${ }^{10}$ The following passage from Brinkmann (2007) is explanatory of this position and points toward a need for reconceptualising the way in which research interviews are conducted by advocating a move in the direction of a genuine dialogue. ${ }^{11}$

\footnotetext{
${ }^{10}$ This thesis suggests that the outcomes of qualitative research produce little more than opinion or doxa. In fact this thesis will later emphasise that the unsustainable nature of some core theoretical aspects of Personal Construct Psychology operates to underscore the doxastic qualities of the construct itself.

${ }^{11}$ Exploring the potentialities of other approaches to the research interview Brinkmann (2007) and Kvale (1996, 1999, 2003, 2006) are bemused by the widespread neglect of the psychological perspective within discussions concerning the research interview. Given the clear influence of the client-therapist relation and the framing of the interviewer-respondent relationship within qualitative research Ferudi (2004) and Feltham (2010) suggests the therapeutic interview sits comfortably within qualitative research. These authors go on to suggest that such an approach is sympathetic to the therapy culture that underpins what Atkinson and Silverman (1997) term the current "interview society" (p. 314) within which qualitative researchers operate.
} 
It is about the interview respondents' experiences and opinions, which when viewed through the lenses of classical philosophy - rarely constitute knowledge in the sense of episteme, that is, knowledge that has been found to be valid through conversational and dialectical questioning... in simple words: By probing their respondents' experiences and opinions (the doxa), interview researchers are often engaged in what seems like a time-consuming kind of opinion polling for which qualitative instruments such as questionnaires often appear to be much more efficient. If we should really take advantage of the knowledge-producing potentials inherent in human conversations, such as research interviews, ought we not frame the interview situation differently? (p. 1117)

Brinkmann (2007) acknowledges that his work is not an attempt to provide a grand critique or catalogue of contemporary interview forms and that his intentions are perhaps utopian. Despite this, and with inspiration from the Socratic method, his conviction is toward interviews that operate epistemically to produce knowledge and not simply opinion in the way that is consistent with contemporary qualitative interviewing practice. ${ }^{12}$ In other words, Brinkmann (2007) recognises the productive possibilities of dialogue where one is free to challenge and question in a genuine movement of understanding. Here Brinkmann (2007) as too this thesis - recognises the potentialities of understanding that are possible within the dialogical encounter itself. In fact by advancing the opportunity that is present in the dialectic with a research participant, the subsequent necessity for naïve meaning making on the part of the researcher can be overcome. Indeed for Ferudi (2004), advancing the dialectic within qualitative research will go some way toward overcoming the need for researchers to impose labels upon the inherently more complex emotions and experiences of their participants. This is key because the commodification of a participant's most inner emotions by a researcher within contemporary research interviews itself underscores a range of ethical issues of representation that are inherent within the research interview (Ferudi, 2004).

\footnotetext{
${ }^{12}$ It is for this reason that Brinkmann (2007) suggests that qualitative research approaches should consider more seriously the way in which interviews are conducted. He suggests a turn toward an epistemic interview, an interview that leads to knowledge over and above the merely doxastic interviews that are common in conventional qualitative research and that garner merely opinions and beliefs. Following the lead of Socratic interviewing, Brinkmann (2007) suggests a more 'challenging' approach to interviewing that encourages disagreement, questioning and challenging what an interviewee says. A considered look at further epistemic interviewing will be undertaken later in this thesis. For a sound consideration of the principles and practices of Socratic Interviewing see: Dinkins (2005) 'Shared inquiry: Socratic-hermeneutic interpre-viewing'.
} 
For many authors within contemporary qualitative research, the ethical implications of misrepresentation as a direct result of the interview process itself are merely papered-over (Brinkmann, 2004, 2007; Brinkmann \& Kvale, 2005; Burman, 1997; Kvale, 1996, 2003, 2004, 2006). Brinkmann and Kvale (2005) make a useful distinction between micro and macro ethical concerns within the research interview. Micro ethical concerns focus upon the relationships within the interview, while macro ethical issues mark a consideration of the relations of the representations produced to the wider society and culture at large. Micro ethical concerns within the interview shape the representation that is produced and thus lead toward the broader or macro ethical implications that Brinkmann and Kvale (2005) suggest.

At the micro ethical perspective, Burman (1997) suggests that the commonly held view that the research interview is ethically superior because it is free from manipulation and instrumentality is a fantasy. Sharing this view, Kvale (2006) suggests qualitative researchers often succumb to an uncritical acceptance of what he has termed the "qualitative progressivity myth" (p. 481). Here a researcher believes that the research interview is implicitly 'above all' good and emancipating. However, Brinkmann and Kvale (2005) suggest that the research interview carries an inherent - and uncritically accepted - asymmetrical, instrumental and manipulative dialogue that is always "following a more or less hidden agenda" (p. 164). Such a position is supported through the work of several other scholars (Burman, 1997; Glesne \& Peshkin, 1992; Mauthner, Birch, Jessop \& Miller, 2002). ${ }^{13}$ Watson (2006) for example, embodying the collective argument, suggests that "the idea of a 'real self' permeates much of the work associated with the 'traditional' qualitative interview leading to conceptualizations of the respondent as a 'vessel of answers' to be tapped into by the interviewer" (p. 369). Here it is assumed that the answers provided by the participant, to the rhetorical questioning imposed by the researcher, simply signifies or designates aspects of the participant's Self. Such a view is indeed contingent upon a naïve view of language. Moreover, the experience of the real person that is ultimately portrayed by adopting such an approach is nothing more than affirmation of the researcher's rhetorical questioning.

Arguably however, at the foundation of micro ethical considerations is what several authors describe in terms of an unbalanced power differential that is inherent within the research interview (Scheurich, 1995; Karnieli-Miller, Strier \& Pessach, 2009; Nunkoosing,

\footnotetext{
${ }^{13}$ Kvale (2007) recalls the work of Charles Perrault (1697/1889) who authored the French version of the children's fable 'Little Red Riding Hood'. Making an analogous link between the qualitative researcher and the 'bad wolf', Kvale (2007) suggests we be aware of the gentle, warm, and caring approaches of interviewers who really wish to invade the private worlds of the interviewees much like the Wolf in the fable. He provides a final caution, that "unfortunately it is the gentle wolves who are the most dangerous of all" (p. 498).
} 
2005; Knapik, 2006, Wengraf, 2001). ${ }^{14}$ This power differential allows the researcher to have what Kvale (2005) describes as "sovereign control" (p. 5). ${ }^{15}$ Here the researcher dictates the avenues opened-up and subsequently pursued during the interview. This power to shape the representations of the respondents produced and ultimately the understanding promulgated of real people and real lives to the wider society, through this control over the interview itself, opens up to challenge the authenticity of the representations produced (Briggs, 2002; Brinkmann \& Kvale, 2005; Gubrium \& Holstein, 2002; Kvale, 2006; Kvale \& Brinkmann, 2009). ${ }^{16}$

So far this discussion has highlighted that the traditional research interview is far less a dialogical encounter than a contrived effort of seeking the respondent's agreement with a series of preordained opinions held by the researcher. The seemingly unavoidable micro ethical issues synonymous with the traditional qualitative research interview lead this thesis to suggest that the representation that is achieved during the interview is not a fair representation of the real lives and real people encountered. Instead, it represents the extent to which the participant is in agreement with the opinions of the researcher.

\section{The Production of Meaning}

It is important to appreciate that any research process subsequent to the interview itself cannot overcome the challenges to representation already alluded to. In fact for this thesis, the necessity to develop meaning after the dialogical encounter itself necessitates the subscription to naïve assumptions about language and meaning and subsequently results in naïve representations of the participants. In line with this, Smythe and Murray (2000) suggest that the key issues concerning the representation of participants "arise during the analysis and interpretation phase of the research, because it is the phase in which the multiplicity of narrative meaning becomes evident” (p. 331). The following passage from Kvale (2006)

\footnotetext{
${ }^{14}$ On the topic of power within the research interview, Wengraf (2001) states, "I wish only to stress that power is a dimension of interview interaction dangerously likely to be overlooked or ignored or denied by the well-intentioned and good-natured interviewer" (p. 45). The ability to unwittingly slip into this power differential is exemplified further by a seam of work that focuses upon the implications of the location of the interview in shaping the power differential within the interview (c.f. Hyden, 1997; Manderson, Bennett \& Andajani-Sutjahjo, 2006; Sin, 2003).

${ }^{15}$ Examining the manipulative nature of the interview, Duncombe and Jessop (2002) suggest that researchers "do rapport by fake friendship" (p. 108), in an effort to get behind, or as Fog (1992) cited in Kvale (2004) states, to "evade ones defences" (p. 156), in the hope of soliciting unguarded information. ${ }^{16}$ Although damning of the state of contemporary approaches to interviewing in contemporary qualitative research, Brinkmann and Kvale (2005) capture the voice of other authors in suggesting that qualitative researchers should seek alternate approaches that make these inherent power relations more transparent. These authors believe an active interviewing approach that is upfront about the role of the participant and does not commodify or instrumentalise the essence of being human may provide an avenue worth pursuing in the serious business of representing the subject matter chosen - the individual person.
} 
highlights the fundamental issue that this thesis has with this phase of the traditional qualitative research process.

In social science research, the interviewer generally upholds a monopoly of interpretation over the interviewee's statements...the research interviewer as the "big interpreter," maintains exclusive privilege to interpret and report what the interviewee really meant and to frame what an interviewee says in his or her own theoretical schemes. (p. 485)

Here then - necessitated by the closing down of the dialogical encounter with the participant - the researcher brings meaning to the words of the participant; meaning that ultimately comes to be the representation of that individual that is presented as understanding of his or her experience. This thesis takes the view that in order to assume the role of imbuing the words of the participant with what he or she 'really meant', the researcher must assume a naïve view of language. A closer examination of thematic approaches for representation within qualitative research further emphasises the centrality of naïve assumptions about language and supports the challenge that qualitative research can no longer claim to legitimately represent the real people and real lives encountered.

Thematic approaches to representation are pervasive within qualitative research. Holloway and Todres (2003) identify "thematizing meaning" (p. 347) as one of the few generic skills across all forms of qualitative analysis. Similarly, Braun and Clarke (2006) also recognise that much of qualitative research analysis is essentially thematic in nature and they go on to suggest that even where a different approach to analysis has been identified - or even where no approach is identified at all - the hallmarks of a thematic approach can be recognised. The path from the research interview to a thematic representation is signposted by a series of procedural steps that are common to qualitative research. A closer examination of each of these steps will highlight the interpretive leaps being made by the researcher at each juncture and emphasise the naïve assumptions about language inherent within the thematic approach that ultimately lead to misrepresentation. The first consideration is the decontextualisation of the interview into a transcript.

\section{From Interview to Transcript}

The process of verbatim transcription is considered to be a benign facsimile of the interview into written form. On the contrary, the process of transcription is a re-production of the interview that is founded upon a failure to recognise the expressive indeterminateness of language. Transcription, in other words, assumes a simple correspondence between the written word and the spoken word, where the written word can represent the sum of the 
observable event itself. Transcription, however, is devoid of the contextually nuanced vibrations of the dialogical encounter itself, and ultimately robs language of its inherently expressive qualities. More specifically, it undermines the participant of the possibility of being represented authentically. ${ }^{17}$ Supporting the view that the transcript is inherently reductionist in nature, Have (1997) emphasised that transcription cannot represent the detail of the dialogical encounter and is instead "always and necessarily selective" (p. 3). Extending this view, Kvale (1996) suggests that in the movement of transforming oral material into written texts, researchers create artificial hybrids impoverished by the lack of contextual information that is only available during the actual dialogical encounter itself. Similarly, Poland (2001) describes the movement into words of the expressively elaborated feelings emotions and values raised during the interview as a process of flattening the richness of the dialogical encounter, a process that does little to further the understanding of the people involved. Picking up on the complex relationship between language and meaning implicit within transcription, Lapadat and Lindsay (1999), Lapadat (2000), Mishler (1990, 1991) and Scheurich $(1995,2003)$ all argue that meaning consists of intractable uncertainties subject to endless re-interpretation, uncertainties that are not overcome simply by the textual accuracy of the transcript. In fact, Downs (2010) holds that, "transcripts are the con artists of the research world" (p. 107), and succinctly captures the view of other scholars in the field who argue that, transcription represents a movement away from the original dialogical encounter with the participant and has implications for the authenticity of any representation (Green, Franquiz \& Dixon, 1997; Lapadat, 2000; Mishler, 1991; Poland, 1995, 2001, 2003; Tilley, 2003a, 2003b).

\section{From Transcript to Thematic Meaning}

In order to highlight the naïve assumptions about language and meaning that are embodied by a thematic approach to understanding, a sketch of what a theme $i s$ and what a theme represents is necessary. ${ }^{18}$ Despite their pervasive use, a clear explication of what a theme is remains elusive. For Strauss and Corbin (1990), themes are those classifications "discovered when concepts are compared one against another and appear to pertain to a similar phenomenon" (p. 61), while for Braun and Clarke (1996) a theme is a consideration of the frequency with which an element occurs within a text or where a pattern is perceived within the data. Frequently occurring elements, that appear to pertain to similar phenomena,

\footnotetext{
${ }^{17}$ It is interesting that Psathas and Anderson (1990), prior to the birth of the iPod generation and the subsequent ease, security and simplicity of digital recording, identified that a transcription is not the data and that the tape recordings of the interviews hold the genuine data and should be listened to over and over again irrespective of the transcription.

${ }^{18}$ For a most insightful look at the history of the 'theme' see: Ryan and Bernard (2003). 'Techniques to identify themes'.
} 
are then grouped together under what Strauss and Corbin (1990) describe as a "higher order, more abstract concept" (p. 61), or a theme. However it is van Manen's (1990) elaboration of the theme that clearly illuminates the seed of a naïve account of language and meaning and underscores the challenge being proposed here regarding the representational legitimacy of qualitative research.

Making something of a text or of a lived experience by interpreting its meaning is more accurately a process of insightful invention, discovery or disclosure - grasping and formulating a thematic understanding is not a rulebound process but a free act of "seeing" meaning. (p. 79)

In each of these definitions the naïve assumption about language and meaning is palpable. To make judgments concerning the extent to which the dialogical elaborations of different individuals appear to be similar implies that the researcher - in order to make such claims is an authoritative interpreter of the meaning that is carried by the words uttered by the participants. Perhaps more blatantly, van Manen's (1990) idea that one is free to see meaning highlights an outright subscription to a naïve view of language and meaning. It is the subscription to such a view that this thesis contends to be problematic. That is, the development of a theme necessitates that the researcher assume that the words of different people carry the same unambiguous meaning. And that the meaning of these words can be freely clustered together in order to develop a thematic representation and that this theme is itself thought to represent an understanding of a person's experience. ${ }^{19}$ Closer examination of the internal processes that inform thematic representation highlight further this naive view of language and meaning. ${ }^{20}$

\section{The Processes of a Free Act of Seeing}

In order to comprehend the widespread implications of the challenge to thematic representation being levelled here, it is important to recall the words of Holloway and Todres (2003) who identify "thematizing meaning” (p. 347) as one of the only generic skills across all forms of qualitative research. Any challenge to thematic representation is indeed a challenge to qualitative research more broadly. The work of Shin, Kim and Chung (2009) identified the most frequently used approach for informing the process of thematic

\footnotetext{
${ }^{19}$ It is these inherent naïve assumptions concerning language and meaning within these qualitative approaches that encourages this thesis to reach toward the further promotion of the dialogical opportunity within qualitative research.

${ }^{20}$ A more specific exploration of the deeper elements of Colaizzi's (1978) approach to data analysis will be undertaken in Chapter Seven. The landmarks of Colaizzi's (1978) approach, which are common within contemporary qualitative research will provide a framework for considering the advances made by the new theoretical approach being developed here.
} 
representation that researchers using a phenomenological research approach adopt. These authors found that the work of Colaizzi (1978), van Manen (1990), Giorgi (1985) and Smith (1996a) represent the most frequently adopted theoretical positions in the development of a theme. Thus, in order to facilitate a consideration of the - surprisingly consistent - internal processes that inform thematic analysis, a consideration of these processes, as proposed by each of these scholars, will be used as a vehicle for drawing similarities between their respective approaches. In addition, these similarities highlight the pervasive nature of the naïve assumption about language and meaning that can be seen within thematic analysis and underscore the questions being levelled by this thesis concerning the authentic representation of real people and real lives in qualitative research.

As a point of significant similarity each of these approaches suggest that the researcher make determinations of the meanings embedded within aspects of the interview material that he or she deems to be significant. In achieving this, Colaizzi (1978) suggests that the researcher extract 'significant statements' from the transcript, while Smith (1996a) uses the word 'themes' to identify the process of locating significant material for analysis. Giorgi (1985) proposes that the researcher 'create units of meaning by intuitively making sense of the data' and Van Manen (1990) on the other hand requires that the researcher 'compose linguistic transformations' and thereby transform the words of the participants into more meaningful or sensitive paragraphs. Giorgi (1985) also suggests that the researcher transform the meaning units into meaning statements, and Smith (1996a) and Colaizzi (1978) ask that the researcher identify those aspects of the previously identified significant statements that are of most pertinence and to subsequently draw meaning from them.

Despite the subtle variation, the adherence to a naïve view of language and the subsequent idea that meaning is somehow transparent is ever present. Firstly, suggesting that the researcher is able to identify those aspects of other people's dialogue that are meaningful, raises the question 'meaningful for whom'? In addition, suggesting that the researcher uncover or intuit the meanings that are assumed to be embedded within the words of a participant - after the dialogical encounter itself - is a further and clear subscription to a naïve assumption concerning language and meaning. Once the meaning of the participants' statements are made, each of these authors suggest that the meaning units be clustered (Colaizzi, 1978), connected or composed (Smith, 1996a; Smith, Flowers \& Larkin, 2009), or amalgamated together in such a way so as to reveal a 'structure' (Giorgi, 1985). For each of these scholars, these structures of clustered meaning culminate in what is considered to be as complete a representation as possible of the experiences of the participants involved. Thus, it is clear here that each movement of understanding within a thematic approach is a movement 
- directed exclusively by the researcher - progressively further and further away from the account originally provided in the dialogic encounter with the participant him or her self.

This consideration of the thematic approach emphasises that the processes that are common to thematic representations - the same processes that permeate qualitative research are in fact informed by a naïve assumption about language and meaning and have widespread implications for representation within qualitative research. That is, in viewing these internal processes of thematic analysis and the subsequent representation of the participants, the monopoly of the researcher over the representation that is produced is self-evident. This dominance of the researcher challenges the claim that qualitative research can legitimately make to the representation of the real lives and real people encountered. Although the following passage from Scheurich (1995) is predominately based in metaphor, he is able to capture the interpretive leaps that are here being alluded to within the movement of meaning making by the researcher.

The decontextualized interview text which is transformed through the coding process becomes that from which the conventional researcher constructs his or her story. The bricks of the construction are the reductive nomads of meaning, coded in categories in the transcripts. These bricks are formed, however, from a mold that is then shaped from the researcher's conscious and unconscious assumptions and orientations... all of the juice of the lived experience has been squeezed out...while these generalizations are said to represent reality, in my view they mostly represent the mind-set of the researcher. (p. 241)

Given then that Holloway and Todres (2003) have highlighted thematic analysis to be the mainstay of developing understanding within qualitative research - a series of processes that are founded upon a naïve view of language and meaning - then the extent to which any understanding achieved is indeed an understanding of the participant(s) involved is open to question. The naïve interpretive leaps that are necessitated by the adoption of a thematic approach operate to ultimately undermine what comes to be understood of the subject matter of focus.

Nonetheless, in challenging this process of interpretation, this thesis is not suggesting that interpretive-free understanding is a human possibility. ${ }^{21}$ Rather it proposes that the

\footnotetext{
${ }^{21}$ It must be noted that interpretation is for qualitative research perhaps the greatest testament and yet its greatest challenge. Vehemently opposed by the positivist paradigm, interpretation is through the lens of such a position considered relativist and inherently unscientific. However, Gadamer
} 
discipline of qualitative research needs to critically examine what it is that is actually being interpreted. This exploration of the internal processes of thematic analysis has shown that what is actually being interpreted is firstly, those aspects of the participants dialogue that the researcher considers to be most significant, and secondly, what it is that the researcher believes that the participant really meant by those statements. Significantly the understanding carried forward in the research literature is therefore open to the challenge of only poorly representing the experiences of real people and the real lives encountered. On this basis, qualitative research should consider the way in which the meaning of the phenomenally experienced person is carried forward and promulgated. A closer look at the way the meanings developed by the researcher are carried forward is useful.

\section{The Carriage of Meaning}

In the same way that Copernicus placed the sun rather than the earth at the centre of the universe, modern qualitative research has placed the 'researcher's interpretation' instead of the 'subject matter of focus' at the centre of the endeavour of understanding. ${ }^{22}$ The discussion thus far has highlighted that placing the researcher at the centre of the process of understanding the real lives of other people necessitates a subscription to a series of assumptions concerning language and meaning that ultimately have implications for the understanding that is carried forward.

Although qualitative researchers seek individuals who are sufficiently experienced with the phenomena of interest to be involved in their studies, the movement of understanding that transpires after the dialogical encounter makes us wonder about the degree of value that is actually placed upon the participant's experiences. For example Charmaz (2004), who can be seen as clearly subscribing to the naïve approach being challenged here, suggests that in thematic analysis a researcher should be "excavating the implicit meanings in our participants' statements and actions” (p. 986). However, this movement of excavation represents merely a series of 'guesses' as to the meaning of the participant's words and highlights the limited value that is actually placed upon the dialogue between researcher and

(1960/2003) in his work Truth and Method challenged the notion that understanding could ever be free from interpretation. Here he provided one argument - amongst others - that 'scientific truths' are but interpretations. At some point a 'scientist' must make an interpretation about what it is that a thing is showing to be the case. Although not fully fleshed out here, Gadamer was able to highlight that understanding is indeed interpretation. Gadamer (1960/2003) also established by way of his broader Hermeneutic Phenomenology that "interpretation is not an occasional post facto supplement to understanding; rather, understanding is always interpretation, and hence interpretation is the explicit form of understanding” (p. 307). Significant recourse will be made Gadamer's (1960/2003) work Truth and Method in Chapter Five.

${ }^{22}$ Regarding the links here with Copernicus's ideas and a consideration of the centering of the human subject initiated by Kant and arguably perfected by Hegel refer: Redding (1996). Hegel's Hermeneutics. 
the participant. Instead, the 'guesses' generated of one participant's experience are amalgamated with the 'guesses' developed of another participant's experience - participants who are necessarily perceived to be homogenous. And in aggregate these guesses form a theme that culminates in what comes to be the totality of the understanding of the individual participants involved.

Although the use of the term 'guesses' may appear to be an offhand critique, the term 'intuiting', a notion that commonly informs the process of thematic analysis is less than complementary when elaborated. Intuition "is the apparent ability to bring to or acquire knowledge without inference to or the use of reason" (Oxford English Dictionary, 2000). To 'intuit' something is to make meaning or bring to understanding a thing by habit. ${ }^{23}$ Therefore, the movement of intuiting represents an effort to make meaning of the experiences of another person by appealing to a gut feeling, devoid of reason, and in total isolation from any ongoing dialogue with that specific person. ${ }^{24}$ The open acknowledgement of subscribing to the process of intuiting by researchers is an effort to be seen to be doing what is right or what is common sense in making a decision that leads to action. This thesis does not take issue with intuiting such things as how switch on a new oven or making a decision to turn left or right, or even taking a child to the physician believing he or she is ill. However, in the movement of qualitative research we are not dealing with acts but with experiences, emotions, thoughts, feelings, and the qualities of the innermost experiences or qualia of other people. Each of these individual people have idiosyncratic psychological processes and therefore unique lived experiences. It is therefore argued that these qualia cannot be known or represented in a meaningful way by appealing to that which one 'guesses' to be 'the right thing to do'.

As well as highlighting the pervasive subscription to a naïve view of language and meaning within qualitative research, the adherence to a 'common sense' or 'intuitive' approach to representation perpetuates the representation of the glaringly obvious. Underpinning this view is a belief that thematic representations are only capable of operating at a superficial level of data abstraction. That is, in order to conjure themes that are considered to represent those involved in a study, one must take a broad perspective. Although at this broad level of consideration participants may be able to identify similarities

\footnotetext{
${ }^{23}$ Although it is not a position subscribed to by this thesis Rosemarie Anderson developed a transpersonal research method titled 'Intuitive Inquiry' which invites intuition into the research practice of 'Embodied Writing'. For a more thorough review see: Anderson (1998) 'Intuitive inquiry: A transpersonal approach'. In Braud and Anderson. Transpersonal research methods for the social sciences: Honoring human experience.

${ }^{24}$ Contrary to this view Janesick (2001) advocates the use of intuitive and creative practices in an effort to "shift the conversation about qualitative research methodology and design from the 'McDonaldization' or 'Disneyfication' of method and design to an understanding of the intuitive and the creative" (p. 532).
} 
between their own experiences and some aspects of the thematic representations developed, at this level of abstraction these representations cannot be considered to be a complete or even a faithful representation of each individual person encountered.

In order to elaborate this view, similarities between a thematic representation and the operation of the website Google Earth can be made. Containing archival satellite imagery of the world with amazingly clear angle reduction or zooming properties, the website enables the user to select a geographical focus for closer examination. Here if you wished to focus upon your own house you may be able to recognise clearly your garage, the children's toys in the back garden, the sandpit or even the clothesline. The detail at this level is quite remarkable and the unique elements that make your house your own are easily noticed. If you decided to view the wider municipality or township you would zoom-out from your yard to the broader town. At this level you can see perhaps a large expanse of water, a noticeably main road, or an industrial or retail area in such a configuration as to be indicative of your hometown. However, at this level your own home may appear as merely a tin roof, one amongst many other tin roofs and generally unrecognisable from the others. Here you can see that 'yes' you do have a lot in common with the rest of your town in that you too have a tin roof the same colour as the majority of others and that you have a very similar yardage as your fellow community members. But at this level the idiosyncratic aspects of your own yard are indiscernible, lost to the broader picture of the masses or the generality of your township by the zooming-out from the detail that is possible.

Within this analogy is a consideration of the way in which we can legitimately be considered to authentically represent the individual(s) involved in qualitative research, when the primary focus of concern is at the level of the wider more superficial level of generality. This position closely echoes that of Seale, Gobo, Gubrium and Silverman (2004) who suggest that qualitative researchers often produce "low quality research and research results that are quite stereotypical and close to common sense" (p. 2). It is clear that the zooming-out from your unique backyard - analogous to your inherently unique experiences - to the more 'general' aspects of a shared society carries with it a loss of attention to detail, a loss of idiosyncrasy and a change in the depth of focus. Herein lies the core challenge against contemporary qualitative approaches. In the search for 'generality' as a theme, among or across a group of people, all of whom are considered to be homogenous, the discipline of qualitative research has sacrificed a tremendous depth of understanding of the real people and real lives encountered. Instead, those within the domain of qualitative research have perpetuated a number of assumptions that operate to challenge the ability of contemporary 
qualitative research practice to represent that which is to be represented - the individual human being.

\section{The Research Presentation}

The final familiar signpost of the qualitative research process is the presentation of the representations developed by the researcher. Here the implications of an assumed naïve transparency of language and meaning are writ large. For several authors the monopoly that the researcher has over the process of meaning making and ultimately the account of the individual that is presented, opens qualitative research to the challenge of presenting little more than sheer relativism (Kvale, 1996, 2006; Kvale \& Brinkmann, 2009; Richardson, 1994, 2000; Rosaldo, 1993; Smith \& Sparkes, 2008, 2009; Sparkes, 1995; Tierney, 1993). It has been established that the thematic representations developed by the researcher - borne out of a necessity for adhering to naïve assumptions about language and meaning - form the account that is ultimately presented. Upon this basis, the claim to relativism made by these scholars sits comfortably with the argument levelled by this thesis.

Within the presentation of qualitative research, the naïve assumption about language and meaning is most often played out through the presentation of frequent and lengthy - and therefore supposedly accurate - quotations from participants in an effort to represent a connection with reality (Lee, 2001; Johnson, 1997). According to van Maanen (2011) qualitative researchers work meticulously to be perceived as being "fully able to whistle native tunes" (p. 64). Despite this, he goes on to suggest that the use of these lengthy, grammatically incorrect and at times incoherent quotations from participants' interviews as evidence for this competence ultimately represents a corruption of representation. ${ }^{25}$

Suggesting that the use of these quotations is staged, van Maanen (2011) argues that they are surreptitiously edited in such a way as to represent and emphasise the researcher's perspective, all the while using what appears to be the views of the participants. While the voices of participants are here present within these seemingly 'realist tales', they are none the less designed to serve the needs of an absent and disembodied author (Sparkes, 1995). ${ }^{26}$

\footnotetext{
${ }^{25}$ According to Brinkmann and Kvale (2005) "Ever since David Hume's observation in the early eighteenth century, that one cannot logically infer evaluative judgements from descriptions, there has been a sharp separation of fact and value in Western thought" (p. 158). This separation could be argued as being the vehicle for a penchant within qualitative research for establishing a sense of legitimacy. So much so, that any consideration of the notion of representation within qualitative research must at some level seek to tie-down the notion of 'a real'.

${ }^{26}$ Although this thesis is concerned with the pervasive subscription to naïve assumptions about language and meaning that is necessitated by the closing down of the dialogic opportunity with the participant, there is also a body of work concerned with the way in which the use of literary strategies perpetuate the researcher's representational monopoly. Sparkes (1995) suggests that "scientific tales
} 
Thus, regardless of attempts to tuck the researcher within the folds of the research by the use of literary devices, it is merely an illusion. Sparkes (1995) captures the ever-present determinacy of the researcher in the following passage:

It's the author whose guiding hand selects the quotations and shapes the story presented...my disappearance needs to be seen as a textual strategy, a conscious decision to focus attention upon the subject's words with a view to drawing the reader into the storyline... and evoking a response. (p. 170)

With the power to write people into and out of the final product, qualitative researchers are clearly implicated in the construction of the final text and have an ethical obligation to represent the real people and real lives encountered as genuinely as possible, and to produce faithful representations that exceed any requirement to be simply a 'good read'. ${ }^{27}$ The written product can no longer be seen as an innocent or neutral element in the movement of understanding. Rather, it is the platform through which naïve assumptions about language and meaning and the subsequent implications of these assumptions for the representation of the experiences and lives of real people are promulgated to the wider community. In fact for this thesis, the implications of these naïve and pervasive assumptions, cast a specter of doubt

constitute very powerful and persuasive fictions in western cultures" (p. 165), such that their pervasive use is an unsurprising strategy within the domain of a qualitative research that continues to seek scientific legitimacy. Despite the suggestion made by Smith and Sparkes (2009), that the centrality of the researcher in qualitative research represents a moral issue for the discipline of qualitative research, this thesis argues that qualitative researchers regularly engage in what Strathern (1990) first alluded to as Persuasive Fiction. Here a researcher uses literary strategies to draw the reader into the story being told, and persuade them of its representative capacity. Smythe and Murray (2000) draw attention toward what they term 'Narrative Ownership' and the capacity that the researcher has to determine the presentation that is ultimately presented. These authors go on to suggest that the greatest risk to participants involved in qualitative research is the risk of having his or her story reinterpreted and filtered often through the "lenses of social-scientific categories" (p. 321). Perhaps the most significant implication of these naïve relativistic representations is what Smythe and Murray (2005) describe as a concern "that the researcher's account fails to jibe with participants' views of themselves; it fails to capture their sense of their own individuality and uniqueness" (p. 184). Sparkes (1995) has also argued that the ways in which the researcher chooses to write have profound implications for that which is represented by the research, including such elements as the way in which a person's views are given priority or weight, and ultimately the way in which that person's reality is presented. Undeniably therefore, the researcher is afforded the power of providing what Apter (1996) has termed the "expert narrative" (p. 42), which for several authors opens up the possibility of negative reactions in participants who believe that they have been poorly represented (Bakan, 1996; Bar-On, 1996; Smythe \& Murray, 2003). The words of Chase (1996) most completely capture these claims: "If a participant expects that the researcher will capture fully who she is, then it must be disconcerting to have her story analyzed for the social processes it reveals rather than preserved in its uniqueness" (p. 50).

${ }^{27}$ Smythe and Murray (2000) make some insightful critiques of conventional approaches to qualitative research with a particular focus upon the ethical issues inherent within qualitative studies. These authors problematise a series of often neglected issues such as: consent (what is actually being consented to in qualitative research), deception (the guiding of a narrative interaction toward a predetermined point), data source models (treating participants as sources of data that are merely lifted from them), as well as privacy and anonymity (problematic when producing texts often richly filled with unavoidable identifying markers). 
over the representations of experience already portrayed through previous qualitative research. $^{28}$

In seeking to draw a conclusion to this initial discussion, concerning the way in which the pervasive adherence to a naïve assumption about language and meaning, and the profound implications of the claim to the authentic representation of the experiences of real people that can be made by qualitative research, it is useful to reiterate the question from which this discussion in part is a response.

Is it possible that qualitative research has contributed less than would seem attainable in the study of individuals in light of the fact that qualitative researchers have been working on the basis of a number of assumptions about people, which have not been examined very carefully, and have been adopting a number of assumptions about the nature of science which are less than adequate for the subject matter chosen as the focus of investigation?

The assumption that words carry with them unambiguous meaning that is directly accessible to the researcher challenges the capacities of qualitative research to genuinely represent the real people and real lives encountered. ${ }^{29}$ This thesis suggests however that the perpetuation and pervasive acceptance of these assumptions about language characterises an effort by qualitative researchers to 'wish' the 'representational gap away'. ${ }^{30}$ In other words, by

\footnotetext{
${ }^{28}$ Although the primary focus here is the 'representational capacity' of qualitative research itself, it provides a suitable juncture to signpost the unethical nature of the misprepresentation that is argued as being inherent in qualitative work. According to Brinkmann and Kvale (2005), qualitative research can be considered "saturated with ethical and moral issues" (p. 157), issues that for Mauthner, Birch, Jessop and Miller (2002) originate by way of researching and making public otherwise private lives of individuals. The ethical dilemmas that are inherent in qualitative research (Fuentes, 2004; Hadjistavropoulos \& Smythe, 2001; Ponterotto, 2005) lead Guillemin and Gillam (2004) to encourage the qualitative researcher to be attuned to the "ethically important moments" (p. 262) of the endeavour. Despite these arguments, several 'key' authors within the qualitative field believe qualitative research approaches are inherently democratic and ethical in nature (Denzin \& Lincoln, 2005; Hammersley, 1999). Several authors argue that the reciprocity that is inherent within the research interview leads to qualitative research being considered as ethically sound (Brabeck, 2000; Christians, 2000; Mauthner, Birch, Jessop \& Miller, 2002). Despite what may at first appear as an 'egalitarian turn', the theoretical assumptions embodied by what Smythe and Murray's (2000) describe as 'Narrative Ownership' give rise to ongoing challenges concerning the representational nature of qualitative research and the subsequent ethics of misrepresentation.

${ }^{29}$ Scholars of the qualitative research arena have conducted limited research with a focus upon the 'novel' meaning making aspects of representation. Despite this, a growing scepticism concerning representation can be seen within the literature on psychotherapeutic intervention, perhaps most notably within the literature concerning family therapy. For an overview of these considerations see: Hare-Mustin (1994) 'Discourses in the mirrored room: a postmodern analysis of therapy', or Kogan (1998) 'The politics of making meaning: Discourse analysis of a 'postmodern' interview'.

${ }^{30}$ In line with the issue of 'wishing the gap' away, Mantzoukas (2004), suggests that - despite the centrality of the concept of representation within research - qualitative researchers offer very little if
} 
subscribing to a designative account of language the inherent indeterminateness of language is overcome and the researcher can surreptitiously paper over the gap between who should be represented and who indeed is represented in the final product.

In fact this thesis suggests that the representational legitimacy of a piece of research is representative of the degree that this representational gap is overcome. That is, the way in which the experiences of real people are presented and not simply those of the researcher. Here legitimacy is a consideration of the extent that the naïve assumptions about language and meaning that are necessitated by contemporary approaches to qualitative research, would be overcome through reinstating the centrality of dialogue in all understanding. However, within contemporary approaches to qualitative research considerations of legitimacy are embodied by what is commonly referred to as the 'Criteria Debate'. A sketch of this will provide further evidence for the often unchallenged acceptance of these inherent and naïve assumptions about language that call into question the claim by qualitative research to authentic representation. It will also provide an overview of the milieu within which the theoretical position that this thesis ultimately develops will have to contend with.

\section{The Criteria Debate}

Producing justifiably robust representations of the lives and experiences of individuals is further complicated by a lack of - or even perceived need for - consistent judgment criteria. In other words, the notion of representation within qualitative research is plagued by the lack of a consistent touchstone to make determinations of the value of research outcomes. Ultimately this value is a consideration of the degree of representational legitimacy that a piece of qualitative research is able to lay claim to. It has been suggested within this thesis that the representation of the qualia of an individual person should be the most fundamental focus of consideration for qualitative research. The previous discussion however, highlights a lack of consideration by researchers for the implications of naïve assumptions about language and meaning. The pervasive nature of these practices, as underscored by a consideration of thematic representation, challenges the claim to representational legitimacy that qualitative research can make. At the heart of this challenge to legitimacy, are ultimately considerations of the notion of validity, ${ }^{31}$ or the extent to which a researcher can claim to have represented 'a real'.

any analytical consideration on representational issues in their end texts at all. Questions such as whom or what is being represented by an investigation are rarely addressed.

${ }^{31}$ According to Kvale (1996), validity is simply one element of a wider "Scientific Holy Trinity" (p. 229) within the scientific community. The elements of this trinity include validity, reliability and 
Validity usually refers to the truth and the correctness of a statement (Kvale, 1996; Kvale \& Brinkmann, 2009). For Silverman (2000), the notion of validity as truth is fundamental to the practice of qualitative research given that "an account is valid or true if it represents accurately those features of phenomena that it is intended to describe, explain or theorise" (p. 175). In another way, validity is therefore the determinant by which a researcher can be seen to accurately represent those features of phenomena that the original study intended to. Silverman (2000) goes on to argue that without an appeal to a notion of validity, qualitative research descends into a slanging match where the only winner is the one "who shouts the loudest" (p. 175). Thus, these ongoing quandaries ultimately concern the way that researchers situate their research with regard to a real. Embodied by what has come to be known as the 'Criteria Debate' is the conjecture that surrounds an appeal to or rejection of the realism that is implied by notions of validity.

In an article titled 'Myth \#94: Qualitative health researchers will agree about validity', Sparkes (2001) offers a considered exploration of the broader theoretical complexity that surrounds the notion of validity within qualitative research. Here Sparkes (2001) usefully reins in this complexity by identifying three distinct, factionist threads within the literature The Replication Perspective, The Parallel Perspective and The Letting-go Perspective. ${ }^{32}$ An examination of this debate, highlights what Silverman (2000) describes as the "methodological anarchy" (p. 290) present within the discipline, and further problematises the claim to representational legitimacy that can be made by qualitative research. Each of these threads identified by Sparkes (2001) will be used as a signpost for a closer consideration of the Criteria Debate itself.

\section{Replication Perspective}

Adopting a realist position, the replicative perspective takes the view that it is a real world that our representations are of and that it is possible for these representations to be presented in a valid way. Authors within the replicative perspective believe it is possible to develop criteria for making determinations about the validity of these representations. These criteria would be used to make determinations of the degree to which the understanding

\footnotetext{
generalisability. Reliability is concerned with the consistency of the research findings, or the extent to which the researcher can be guaranteed to achieve the same results if the study was conducted again. Generalisation is a consideration of the extent to which the findings from a study can be considered commensurate with other subjects and situations. Although each of these 'canons' will be the focus of a later discussion, suffice to say here the latter two criterion are routinely overlooked by the broader Criteria Debate in favour of an almost exclusive consideration upon validity.

${ }^{32}$ Sparkes (2001) refers to four distinctions within the criteria. That is, as well as the Replication, the Parallel, and the Letting-go perspective, he also describes what he terms the Diversification perspective. The central features of this later perspective have been amalgamated within the discussion concerning the Parallel perspective in the discussion provided here.
} 
produced is representative of the subject matter of focus. It would be unfair to anticipate that these replicative adherents could engage in the same mathematical manoeuvres employed by their quantitative counterparts, whereby the degree of validity according to which we can have confidence in the results can be established. Despite this, the replicative perspective is founded upon a subscription to the same core principles embedded within these traditional notions of validity. Thus, replicative thinkers suggest that the same criteria for making determinations of a study's value or validity should be replicated across all forms of research (Barker, 2003; Dixon-Woods, Bonas, Booth, Jones, Miller, Sutton, Shaw, Smith \& Young, 2006; Dixon-Woods, Shaw, Agarwal \& Smith, 2004; Silverman, 2009; Teddlie \& Tashakkori, 2003).

Concerned with a widespread shift away from the notions of validity by the discipline of qualitative research, Morse (1999a) wrote:

To state that reliability and validity are not pertinent to qualitative inquiry places qualitative research in the realm of being not reliable and not valid. Science is concerned with rigor, and by definition, good rigorous research must be reliable and valid. If qualitative research is unreliable and invalid, then it must not be a science. If it is not a science, then why...should it be taken seriously? (p. 717)

Echoing these sentiments, Clavarino, Najman and Silverman (1995) invite qualitative researchers to confront the challenge that qualitative research is merely exploratory, relativistic and a journalistic 'soft-science' filled with personal bias. In contrast to this view, these authors suggest that scholars should consider approaches to qualitative data analysis that are no less concerned with reliability and validity than their quantitative counterparts. In fact, they go on to suggest that claims against the tenets of the replicative perspective are replete with little more than "emotive rhetoric" (p. 224), that operates to destabilise the discipline. These authors argue that subscribing to notions of reliability and validity will ultimately move the discipline of qualitative research forward. Thus, in order to avert a slippage into the 'anything goes' anarchism alluded to by Silverman (2009), Clavarino, Najman and Silverman (1995) argue that qualitative research must locate itself within the domain of realism. The following passage is exemplary of this view.

In this sense, we accept the "postpositivist" position that scientific criteria should be fitted to a "naturalistic" research context. This means that we desire to retain generalizable findings and scientific credibility while 
rejecting humanistic appeals to "authenticity" and the siren calls of relativism (p. 225).

These authors go on to suggest that as well as the possibility of overcoming the paradigm war - evident here within the broader criteria debate - agreement about notions of validity would help to strengthen the scholarship within the discipline of qualitative research further.

Despite the productive contribution of valid representations to the scholarship of qualitative research, the replication perspective is not immune to critique. At the centre of this critique is an argument that the replication perspective is beholden to an untenable assumption concerning the existence of a single reality (Smith \& Hodkinson, 2009; Sparkes \& Smith, 2009). The following passage from Smith and Hodkinson (2009) encompasses the thrust of the argument against replicative approaches.

There is no way to 'get at' that reality as it really is. And, if one cannot capture that social reality as it really is, then that reality cannot be called upon to do the adjudicate-the-different-claims-to-knowledge work asked of it. That is the whole problematic posed by the idea that no matter how hard we try, we cannot achieve theory-free observations or knowledge. (p. 34)

It is clear that for these authors, notions of validity only become a possibility if a single fixed reality can be shown to exist. This fixed reality therefore provides the backdrop against which judgments of right or wrong can be made. ${ }^{33}$ However, this argument assumes that validity can logically be represented in terms of an off/on modulation. In other words, something is either valid or it is not. However, such a conceptualisation does not embody the notion of validity as a means of judging the degree to which a person's reality - 'a real' - is represented. Instead, it is concerned only with the presentation of a fixed reality - or 'the real'. To clarify this position the following exemplar is helpful.

The conduct of research concerning the experience of children who have nightmares may well yield a discussion concerning a fear of monsters, particularly the ones with the big blue spots. In single fixed reality, monsters do not exist. However, a representation of these monsters as having 'big blue spots' is nonetheless valid in that it represents the actuality of the subject matter - the child's experience of his or her nightmare. By subscribing to a notion of validity where the only things that can be judged as correct are those things that can be

\footnotetext{
${ }^{33}$ On this issue Sparkes and Smith (2009) contend that attempting to establish this position "attempts to square a philosophical circle" (p. 493).
} 
tied-down within the world - a naïve realism - is to deny the qualitative research paradigm the possibility of contributing legitimately to an understanding of being human. ${ }^{34}$

\section{Parallel Perspective}

The underlying premise of the parallel perspective is that qualitative and quantitative research represents alternative paradigms. On this basis, each paradigm requires a different series of specific judgement criteria. Thinkers within this perspective challenge the philosophical confluence of replicative approaches with qualitative research. Instead, they argue in favour of the parallel perspective given its acceptance of the existence of multiple realities (Angen, 2000; Denzin \& Lincoln, 1994; Ellis, 1999; Lincoln \& Guba, 1985; Pyett, 2003). Yardley (2000) effectively captures the assumptions that underpin the parallel perspective in the following passage.

The unwillingness of qualitative researchers to converge on a unitary set of methods, assumptions and objectives can lead to confusion and scepticism about the validity of their work. But a pluralist ethos is central to the nonrealist philosophical traditions underpinning most qualitative research...But if this is the case, there can be no fixed criteria for establishing truth and knowledge, since to limit the criteria for truth would mean restricting the possibilities for knowledge, and would also privilege the perspective of the cultural group whose criteria for truth was deemed 'correct'. (p. 217)

Although Yardley (2000) acknowledges that a lack of well-defined and agreed upon criteria for making judgments about the quality of qualitative research makes the discipline vulnerable to a number of challenges, ${ }^{35}$ she suggests "most qualitative researchers reject the idea that there ever could or should be a universal code of practice" (p. 217). Thus, in response to a widespread suspicion of approaches that adopt positivist standards for making determinations of the value of qualitative research, ${ }^{36}$ several authors have sought to develop

\footnotetext{
${ }^{34}$ The discussion concerning naïve realist conceptions of validity will be taken-up again in Chapter Eight and will form the foundation for a consideration of validity from the vantage point of the theoretical approach established through this thesis.

${ }^{35}$ Perhaps the most significant challenge identified by Yardley (2000) is the risk that qualitative research becomes fragmented into those approaches that are able to address the traditional criteria and those that cannot. Despite the representational benefits that would result from an adherence to these traditional criteria, Yardley (2000) is concerned that such an approach might inhibit the "more radical methodologies" (p. 218) from engaging with the discipline.

${ }^{36}$ Examples of work that supports a shift to the Parallel perspective citing incommensurate theoretical differences between qualitative and quantitative positions can be seen in Angen (2000); Denzin \& Lincoln (1994); Ellis (1999); and Pyett, (2003).
} 
flexible criteria. These criteria are supposedly more consistent with the interpretive presuppositions of qualitative research (Yanow \& Schwartz-Shea, 2006).

Arguably responsible for the inception of the parallel perspective into the domain of qualitative research, is the work of Miles and Huberman $(1984,1994)^{37}$ and Lincoln and Guba (1985). Following the work of Lincoln and Guba (1985), ${ }^{38}$ as outlined through their text Naturalistic Inquiry, we find a critique of the infusion of positivism into the qualitative (naturalistic) domain. In response to this critique, Lincoln and Guba (1985) propose a series of essential propositions or "axioms of the naturalistic research paradigm" (p. 36). Of the fourteen axioms elaborated, the one that is most pertinent to this discussion is titled 'Special Criteria for Trustworthiness'. The following passage encompasses clearly this axiom.

$\mathrm{N}$ [the naturalist] is likely to find the conventional trustworthiness criteria (internal and external validity, reliability, and objectivity) inconsistent with the axioms and procedures of naturalistic inquiry. Hence he or she is likely to define new (but analogous) criteria and devise operational procedures for applying them...the case will later be made that there exist substitute criteria called credibility, transferability, dependability, and confirmability together with corresponding empirical procedures that adequately (if not absolutely) affirm the trustworthiness of naturalistic approaches. (pp. 42-43, emphasis added)

The criteria of credibility, transferability, dependability and confirmability, which are considered to be determinant of a study's trustworthiness, have gained widespread appeal within the qualitative discipline. However, it is the use of the word substitute that highlights most clearly the contradiction inherent within this approach. A brief sketch of the parallels between Lincoln Guba's criteria and the traditional canons of validity is elaborative of this argument. $^{39}$

\footnotetext{
${ }^{37}$ Miles and Huberman did not provide a fully-fledged discussion of their criteria for qualitative research until the second edition (published 1994). However, their earlier work (published 1984) accepts the critique by positivism of the unreliable and biased state of qualitative analysis. These authors propose a redress of this critique by following a series of techniques. However, these techniques are the same processes that inform thematic representation and are therefore open to challenge as outlined in this chapter.

${ }^{38}$ Lincoln and Guba's (1985) work has been followed here as an exemplar. Miles and Huberman (1994) also use the principles of these authors' work as a foundation for their own work. So much so that Miles and Huberman (1994) cite Lincoln and Guba's (1985) evaluative criteria: Credibility, Transferability, Dependability and Confirmability and later identify their evaluative criteria for qualitative research as: Authenticity, Fittingness, Auditability, Confirmability and Action.

${ }^{39}$ Several other authors provide considered critique of the criteria with which Lincoln and Guba (1985) suggest that Trustworthiness can be established. Elaborated as "the most crucial technique for
} 
Lincoln and Guba (1985) go to great lengths to make evident the correlations that exist between internal validity and credibility, external validity and transferability, between reliability and dependability and objectivity and confirmability. ${ }^{40}$ Here the influence of the supposedly inconsistent - positivist criteria for validity, upon the notion of trustworthiness is palpable. In fact, merely substituting one set of terminology for another is simply a surreptitious subscription to validity in another guise. ${ }^{41}$ As captured by the words of Sparkes and Smith (2009), Lincoln and Guba (1985) "on the one hand paid lip service to ontological relativism but on the other they espoused epistemological foundationalism in the form of procedures or method to sort out trustworthy interpretations of reality" (p. 493). Thus, this new - or rather analogous - series of criteria becomes the backdrop against which determinations of what matters in qualitative research are based. In fact these analogous criteria represent a clear contradiction to the fundamental principles that inform the parallel perspective itself.

More recently, Hammersley (2009a) ${ }^{42}$ proposed another series of supposedly alternate criteria for making determinations of the value of research. He described 'plausibility' as "the extent to which a new knowledge claim follows on from, or at least is not incompatible with, what is currently taken to be sound knowledge" (p. 16). He went on to describe 'credibility' as "whether or not the evidence is open to reasonable doubt because of the way it is produced - whether error seems likely to have been involved" (p. 16). ${ }^{43}$ In his

establishing credibility" (p. 14), the 'Member Check' or the returning of the interview transcript to the participant for review has come under a sustained critique. Several authors (cf. Bloor 1983, 1997; Gallagher, 1995, 2001; Horsburgh, 2003; and Silverman, 1993, 2000, 2009) suggest that the essential logic of qualitative research is to capture the responses of participants that are never immaculately produced and are but one representation amongst many in one moment in time.

${ }^{40} \mathrm{On}$ the clear synergies between traditional conceptions of traditional validity and those of Lincoln and Guba (1985), Manning (1997) wrote, "trustworthiness was conceived as a parallel to the empiricist concepts of internal and external validity, reliability and objectivity" (p. 95).

${ }^{41}$ It must be noted that Lincoln and Guba (1989) reflected upon their work and responded to criticism by recognising the foundationalist notions embedded within their approach. They were concerned that their work had been considered in terms of being a method over and above an avenue for ensuring a participant was faithfully represented. In their more recent work Guba and Lincoln (2005) demonstrate their shift away from the previous foundationalism within their work by an appeal to two criteria they term 'ontological' and 'educative' authenticity. Although the elaboration of these positions is considered beyond the scope of the aims established for this section, it is enough to suggest that these authors have shifted their own thinking away from their earlier (1985) work. According to Sparkes and Smith (2009), this raises several questions concerning the non-reflective adherence to their earlier position and criteria by many researchers conducting qualitative research and more significantly who judge the value of qualitative research more broadly.

${ }_{42}$ It is worth noting here that in earlier publications, Hammersley $(1990,1991,1992)$ identified 'Relevance' as a fundamental criterion for determining the value of qualitative research. Relevance was accepted in terms of the importance of the research topic and the contribution to knowledge of the findings generated.

${ }^{43}$ It is important to make clear that Hammersley's (2009a) notion of 'plausibility' is not simply an acceptance of those knowledge claims that match a researcher's own existing knowledge. A view such 
effort to stave off relativism, Hammersley (2009a) adheres strongly to the view that there are indeed real phenomena independent of the researchers' accounts of them and that by adhering to explicit procedures we can produce knowledge. ${ }^{44}$ Once again however, the parallels between these so-called alternate criteria and those of the replicative perspective are evident. Indeed, Hammersley's (2009a) position is open to the same critique as that levelled at the work of Lincoln and Guba (1985).

In what is best described as a slippage into a tautological position, Scheurich (1996) highlights the way in which the replicative and parallel perspectives are decidedly similar. At the centre of notions of validity are discriminations between research that is good and research that is not good. Although scholars within the parallel perspective argue for a rejection of this notion, on the basis of a series of philosophical incompatibilities, Scheurich (1996) suggests otherwise. For Scheurich (1996) the creation of "wildly different kinds of validity" (p. 49) by parallel researchers are merely masks that conceal an overwhelming similarity in approaches to validity. That is, irrespective of the language ${ }^{45}$ used to identify the backdrop against which a piece of research is to be considered valuable, considerations of value ultimately involve a process - or the movement along a spectrum - for discriminating

as this would imply the production of trivial knowledge that repeats what is already known. Rather, if the knowledge claim conflicts with what is known, then it is for Hammersley (2009a) worth pursuing. He suggests, "While plausibility may lead to acceptance, implausibility does not lead to rejection, only to a demand for further evidence. In the end, this evidence may force us to revise some of the prior assumptions in terms of which the knowledge claim was initially judged implausible" (pp. 16-17). ${ }^{44}$ What is perhaps best described as a 'debate within the debate' is a series of claims and counter claims concerning an adherence to or rejection of a neorealist stance within qualitative criteria. On one side of the argument is Hammersley (1998, 2005, 2008, 2009a, 2009b), while on the other is Hodkinson (2004), Smith (1997), Smith and Deemer (2000) and Smith and Hodkinson (2005, 2009). Hammersley's (1998, 2005, 2009a, 2009b) argument culminates in a belief that the relativist position propounded by Smith and others is founded upon a number of assumptions about realism and the existence of things independent of a person's claims about them. In opposition to this, Smith and Hodkinson (2009) suggest we must accept a relativism on the basis of the finite nature of human understanding. A single footnote is unable to do justice to the depth of these arguments. For a more considered explication see: (i) Hammersley, (2009a) Challenging Relativism: The Problem of Assessment Criteria', and the response from, (ii) Smith, J. \& Hodkinson (2009) Challenging neorealism: a response to Hammersley. A final word can be seen from Hammersley who suggests Smith is 'closing down the conversation', a tactic employed within the title of a earlier article by Smith (1984), (iii) Hammersley (2009b) 'Closing down the conversation? A reply to Smith and Hodkinson'. ${ }^{45}$ On the topic of simply introducing 'new terminology' to the criteria argument, Smith (1997) describes this terminology as inescapably "parasitic on the old" (p. 8). He suggests that new terminology cannot begin to originate without recourse to its derivation. As such, the meaning of the terminology from which the new term derived is carried forward. Illustrative of this trend toward parasitic terminology can be seen in what Altheide and Johnson (1994) describe as "radically qualified" or "hyphenated" (p. 488) notions of validity. Several authors whose work subscribes to the mission statement of the Parallel perspective have sought to create a diverse range of prefixes for validity: descriptive validity, interpretive validity, theoretical validity (Johnson, 1997); transactional validity, transformational validity (Cho \& Trent, 2006); imperial validity (Scheurich, 1996), successor validity, situated validity, rhizomatic validity and voluptuous validity (Lather, 1993). Scheurich (1997), conferring with Smith (1997), believes the cultural baggage and paradigmatic heritage encompassed by the word validity makes its use a questionable activity. 
between research that one believes is good and research that is not (Scheurich, 1995, 1996, 1997). ${ }^{46}$

The parallel perspective seeks to provide a philosophically cogent argument for the adoption of alternative criteria. More specifically, these criteria would be alternatives to the positivist presuppositions held by the replicative position. However, the tautological nature of the parallel perspectives argument do little more than add what Schwartz-Shea (2006) describes as a "dizzying array of terminology" (p. 97) to the existing complexities of validity in qualitative research. Labeling themselves post-positivist in an effort to 'shake the positivist monkey' from their backs, scholars within the parallel perspective merely perpetuate an unconsidered theoretical falsehood. This view is supported by Sparkes (2001) who argues that "the trend for those working within its [the parallel perspective] confines has been to develop criteria that are closely related to those used by quantitative researchers, which are then simply fitted to a qualitative context" (p. 541). Such a manoeuvre does little to overcome a subscription to positivist presuppositions for making determinations of what matters or what is valuable in scholarly research. In other words, the argument upon which the parallel perspective is founded is little more than a tautological falsehood.

This inability to sustain a challenge against the replicative perspective raises the spectre of doubt as to which end of the relativism and neorealist spectrum parallel scholars are situated. Sparkes and Smith (2009) suggest that despite the philosophically untenable nature of the parallel perspective it remains the most preferred option, "even though the reality that is supposed to 'kick in' and adjudicate between trustworthy and untrustworthy interpretations has yet to do so in any published work to date" (p. 493). This thesis acknowledges that the parallel perspective goes to great lengths to suggest a number of philosophical inconsistencies between qualitative research and the replicative perspective. However, the assumptions that inform this argument ultimately adopt and therefore perpetuate a neorealist position. In fact, the arguments that underpin the parallel perspective represent a tautological vice. Regardless of the label that is substituted, the principal concern remains the discrimination between research that is good and research that is not. Although it

\footnotetext{
${ }^{46}$ Mishler (1990) and Lather (1986), both noteworthy proponents of the parallel perspective, provide an example of this slippage toward a tautological argument. Mishler (1990) believes that scientific judgement criteria are socially constructed and appropriated by a community of scientists as 'in-house' mechanisms for judging research as acceptable and research that is not. Mishler (1990) adopts the Kuhnian concept of exemplars and a community of scholars as a source for making discriminations about research as being above or below the line on the map. Likewise, Lather (1993) contends that validity is an insufficient consideration of qualitative research outcomes and rather believes the emancipatory aspects of a study should be the guiding consideration. Thus, here a researcher is seeking to make discriminations between research that is good at emancipating and that which is not good at emancipating; a tautology indeed.
} 
could be argued that scholars within the parallel perspective simply wish to 'hedge their bets' in the representation of a real, there is a growing body of work that is unapologetic about ignoring a concern for the representation of a real at all. Scholars accepting the Letting-Go perspective accept what they believe to be the inherent relativism that can be found within qualitative research. Those operating from this position call for the discipline to let-go of the notion of criteria all together.

\section{Letting-Go Perspective}

It is the words of Smith and Hodkinson (2005) that capture most adequately the argument upon which the letting-go perspective is based, "we have come to the end of our attempts to secure an epistemological foundation for our knowledge and must acknowledge that we are in the era of relativism" (p. 915). Scholars subscribing to the theoretical tenets of this domain advocate the abandonment or 'letting-go' of validity criteria on the basis of the irreconcilable philosophical differences between neorealist and relativist thought (Smith \& Deemer, 2000; Smith \& Hodkinson, 2005, 2009; Talburt, 2004; Wolcott, 1994). On the basis of these arguments, adherents of the letting-go position reach toward what are commonly referred to as alternative positions from which to judge their work (Angen, 2000; Barbour, 2001; Eisner, 2003; Fuchs, 1993; Whittemore, Chase \& Mandle, 2001; Talburt, 2004; Wolcott, 1990, 1994, 2005).

Smith and Hodkinson (2009) and Smith and Deemer (2000) take the view that the ontological relativism that underpins the qualitative paradigm and the subsequent appeal to a multiplicity of realities are incompatible with any epistemological foundationalist approach that seeks to 'tie-down' reality to that which is valid. So much so, that they go on to suggest that any such approach poses an untenable philosophical hypocrisy (Smith, 1990, 1993). Talburt (2004) shares this scepticism by emphasising what she describes as an underlying preoccupation to verify that the representation that one produces is indeed real. Talburt (2004) concedes that qualitative research does bump up against real events and real lives. However, she argues that representing these positions is a process of "constructing others' constructions of the constructed" (p. 81), or, to use the words of Geertz (1973), "explicating explications, or winks upon winks upon winks" (p. 9). For Talburt (2004) then, "these winks, either the 'original' or researchers' descriptions and interpretations of them, are never fully representable in language or verifiable as faithful copies of a real" (p. 81). Talburt (2004) goes on to echo Wolcott's (1990) contention that the quest to represent a real, and indeed the veracity of this real, lures qualitative researchers from the purpose of "opening new pathways for thought" (p. 81) in pursuit of what Lather (1993) describes as "a less comfortable social science" (p. 673). Thus, in order to ensure an adherence to these anti-foundationalist 
arguments, the letting-go perspective rejects the appropriateness of criteria. This thesis, however, contends otherwise.

Those within the letting-go perspective advocate the application of more 'emotive or moral criteria' as determinant of the value of research. In essence, this appeal is little more that another subscription to a 'neo-parallel' perspective. For example, Ellis $(1997,1999)$ acknowledges the criterion of evocation and asks the reader to consider the way in which the written text evokes a sense of the real story and creates emotions that makes the reader reflect on his or her own life. For Richardson (2000) the key criterion of impactfulness is a consideration of the contribution a piece of research has made to the reader. On the other hand, for Lincoln (1993) the criterion of success in qualitative work is its authenticity where the reader is invited into the text to experience the life being described. Similarly, Blumenfield-Jones (1995) considers believability and fidelity or the link between the life being represented and its representation to be the hallmarks of quality most specifically in autoethnographic work. While for Yardley (2000):

The decisive criterion by which a piece of research must be judged is, arguably, its impact and utility. It is not sufficient to develop sensitive, thorough and plausible analysis, if the ideas propounded by the researcher have no influence on the beliefs or actions of anyone else. (p. 223)

This myriad of moral criteria against which to determine the value of qualitative research provides what Sparkes and Smith (2009) describe as a pool of possible criteria from which to select in making determinations of value. ${ }^{47}$

The notion that one merely selects criteria from a broader 'pool' of possibilities represents for this thesis the hallmarks of a slippage into the theoretical anarchy feared by Silverman (2000). Moreover, it operates to highlight a lack of concern for the degree to which a representation bumps into the experiences of real people and real events. Instead, the concern here is for the production of little more that a good read. ${ }^{48}$ However, this thesis argues that making determinations of the value of a piece of work in terms of evocation, fidelity or its impactfulness is ultimately to make judgements about the value of the research against some form of criterion. Such criteria ultimately afford one an opportunity to discern

\footnotetext{
${ }^{47}$ In practical terms Sparkes and Smith (2009) suggest that the qualitative researcher develops a list of selected criteria. This list is not generated prior to research engagement itself, but rather while engaged in the study itself "where the list gets challenged and changed not by abstracted discussions but by the application and engagement with actual inquiries" (p. 495).

${ }^{48}$ Sparkes (2001), Smith (1997), Smith and Deemer (2000) and Smith and Hodkinson (2009) believe the association of the 'letting-go perspective' with the domain of relativism is misguided.
} 
research that is good from research that is not, drawing one all the more to question exactly what it is that these scholars are actually 'letting-go'.

Jones (2005), Sparkes (2002, 2003a, 2003b), and Tsang (2000) suggest the discipline look towards 'auto-ethnography' for an example of research that successfully employs the use of 'other' criteria to determine the value of research. ${ }^{49}$ However Atkinson and Delamont (2006), in an article titled 'Rescuing narrative from qualitative research', argue that qualitative researchers are quick to champion uncritical narrative accounts. These authors go on to argue that these uncritical practices - which are common in auto-ethnographic accounts - lead to unreflective use of personal accounts. In fact, this uncritical subscription to persuasive writing leads Coffey (1999) to challenge auto-ethnographical work's place as a subset of ethnography proper.

Some would say that such texts are not "doing" ethnography at all, but are self-indulgent writings published under the guise of social research and ethnography... it remains debatable as to whether utilizing ethnographic strategies to write autobiography really "counts" as ethnography at all. (pp. 115-116)

Having considered the movements made within auto-ethnographic work and a call for a recognition of the artistic and poetic representations produced, this author cannot avoid being brought to recall other brilliant stories. Stories where people come to life for the reader and, to use the terms that are common to the letting-go perspective, have a sense of fidelity and contextual sensitivity. However, these stories are fictitious, stories like The Hobbit and The Lord of the Rings for instance, each a journey into the historically nuanced and situated worlds of people's lives. These tales, just like those of auto-ethnography, are able to meet the creative or poetic criteria for judgment as proposed by the letting-go perspective. However, in the absence of a series of standardised criteria for assessing the validity or the degree to which the qualia of real people are represented within a piece of qualitative research, readers are unable to discern genuine representation from simply a good read. For that matter, without standard criteria we are unable to discriminate between what is real and what is merely fiction. This disregard for the representation of a real inherent in the letting-go perspective, operates to blur the - already fuzzy - line between relativism and fiction (Hammersley, 2009a). ${ }^{50}$ If the only means of discernment with regard to a piece of research

\footnotetext{
${ }^{49}$ For some preliminary work concerning this issue see: Garratt and Hodkinson (1998) 'Can there be criteria for selecting research criteria? A hermeneutical analysis of an inescapable dilemma'.

${ }^{50}$ This thesis takes the view that the subscription to relativism is a surreptitious recognition that at some level contemporary approaches to qualitative research are incapable of laying claim to
} 
is that it 'sounds reasonable' to the reader, then the qualitative paradigm is at risk of becoming nothing more than a genre within the fiction section of the book store and university libraries.

It has been suggested here that due to the perpetuation of a naïve assumption about the nature of language and meaning that is necessitated by the closing down of the dialogic opportunity, qualitative research cannot claim to represent in any valid way the qualia of the real people and real lives bumped into in research. In line with this, it is suggested that proponents of the letting-go perspective not only openly accept this claim, but in fact that this claim underpins the use of evocative or poetic criteria. In other words, scholars within the letting-go perspective acknowledge the inability of current practices of qualitative research to represent a real and instead reach toward criteria that offer some degree of value to their work. However, if the arguments that are advanced by the letting-go perspective were accepted as given, then the claim to legitimacy of all qualitative research outcomes would be rightly open to challenge and speculation. In fact this thesis suggests that in the absence of any consideration of validity we have no means of discerning even the possibility that those experiences of real people that the researcher bumps up against are actually being represented. To follow this line of thought is to give up on the possibility that qualitative research can legitimately represent real people and real lives at all.

In bringing this discussion of the ever circular and monotonous criteria debate $\mathrm{e}^{51}$ to a conclusion here, what it does is highlight a widespread ambivalence within the discipline concerning the representation of a real. This ambivalence highlights a pervasive neglect for the way in which real people and real lives are represented in qualitative research. In turn,

representing a real. In fact, it is suggested here that the development of a post-facto series of criteria upon which to celebrate mediocrity in the stakes of representing a real is as good as it is going to get. ${ }^{51}$ The circularity of this debate can be seen in the following arguments. Sparkes (2001) captured the entire criteria debate with his statement "Of course what counts, brings us back full circle to what criteria are used to judge what counts in the first place" (p. 48). Similarly Elliot, Fischer and Rennie (1999) hold that the value of any scientific method must be evaluated by its ability to provide meaningful answers to the questions that inform the research. These two summations highlight the complexity of the overall debate and work as a vehicle for the ever-circular or monotonous tail-chasing dialogue that is associated with the notion of validity criteria in qualitative research. Despite this view, Wolcott (1995) suggests, the issue of validity in qualitative research may be dismissed as consuming research energy, but it is an issue that will not go away. Supporting this position, Angen (2000) believes the pursuit of qualitative criteria of validity will persist for as long as the positivist approach to research is considered the 'gold standard' of the academy. In other words, while "the desire for legitimacy in an academic world that is still tied to positivism keeps interpretative researchers questing for a recipe or map that will legitimise their efforts" (p. 379), questions of validity will persist. However, for this thesis the adherence to naïve assumptions about language and meaning as well as a pervasive lack of concern for representations of a real - as evidenced by the Criteria Debate - cast doubt upon the capacity of qualitative research to represent the experiences of real people faithfully as a real. 
this discussion also emphasises the uncritical perpetuation of research practices that are founded upon a naïve account of language and meaning that ultimately undermine the representation of a real by contemporary qualitative research even further. However, despite the supposedly incommensurate differences that characterise each the three threads of the criteria debate, it has been suggested here that each thread ultimately proposes a series of criteria with which to make discriminations between research that is good and research that is not. In other words, each perspective of the debate at some level represents an effort to tiedown what is valuable about a piece of research.

According to the parallel and letting-go perspectives, we as researchers are free it seems to choose the criteria with which a piece of research will be considered valuable. In light of this, this thesis chooses the criteria of validity. Not validity in terms of the real, rather validity in terms of a real. This seemingly subtle difference is highly significant. The interpretive nature of all human understanding means that it is not possible for us to achieve understanding at the level of the real. On the other hand, understanding at the level of a real, as an understanding of the way that something is for an individual person, is indeed a human possibility. Thus, in acknowledging validity in terms of a spectrum and not as a point of perfection, it is possible to represent real lives and real people in a way that is valid. What is meant by this conceptualisation of validity is embodied by the words of Arendt (1958) who suggests that "even if there is no truth, man can be truthful, and even if there is no reliable certainty man can be reliable" (p. 279). This thesis therefore takes the view that by getting as close as possible to the phenomena of interest and by representing that phenomena - the idiosyncratic qualia of an individual person - as faithfully as possible, the resultant representation will lay claim to validity and to the representation of a real.

Although proponents of the qualitative research discipline might seek to suggest that this final sentence is indeed an accurate portrayal of the current practice within qualitative research, the preceding discussion clearly suggests otherwise. In light of this, it is toward the development of a new theoretical approach for qualitative research that this thesis now turns. This is a turn toward an approach that advances the centrality of the dialogic in understanding, and in so doing provides an alternative to the naïve assumptions about language and meaning that are inherent in traditional approaches to qualitative research.

\section{The Individual as the Focus of the Qualitative Endeavour}

In reaching toward the development of a new theoretical position that not only provides a redress to the representational challenges identified within contemporary approaches to qualitative research, but also provides a foundation for apprehending the qualia 
of an individual person, a return to the initial question posed by this thesis is a useful touchstone.

Is it possible that qualitative research has contributed less than would seem attainable to the study of individuals because we as qualitative researchers have been working on the basis of a number of assumptions about people, which we have not examined very carefully, and have also adopted a number of assumptions about the nature of science which are less than adequate for the subject matter we have chosen?

The preceding discussion highlighted that the naïve assumptions about language and meaning that are inherent within contemporary practices within qualitative research, opens up to challenge any claim to the representation of the real people encountered or the representation of a real. It has been argued that approaches that adopt a naïve account of language as a vehicle that merely and unambiguously points at things, is only capable of providing at best a superficial level of abstraction of the individual people involved. Moreover, the concern for the search for generalisation which permeates all qualitative research, as evidenced by the ubiquitous nature of thematic representation, operates to 'zoom out' from the particular to the glaringly obvious.

On the basis of these assumptions it is argued that less than what is considered to be possible by contemporary qualitative research has been contributed to the focused study of the person. Given that Denzin and Lincoln (2005) suggest that at the core of qualitative research is a compulsion to "describe routine and problematic moments in individuals' lives" (p. 3, emphasis added), the paucity of depth in the understanding of the individual person that we have available today is arguably an indictment upon the discipline of qualitative research more broadly. ${ }^{52}$ It is for this reason that this thesis holds most hope for an approach that makes the focused study of the individual person its core concern. It is argued that a focus upon the individual person will provide a sound redress for the theoretical challenges illuminated here. Furthermore, it will provide an opportunity to achieve a greater depth of understanding, or a higher level of abstraction of the real people encountered in qualitative research. Given that many of the issues concerning validity in qualitative research arise when comparing the experiences of one person with those of another, focusing upon the individual

\footnotetext{
${ }^{52}$ The Handbook of Qualitative Research (Denzin \& Lincoln, 2005) is considered the 'gold standard' within the discipline of qualitative research. However, this claim to the superiority of the text is challenged by Brinkmann (2007) who, when describing the apparent ignorance of Freud's work within qualitative research, states "it is noteworthy that the authoritative Handbook of Qualitative Research (Denzin \& Lincoln, 2005), contains very few references to those leading psychologists who have influenced the field of qualitative inquiry" (p. 1118).
} 
person, rather than a group of seemingly homogenous individuals, will support the development of valid representations. As such, this thesis moves into the domain of an approach where the focused study of the individual sits comfortably - idiographic science.

\section{Idiographic Introductions}

The philosophical and theoretical tenets of the idiographic perspective with which this thesis is most centrally concerned will be elaborated further in the next chapter. However, in preface to this turn, at the core of the idiographic position is a focus upon the 'within person' elements of the individual person. An understanding at this level of abstraction is beyond the subordination to any subsequent level of generality (cf. Barlow \& Nock, 2009; Nesselrode, Gestorf, Hardy \& Ram, 2007; Smith, Harre \& van Langenhove, 1995, 1996). ${ }^{53}$ More recently, Molenaar (2004) and Nesselrode et.al. (2007) suggest that an idiographic perspective can return to scientific thought a dedicated study of the individual prior to any search for generality across individuals, and in so doing reinstate the individual as the rightful unit of analysis for psychology. This view is shared by Smith, Harre, and van Langenhove (1996) who emphasise a pressing need for idiographic understanding.

Given the neglect of the idiographic level of inquiry and the consequent paucity of focused understanding of psychological functioning of the individual, there is some urgency in the need for projects that take as their task the detailed description of individual human beings. (p. 63)

Thus the intention here is to develop a theoretical approach that enables one to zoom in on these within person elements - or qualia - for the purpose of qualitative research. This intention is not only timely but is comfortably situated within the idiographic approach.

\section{Chapter Summary}

This chapter has established that on the basis of a pervasive, adherence to a naïve assumption about language and meaning, the discipline of qualitative research is unable to legitimately provide representations of the real people and real lives encountered. Moreover, the ambivalence that surrounds the value of a real - as embodied by the Criteria Debate further highlights the representational crisis that plagues the discipline. As a consequence of the perpetuation of these assumptions, it is argued that qualitative research has contributed less to the study of the person than could have been possible. In response it is the focused study of the individual person that will provide an opportunity to represent more deeply the

\footnotetext{
${ }^{53}$ It is the search for generalisable laws that dominates scientific thought. A position embodied by the term nomothetic perspective which is the direct antithesis to the idiographic perspective.
} 
'within person' elements of the people encountered. Thus a turning toward the idiographic perspective "as if",54 (Vaihinger, 1925/1952) is an entirely appropriate avenue to pursue in overcoming these challenges, as well as providing a deeper understanding of the experiences of real people. This avenue is embodied in principle by the work of George Kelly and his Personal Construct Psychology.

\footnotetext{
${ }^{54}$ In his text The Philosophy of As-If, Vaihinger (1911/1952) suggested that at times in the movement of scientific thought one should not ask whether something is true in a non-pragmatist sense (this cannot be discovered), but whether it is useful to act 'as if' it were true. Religious and metaphysical doctrines provide useful examples here.
} 


\section{CHAPTER 3}

\section{THE IDIOGRAPHIC PERSPECTIVE}

The previous chapter established that contemporary approaches to qualitative research are founded upon a naïve assumption about language and meaning. This assumption challenges the degree to which such approaches can be considered to represent $a$ real. Reaching toward the idiographic perspective 'as if' it is an entirely appropriate avenue to pursue in overcoming these representational challenges, this chapter will provide a considered exploration of the theoretical principles of the idiographic position. Embodying - in principle - the tenets of this idiographic position George Kelly's The Psychology of Personal Constructs is considered. As well as unpacking the theoretical principles of Kelly's position, this chapter begins to emphasise the implications of the way in which Kelly dealt poorly with language. The chapter culminates in an acceptance of Kelly's invitation for the theoretical elaboration of his work and suggests that the direction of language is the avenue to pursue.

\section{Thinking Idiographically}

Idiographic science begins from the premise that each individual is unique in all respects - genetically, physiologically and psychologically - and ultimately experiences life in idiosyncratic ways (Molenaar \& Valsiner, 2008). Given that for many scholars there cannot be science without generalisation, Mininni (2008) suggests that idiographic science is often considered a "contradictio in terminis" (p. 251), as 'a contradiction in terms'. Despite the 'stronghold' of nomothetic science and its subsequent approach to understanding the human subject, scholars within the domain of idiographic science propound a compelling argument that challenges core nomothetic assumptions. These thinkers argue that the nomothetic notion of generalisation represents a scientific inadequacy in understanding human beings, and as such believe traditional nomothetic principles do little to support a univocal concept of science (Lamiell, 2003; Mininni, 2008; Molenaar, Huizinga \& Nesselrode, 2003; Molenaar \& Valsiner, 2008; Valsiner \& Sato, 2006). At the core of this challenge is the assumed homogenisation of social groups that underpins the making of generalisations from samples to populations. The following passage from Molenaar and 
Valsiner (2008) captures the theoretical challenges that are inherent in the nomothetic approach to human understanding.

Such discourse treats all members of the class as if they formed a crisp (rather than fuzzy) set, glancing over the obvious intra-class differences as if these are theoretically unimportant. Indeed, when a socially mono-functional role - such as soldier's - is expected to act as a collective unit, the issue of inter-soldier differences is socially irrelevant. Yet social irrelevance is not equal to theoretical irrelevance. Psychology has failed to clearly see that and its tradition has become to find general laws that hold equally for all subjects in some homogenous population. (p. 24)

For Molenaar and Valsiner (2008), the application of statistical procedures is ultimately designed to reduce natural invariance between participants and is beholden to an assumed homogenisation of the qualitative aspects of being human. ${ }^{1}$ These authors go on to note that although the production of such 'assumption laden' information may be of use for funding agencies and have wider journalistic appeal, it is of little use in the building of knowledge of the basic psychological functioning of particular persons (Jones, 1971; Molenaar, 2004; Molenaar \& Valsiner, 2008; Valsiner, 2004). ${ }^{2}$ Support for this argument can also be found in the work of Nesselroade, Gerstorf, Hardy and Ram (2007) who suggest that, despite the acceptance of the idiosyncratic position as fundamentally important for psychological investigation, the current practice of diluting idiosyncrasy by averaging it out across cases is neither satisfying nor ultimately productive. ${ }^{3}$

\footnotetext{
${ }^{1}$ The synergies between the challenges being levelled by this thesis at contemporary qualitative research practices and those levelled at this uncritical adherence to nomothetic conceptions of science are palpable.

${ }^{2}$ Molenaar and Valsiner (2008) go on to develop Molenaar's (2004) earlier work on the application of systematic analysis of single case studies over time. They suggest that the case study when examined in such a way provides the primary empirical objects for arriving at generalised knowledge.

${ }^{3}$ It was with some surprise that the work of Allport warrants only one reference in dispatch within the

'Yearbook of Idiographic Science (2008)'. In light of this, a most opportune time has arrived to mention the work of the prolific psychological scientist Gordon Allport. It is Allport who is arguably responsible for introducing the idiographic approach into American psychological science. It is claimed that Allport borrowed the terms idiographic and nomothetic from the German philosopher Wilhelm Windelband. However, Hurlburt and Knapp (2006) argued that it was in fact Hugo Munsterberg who introduced these terms into psychology in 1898. Conjecture aside, Allport (1962) was dissatisfied with the outcomes of statistical approaches and the neglect of the perspective of the individual as being "like no other man [sic]" (p. 410). Here he argued that the unique biography of any one individual involved in a study could not be recovered from the final research outcomes. Thus, he concluded his argument by calling for the idiographic approach to be recognised as a legitimate pursuit within psychological understanding. The following quote by Allport (1962) is explanatory of this dissatisfaction and indirectly points towards what Allport believed to the productive possibilities of the idiographic perspective. "Suppose we take John, a lad of 12 years, and suppose his family background
} 
The longstanding nature of this argument can be traced through the work of Lamiell $\left(1981,1987,1997,1998,2003,2007,2009\right.$ a, 2010), a prolific contributor to the genealogy ${ }^{4}$ and theoretical development of idiographic science more generally. Lamiell (1987, 2003, 2010) extends the argument outlined above and contends that research adopting a 'social homogenisation' approach cannot satisfactorily be considered as either 'nomothetic' or 'idiographic' on the basis of the following argument. Individuals have certain properties that make them unique and if any theory is to be capable of making general laws it must be capable - as a minimum - of effectively representing these unique properties. Lamiell's (1987) argument is captured most succinctly in the following passage.

The empirical findings generated by individual differences research cannot be interpreted at the level of the individual and consequently cannot possibly inform in an incisive manner a theory of individual behaviour/psychological functioning... That is, the empirical findings generated by such research cannot logically establish that something is the case for each of many individuals. (pp. 90-91)

More recently, Lamiell (2010), championing the merits of an idiographic understanding, suggests that a conceptualisation of the person as entity is what is required to further a genuine understanding of the person within the discipline of the human sciences.

With increased appreciation for the conceptual confusion that is always and necessarily embedded in any attempt to regard the aggregate statistical findings issuing from correlational studies of individual differences as interpretable in any way at all for individuals, the need for a coherent conception of persons as entities quite distinct from person variables will become ever more apparent. (p. 141)

is poor; his father was a criminal; his mother rejected him; his neighbourhood is marginal. Suppose that 70 per cent of the boys having a similar background became criminals. Does this means that John himself has a 70 per cent chance of delinquency? Not at all. His unique world contains influences unknown to the statistician: perhaps an affectionate relation with a certain teacher, or a wise word once spoken by a neighbour. Such factors may be decisive and may offset all average probabilities. There is no 70 per cent chance about John. He either will or will not become delinquent. Only a complete understanding of his personality, of his present and future circumstances, will give us a basis for sure prediction" (pp. 411-412). It must be made clear here that although Allport was an advocate for statistical reasoning, he was cautious not to allow himself to be 'caught up' by the provocative generality that the uncritical use of statistical practices can lead towards.

${ }^{4}$ Lamiell has contributed significantly to the re-introduction of the work of William Stern and the notion of personalism back into modern psychological consideration. A great example of this can be found in: Lamiell (2009b) Psychology and personalism by William Stern. 
Implicit within these arguments, and those being established by this thesis, is that the inherently unique and deeper qualities of an individual are not recoverable from, or are rather lost, to the group averages or processes of social homogenisation. While being careful not to downgrade nomoethetic statistical approaches entirely, Proctor (2011) suggests that the uncritical use of these methods risks the production of findings "which are not true of any individual situation in particular, the danger being that the latter is lost amidst evidence attached to the group that the individual appears to belong to" (p. 33). As emphasised in the previous chapter, this process of social homogenisation is at the core of thematic representation and is what ultimately underpins contemporary qualitative practice. Founded upon a series of assumptions concerning the person as a homogenous being, this loss of individual qualities is embodied in what Kastenbaum (1985) describes as "indeterministic statistical zones that construct people who never were and never could be" (p. 7). Here the unique individual is indeterminate within and is not represented by the statistical representation that is produced.

In thinking idiographically we lay claim to dealing with the individual person(s) who are intrinsically unique, singular and deeply understandable (Salvatore, Tebaldi \& Poti, 2008; Salvatore \& Valsiner, 2008). However, it is the words of Mininni (2008) that embody the fundamental element of idiographic science for this thesis, "Personal experience is worth to be accurately described not although it should be regarded as 'unique' or 'particular', but quite because it is so" (p. 255). Here Mininni (2008) emphasises that idiographic science is more than merely another perspective from which to envision the human being. It is recognition of the nature of being human itself. As such, the theoretical positioning embodied by Mininni's (2008) words locates the idiographic perspective as a suitable theoretical position within which to situate the development of the theoretical position that this thesis is proposing.

The previous discussion has established that it is entirely appropriate for an interested observer to make the focused study of the individual person a satisfactory and worthy pursuit. That dimension of the idiographic position that this thesis is specifically concerned with is what Kelly (1955) refers to as the "inward outlook" as opposed to an "outward inlook" (p. 183). ${ }^{5}$ Providing a useful conceptualisation of the directionality of understanding, the 'outward inlook', akin to looking in to something from the outside, represents the perspective

\footnotetext{
${ }^{5}$ The use of this terminology has its origins within the work of Kelly (1963) who states: "The Psychology of Personal Constructs is a disciplined psychology of the inner outlook... an unabashed alternative to the scientistic psychologies of the outer inlook, and... a calculated step beyond the experiential psychologies of inner inner feelings" (p. 183). Kelly's use of pragmatic language makes for a 'user-friendly' theoretical elaboration and is adopted here in anticipation of doing the same.
} 
of an observer - in this case a researcher - casting a gaze upon another person in order to develop an understanding. It is this notion of an 'outward inlook' that represents the issue with contemporary qualitative research. In order to develop an understanding of another person's experiences, after the dialogical opportunity of the research interview has passed, the researcher is obligated to adopt a naïve view of language and meaning. Here the researcher must 'look into' the transcripts of the participant and make judgements about and extract those aspects of another person's life that are central to or have meaning for that person. However, as has been discussed previously, these judgments compromise the representation of the experiences of real people and instead signify a shift away from the actuality of the subject matter to be represented. In contrast, this thesis - borrowing from Kelly's (1955) 'inward outlook' - locates the inner outlook as those inner dimensions of being that a person can bring to language and therefore a dialogue. ${ }^{6}$ Thus, this inner outlook embodies a view that a person's within elements of being can indeed be shared through outward dialogue. A closer examination of Kelly's position will emphasise what is meant by this inner outlook.

\section{The Psychology of Personal Constructs}

A theoretical approach that is sufficiently commensurate with the 'inner' aspects proposed by the idiographic position and also provides an appropriate 'starting point' for the theoretical developments being proposed by this thesis, is the major work of George Alexander Kelly, titled The Psychology of Personal Constructs. ${ }^{7}$ Before commencing a considered elaboration of his theoretical position, it must be stated from the outset that the Psychology of Personal Constructs is, by itself, unable to meet the task of representing the inner outlook of the individual person envisaged by this thesis. This is due to what is argued to be an insufficient grounding in the languaged aspects of being human. In fact, Kelly (1955) does "not appear to have adequately dealt with the topic of language" (Chiari \& Nuzzo, 2004, p. 57). ${ }^{8}$ Evidence of Kelly's inadequate assumptions about language can be seen clearly in his own words and provides a preface for the discussion to follow. Here Kelly (1958) emphasises the separation between the construct and language, a separation that will

\footnotetext{
${ }^{6}$ The terms 'inner outlook' has been adopted here over 'inward outlook' as propounded by Kelly (1955). The term 'inward' carries a connotation that the person is 'looking in' at something. However, this thesis is moving toward a conceptualisation of the inextricability of language and a person's innermost processes of understanding. In such a conception there is not anything more inward than the language of the inner itself. That is, it is language that is the essential condition of this inner. From here on the term inner outlook will therefore be used for elaborating Hermeneutic Constructivism. ${ }^{7}$ Chiari and Nuzzo (2003) describe Kelly's Personal Construct Psychology as "so much ahead of its time that his work is only now at the cutting edge of contemporary psychology and psychotherapy" (p. 42).

${ }^{8}$ The implications of the way in which Kelly (1955) dealt poorly with language and more specifically the way in which he located the construct outside of language are the focus of much to come in this thesis.
} 
be shown, in the following chapter, to challenge the overall theoretical tenability of his position, "The personal construct we talk about bears no relation to grammatical structure, syntax, words, language, or even communication... it is simply a psychologically constructed unit for understanding human processes" (p. 87).

As will be shown in both this and the following chapter, the Psychology of Personal Constructs is open to the challenge of succumbing to a naïve assumption about language and meaning that ultimately challenges the theoretical sustainability of the position itself. The unsophisticated account of language that can be seen within Kelly's (1955) work is incommensurate with the theoretical unpacking of the inner outlook that is being developed by this thesis for the purposes of qualitative research. Despite this, an introduction to Kelly's (1955) work provides a worthy entree to a further consideration of this argument. A sketch of some of the general theoretical and philosophical elements of the Psychology of Personal Constructs, and their compatibility with the representation of the more 'inner' dimensions of the idiographic perspective will therefore be provided. This sketch will include some preliminary commentary on the way in which Kelly (1955) locates the construct beyond the reach of language. In so doing, it will provide the foundation for a closer examination of the implications of this upon the tenability of Kelly's Personal Construct Psychology and its subsequent potential as a theoretical foundation for the position being developed for this thesis. This discussion will be pursued in the following chapter.

\section{More 'Inner' than 'Outer': An Idiographic Introduction.}

With more than thirty years of experience with both theoretical and practice based elements of clinical psychology, ${ }^{9}$ Kelly (1955) sought to develop a psychology that offered a reconsideration of many of assumptions that informed the psychological approaches contemporary to his time. Ultimately Kelly (1955) wanted to "give life back to the person who lives it" (p. 37). In so doing, he sought to provide a more humanistic alternative that he believed would not deprive humans of their capacity to make decisions and be in charge of their own lives (Holland, Neimeyer, Currier \& Berman, 2007; Fransella \& Neimeyer, 2003). Kelly (1955), underpinned by a belief "that each man $[\text { sic }]^{10}$ contemplates in his own personal

\footnotetext{
${ }^{9}$ As well as undertaking some twelve years of clinical experience during the Great Depression within Kansas, Kelly - in conjunction with a series of students - developed and conducted a series of travelling psychological clinics to assess children in schools. Perhaps the most thorough and at times inspirational 'tellings' of Kelly's personal, professional and theoretical developments can be seen in: Fransella (1995). George Kelly, and Neimeyer and Jackson (1997). George.A.Kelly and the development of personal construct theory.

${ }^{10}$ This is an acknowledgement of the problematic use of the preposition him, as the normal or standard view, that can be seen throughout Kelly's work. Although inconsistent with the principles of gender
} 
way the stream of events upon which he finds himself so swiftly born" (p. 3), begins an elaboration of the Psychology of Personal Constructs. He draws his position to its philosophical height through the concept he terms Constructive Alternativism. ${ }^{11}$ Kelly (1955) elaborates this as follows:

To the living creature, then, the universe is real, but it is not inexorable unless he chooses to construe it that way...We assume that all of our present interpretations of the universe are subject to revision or replacement. We take the stand that there are always some alternative constructions available to choose among in dealing with the world. No one needs to paint himself into a corner; no one needs to be completely hemmed in by circumstances; no one needs to be the victim of his biography. We call this Constructive Alternativism. (pp. 8, 15, emphasis in original)

For Kelly (1955), Constructive Alternativism represents the philosophical roots that underpin the Psychology of Personal Constructs and the fundamental tenets from which his broader theoretical position springs forth. Kelly (1955) refers to Constructive Alternativism in terms of a "philosophical point of view" (p. 16), however he openly recoils from providing a "fullyfleshed out ${ }^{12}$ philosophical approach that addresses traditional problems within philosophy. ${ }^{13}$ It is interesting, however, that those aspects from which Kelly sought to distance himself the development of complete philosophical and scientific systems - are those same aspects that sustain much of the ongoing theoretical, clinical and research dialogue today. Warren (1998) offers what could be considered some justification for this quandary.

Personal construct psychology is an attempt to deal with the whole complex of meaning-giving. What is given is an account of human behaving which

neutral language, material quoted from Kelly's work will be presented in its original format, and no further reference to its archaic nature by way of [sic] will be provided.

${ }^{11}$ Kelly contrasts Constructive Alternativism with Accumulative Fragmentalism, this latter a position that holds that knowledge is merely an accumulation of facts - much like pieces of a jigsaw puzzle and that one day, the person who accumulates these facts will know or will have the answer. In contrast Constructive Alternativism supports a position whereby each event is interpreted in a manner that means it could be reinterpreted differently in the future (Kelly, 1955).

${ }^{12}$ In recoiling from the development of fully-fleshed out philosophical system, Kelly (1955) states that "while we have no intention of trying to build a complete system at this point, it does seem to be incumbent upon us to attempt to be explicit about some of our more important prior convictions" (p. 6).

${ }^{13}$ Warren (1998), recalling the work of Australian Philosopher John Anderson (1962), contends that the notion of a "systematic philosophy" over and above a "philosophical system" best captures the philosophical position exemplified by Constructive Alternativism (p. 49). As he describes it, a philosophical system represents a thoroughgoing account of all that exists, while a systematic philosophy represents a series of principles that apply to all phenomena. It is this latter framework that Warren (1997) considers to be implicit within the Constructive Alternativist position. 
comprehensively encapsulates the various aspects that psychologists and philosophers have tried to isolate: cognition, affect, volition. (p. 6)

Since its appearance in the mid-1950s the Psychology of Personal Constructs has received varying degrees of attention. Not only in terms of its stated focus on psychology, clinical psychology and personality, but also for the way in which Kelly addressed the philosophical 'situatedness' of his work. This was indeed a bold and uncommon practice amongst scholars at the time of Kelly's writing and publishing The Psychology of Personal Constructs. ${ }^{14}$ However, Kelly - steeped in the traditions of pragmatist thought ${ }^{15}$ - was more interested in getting on with the job of propounding his psychological theory than providing an exhaustive genealogical ${ }^{16}$ homage to his philosophical roots. ${ }^{17}$

Kelly's appeal to philosophical pragmatism (Butt, 2000a, 2008) can be seen frequently within his work. In fact, these pragmatic principles can be witnessed within the structure of his own writing. Kelly (1955) frequently establishes 'practical bridges' that allow him to elide possible theoretical confrontation, and provide an avenue by which to return pragmatically to the key focus of his consideration - the development of an applied psychology. The following is merely one example of such a 'practical bridge' that also provides a useful segue to a consideration of the idiographic position that can be 'read between many of the lines' of Kelly's work:

Scholars customarily distinguish between the forms of thought and the actual thinking behavior of people. The study of the former is classified under philosophy... while the latter is considered to be psychology. But we have

\footnotetext{
${ }^{14}$ The separation of the words writing and publishing here is not a simple oversight. Kelly produced a manuscript of his work but never expected any publisher to take an interest in his work and so delayed publication. However, under what Fransella and Dalton (2000) describe as "some unknown internal pressure" (p. 3), Kelly sent his manuscript to twenty publishers, six of whom agreed to publish his work.

${ }^{15}$ Kelly (1955) acknowledged his debt to the tradition of pragmatism and John Dewey. In doing so he highlighted his debt to the self imposed instrumentalist, as exemplified through the following: "Dewey, whose philosophy and psychology can be read between many of the lines of the psychology of personal constructs" (p. 154). The influences of the work of Dewey 'within the pages' of Kelly's work are discussed in the following three informative works: Warren (1998). Philosophical Dimensions of Personal Construct Psychology. As well as Warren (2003). 'Pragmatism and religion: Dewey's twin influences? A consideration of the links?' and Warren (2010). 'Kelly's personal construct psychology and Dewey's pragmatism: some direct and some 'intellectual context' aspects.

${ }^{16}$ Several authors have criticised Kelly for not sufficiently acknowledging his debt to the work of other thinkers, a lack they suggest might also explain his perhaps over-hasty rejection of specific domains of thought (Holland, 1970; Neimeyer, 1985). Most notably for this thesis the area of phenomenology is one that Kelly outwardly rejects. However, more recently Warren (1985) and Butt (1997a, 1997b, 2003) have suggested that Kelly misunderstood phenomenology. This discussion will be picked up in more detail later in this thesis.

${ }^{17}$ For a valuable discussion of these issues see: Warren, (1998). 'Philosophical Dimensions of Personal Construct Psychology.'
} 
taken the view that whatever is characteristic of thought is descriptive of the thinker; that the essentials of scientific curiosity must underlie human curiosity in general. If we examine a person's philosophy closely, we find ourselves staring at the person himself... what we are proposing is neither a conventional philosophy nor a conventional psychology. As a philosophy it is rooted in the psychological observation of man. As a psychology it is concerned with the philosophical outlooks of individual man (pp. 15-16)

The task with which Kelly was most concerned is clearly the development of a psychology at the level of the individual person. Warren (1998) supports this position, stating that, "Personal construct psychology was developed as a psychology of the individual, an idiographic psychology" (p. 72). ${ }^{18}$ Indeed, the philosophical position set forth by the notion of Constructive Alternativism is exemplified by Kelly's (1955) Fundamental Postulate ${ }^{19}$ - a theoretical position that informs the remainder of the Psychology of Personal Constructs which reads:

A person's processes are psychologically channelized by the ways in which he anticipates events. (p. 46)

In elaborating this postulate further, Kelly (1955) underscores the commensurate nature of the Psychology of Personal Constructs with an idiographic position: "Our first consideration is the individual person rather than any part of the person, any groups of persons, or any particular processes manifested in the person's behavior" (p. 47). Implicit within the fundamental postulate is the notion that each person creates for him or her self a series of unique psychological processes, which ultimately determine the way in which an individual person anticipates, makes sense of, and understands the world.

It follows therefore that if these self-determined anticipations dictate what is ultimately made of the world, and that the individual person creates these anticipatory psychological processes, then that same individual can indeed recreate them in order to

\footnotetext{
${ }^{18}$ This position is supported clearly by Harre (2006). While discussing the Psychology of Personal Constructs, Harre (2006) is clear that "the method was entirely idiographic, that is the research target was always the construct system of an individual person" (p. 67).

${ }^{19}$ Although the Fundamental Postulate will be elaborated later in this thesis, at this stage a definition of the term postulate is warranted. For Kelly (1955) a postulate is an assumption, "it is an assumption so basic that it antecedes everything which is said in the logical system which it supports" (pp. 46-47). A postulate, then, is not here a given truth, but rather the most basic assumption that forms the foundation and boundaries within which phenomena can be conceptualised and understood. If at any time a postulate is questioned, so too is the perspective provided by the postulate. In such a case the original postulate no longer provides the conceptual boundaries for understanding phenomena, being replaced instead with a subsequent postulate. Kelly wishes to highlight to the reader the ease with which we can slip into a different postulate and, no longer be using his postulate as the perspective for understanding.
} 
anticipate the world in different ways. ${ }^{20}$ This self-regulated adjustment in one's anticipations - a person's 'inner outlook' - is the hallmark of Kelly's notion of Constructive Alternativism and the embodiment of the idiographic principles within the Psychology of Personal Constructs more broadly.

One cannot help but be reminded here of the words of Mininni (2008) that exemplify the domain of idiographic thought with which this thesis is concerned and with which the Psychology of Personal Constructs is theoretically consistent, ${ }^{21}$ "Personal experience is worth to be accurately described not although it should be regarded as 'unique' or 'particular', but quite because it is so" (p. 255). Proctor (2011) suggests that Kelly's approach provides an opportunity to "look carefully at an individual's unique experience", and in so doing, "we can be more objectively faithful and detailed in our understanding of that individual's world and experience" (p. 34). Thus, the theoretical embodiment of 'unique' and 'particular' experience, as the mode of a person's being, can be traced through Kelly's Fundamental Postulate and the broader notion of Constructive Alternativism, squarely justifying a closer examination of this theoretical position.

\section{Theoretical Reflexivity}

The Psychology of Personal Constructs is sufficiently robust with idiographic principles and therefore commensurate with the conceptual framework to be developed by this thesis. However, the notion of theoretical 'reflexivity' as outlined by Kelly (1955) provides a valuable vehicle for determining which avenues to pursue in the unpacking of the theoretical development proposed by this thesis. Kelly (1955) suggested, for instance, that a psychological theory must "account for itself as a product of psychological processes" (p. 39), and perhaps more importantly that a psychological theory can and should be applicable to our own psychological processes.

As McWilliams (2004) so aptly states, "I believe that if I wish to consider a theory of personality as useful, I should be able to apply the theory to my own psychological processes and see myself reflected in the theory" (p. 291). Although the development of a theory of personality is not the task at hand, the tenets of McWilliams' (2004) notion of reflexivity are

\footnotetext{
${ }^{20}$ Kelly (1955) is keen to note that the adjustments in a person's anticipations and the subsequent change in his or her 'inner outlook' is not an 'ad hoc' process, such that, "Constructs cannot be tossed about willy-nilly without one getting into difficulty" (p. 15). The lawful nature that informs the adjustment of a person's constructions or anticipations will be explored in greater depth later this chapter.

${ }^{21}$ Supporting further a consideration of the Psychology of Personal Constructs as idiographic, Butt (1997a) suggests, "it must be remembered that PCP is primarily a working clinical theory. The assumption of individuality is, in this context, a useful clinical heuristic" (p. 22).
} 
valuable here. That is, the development of a theoretical position for elaborating the way in which a person makes sense of the world should equally be able to take account for our own meaning making activities. Thus it is argued that any theoretical position seeking to offer a reasonable consideration of the business of being a person, must embody key concepts if it is to be considered reflexive and therefore worthwhile pursuing further. The person envisaged must be replete with personal agency, and at the same time embedded in an inherently social world with other people who have the same degree of agency. Kelly (1955) is equally aware of the need to capture this socially embedded nature of the individual person as outlined in the following passage.

If a man's private domain, within which his behavior aligns itself within its own lawful system, is ignored, it becomes necessary to explain him as an inert object wafted about in a public domain by external forces, or as a solitary datum sitting on its own continuum. If a man's existence in the public domain is ignored, our painstakingly acquired knowledge of one man will not help us understand his younger brother, and our daily psychological efforts will yield no increment to the cultural heritage (p. 39).

Although Kelly's (1955) position will later be challenged with regard to the reflexive nature of his theoretical approach, in principle it embodies many of the central theoretical concepts that are considered to be fundamental to the conceptual framework being developed by this thesis. In light of this, a further consideration at the Psychology of Personal Constructs is warranted.

\section{The Psychology of Personal Constructs: A Considered Sketch}

Prior to a deeper and more critical consideration of Kelly's (1955) work in the following chapter, a preliminary sketch of the central challenge to his work helps shape an appreciation of the direction that will be taken. As previously noted, it has been suggested that Kelly (1955) dealt inadequately with the phenomena of language and overlooked what Chiari and Nuzzo (2004) describe as "the crucial importance of language in the construction of realities and selves" (p. 57). More specifically Kelly is adamant that the construct ${ }^{22}-a$ structure that he undeniably considers to be the fundamental structure of being - is situated beyond the reach of language. As we will see, this raises the question as to how it is that the

\footnotetext{
${ }^{22}$ Although this thesis has much to say about the construct in this and the subsequent chapters, a definition here provides a foundation for going on productively at this point. Kelly (1955) describes the construct in the following way: "Man looks at the world through transparent patterns or templates which he creates and then attempts to fit over the realities of which the world is composed...let us give the name constructs to these patterns that are tentatively tried on for size. They are ways of construing the world" (p. 9).
} 
'inner' - a person's constructs - can indeed come to language as an 'outlook'. In fact, it is about the implications of locating the structures of this 'inner' beyond the reach of language that this thesis has most to say with specific regard given to the adoption of the Psychology of Personal Constructs as a theoretical foundation for the conduct of qualitative research. That is, how is it that a person can bring the construct to language for him or her self let alone another person if indeed the construct is beyond the reach of language as Kelly (1955) suggests. Thus, in developing a new approach that informs qualitative research, and in so doing elaborates Kelly's (1955) position, it will be in the direction of taking greater account of language that this thesis turns. In order to move toward this elaboration we first need to appreciate Kelly's (1955) The Psychology of Personal Constructs in greater depth.

The Psychology of Personal Constructs represents a protracted theoretical effort to catch a glimpse of the individual person going about the business of being human. Kelly (1955) contends that the conceptualisation of the individual in terms of "man-the-scientist" (p. 4) most effectively embodies the business with which individuals concern themselves. As a foundation to the notion of his Constructive Alternativism, Kelly asks the reader to afford each individual 'scientist' like characteristics. ${ }^{23}$ In acknowledging these characteristics he suggests we go on 'as if' each person develops theories, creates and tests hypotheses, in the pursuit of experimental evidence in order to develop his or her own theoretical position. Thus, rather than simply responding to stimuli, each person - with the aspirations of the 'everyday scientist' - seeks to predict and control, as well as confirm and disconfirm, aspects of his or her developing understanding of the world. Therefore, for Kelly (1955) any theory or interpretation is only ever interim given the possibilities that new evidence provide. In light of this evidence, be it confirming or disconfirming, each theory can be adjusted accordingly. An adjusted theory therefore provides a different interpretation or a new personal theoretical position. ${ }^{24}$ For Kelly (1955) the application of the 'scientist' metaphor culminates in a question. Beginning from the notion that each person develops or erects for him or herself a theory to predict and control, to the best of his or her ability, the world around them, "might not the differences between the personal viewpoints of different men

\footnotetext{
${ }^{23}$ Kelly (1955) had much to say about what he considered to be the shortcomings of the dominant behaviourist model of the person at the time. He considered these approaches to be lacking in their ability to apprehend the person as being anything more than merely a biological organism that responds to stimuli or to their environment. In contrast, Kelly's 'scientist' metaphor suggests that individuals develop for themselves theories through which they are able to understand and represent the environment as opposed to simply responding to it.

${ }^{24}$ One could be mistaken for believing Kelly's (1955) position is simply subscribing to a form of idealism. However he is quick to establish that it is indeed "a real world" (p. 6) that he is talking about, not a world merely made up of people's thoughts.
} 
correspond to the differences between the theoretical points of view of different scientists?" (p. 5$)^{25}$

\section{The Construct}

These theoretical points of view are thus what Kelly describes as constructs. The following passage from Kelly (1955) provides a useful introductory description of the construct and a foundation for further consideration.

Man looks at his world through transparent patterns or templates which he creates and then attempts to fit over the realities of which the world is composed. The fit is not always very good. Yet without such patterns the world appears to be such an undifferentiated homogeneity that man is unable to make any sense out of it. Even a poor fit is more helpful to him than nothing at all. Let us give the name constructs to these patterns that are tentatively tried on for size. (pp. 8-9)

This description underpins the ontological significance of the construct for Kelly, as the most primary structure of going about the business of being human. In addition, it emphasises that the construct can be adjusted to provide a better 'fit' with reality if it is deemed necessary. Kelly (1955) writes that, "the world keeps rolling along and revealing these predictions to be either correct or misleading" (p. 14), determining ultimately what he describes as a constructs "predictive efficiency" (p. 12). The construct therefore provides a representation of the world, that is then tested upon the reality of the world in order to determine the degree to which the construct was actually capable of predicting that reality. It is important to appreciate therefore that a person's constructs represent a transient take on the world. It is a take on the world that one makes adjustments to, or may even abandon completely, in the light of new and contradictory evidence.

Challenging the conventions of traditional logic that considers concept formation as the degree to which certain things are naturally alike, Kelly assumes that there is much to be gained through a consideration also of that which is different. Kelly (1955) believes that a construct that implies similarity without any recourse to those elements of contrast would render "as much of a chaotic undifferentiated homogeneity as a construct which implied contrast without similarity would represent a chaotic particularized heterogeneity" (p. 51).

\footnotetext{
${ }^{25}$ It will be shown in the subsequent chapter that the abstraction of difference between the theoretical points of view of different people poses an untenable conundrum given that the construct - as the fundamental structure of one's theoretical position - is suggested to have its being beyond the reach of language.
} 
He also suggests that a consideration of that which is different corresponds more closely with observations of how a person actually thinks. Thus, the construct in its basic form is a consideration of the way in which at least two things are considered or construed as being similar while being construed as different from a third. Importantly, the element with which the two things are considered similar should be the same element with which the third is different. For example, Kelly (1955) states, "If we are to express a true construct: Mary and Alice are gentle; Jane is not" (p. 111), thus in so doing emphasises the way that a construct is formed as a bipolar structure - gentle $v s$. not gentle - on the basis of one common element gentle.

The example of a construct that is referred to frequently by Kelly, is the common dichotomy 'black $v s$. white'. Through the lens of a traditional logic that has similarity as its focus of consideration, something considered other than 'black' could only be 'not black'. However, for Kelly, the opposite of 'black' for a particular person may be 'white', establishing the construct 'black vs. white'. This construct may allow that person to bring very specific aspects of the world to understanding, for example, the colour of the sky, or even his or her mood. For another person, however, the opposite of 'black' may be 'AngloSaxon', highlighting a vastly different aspect of the world that the notion of black brings to understanding for that particular person. These two examples highlight the power of gaining an appreciation of that which is different when seeking to understand those aspects of a person's mental processes that he or she uses to bring an aspect of the world to understanding. Following from this line of thinking, in the process of forming a construct - i.e. construing it is not the entity being construed that has created the structures of the construct rather it is the individual construer (Kelly, 1955).

Continuing with the 'black $v s$. white' construct; beyond providing considerations of what it is that the weather is doing or perhaps a person's mood, this construct may seem to have limited applicability to other aspects of a person's life. Kelly (1955) termed the point at which a construct was deemed to be of limited use a construct's range of convenience. This describes the point at which a construct no longer provides a useful means of discriminating similarity and difference amongst aspects of the world that are to be understood. The aspects of the world that are located within a construct or those aspects for which the construct has applicability are termed a construct's context. For example, to continue again with the 'black $v s$. white' construct as a vehicle to discriminate mood, it is 'a person's mood' that represents the context for the construct, while that person who uses a 'black vs. white' construct for discriminating the weather situates 'the weather' as the context for that construct. Here it is clear that it is the individual person who creates and determines both the construct itself and 
the context within which it finds meaning and applicability. Kelly (1955) emphasises this in the following passage.

It is important for us to keep in mind that it is not the accumulation of the elements in the context that constitutes the construct, nor is it the differential grouping of the elements. Rather, the construct is the basis upon which elements are understood. It is a matter of how the person construes the elements in order to deal with them, not where they happen to appear or where he decides to set them down. The construct is an interpretation of a situation and not itself the situation which it interprets. (pp. 109-110)

Elaborated in such a way, the very nature of the construct gives the impression of offering little more than idealised conceptions with no connection to a reality beyond a person's constructions of it. However Kelly (1955) moves to situate each construct as merely one component of a greater, integrally related system of constructs, and in so doing he seeks to close down the idea that constructs can simply "be tossed about willy-nilly" (p. 15) despite being determined in every way by the individual person.

Not only do individual people differ by way of the constructs they develop, they also differ in the way in which they organise their constructs. It is for Kelly (1955) the way in which a person organises his or her constructs that genuinely represents that person as opposed to any individual construct itself. Further, the organisation of constructs into a greater, integrally related system of constructs means that a change in one construct produces a change in other constructs within that system. Thus, each construct exhibits an ordinal relationship with those other constructs that are operating 'in-series' with the construct in question. That is, each construct is at the same time subordinate to the construct(s) that determines it, while superordinate to and therefore determinate of other construct(s). It is important to recall that it is the person to whom Kelly (1955) has deferred agency in the establishment of his or her own system of constructs. Our greater construction system is therefore not a fixed entity that is resistant to change, but is rather, as Kelly (1955) identifies here, designed for a person's convenience.

One person may subsume the constructs A and B under the construct of C. Another may subsume $\mathrm{B}$ and $\mathrm{C}$ under $\mathrm{A}$. In fact, this kind of upsetting of the hierarchical apple cart characterizes much of our day-to-day thinking...one builds a system embracing ordinal relationships between constructs for his personal convenience in anticipating events. (pp. 52, 58) 
When Kelly (1955) made it clear that one could not merely 'toss' his or her constructs around "willy-nilly" (p. 15), he was alluding to what he considers to be an inherent stability within a person's construct system. Here Kelly suggests that it is the more superordinate elements in a person's system of constructs that ultimately determine those constructs that they subsume and therefore provide a degree of consistency to the broader system. Kelly (1955) uses the terms "core construct" and "peripheral construct" (p. 482) to elaborate this notion of construct system stability.

For Kelly (1955) “Core constructs are those which govern a person's maintenance processes - that is, those by which he maintains his identity and existence" (p. 42 emphasis in original). Core constructs, then, are considered to be comprehensive with regard to the functions they serve the person. They are largely resistant to change, thereby providing a significant degree of consistency and organisation to a person's construct system. ${ }^{26}$ Contrasting core constructs with peripheral constructs, Kelly (1955) describes these latter constructs as "those which can be altered without serious modification of core structures" (pp. 482-483, emphasis in original). It follows therefore, that adjustment to a peripheral construct is less complicated and has far fewer consequences than the adjustment of a core construct. ${ }^{27}$ All of this means that a person's constructions are open to varying degrees of revision and change. ${ }^{28}$ Despite this, as will be considered in detail shortly, the supposedly languageless nature of the construct makes this movement of revision and subsequent change theoretically challenging. A consideration of the movement of construct revision as elaborated by Kelly (1955) is important for highlighting some of the theoretical challenges that will need to be overcome by the theoretical position envisioned by this thesis.

\footnotetext{
${ }^{26}$ From a clinical psychology perspective it is this consistency between a person's constructs that relates most closely to the degree of consistency within that person's personality.

${ }^{27}$ Kelly (1955) goes on to elaborate the notions of 'tight' and 'loose' construing as a consideration of the degree to which a person is able to or likely to make change to his or her construct system. In addition, Kelly (1955) elaborates the importance of core constructs in considering common psychological phenomena such as 'threat' (pp. 489-494), 'fear' (pp. 494-495), 'anxiety' (pp. 495-502), 'guilt' (pp. 502-508), 'aggressiveness' (pp. 508-510) and 'hostility' (pp. 510-514). This discussion is not taken up by this thesis given that the focus of consideration here is upon the capacities of qualitative research as opposed to those of psychotherapeutic practice.

${ }^{28}$ Kelly follows on from the ideas of 'core' and 'peripheral' constructs through a consideration of the notions of 'control and determinism'. For Kelly these notions are not absolute states, but rather the opposing poles of the same construct. Thus, they are dependant upon the way in which an individual person construes an event. As Kelly (1955) suggests, "that which is subsumed by a construct may be seen as determined by it; that which subsumes the construct is free with respect to it" (p. 126).
} 


\section{Construct Revision}

As has been mentioned previously, the process of building a repertory of constructs requires that each person test his or her constructs against reality and make adjustments to them in the hope of achieving a better fit. As Kelly (1955) put it,

In general man seeks to improve his constructs by increasing his repertory, by altering them to provide better fits, and by subsuming them with superordinate constructs and systems. In seeking improvement he is repeatedly halted by the damage to the system that apparently will result from the alteration of a subordinate construct. (p. 9)

Kelly (1955), identifying the construct as "a representation of the universe, a representation erected by a living creature then tested against the reality of the universe" (p. 12), locates the construct as an anticipatory structure, that therefore anticipates the reality that is to come. The success or otherwise of this anticipation is dependent upon an assessment of 'fit' with reality. The following passage from Kelly (1955) captures how it is that a person tests his or her constructs for fit.

When a person scans the events with which he is surrounded he "lights up" certain dichotomies in his construct system. Thus construct systems can be considered as a kind of scanning pattern which a person continually projects upon his world. As he sweeps back and forth across his perceptual field he picks up blips of meaning. The more adequate his scanning pattern, the more meaningful his world becomes. (p. 145)

Kelly (1955) recognises that we would be "hopelessly bogged down" (p. 13) without the opportunity to adjust our original forecasts. That is, to make changes to our constructs in light of the 'blips' or the "validational evidence" (p. 451) that Kelly believes should be continually sought for our constructs; a practice that "characterizes any alert person" (p. 13). Problematically, however, if constructs are indeed beyond the reach of language, (as will be explored in the next chapter), it is theoretically inconceivable as to how or by what means we would engage in the adjustment of a construct itself. A considered exploration of the elemental workings of The Psychology of Personal Constructs will provide a foundation for considering the theoretical implications of such problems as well as pointing toward those aspects of Kelly's (1955) theoretical position that call for further elaboration. Here, then, it is worth delineating the Fundamental Postulate and its accompanying eleven corollaries in detail, making further reference to the potential problem of Kelly's (1955) inadequate account of language. 


\section{The Fundamental Postulate}

A PERSON'S PROCESSES ARE PSYCHOLOGICALLY CHANNELIZED BY THE WAYS IN WHICH HE ANTICIPATES EVENTS (Kelly, 1955, p. 46). ${ }^{29}$

The Fundamental Postulate represents for Kelly a tentative or interim statement through which the carriage of the theoretical tenets of his constructive alternativist approach can be achieved. As already mentioned, Kelly (1955) emphasises that his first consideration is the individual person, over and above any groups of people. This person is credited as being an active being and not merely "an object which is temporarily in a moving state" (p.48). Kelly is clear that his concern is with the psychological consideration ${ }^{30}$ of the person's processes and that these processes are 'channelized' by a network of pathways provided by that person's construct(s) and his or her greater construct system. Thus, a person's constructs provide the 'grooves' through which these processes operate, grooves that are established prior to an event in the oncoming world, and are therefore anticipatory in nature. $^{31}$ Within the elaboration of his Fundamental Postulate, Kelly (1955) thus carries out an important manoeuvre in an attempt to ground the psychological functioning of the individual person within reality. In so doing, he attempts to overcome any subsequent charge of relativism. Kelly (1955) suggests that, "man ultimately seeks to anticipate real events" (p. 49). In other words, the anticipations or constructs that a person develops - through a movement of abstraction - are laid upon the world and assessed with regard to their predictive efficiency or their degree of fit with the reality of the world itself. Although Kelly here has clearly established the psychologically proactive nature of the person that he conceives of, the following chapter will show that locating the construct beyond the reach of language makes this fundamental postulate a theoretically difficult position to support. That is, in the absence of the medium of language - required in order for each of us to undertake the movement of abstracting - it is counter intuitive to apprehend that we could recognise the degree to which our constructs do indeed anticipate the actuality of the world.

\footnotetext{
${ }^{29}$ In Kelly's (1955) original work the Fundamental Postulate and elaborative corollaries are all capitalised. In keeping with this tradition this thesis continues the use of this capitalisation.

${ }^{30}$ Kelly (1955) takes the view that no single discipline can own the study of a human process. Instead he suggests that what disciplines should recognise is that they simply investigate these processes from a particular perspective. For example, Kelly (1955) believes it worthwhile to consider a person's processes psychologically, rather than consider that the processes themselves as necessarily being psychological in nature.

${ }^{31}$ Kelly (1955) states that "anticipation is the push and pull of the psychology of personal constructs" (p. 49). In so doing, he encompasses the notion of motivation - despite being openly opposed to motivation - by suggesting that what each person seeks to produce are constructs that have a better anticipatory fit with the world.
} 


\section{Construction Corollary}

A PERSON ANTICIPATES EVENTS BY CONSTRUING THEIR REPLICATION (Kelly, 1955, p. 50).

Building upon the psychologically proactive nature of the person, this corollary provides Kelly (1955) a means of further emphasising that it is the individual person who produces a network of constructs through which the substance of the world "takes shape or assumes meaning" (p. 50). Importantly, it is not the substance that creates the network of constructs but the individual person. Recall that this application of a construct to a substance or event that is to be understood, is the process that Kelly describes as 'construing'. Here a person notes elements of similarity and elements of difference, and it is this similarity and difference that is the hallmark of a construct's bipolarity. For Kelly (1955) the notion of replication provides the basis upon which we distinguish between aspects of similarity and aspects of difference in the development of our constructions. He is clear that the use of the term replication does not support a view whereby an event is merely a duplicate of a previous event. Rather, replication here is a consideration of those aspects of an event that have been identified with sufficient consistency in order to satisfactorily predict aspects of events that are yet to come. ${ }^{32}$ The following passage from Kelly (1955) identifies that the effects of time alone make the duplication of events impossible.

An event is replicative of another only if one is willing to accept the abstracted similarity of the two. Thus a person who owns one cow and one horse may say that he owns two animals - if he is willing to accept the animal-like abstraction of the two of them. (p. 53)

It follows, then, that events can be considered replicative to the extent that we can distinguish a level of abstraction with which to consider elements of these events as alike as well as elements that are different. Hence, when a person anticipates events by construing their replication, what is being construed is the extent to which an event can be abstracted at a level that is consistent with what is already within that person's construct repertoire. Again the requirement for a person to make abstractions between a construct - as the structure through which the substance comes to meaning - and the substance itself, certainly suggests that the construct itself is able to engage, at some level, in the movement of abstraction. However, if we go on as if the construct has no recourse to language, as Kelly suggested, then, his idea certainly complicates the possibility of this abstraction.

\footnotetext{
${ }^{32}$ Kelly uses the notion of 'a day' to establish what he means by replication. Through the concept of time, Kelly indicates that tomorrow cannot be a duplicate of today, unless time could 'double back' on itself. Instead, the elements of a day - the sun rising, the sun setting - are in themselves unique events each day, despite being understood as a recurrent or replicated theme across days.
} 


\section{Individuality Corollary}

PERSONS DIFFER FROM EACH OTHER IN THEIR CONSTRUCTION OF EVENTS (Kelly, 1955, p. $55)$.

Kelly (1955) has been clear within the previous corollaries that it is with the individual person that he is most concerned. However, the notion is perhaps taken to its maximum in his indication that the groundwork of these corollaries provides a psychology of individual differences.

People can be seen as differing from each other, not only because there may have been differences in the events which they have sought to anticipate, but also because there are different approaches to the anticipation of the same events (p. 55).

$\mathrm{He}$ is clear that the foundation of 'individual difference' is a function of the Construction Corollary, where each person erects a series of constructs through which the world assumes meaning. Despite this, Kelly (1955) is quick to indicate that this inherent 'individual difference' does not foreclose on the possibility of people sharing experience, instead, "while there are individual differences in the construction of events, persons can find common ground through construing the experiences of their neighbours along with their own" (p. 56). For Kelly this common ground is a consideration of the extent to which one person construes the constructions - the construal of likeness and difference between events - of another person as being comparable with his or her own constructions. Importantly, the construal of another's constructions as similar, does not here suggest a sharing of the same constructs or necessarily the sharing of events themselves. ${ }^{33}$ It is the position of this thesis that if a person is to be considered different on the basis of anticipations - his or her constructs - then it follows that the person needs to be capable of bringing the construct(s) to light for consideration. In other words, we need to be able to bring of our construct(s) to language in order that we can make determinations about how our own constructs differ from those of other people. However, such determination of difference is a difficult prospect if the construct itself is indeed incapable of being brought to language.

\footnotetext{
${ }^{33}$ Kelly (1966/1970) later highlights his "doubt that two persons ever put their construction systems together in the same logical relationships" (p. 9).
} 


\section{Organization Corollary}

EACH PERSON CHARACTERISTICALLY EVOLVES, FOR HIS CONVENIENCE IN ANTICIPATING EVENTS, A CONSTRUCTION SYSTEM EMBRACING ORDINAL RELATIONSHIPS BETWEEN CONSTRUCTS (Kelly, 1955, p. 56).

Having established that each person is different with regard to his or her construction of events, Kelly goes further and suggests that the way these constructions are organised into a greater system of constructs is also determined by the individual person. Kelly (1955) suggests that "not only are the constructs personal, but the hierarchical system into which they are arranged is personal too" (p. 56). Recall how Kelly established that each person reaches forward in an effort to produce constructs that have a better 'fit' with reality. This corollary supports the idea that it is in fact the systematic adjustment of the position of a construct within a person's system of constructs that - to a greater extent - minimises inconsistencies and contradictions, and therefore supports a 'better fit' between the construct and reality. These constructive modulations within a greater system of superordinate and subordinate relationships - embodied by the Organisation Corollary - have widespread and significant implications for the clinical application of the fundamental tenets of the Psychology of Personal Constructs. ${ }^{34}$ Despite their importance, however, the abstraction that is required in order to systematically situate one construct in relation to another construct is palpably incommensurate within a structure that is itself beyond the reach of language.

\section{Dichotomy Corollary}

\section{A PERSON'S CONSTRUCTION SYSTEM IS COMPOSED OF A FINITE NUMBER OF DICHOTOMOUS} CONSTRUCTS (Kelly, 1955, p. 59).

The Dichotomy Corollary captures more completely the idea that inherent within a construct is a consideration of both similarity and difference. As mentioned previously, a person abstracts the element by which two events can be considered a replication and with which another event most certainly is not. In Kelly's (1955) words,

\footnotetext{
${ }^{34}$ As previously noted, the scope of this thesis is not sufficient to justify a consideration of the clinical implications of this position beyond a cursory note. For Kelly (1955) the suggestion that a person's constructs exist within a greater system of constructs provides the foundation for the task of a psychotherapeutic intervention that employs a Psychology of Personal Construct approach. If indeed it is through the system of constructs that a clinician and a client seek to minimise inconsistencies and contradictions, then an adjustment in that system should be commensurate with an adjustment of the fit between the client's constructs and reality itself.
} 
If we choose an aspect in which $\mathrm{A}$ and $\mathrm{B}$ are similar, but in contrast to $\mathrm{C}$, it is important to note that it is the same aspect of all three $\mathrm{A}, \mathrm{B}$, and $\mathrm{C}$, that forms the basis of the construct. It is not that there is one aspect of A and B that makes them similar to each other and another aspect that makes them contrasting to $\mathrm{C}$. What we mean is that there is an aspect, of $\mathrm{A}, \mathrm{B}$, and $\mathrm{C}$ which we may call $z$. (pp. 59-60)

Kelly (1955) suggests that all constructs adopt this basic dichotomous and 'bi-polar' form. Therefore, within the range of convenience of the construct are those elements that can assume meaning on the basis of similarity and difference. Here too those elements outside the range of convenience of the construct are "unrecognizable" by way of that construct (pp. 60-61). In other words, an aspect of the world can assume meaning by virtue of being recognised as similar and yet different in the same way as the elements of a specific construct. However, in order to undertake the abstraction of similarity and difference Kelly alludes to, one must surely bring to understanding that bi-polar criteria with which this similarity and difference is to be considered. That is, a person must bring his or her constructs to understanding in order to engage in abstraction, or a dialogue, with those aspects of the world that may or may not assume meaning through it. This dialogue of abstraction is theoretically challenging to establish and sustain within a system of languageless and yet fundamentally ontological structures.

\section{Choice Corollary}

\section{A PERSON CHOOSES FOR HIMSELF THAT ALTERNATIVE IN A DICHOTOMIZED CONSTRUCT}

THROUGH WHICH HE ANTICIPATES THE GREATER POSSIBILITY FOR EXTENSION AND DEFINITION OF HIS SYSTEM (Kelly, 1955, p. 64).

Stemming from the proposition that a person will always actively seek to better the fit of his or her anticipations or construct system with reality, the Choice Corollary posits that each person chooses one pole of a bi-polar dichotomous construct with which to anticipate the reality to come. ${ }^{35}$ The following passage from Kelly (1955) is explanatory.

We assume, therefore, that whenever a person is confronted with the opportunity for making a choice he will tend to make that choice in favour of the alternative which seems to provide the best basis for anticipating the ensuing events (p. 64).

\footnotetext{
${ }^{35}$ Kelly (1955) uses the term Elaborative Choice to apprehend the choice that the person has with regard to that end of the bi-polar construct he or she uses to anticipate reality (see pp: 65-67, 128, 410, 508).
} 
Thus for Kelly the choice that a person makes, concerning which end of the bi-polar construct with which to anticipate the oncoming reality, ultimately has as its goal the elaborative development of his or her construct(s) and greater construct system. That is, the development of the degree of 'fit' of that person's constructs with the future. Although the notion of elaboration may give the impression of a person testing new and perhaps extreme constructs, Kelly is clear that when a person elaborates his or her construct system, it is either in the direction of extension or definition.

Extension of a construct system makes that system more comprehensive by increasing the range of convenience of the construct and subsequently increasing those aspects of reality that are able to assume meaning within it. Kelly (1955) describes definition on the other hand as a process that seeks "constricted certainty" (p. 65) through the confirmation of previously tested aspects of a person's construction system. Irrespective of the direction with which a person envisages the greatest possibilities of elaborating his or her construct system, Kelly (1955) recognises the importance of this movement itself.

To our way of thinking there is a continuing movement toward the anticipation of events... and this movement is the essence of human life. (p.

It is perhaps this movement toward anticipation that represents for Kelly the fundamental human comportment. It represents the movement that compels the person going about the business of being human. However, it is the thinking of this thesis that if the construct is indeed a languageless structure, the way that a 'person chooses one pole' of a dichotomous construct in order to anticipate the oncoming reality is untenable, not to mention out of step with Kelly's theoretical position. In other words Kelly (1955) is clear that a construct is a dichotomous or bi-polar structure and that the construct has its being beyond the reach of language. However it surely follows that if a person is to make a determination of which pole of the 'black $v s$. white' construct that he or she will seek an elaboration of, then both poles of the construct, and therefore the construct itself, need to come to understanding in order that the appropriate abstractions between the two poles can be made. Therefore, despite Kelly's claim otherwise, the poles of a construct must be able to come to language in order that Kelly's (1955) position can be considered theoretically tenable. 


\section{Range Corollary}

\section{A CONSTRUCT IS CONVENIENT FOR THE ANTICIPATION OF A FINITE RANGE OF EVENTS ONLY}

(Kelly, 1955, p. 68).

As mentioned previously, each construct has its own range of convenience or a set of boundaries beyond which elements of the world cannot assume meaning. A passage from Kelly (1955) is here explanatory.

A construct of tall vs. short is much easier to see as having a limited range of convenience. One may construe tall houses versus short houses, tall people versus short people, tall trees versus short trees. But one does not find it convenient to construe tall weather versus short weather, tall light versus short light, or tall fear versus short fear. Weather, light, and fear are, for most of us at least, clearly outside the range of convenience of tall vs. short. (p. 69)

It must be recalled that both similarity and difference are inherent within the same construct. Thus, for Kelly (1955), something outside the range of convenience does not simply represent that which is in contrast to the elements that $d o$ assume meaning, instead it is "an area of irrelevancy" (p. 69). Kelly's elaboration of the Range Corollary is explicit about the equal importance of gaining an appreciation of the contrasting end as well as the similarity end of the bi-polar structure. Underpinning this position is a belief that a person cannot begin to understand completely the meaning that an element of the world assumes unless he or she appreciates what is envisaged as relevantly opposed. Considering a person's use of the construct 'respect $v s$. contempt', Kelly (1955) elaborates this notion.

When we approach [a person's construct] thinking from the standpoint of the psychology of personal constructs, we do not lump together what he excluded as irrelevant with what he excludes as contrasting. We see the construct as composed essentially of a similarity-contrast dimension that he strikes through a part of his field of experience. We need to look at both ends if we want to know what it means to him. We cannot understand him well if we look only at the similarity - 'respect' - end of the dimension. We cannot understand what he means by 'respect' unless we know what he sees as relevantly opposed to 'respect.'....He would therefore seek to understand what his client construed as the opposite of respect and what the range of convenience of the whole construct covered. (p. 71) 
The Range Corollary embodies Kelly's challenge to theoretical approaches that deem as irrelevant anything that cannot be conceptualised by way of similarity. From the perspective of this thesis the fundamental structure of the construct, encompassing both similarity and difference, provides fertile ground for achieving a greater depth of understanding in qualitative research. Despite this, if the construct is not able to come to language, as Kelly (1955) suggests, this makes the illumination of the respective poles of the construct - and therefore the construct itself - a difficult theoretical position to maintain.

\section{Experience Corollary}

\section{A PERSON'S CONSTRUCTION SYSTEM VARIES AS HE SUCCESSIVELY CONSTRUES THE} REPLICATION OF EVENTS (Kelly, 1955, p. 72).

The Fundamental Postulate establishes that the anticipation of events is the cornerstone of a person's psychological processes. The Experience Corollary is an extension of this position and identifies experience as the process of evolving these anticipations. Commensurate with the psychologically proactive person that Kelly (1955) envisages, he suggests that the events of the world "continually subjects a person's construction system to a validation process" (p. 72). The process of validation itself may reveal a less suitable 'fit' between a person's anticipations and reality. The recognition of this poor fit therefore requires a progressive and developmental evolution of that person's construction(s). It is the movement from an initial construction - found to be of poor fit - to a new construction, through a process of 'reconstrual', that epitomises what experience is for Kelly. He is clear that experience itself involves active mental processes. In fact, genuine experience is epitomised by the process of making something of the events of the world, beyond merely the passage of the events themselves. The following passage is exemplary of Kelly's (1955) conception of experience.

A person can be a witness to a tremendous parade of episodes and yet, if he fails to keep making something out of them, or if he waits until they have all occurred before he attempts to reconstrue them, he gains little in the way of experience from having been around when they happened. It is not what happens around him that makes a man experienced; it is the successive construing and reconstruing of what happens, as it happens, that enriches the experience of his life. (p. 73)

Thus experience is the culmination of that which is made of the events and a person's progressive revisions of his or her constructions. Or, in Kelly's (1955) words, experience, "is a set of personally construed events...the extent of what we know - up to now" (p. 171). We 
must recall that in the movement of construing, a person abstracts an element - albeit without language - which can be used to determine the degree with which these events can be considered to be replicated. ${ }^{36}$ Kelly goes on to highlight that when a person identifies a recurrent theme, and therefore a degree of orderliness or consistency between events, he or she begins to experience them. He goes on to suggest that experience plays a validating role with regard to a person's construct(s) and construct system. That is, Kelly (1955) recognises that a person knows a lot of things that are untrue, and in continuing to take his or her constructions for granted and not seeking new "light to throw upon them, adds very little to his store of experience as the years go on" (p. 172). Thus, the anticipatory, as opposed to reactive, nature of experience with which Kelly (1955) is concerned is couched comfortably in the following terms.

That portion of the universe which is happening to us - that is, which is successively construed by us - and the increase of experience is a function, not of the hodgepodge of events which we have construed, or of the time spent in being aware of them, but of the successive revision of our construct system in the general direction of increased validity. (p. 172)

Importantly, the process of constructive revision that underpins experience always involves a person being 'pulled up short' or dissatisfied in some way with the 'fit' of his or her constructs with the anticipated reality. If we take Kelly's suggestion that the construct bears no connection to language, then his notion of genuine experience is difficult to sustain. In order to have an experience a person must make something of his or her constructs. In other words, that person must reconstrue. However, without a medium such as language within and through which to engage in a movement between reality and one's constructs, makes it difficult to apprehend the way in which a person actually does make something of the events of the world.

\footnotetext{
${ }^{36}$ It is important to keep in mind that the term replication does not represent for Kelly a complete repetition of the same event, rather is a replication of an anticipated element. For example, and as outlined in a previous footnote, each day the sun rises and sets. Although it is indeed a different day, some overlapping elements are in fact replicated. Kelly (1955) suggests that as a function of time alone, an event cannot be replicated.
} 


\section{Modulation Corollary}

THE VARIATION IN A PERSON'S CONSTRUCTION SYSTEM IS LIMITED BY THE PERMEABILITY OF THE CONSTRUCTS WITHIN WHOSE RANGES OF CONVENIENCE THE VARIABLES LIE (Kelly, 1955, p. 77).

It would be theoretically inconsistent for Kelly (1955) to first acknowledge that those elements within the world that are able to lawfully assume meaning for a person do so only in terms of "what his framework is designed to permit him to see in the stimuli" (p. 79), and then fail to capture the way in which change within this system can lawfully evolve. For this reason the Modulation Corollary encompasses the way that changes to an individual construct - through the process of experience - can be embodied within a greater construction system. The degree to which a person's construct system is able to lawfully embody new elements is conceived of in terms of a construct's permeability. The following passage provides further detail to Kelly's (1955) notion of permeability.

A construct is permeable if it will admit to its range of convenience new elements which are not yet construed within its framework...a construct can be called permeable if it is so constituted that new experience and new events can be discriminatively added to those which it already embraces. A construct which "takes life in its stride" is a permeable one. (pp. 79, 81)

Recall, by way of the Organisation Corollary, that Kelly proposed that a person's constructs exist within a greater system of constructs that are integrally related to each other. It is important to appreciate from that discussion that the more core a construct is, the more it subsumes other constructs. However, the function of a construct's ability to subsume other constructs is not to be confused with a construct's degree of permeability. In fact, it is more likely that the more core a construct is within a person's system of constructs the less permeable it is. On the other hand, a construct that is permeable is, according to Kelly (1955), a construct that is open to a variety of "experimental ventures" (p. 81) and is therefore able to embrace those things that the person has not as yet thought of. However the Modulation Corollary, although representing the flexibility of the construct system, also embodies the degree of stability that exists within a person's construct system itself. That is, a change in a construct and the introduction of a new construct, can only occur if an existing construct is sufficiently permeable to subsume it. It is in fact this requirement that maintains a degree of consistency or stability in a person's greater construct system.

The position proposed by this thesis suggests that the evolution of an individual construct and the expansion of a person's greater construct system involves an engagement in 
a dialogue between those events that are to be understood and his or her constructs. Thus, in order for a person to determine the range of convenience of a construct, as well as the degree to which a construct is permeable, requires that one engage with the construct itself.

However, Kelly's neglect to disclose a medium through which this dialogue could occur, and more directly, to adamantly situate the construct beyond the reach of language makes the possibility of this dialogical engagement unsustainable.

\section{Fragmentation Corollary}

\section{A PERSON MAY SUCCESSIVELY EMPLOY A VARIETY OF CONSTRUCTION SUBSYSTEMS WHICH} ARE INFERENTIALLY INCOMPATIBLE WITH EACH OTHER (Kelly, 1955, p. 83).

The elaboration of the Modulation Corollary ${ }^{37}$ supports an assumption that a person's construct system exhibits a degree of stability or consistency. Kelly emphasises, however, that this stability should not be confused for a logically intact and internally consistent system. On the basis that human life itself is poorly represented by an appeal to such a locktight consistency, for Kelly (1955) the Fragmentation Corollary offers "a satisfactory answer to the important psychological question of how the human organism can be organized and still appear to behave in a disorganized fashion" (p. 88). Kelly (1955) recognises the possibility that one could argue that an internal inconsistency exists between the Modulation and Fragmentation Corollaries. One corollary assumes a degree of consistency while the latter assumes a degree of inconsistency. However Kelly (1955) goes on to describes the inherent synergism that exists between these corollaries.

The Fragmentation Corollary follows as an explicit statement of the kind of inconsistency which the Modulation Corollary implicitly tolerates. The Modulation Corollary tolerates inconsistency between subsystems. More specifically it tolerates the successive use of the subsystems which do not, in themselves, add up. (pp. 87-88)

Here Kelly is suggesting that each person can come to conclusions about the same series of events that are inherently inconsistent with each other. These differences in conclusions are a product of the different levels of construct - more subordinate or more superordinate employed to make meaning of the event. However, the degree of this apparent disorganisation in a person's conclusions is ultimately determined by the more influential elements of his or her system of constructs. Thus, it is ultimately these more superordinate as

\footnotetext{
${ }^{37}$ Kelly (1955) makes an important statement that is not seen within the unpacking of his other corollaries, that "this is an important corollary" (p. 83).
} 
well as the permeable features of a person's construct system that give their life overall meaning.

With regard to being theoretically reflexive, the Fragmentation Corollary is an effort to embody reflective contemplation. Many have experienced the effect of trying to contemplate a situation from a different perspective or a different point of view. In Kelly's terms, this contemplation is a process of envisaging a situation from the perspective of a different construct. Again the movement required of this contemplation most certainly involves the construct(s) - albeit inferentially incompatible - coming to thought in the medium of language; the very position from which this contemplation originates. However, without proposing a medium within which the construct has its being, it is difficult to contemplate the way in which Kelly envisages a person reaching a conclusion about the utility of his or her constructs, not to mention the subsequent place of these constructs within the greater system of constructs. In other words, if the construct is not consistent with language at some level, then, the way in which a person maintains his or her construct system's overall integrity and consistency is theoretically incoherent. It follows therefore, that, if the construct cannot come to be meaningfully understood outside of language, then a person cannot engage in the level of abstraction that is most certainly required here.

\section{Commonality Corollary}

TO THE EXTENT THAT ONE PERSON EMPLOYS A CONSTRUCTION OF EXPERIENCE WHICH IS SIMILAR TO THAT EMPLOYED BY ANOTHER, HIS PSYCHOLOGICAL PROCESSES ARE SIMILAR TO THOSE OF THE OTHER PERSON (Kelly, 1955, p. 90).

The Experience Corollary emphasised that Kelly sees experience as the resultant effort of the construing individual, rather than merely a person's exposure to events themselves. The Commonality Corollary takes this position further and embodies a commonality between people on the basis of their construction processes and not merely by way of their exposure to events themselves. Here Kelly (1955) uses the terms construction of experience as the determinant characteristic of similarity between the mental processes of individual people.

To construe experience, then is to take stock of the outcomes of this successive construing process. Thus, if two people take similar stock of their successive interpretations, their behavior will exhibit similar characteristics. The historical development of their thinking need not be similar - only the stock-taking need be similar. Hence it is not the similarity of experience 
which provides the basis for similarity of action, but similarity of their present construction of that experience. (p. 92)

Therefore, it is the degree to which one person construes his or her own experience as similar to the constructions of another person that underpins the Commonality Corollary. Here Kelly recognises that some of the real events that a person anticipates in going about the business of being human are the behaviours of other-persons. Thus, through the Commonality Corollary he begins to take the Psychology of Personal Constructs into the domain of interpersonal relations. For Kelly (1955), the foundation of this relationship is not a similarity of behaviour, nor a similarity on the basis of experiencing the same events, nor yet again, a similarity in the utterance of the same verbal labels "given that a person may construe his experience with little recourse to words" (p. 92). Instead, it is a similarity on the basis of construing experience in the same way.

The Commonality Corollary identifies a clear recognition that one person bring into the open his or her constructions in order to coalesce with the constructions of another person as a means of determining a degree of commonality. Again, it must be said, that if the construct bears no resemblance to language, the tenets of the Commonality Corollary would appear to be difficult to sustain. This view is underscored all the more by Kelly's (1955) own suggestion that "a person may construe his experience with little recourse to words" (p. 92).

\section{Sociality Corollary}

TO THE EXTENT THAT ONE PERSON CONSTRUES THE CONSTRUCTION PROCESSES OF ANOTHER, HE MAY PLAY A ROLE IN A SOCIAL PROCESS INVOLVING THE OTHER PERSON (Kelly, 1955, p. 95).

Continuing the conceptualisation of the person as being 'in relation' with other actively construing persons, the Sociality Corollary ${ }^{38}$ suggests that in order to have a social relationship people do not have to construe things as others do, but rather should gain an appreciation of the way in which another person sees things. In Kelly's (1955) words, "the person who is to play a constructive role in a social process with another person need not so much construe things as the other person does as he must effectively construe the other person's outlook" (p. 95). Therefore, as an elaboration of the Fundamental Postulate, the Sociality Corollary establishes the subsuming of another person's construing efforts as the

\footnotetext{
${ }^{38}$ Leitner and Thomas (2003) suggest that, "Kelly considered the Sociality Corollary his most important theoretical statement" (p. 257). We must however recognise that it was not Kelly's (1955) Fundamental Postulate.
} 
basis of social interaction. Kelly (1955) believes this position provides the beginnings of a social psychology founded upon the tenets of interpersonal understanding.

Here we have a take-off point for a social psychology. By attempting to place at the forefront of psychology the understanding of personal constructs, and by recognizing, as a corollary of our Fundamental Postulate, the subsuming of other people's construing efforts as the basis for social interaction, we have said that social psychology must be a psychology of interpersonal understanding, not merely a psychology of common understanding. (p. 95)

Fundamental to the achievement of this interpersonal position and the psychotherapeutic aspects of the Psychology of Personal Constructs, Kelly also emphasised a key consideration of role. Specifically Kelly (1955) suggested that in a social process each person adopts a role in relation to another person. This role enables each person to subsume the construing efforts of another person within his or her greater construction system.

A role is a psychological process based upon the role player's construction of aspects of the construction system of those with whom he attempts to join in a social enterprise... a course of activities which is played out in the light of one's understanding of the behavior of one or more other people. (pp.

97,100)

Here Kelly is clear that for him a social process is not a movement whereby one person seeks to adopt the constructions of another person in such a way that they become his or her own. Instead, it is the adoption of a role in relation to the way in which one person construes the construction processes of another person. Thus, for Kelly, a role enables each person to catch a glimpse of the way in which another person construes his or her reality; it is an opportunity to understand this perspective without having to necessarily share it.

At the core of the Sociality Corollary is a recognition that the individual person Kelly envisages is not isolated from other actively construing people. However, the fundament of human coalescence is the requirement of a shared medium within and through which this coalescence takes place - language. If the construct has its being beyond the reach of 
language, then the possibility of 'the subsuming of other people's construing efforts as the basis for social interaction' is theoretically problematic. ${ }^{39}$

\section{Theoretical Juncture}

It is at this juncture that the Fundamental Postulate proposed by the Psychology of Personal Constructs achieves its elaboration. It represents for Kelly (1963/1995) a "protracted effort to catch a sense of man going about the business of being human, and what on earth it means to be a person" (p. 183). For this thesis, in turn, it represents a theoretical position for the purpose of developing a different theoretical approach for qualitative research. An approach that is better able to catch a glimpse of the socially embedded person actively going about the business of being human. It is hoped that that this position will provide a deeper view of that person at the level of the 'inward outlook'. Nonetheless, despite the parallels of Kelly's work with the theoretical intentions of this thesis, one must recall the previous contention that the Psychology of Personal Constructs has inadequately dealt with the notion of language.

If this were so, Kelly's (1955) theoretical position can be challenged upon two fronts. Firstly, given that the construct has its being beyond the reach of language - as Kelly himself insists, indeed the focus of the following chapter - then his Fundamental Postulate and many of the amplifying corollaries seem theoretically problematic. Secondly, in situations where a person, for example a clinician, assumes to have brought another person's constructs to language ${ }^{40}$ for the purpose of clinical practice - that clinician can be challenged for having

\footnotetext{
${ }^{39}$ It is important to establish clearly that this thesis is not pursuing the 'well trodden' argument that suggests that Kelly (1955) does not account for the social aspects of being human. Although described by Warren (1998) as predominately intrapsychic in nature, for a consideration of the social dimensions of personal construct psychology see Kalekin-Fishman and Walker (1996) The Construction of Group Realities, as well as Chiari and Nuzzo (2003a) Psychological Constructivism and the Social World. In contrast to pursing the social versus the personal argument further, this thesis contends that Kelly has not adequately dealt with language and what's more he actively situates the construct beyond the reach of language. The implications for the Commonality and Sociality Corollaries are yet to be seen. However, it must be underscored that this suggestion is not a direct challenge aimed at Kelly's attendance or otherwise to the social dimension of being human, but rather is a challenge to the theoretical reflexivity of his position in the absence of language. This represents a significant difference and is one worthy of appreciation.

${ }^{40}$ At various points throughout his work Kelly (1955) mentions the following constructs 'good $v s$. bad', 'intelligent $v s$. stupid', 'light $v s$. dark', 'evaluative $v s$. descriptive' (p. 57). In so doing, he provides a few examples of where the construct can, and does indeed appear to come to language. Thus, these constructs must have miraculously come to the reach of language. In other words, if we adhere to the way that Kelly (1955) actively locates the construct beyond the reach of language, and therefore that the construct itself is something different from language and yet he is able to bring the construct to language as text, then it would appear that Kelly himself has slipped into adopting a naïve view of language of some form. Given that for Kelly (1955) then, the construct itself is not contingent upon language, or that language is not disclosing of the construct, the words that a person uses to describe a construct must simply point toward the construct. Once again, however, this is made all the
} 
slipped into assuming a naïve view of language and meaning, the same pervasive assumption that has been argued above to undermine representation within qualitative research. That is, in order for Kelly's (1955) approach to be theoretically sustainable and to produce supposedly legitimate representations of the people encountered in clinical practice and the like, one must accept that language is able to simply point to things - in this case a person's constructs. However, given that Kelly (1955) is adamant that the construct is beyond the reach of language, questions concerning the likelihood that it is indeed a construct that language is pointing towards seem legitimate.

\section{Open to Elaboration}

Unquestionably Kelly sought to provide a take on the psychological processes of a proactive individual going about the business of being human. However, the way in which he appears to have situated the construct - as the fundamental ontological structure with which he concerned himself - as having no relation to language, and therefore beyond the reach of language, opens up to question the theoretical tenability of many of the psychological processes that Kelly proposes. Despite this, many of the theoretical principles within Kelly's work have sufficient parallels with the intentions of this thesis to warrant further contemplation. ${ }^{41}$

One could argue that the focus upon qualitative research with which this thesis is concerned is outside the range of convenience with which Kelly concerned himself. However, the pursuit of an approach to qualitative research that elucidates a person's experience, at the level of a genuine 'inner outlook', highlights some clear parallels and intersections with the psychological functioning of the person that Kelly was most centrally concerned. ${ }^{42}$ It is these parallels that underscore the productive possibilities of an elaboration of Kelly's (1955) theoretical position in the direction of the aims of this thesis. In addition, the possibility of the theoretical developments proposed here sits comfortably with Kelly's (1955) own invitation for theoretical development. That is, Kelly (1955) suggests that the

\footnotetext{
more theoretically unsustainable in light of the way that Kelly (1955) separated the construct from language all together.

${ }^{41}$ There are several principles of Kelly's work that this thesis considers to be most productive. Firstly, that the individual is the proactive constitutor of the world. Secondly, the deep level with which the structures of this proactivity are abstracted, and thirdly that these individual structures culminate in a greater dynamic structure that is ultimately representative of the self.

${ }^{42}$ The parallels between Personal Construct Psychology and qualitative research have also been noted by Butt and Burr (2009). In fact, these authors suggest that because qualitative research is the fastest growing section of work within the British Psychological Society, publicising these parallels will enhance the profile of Personal Construct Psychology amongst psychology academics.
} 
Psychology of Personal Constructs is open to the possibility of elaboration and accepts that his theory is merely a tentative position that is pervious to subsequent development. ${ }^{43}$

\begin{abstract}
A theory should be considered as modifiable and, ultimately expendable...it would mean that we would consider any scientific theory as an eventual candidate for the trash can... Since a theory is an ad interim construction system which is designed to give an optimal anticipation of events, its life is limited by its period of usefulness...when its place is eventually taken by a more comprehensive, a more explicit, a more fertile and more useful theory, it will be time to relegate it to history...the establishment of real fertility in this respect, however, will depend upon what the readers of this manuscript come up with as a result of reading. (pp. 30, 31, 102-103)
\end{abstract}

The theoretical modesty that is inherent within this passage can also be found elsewhere within Kelly’s work. In fact, Kelly (1958/1969) states that, "what we propose, even in its truer aspects, will eventually be overthrown and displaced by something with more truth in it. Indeed, our theory is frankly designed to contribute effectively to its own eventual overthrow and displacement" (p. 66). Thus on the back of this modesty it is argued here that taking greater account of language and its role in being human represents a theoretical approach that is something with more truth in it. Moving the direction of taking greater account of language thereby opens Kelly's (1955) theoretical position to the possibility of the displacement that he ultimately envisioned.

\title{
Chapter Summary
}

This chapter followed the idiographic direction identified at the conclusion of the previous chapter as a productive avenue to pursue in the development of a new theoretical position for qualitative research. What was ultimately sought was a theoretical position that embodied the core principles of the idiographic position and was capable of dealing with the subject matter at a particularly high level of abstraction - such as qualia. Viewed superficially the Psychology of Personal Constructs suitably embodies these requirements. However, the question of whether Kelly (1955) has dealt adequately with language challenges

\footnotetext{
${ }^{43}$ Neimeyer (1985) suggests that Personal Construct Psychology was at risk of what he termed "intellectual isolationism" which he suggests is "the tendency of theory group members to disaffiliate themselves from other traditions of thought, and instead perpetuate a relatively 'ingrown' pattern of communication with one another" (p. 149). In light of this, Fisher $(2003)$, Neimeyer $(1985,1995)$ and Chiari (2000) go one to emphasise the possibilities that elaboration can bring to a revitalisation of Personal Construct Psychology. Moreover, Chiari (2000) suggests that we "pursue the different implications of such and other possible interpretations with regard to those aspects that, even though present in Kelly's original exposition, deserve or require further elaboration" (p. 76).
} 
the theoretical reflexivity of his position as well as the theoretical unpacking of his Fundamental postulate and elaborative corollaries.

Central to this challenge is a view that in order to make the abstractions of similarity and difference that lie at the core of what a construct is; to make something of an event as a reconstrual; to situate our constructs within a greater system of constructs; and to locate our own construct in relation to the reality of the world as well as the constructs of another person, the construct must come to language. In other words, in order for us to engage in the movement of abstraction that Kelly (1955) sets down, a more sophisticated conceptualisation of the inextricability of the dialogue of abstraction, thought and language is required.

Given the productive possibilities envisioned from elaborating Kelly's position for achieving the aims of this thesis, an understanding of the theoretical implications of the way in which he inadequately dealt with language is considered valuable. To ensure that the theoretical position developed by this thesis is able to overcome the challenges identified within Personal Construct Psychology, those challenges must first be examined more closely. This is the task of the following chapter that provides a deeper examination of the implications of Kelly's (1955) inadequate engagement with language and his overlooking of the crucial role of language in the construction of realities and selves. 


\section{CHAPTER 4}

\section{KELLY'S NAÏVE VIEW OF LANGUAGE}

The previous chapter identified the idiographic perspective as a most productive approach for representing a real in qualitative research. Embodying the essential principles of the idiographic approach The Psychology of Personal Constructs was however problematised by the way that Kelly (1955) inadequately dealt with language. This lack of engagement with language opens up to challenge the theoretical reflexivity and subsequent tenability of Personal Construct Psychology. This chapter will begin with a deeper examination of the naïve assumptions about language that can be seen within Kelly's position, as well as providing a closer examination of the theoretical implications for Personal Construct Psychology of Kelly's inadequate account of language. These implications ultimately culminate in a challenge to the claim to a realism that can be made by the Psychology of Personal Constructs, a challenge exemplified here by Noaparast's (1995) notion of Sophisticated Realism. The chapter concludes by suggesting that elaborating Kelly's work in the direction of language provides a productive avenue through which to pursue a hermeneutic re-envisioning of Kelly's (1955) position.

\section{Theoretical Reflexivity}

The previous discussion concerning the notion of theoretical reflexivity has suggested that a theoretical position looking to represent the individual person going about the business of being human must be recognisable as sufficiently commensurate with the actuality of what the business of being human actually is for anyone who stumbles across it (McWilliams, 2004). In other words, a person must be able to see him or her self as represented by way of that theoretical position if it is to be considered a valuable position to pursue. Hence if the principles embedded within Kelly's approach are to be of utility for the new theoretical position being developed, they must be reflexive of being human more broadly. That is, they must embody the way in which a person is able to bring to language his or her 'inner outlook' for the purpose of qualitative research. Thus, on the basis of a suggestion that Kelly (1955) inadequately dealt with language, making his position less theoretically reflexive than it could 
be, it is toward an account of language that this thesis turns in order to develop an elaboration of the Psychology of Personal Constructs. ${ }^{1}$

The following reflections may be helpful in locating the stance that is taken by this thesis regarding the importance of language and for highlighting the ever present potential for language to be assumed to just simply 'be there' as a transparent and unproblematic tool for doing the job of going about the business of being human.

As you, the reader, move through the pages of this thesis it is a journey contingent upon the language written on the page. Similarly as you ponder the argument being made or think to yourself about the mental processes of the author, you are thinking in language, you are using language to negotiate your own way through your day. You may turn to your partner and ask their opinion about this thesis and he or she may offer you some solace for your efforts, all of which is a process founded upon language. Perhaps you are thinking, 'surely, there are however many aspects of life that do not require language?' You would be right, for example a person can make a cup of tea without language. But can you experience it? Or in Kelly's (1955) terms, can you make something of it, without language? Can you make something of the temperature of the tea, experience what it is like to feel the warm smooth surface of the cup within the hand, and the taste of the combination of milk and tea that seems to vary with every cup, without language - absolutely not. Without language the rapid movement of the cup away from your now burnt lips is merely a reflex, the taste is but a texture in your mouth. Until you infuse these events with language you cannot begin to make something of them and therefore experience them. This something that you make of the tea represents the qualia of the tea those internal aspects of the experience for you. It is through and within language that you think, that you experience and

\footnotetext{
${ }^{1}$ An elaboration in this direction and the openness of Kelly (1955) to theoretical elaboration more generally, is alluded to here by Warren (1998), "An elaboration of the sociality and commonality corollaries in the direction of greater account being taken of the social realm (whatever that turns out to be) and the role of language (whatever best account of language emerges from the efforts of linguistics and philosophers of language) appears well accommodated by personal construct psychology. This can be argued specifically in terms of its content, and in terms of its own humility as a theory; that is, its invitation to use it, modify it, and move on" (p. 171). Despite the acknowledgement of Kelly's (1955) invitation to elaborate his work, Warren (1998) provides a warning, "the simplest aspect of this warning is that whatever integrity personal construct psychology might have in respect of the problems of philosophy, it remains nonetheless a psychology. It is concerned with our understanding of human psychic life, not with the traditional questions of philosophy" (p. 121).
} 
that you are able to come to meaningfully bring the events of the world to understanding as you go about the business of being human.

This exemplar is a provocative vehicle through which to begin to apprehend the way that this thesis considers language as the medium within and through which the world assumes meaning. Moreover, it provides recognition of the inextricability of thought from language. That is, referring back to a previous discussion concerning the expressive account of language, we will recall that we do not simply have a thought and then bring that thought into words. $^{2}$ Instead, thought has its condition in language, where language is the essential medium of the 'inner'. The view held by this thesis therefore, is that if language is the medium of the 'inner', then that 'inner' can readily be realised as an 'outlook'. This is because the words that constitute thought as 'inner' are not different from those words that can be said aloud as an 'outlook'. In taking such a position this thesis argues that nothing that can be meaningfully understood is able to get behind or be understood independently of language. Instead language is the first and foremost medium within and through which we go about being human (Gadamer, 1960/2003). Language is that within and through which we live. It is a medium that makes possible and sustains human social life and the possibility of the world coming to meaningful understanding for us (Gadamer, 1960/2003).

It was mentioned in the previous chapter that Kelly's (1955) work is open to challenge upon two fronts. Firstly, that due to his situating of the construct beyond the reach of language, his fundamental postulate and amplifying corollaries are theoretically unsustainable. Secondly, where one person claims to have brought the constructs of another person to language - a claim that is itself theoretically incoherent with Kelly's (1955) own position - this person is accepting that language points toward a thing, in this case the other person's constructs. In so doing, however, one is subscribing to a naïve account of language that suggests that words carry unambiguous and therefore transparent meaning. At the core of such approaches, as indicated in the previous chapter, is a naïvety toward the presence and work of language as a medium. Such approaches overlook the inextricability of language from being and assume that the elements of language are merely ciphers for objects - such as constructs (Gadamer, 1960/2003). Therefore, a specific focus upon the naïve assumptions about language and its subsequent transparency that can be found within Kelly's position, as well as the implications of these assumptions is timely.

\footnotetext{
${ }^{2}$ To review a deeper consideration of the expressive account of language, see a review in Chapter Two.
} 


\section{The Transparency of Language in Kelly's Work}

It has been established previously through Taylor's (1985) model that the nature of language can be pursued according to mutually contradictory accounts of language as either designative or expressive in nature. Recall that Taylor (1985) delineates expressive accounts as providing a view of language as inexhaustible. Here the meaning of an expression can only be explained by yet another expression. In this view language and thought are inextricable, to the extent that language conditions thought. In contrast to this view is the designative account which regards language as a tool that simply points to things, it signifies or stands for something in the world. Such an account subscribes to a position of perfect transparency, whereby the meaning embodied by language is unambiguous and therefore objective. In order to overcome subject-related properties of meaning in the expressive account of language, designative accounts subscribe to a view of language as merely a mnemonic tool for thought. From this perspective thought is somehow wordless or occurs prior to it being brought to language. Central to this view is a suggestion that words are able to simply point to these thoughts and that the meaning of that thought is carried unambiguously or transparently in the words that are uttered. With this brief recapitulation at hand, the implications for the theoretical tenability of Kelly's (1955) position that result from his subscription to or reliance on a designative account of language of some kind can be illuminated further.

At stake here is the way in which the Psychology of Personal Constructs 'skims' over or 'ducks past' ${ }^{3}$ language, assuming it can go directly to a person's mental processes or to thought itself. The example of 'naïve realism' provides a useful vehicle to unpack further this notion of 'ducking' language. ${ }^{4}$ Nä̈ve realism subscribes to an apparent 'common sense' approach to thought. It assumes an entirely unproblematic and direct connection between person and world, devoid of any consideration of the unavoidable intermediary role and therefore work of the human sensorium in perception. In the same manner, in which naïve realists bypass or sidestep the sensorium, those advocating naïve or designative accounts sidestep language. ${ }^{5}$ Thus, suggesting that Kelly (1955) 'ducks' language is to suggest that for him the mental process of thought and language are not one and the same. Instead, the person

\footnotetext{
${ }^{3}$ To 'skims over' or 'duck past' language is terminology first proposed by Leman (1970) in his work Words and Worlds. Within the pages of this text he provides a provocative critique of The Psychology of Personal Constructs with regards to the position and function of language. It is a critique with which this thesis has much in common with.

${ }^{4}$ The debate concerning issues of naive realism is expansive and beyond the scope of this thesis, however for a focused discussion of these debates the following text is recommended: Fish, (2010). Philosophy of perception: a contemporary introduction.

${ }^{5}$ For a detailed philosophical discussion of language's capacity to simply represent thought see: Heil (2004). Philosophy of mind: a contemporary introduction ( $\left.2^{\text {nd }} E d.\right)$.
} 
he envisages is able to have a thought, reflect upon that thought and then unproblematically bring that thought to language after it has occurred. The following passage from Mair (2000) problematises the tenability of such naïve or designative accounts of language and provides an entrée to a deeper consideration of the naïve view of language propounded by and indeed necessitated by Kelly (1955).

At present, we seem mostly to assume that language is virtually transparent, and that we can see through our words and sentences to the things in the world that we are focusing our attention on. Words are still often assumed to be like transparent containers of fairly specific meanings. The speaker puts the meaning he or she intends into the particular words and then sends them across to the listener who unpacks the meanings from the word containers, before selecting other word vehicles to contain the meanings they want to send in response... This whole view is wrong and must be abandoned. Language is now recognized, by those who have been concerned most closely with it, as a very different kettle of fish from this. No longer is it assumed that words and sentences gain their meaning from some direct reflecting of things in the world, but rather from the internal relationships within the language system itself. The relationship of language to reality has become much more questionable. In addition, language has to be regarded as 'substantial', rather than 'transparent'... words and structures in language shape us more than we shape them (p. 342, emphasis in original).

Although Mair (2000) is not forthcoming about the way in which he foresees language's substantiation, he recognises that such a substantiation requires more than mere lip service. Rather, it requires a considered theoretical unpacking of the relationship between language and reality. ${ }^{6}$ It is the substantiation of the relationship between language and reality and more specifically between language and the construct that have been emphasised as lacking in the Psychology of Personal Constructs.

\section{Essential but Incoherent Link between Construct and Language}

Within the Psychology of Personal Constructs and later elaborative work, Kelly is careful to always provide himself a theoretical ladder to get himself out of any subsequent

\footnotetext{
${ }^{6}$ Mair (2000) goes on to suggest that if the disciplines of science unpacked the theoretical indissolubility between language and reality, this may end up challenging the canons of proof that underpin science itself. Indeed, if an expressive account of language was to be adhered to as opposed to language being seen as simply a cipher for objects, then the language of science could become nothing more than a special pleading and persuasion.
} 
theoretical-hole he may find himself slipped into. This wiliness is perhaps epitomised by his consideration of the links between the construct and language. Kelly, in a characteristically pragmatic conceptualisation, situates language as merely a tool (1955, pp. 138, 354, and 1962 , pp. 4, 11, 14) or instrument (Kelly, 1964, p. 148) that can be used to elaborate and transmit meaning. Kelly (1964) presented a paper titled The Language of Hypothesis: Man's Psychological Instrument, ${ }^{7}$ which makes clear this understanding of language.

Even after continued experience in psychotherapy, most of us still hold doggedly to the belief that one man's understanding of the universe can be somehow encoded within a signal system and then transmitted intact to another man via the senses. The signal system is often called "language."...a device he uses to represent his circumstances. (pp. 147, 148)

Here the instrumental nature of language as a series of signals for re-presenting our understanding - as opposed to being seen as the condition of understanding itself - clearly demonstrates what is for Kelly a separation of our mental processes from language. That is, a separation between our construct(s) and language. ${ }^{8}$ This passage clearly overlooks the inherent indeterminacy of language as a medium, and instead firmly situates Kelly's work within the domain of designative and transparent accounts of language.

Nevertheless Kelly's theoretical wiliness makes him difficult to typecast. For instance, several of his clinical tools - the Self-Characterisation and the Repertory Grid Technique - suggest that one person is able to illuminate the constructs of another person. Both of these tools have their basis in the dialogical use of language and therefore the coming into language of a construct. And yet, to return to the essence of the first challenge, Kelly is adamant that the construct has its being beyond the reach of language. ${ }^{9}$ With this disparity in mind a turn is now made toward a close reading of Kelly's (1955) work in order to emphasise the adamancy with which he held this view. Following this, a sketch of what is referred to as the Linguistic Turn within philosophy, will highlight the inextricability of language from

\footnotetext{
${ }^{7}$ The main aim of this presentation was for Kelly to elaborate a position he terms the 'Invitational Mood'. Based upon the 'as if' conceptions from the work of Hans Vaihinger, the 'Invitational Mood' invites a person to adopt a different position with regard to something else. That is, this approach seeks that a person suppose or pretend 'as if' something is something else, and adopt a novel interpretation of the thing and therefore act in a manner accordingly as a form of psychotherapeutic practice.

${ }^{8}$ Proctor (2009) echoes this seprartion of construct and language very clearly, "constructs are not verbal" (p. 23). Instead for him, language is attached to the poles of construct later.

${ }^{9}$ As has been noted previously, Kelly is extremely explicit regarding this point. For instance, Kelly (1958) contends that, "The personal construct...bears no essential relation to grammatical structure, syntax, words, language, or even communication" (p. 87).
} 
being human as well as some of the key theoretical implications for Personal Construct Psychology of Kelly's inadequate dealings with language.

\section{The Construct that is Beyond the Reach of Language}

Arguably two of the most respected scholars within the discipline of personal construct psychology, Chiari and Nuzzo are responsible for a sustained and considered call for Personal Construct Psychology to take a hermeneutic turn. Arguing for a Hermeneutic Constructivism, Chiari and Nuzzo (2004) contend that despite the "crucial importance of language in the construction of realities and selves" (p. 57), the Psychology of Personal Constructs pays limited attention to language. ${ }^{10}$

Kelly does not appear to have adequately dealt with the topic of language.

The...distinction between non-verbal, and verbal constructs, combined with the warning not to confuse a personal construct with its verbal label, appear to be almost the only reference to language in PCT. ${ }^{11}$ (pp. 57-58)

Although this thesis does agree in principle with Chiari and Nuzzo's (2004) point that Kelly (1955) did not adequately deal with language, the thesis also seeks to emphasise that Kelly did make several purposeful references to language. In fact, he is very explicit in detailing that the construct has its being outside of or beyond the reach of language. A consideration of the way in which Kelly (1955) rejects a connection between language and the construct provides a foundation upon which to pursue further the implications of this inadequate consideration of language for Kelly's theoretical position.

Despite Kelly's manifest slippage into naïve or designative assumptions about language, as evidenced by his belief that language is merely a 'system of signals' that transmit the activities of one person's internal mental processes to another, he is adamant that the construct is in fact a mental process that is removed from the reach of that 'signal system'. That is, the construct is proposed to exist - somehow - outside of language. The following

\footnotetext{
${ }^{10}$ Despite the lack of adequacy with which Kelly dealt with language several theorists derive interpretations of Kelly's work as a coherent narrative approach for understanding another person at the level of his or her constructs. Examples of these can be found in the following works: Botella, Corbella, Gomez, Herrero, and Pecheco (2005), Feixas (1995), Botella and Herrero (2000), Mair (1988, 1989, 2000), Neimeyer (1994, 2000, 2001, 2009), Neimeyer and Stewart (2000) and Viney (1993). The need for elaboration in the direction of language is also recognised by Warren (1998), arguably the most prolific and respected contributor to a consideration of the philosophical domain of the Psychology of Personal Constructs, who calls for "an elaboration of the sociality and commonality corollaries in the direction of greater account of...the social realm... and language" (p. 171).

${ }^{11}$ PCT or Personal Construct Theory is a commonly used abbreviation within the writing concerning the Psychology of Personal Constructs to recognise that consideration is on theoretical rather than practical aspects of the work.
} 
points, drawn from a close reading of Kelly's work, underscore the degree of adamancy with which he espouses this separation between language and the construct. So much so, that his position ultimately suggests that bringing a person's constructs to language is in fact a human impossibility.

Since it [the construct] is a psychological affair, it has no necessary allegiance to verbal forms in which classical concepts have been traditionally cast. The personal construct we talk about bears no essential relation to grammatical structure, syntax, words, language, or even communication...it is simply a psychologically constructed unit for understanding human processes. (Kelly, 1958, p. 87, emphasis added)

Some therapists decry "acting out" on the part of the client. They want the client to verbalize all of his constructs. It would be nice if the client could. But he cannot. (Kelly, 1955, p. 464, emphasis added)

Here is a clear and unequivocal statement of Kelly's conceptualisation of the construct as a thing that has its essential being beyond the grasp or even scope of language. Kelly also makes several references to the impossibility of any full conceptualisation of the construct in language throughout his work. In fact, at times he is explicit that not only are our constructs unable to be brought into language, ${ }^{12}$ but also that our constructs are something different from language all together.

Let us give the name constructs to these patterns that are tentatively tried on for size. They are ways of construing the world. They are what enables man, and lower animals too, to chart a course of behavior...verbally expressed or utterly inarticulate. (Kelly, 1955, p. 9, emphasis added)

Our view of constructs does not limit them to those which are symbolized by words, or even to those which can be communicated by means of pantomime. Perhaps the psychology of personal constructs is an intellectualized theory. But if, by intellectual controls, one means that the constructs are communicated, then there are some kinds of controls which are not intellectual, since they are not communicated. A large portion of human behavior follows nameless channels which have no language symbols, nor any kinds of signposts whatsoever. Yet they are channels and

\footnotetext{
${ }^{12}$ This is a clear example of the 'Beetles' that Wittgenstein (1953/1968) used as exemplars in his 'Private Language argument'. This will be explored in more depth later this chapter.
} 
they are included in the network of dichotomous dimensions with relation to which the person's world is structured... While the psychology of personal constructs is concerned with personal constructs all of which may not be communicable, and hence is not really what some would call intellectualized theory, it is important that it be itself communicated and that it be intellectually comprehensible. Here we distinguish between the personal constructs about which the theory is concerned and the constructs which constitute the approach of the theory itself. The former may or may not be communicated; the latter must be communicated to make public sense.

(Kelly, 1955, p. 130, emphasis added)

Now these abstractions are not necessarily verbalized by him, nor are they necessarily immediately translatable by him into verbalizations, either in the public language or in his own babble. His abstractions of his own behavior may be structured or construed by him solely in terms of anticipated continuities and cycles. They are still abstractions. They are isolated. There is still a construct-like discrimination of simultaneous likeness and difference in the way he thinks about them. (1955, p. 173, emphasis added)

The first statement - consistent with the designative account of language - emphasises the primacy of the construct for going about the business of being human and yet also underscores its inherent separation from language. When read in conjunction with the second passage of Kelly's work, it becomes evident that the construct is in fact resistant to representation in language. Here, then, Kelly is clear that the construct is considered to be something that is other, as something that exists outside of language. Although he gives the impression that a person may indeed 'bump into' these structures, he is consistent that he or she will find it difficult to bring these constructs into the public language or even what Kelly terms an individual's 'own babble'. ${ }^{13}$ The inherent separation between construct and language is here again writ large.

Recall that Kelly suggested that a person's psychological process followed networks of channels formed by his or her constructions. ${ }^{14}$ In combination these channels form a

\footnotetext{
${ }^{13}$ In making this statement Kelly is suggesting the possibility that an individual bump into their constructs in a language developed by and for one's own use, a private language. A subscription to the possibility of Wittgenstein's (1953/1968) Private Language opens up to challenge the tenable nature of Kelly's theoretical position. A more focused discussion of the implications of Kelly's suggestion that a private language is a possibility will be undertaken later this chapter in a discussion of the Linguistic Turn.

${ }^{14}$ For a discussion on these channels see a review of this notion in Chapter Three.
} 
person's greater system of constructs which comes to represent for Kelly the Self, the embodiment of all that a person can make of the world. It follows therefore that these channels - and therefore by association the Self-cannot be externalised in language. ${ }^{15}$ The following passages further support this reading of Kelly's (1955) work.

Construing is not be confounded with verbal formulation. A person's behavior may be based upon many interlocking equivalence-difference patterns which are never communicated in symbolic speech. (Kelly, 1955, p. 51)

It may be that the constructs which are most important in a client's life have no kind of symbolization at all, either verbal or nonverbal. (Kelly, 1955, p. 198, emphasis added)

It is not easy to find words and sentences to express the deeply rooted constructs by which the self is fixed into place. (Kelly, 1955, p. 334, emphasis added)

At the core of these statements is a clear suggestion that a person cannot be articulate about matters of the Self. Worded another way, the person that Kelly (1955) envisages is unable to be articulate about the matters of most importance to them. This rests of course on Kelly situating the construct as a thing that has its being outside of language, and that therefore cannot be articulated. ${ }^{16}$ The following passages further exemplify Kelly's view.

It is not possible for one to express the whole of his construction system. Many of one's constructs have no symbols to be used as convenient word handles. They are therefore difficult, not only for others to grasp and subsume within their own systems, but also difficult for the person himself to manipulate or to subsume within the verbally labeled parts of his system. The fact that they do not readily lend themselves to organization within the verbally labeled parts of the system makes it difficult for a person to be very articulate about how he feels, or for him to predict what he will do in a future situation which, as yet, exists only in terms of verbal descriptions. (Kelly, 1955 , p. 110, emphasis added)

\footnotetext{
${ }^{15}$ The way in which Kelly himself situates an individual construct - and therefore a person's greater system of constructs - beyond the reach of language has significant implications for his claim to a realism. These theoretical implications will be explored later in this chapter.

${ }^{16}$ This separation of the construct from language is underscored by Proctor (2009) who suggests, "constructs are not verbal. Words or 'signifiers', later become attached to the poles of constructs and we clearly later learn thousands of constructs via the language in which we are immersed" (p. 23).
} 
We recognize that the psychological notion of construing has a wide range of convenience, which is by no means limited to those experiences which people can talk about or those which they can think about privately. (Kelly, 1955, p. 51)

These passages from Kelly's (1955) work clearly identify a theoretical disconnect between language, construct and thought. Here the person that Kelly conceives of is unable to bring into words the way that the world is for that person. ${ }^{17}$ As well as taking the view that such an approach is not theoretically reflexive - in that a person is often required and able to be articulate about their inner emotions and feelings - this thesis also contends that Kelly's view of the person is theoretically incoherent and therefore unsustainable. Take for example Kelly's (1955) introduction to the process of self-characterisation. Despite the way that he has argued that the construct is beyond language and that the person he envisages cannot be articulate about his or her feelings, he goes on to suggest.

If you do not know what is wrong with a person, ask him; he may tell you. The clinician will have to be prepared to do some careful listening, for the answers will be couched in terms of the respondent's personal constructs. (1955, pp. 322-323)

This passage is unequivocal that for Kelly the construct(s) and a person's inner feelings can indeed be brought to language, highlighting a significant theoretical discordance within his work. On the one hand, he is resolute that the construct does not lend itself to being brought to language, and yet here he suggests otherwise. A conundrum indeed, perhaps made all the more complex by statements such as these:

These are likely to refer to constructs which not only have a wide range of convenience for the client but also which he cannot be sure he is able to communicate by mere words. (Kelly, 1955, p. 333, emphasis added)

Words, when used as symbols, and they often are so used, are not the constructs they represent; they are representative contextual elements of those constructs. (Kelly, 1955, p. 200, emphasis added)

\footnotetext{
${ }^{17}$ Despite Kelly's (1955) claim to the contrary, Fry, Butt and Bell (2003), using a modified version of a Repertory Grid technique outlined by Butt, Burr and Bell (1997), suggest that "people certainly have a sense of self that pervades their interaction" (p.71), and are able to be articulate about themselves at the level of core structures that make up the Self. Here a clear disconnect can be seen between Kelly's (1955) theoretical position and the work of these authors.
} 
Although these passages are consistent with the previous discussion - where Kelly explicitly suggests that the construct is such a high level of abstraction that it is beyond the reach of language - he goes on to emphasise that those aspects of a person's construction system that he or she $i s$ able to bring into words are not constructs themselves. Rather, it is the elements within the construct that he or she is able to bring to language. ${ }^{18}$ In order to develop an abstraction that then stands for the construct, a clinical practitioner or someone wishing to apprehend another person's constructs is surely obligated to subscribe to a naïve, designative or transparent view of language. Worded another way, the clinician must develop an abstraction of a construct, as an 'abstraction of an abstraction'. More specifically, an abstraction of a thing that supposedly cannot come to language. On the topic of words as elements the following passage from Chiari and Nuzzo (2010) is valuable,

In Kelly's conceptualisation, constructs are usually symbolised by invoking one of their elements. The elements stand not only for itself, but also for the whole construct. The introduction of a word in the construct context usually as one of the 'like' elements - allows the person to use it as a symbol. This 'trick' makes the revision of constructs relatively feasible. (p. 116)

However, consistent with the work of Kelly (1955) who states, "Even the elements which are construed may have no verbal handles by which they can be manipulated and the person finds himself responding to them with speechless impulse" (p. 16), Chiari and Nuzzo (2010) go on to suggest - in the paragraph that proceeds the passage above - a clear incompatibility between language and the elements of the construct.

The lack of word symbol makes it difficult for people to communicate their constructs or their elements, and for the therapist to understand their clients. The clients can act out their constructions, or just sit and have feelings they cannot describe. The therapist will have to infer the client's constructions, and eventually make recourse to role playing and other 'non-intellectual' approaches. (p. 116)

In taking such a position, these scholars emphasise an untenable relationship between language, the construct, and its elements. This position supports the theoretically problematic nature of Kelly's position in so far as locating the construct beyond the reach of language

\footnotetext{
${ }^{18}$ On the topic of words and elements Proctor (2011) suggests, "words may be included alongside other elements and these are used to communicate the meaning of the construct, more or less successfully" (p. 44). It is important to appreciate that it is not the construct that is coming to language here, but merely those elements that are able to come to language.
} 
clearly suggests that the task of bringing a single construct, let alone an entire construct system, to language is an absolute impossibility. This opens Personal Construct Psychology up to questions concerning the degree of reflexivity that is genuinely embodied by the position, as well as its overall theoretical tenability. More specifically, Kelly's depiction of language will be shown here to have widespread implications for the Psychology of Personal Constructs' claim to a realism.

At this stage, then, in order to elucidate the full weight of these theoretical implications, further support for the argument that Kelly's theoretical position is untenable needs to be provided. Much philosophical groundwork has already been undertaken in other disciplines to suggest that language discloses the world. This work provides a further and robust consideration of the theoretically untenable nature of approaches that adopt naïve assumptions about language in the way that Kelly's (1955) does. As such we move now toward a consideration of the significant philosophical movement referred to as The Linguistic Turn.

\section{The Linguistic Turn}

The linguistic turn accepts as its starting point that language is not a transparent instrument of thought where words function like labels simply attached to concepts - or in this instance constructs. Rather, the linguistic turn embodies most completely the view that language constitutes reality. Proponents of the linguistic turn - for instance, Gadamer (1960), Herder (1772/2002), Humbolt (1988), Hamann (1949-1957, 1967), Heidegger (1926/2005) Rorty (1967), Russell (1985), and Wittgenstein (1968) - subscribe to what has been described previously as an expressive account of language, where the meaning of an expression cannot be understood in the absence of other expressions. This view highlights the inherent indeterminateness of language that is at the core of expressive accounts. Ultimately expressive accounts hold that anything outside of language is inconceivable given that it has no meaning. This is because in an expressive account there is no separation between the world as an object and the way in which it comes to meaning in language. Specifically language is the medium that discloses a world. From the perspective of such a position, a person's processes of mental abstraction and language are inextricable and therefore so too are the Self and language, or thought and language. ${ }^{19}$ To elaborate further the core elements

\footnotetext{
${ }^{19}$ Theorists who align themselves with the tenets of the 'linguistic turn' (cf. Gadamer, Herder, Humboldt, Hamann, Rorty, Wittgenstein) subscribe to a view that language and thought are inextricable. From this perspective then, the gulf that has been established between mind, world and language by 'analytical philosophy' represents a chimerical and illusory impossibility.
} 
that are embodied by the Linguistic Turn, the work of Wittgenstein - who can be thought of as having made his own linguistic turn - will now be considered.

Wittgenstein's early work, the Tractatus Logico-Philosophicus (1922), ${ }^{20}$ proposes that language is the basis of proposition and that propositions provide pictures of the world. In the preface to this work, Wittgenstein $(1922 / 1999)$ provides the following passage.

What can be said at all can be said clearly; and whereof one cannot speak thereof one must be silent. The book will, therefore, draw a limit to thinking, or rather - not to thinking, but to the expression of thoughts; for, in order to draw a limit to thinking we should have to be able to think both sides of this limit (we should therefore have to be able to think what cannot be thought). The limit can, therefore, only be drawn in language and what lies on the other side of the limit will be simply nonsense. (p. 27)

The Tractatus is a consideration of the nature of language and more specifically the nature of language as adapted to the representation of facts. Within its pages, Wittgenstein introduces the notion of a proposition, which for him expresses a fact. Therefore propositions must fit with the facts they are designed to express. For this purpose Wittgenstein proposes a picture approach to meaning. Here words provide a picture of possible states of affairs, and in so doing provide what is considered to be a potential correspondence between these pictures and the world. Thus, for Wittgenstein in the Tractatus (1922/1999), this is the way that language "touches reality" (p. 34). At the heart of Wittgenstein's thought in the Tractatus is the contention that a word as a name is in fact meaningful because there is an object that it signifies. On the basis of this, Harre and Tissaw (2005) comment that when it comes to discerning the truth of what is said, "the Tractatus eliminates 'the psychological'... no wonder it is difficult to find a thinking individual in the Tractatus" (p. 45). ${ }^{21}$

The theoretical unpacking of the Tractatus is not of course the principal remit of this thesis, but it does provide a foundation to highlight the significance of later theoretical moves Wittgenstein himself undertook. With the posthumous publication of Wittgenstein's

\footnotetext{
${ }^{20}$ Tractatus Logico-Philosophicus (1922) is the only work published during Wittgenstein's lifetime. His later work Philosophical Investigations (1953) - arguably his most noteworthy contribution to contemporary philosophy and the Linguistic Turn - was published posthumously.

${ }^{21}$ In the Tractatus, Wittgenstein (1922/1999) suggested a correspondence between the structure of the sentence and the structure of reality as a correspondence between signifier and signified. In so doing, Wittgenstein (1922/1999) eliminates the psychological or contextual aspects of an utterance. It is on the basis of his eliminating of the possibility that words can carry meaning that goes beyond simply what is said that Harre and Tissaw (2005) suggest that we will not find a thinking person within the Tractatus.
} 
Philosophical Investigations, we can witness a theoretical movement that epitomises similar shifts to those made within the Linguistic Turn more broadly. As mentioned in the Tractatus, Wittgenstein argued then that the word is a name that operates to signify an object as a proposition. $^{22}$ In stark contrast, within Philosophical Investigations he suggests that the notion of the proposition be replaced by the idea that sentences have meaning by virtue of the circumstances within which they are uttered and the language game to which they belong (Harre \& Tissaw, 2005; Kenny, 2006a, 2006b; Wittgenstein, 1953/1968). ${ }^{23}$ Of most importance to this thesis, however, is Wittgenstein's (1968) unpacking of his 'private language argument'. The central characteristics of this argument provide a useful vehicle for exploring some of the problematic implications resulting from Kelly's (1955) depiction of language. ${ }^{24}$ The core of Wittgenstein's (1968) argument is that a language that is understandable to only one individual person is in fact incoherent. In fact, following this argument to its conclusion, he highlights that those things that we can say nothing about - for instance our constructs - are of little use. A closer examination of the Private Language argument is explanatory of Wittgenstein's conclusion.

\section{The Private Language Argument}

The idea of a private language concerns the development, or the possibility of developing, a private or personal language for our private feelings, thoughts and so on. Beginning with Wittgenstein's (1968) consideration of this proposal is a valuable introduction.

But could we also imagine a language in which a person could write down or give vocal expression to his inner experiences - his feelings, moods, and the rest - for his private use? - Well, can't we do so in our ordinary language? But that is not what I mean. The individual words of this language are to refer to what can only be known to the person speaking; to his immediate private sensations. So another person cannot understand the language. (pp. 88-89)

\footnotetext{
${ }^{22}$ Kenny (2006a) described the designative capacities of the name in terms of 'metaphysical atomism'.

${ }^{23}$ It is important to appreciate what it is that Wittgenstein understands by the notion of a 'language game'. Although scholars debate its meaning, the term 'game' draws inspiration from the games children play, some with a series of complex rules and some with very few. All games, however, can be considered to have some degree of commonality, even if only for the purpose of playing the game. Importantly Wittgenstein is not attempting here to trivialise language, but rather to indicate that language has a context and is a communal activity that we get caught-up in, in much the same way as we get drawn along by the movement of a game (Kenny, 2006a).

${ }^{24}$ For a most accessible and complete discussion of Wittgenstein's Private Language Argument see: Harre and Tissaw (2005). Wittgenstein and psychology: a practical guide.
} 
Here Wittgenstein (1968), to use other words, suggests that “an 'inner process' stands in need of outward criteria" (p. 153). With recourse, then, to the theoretically reflexive position outlined previously, it is undeniably evident from the everyday business of being human that we can indeed express how we feel, and understand how others people feel by way of a diverse and refined vocabulary. Thus, if a theoretical position is to be genuinely reflexive, then it should encompass the way in which we can indeed be articulate about our inner feelings and processes. However, with regard to the previous elaboration of the Psychology of Personal Constructs, it is clear that by situating the construct beyond the reach of language, Kelly shut down the possibility of there being outward criteria by way of which a person can bring forward his or her 'inner processes' in language as an 'outlook'. Certainly Kelly at times contradicts himself, suggesting that a person could bring aspects of his or her construct system to understanding - albeit in his or her own babble. However, the fact that the person that Kelly (1955) envisages can bring aspects of his or her construct system to understanding, and yet the construct itself is nonetheless devoid of outward criteria, poses a theoretical and logical quandary. A consideration of Wittgenstein's (1968) 'beetle in the box' analogy provides a useful means of unpacking the complex nature of this quandary. Wittgenstein (1968) sets up the argument that informs this analogy in the following way.

Suppose everyone has a box with something in it: we will call it a 'beetle'. No one can look into anyone else's box, and everyone says that he knows what a beetle is only by looking at his beetle. Here it would be quite possible for everyone to have something different in his box. One may even imagine such a thing constantly changing. But suppose the word 'beetle' had a use in these people's language? If so it would not be used as the name of a thing. The thing in the box has no place in the language-game at all; not even as a something: for the box might even be empty. (p. 100)

In this analogy Wittgenstein is trying to emphasise that the beetle is much like a person's mind. No one can know exactly what it is like to be another person or to experience things from the perspective of another person. In other words, one person cannot look inside another person's box in order to know exactly what is in it. It is generally assumed, however, that every person's mental workings are very similar given that we all have a mind, or in Wittgenstein's terms a beetle. But given that the word 'beetle' - if it is to have any sense of meaning - means simply 'what is in the box', then there is no way or purpose of checking or comparing beetles. Worded another way, the word 'beetle' could not be the name of a thing that can be used in a language game to designate something, because each person may have something completely different in his or her box or may even have nothing at all. Thus, the 
word 'beetle' does little to express what it is that is inside it. For Wittgenstein (1968), then, the beetle, as a purely private object, "drops out of consideration as irrelevant" (p. 293), it cannot be coherently articulated. Wittgenstein argues therefore that although we cannot know what it is like to be someone else, to suggest that there is a special mental entity we call the mind that makes experience private is wrong. He goes on to suggest that a person's inner feelings are not mental entities known only to those who have them - as in the beetle in the box or a person's constructs - instead, having the feeling and having a tendency to a certain expression in public language are internally related as part of the same psychological complex. ${ }^{25}$ Wittgenstein culminates the 'private language argument' by suggesting that if something can be said about a person's state of mind, then it is not 'private' in the sense elaborated. Equally, if it is 'private' then one cannot talk about it. From this argument Wittgenstein (1968) achieves an intimacy of language and our 'inner' mental processes.

What is hidden...is of no interest to us...A nothing would serve just as well as a something about which nothing can be said...the paradox disappears only if we make a radical break with the idea that language always functions in one way, always serves the same purpose: to convey thoughts. (pp. 50, 102)

Having argued against the possibility of a person's mental processes being only representable to the person with privileged or private access to them, and having demonstrated the nonsensical nature of approaches that hold the existence of such a position, Wittgenstein's (1968) Philosophical Investigations looks towards the overcoming of this paradox.

Wittgenstein (1968) therefore challenges the notion that we simply have a thought that is then brought to language, by establishing an intimacy between thought and language; "When I think in language, there aren't meanings going through my mind in addition to the verbal expressions: the language is itself the vehicle of thought" (p. 107). To paraphrase Wittgenstein in terms of the challenges being levelled at Kelly (1955), 'when we think in language there are not constructs going through my mind in addition to the verbal expressions: the language is itself the vehicle of the construct, of thought'. Therefore, with recourse to Wittgenstein's argument, to have a construct outside of language is nonsensical and is of little concern. Although Wittgenstein's work is lauded as 'breaking the mould' of philosophical thought on language, it is suggested that his work fails to elaborate the full extent of the expressivist possibilities opened up through Philosophical Investigations (Lawn,

\footnotetext{
${ }^{25}$ Wittgenstein's (1968) argument also suggests that we cannot know the experience of another person simply by means of generalising from our own experience. He asks, "how can I generalize the one case so irresponsibly" (p. 293). Harre and Tissaw (2005) suggest that these ideas represent a challenge to the belief that "the private feelings of all those people who display similar expressive performances are alike in relevant ways" (p. 190).
} 
2004). ${ }^{26}$ It is for this reason that Lawn (2004) suggests that Wittgenstein's greatest failure was in not making 'hermeneutic turn' and seeing the centrality of interpretation. ${ }^{27}$

\section{Kelly's Private Language}

Central to this discussion of a 'private language' are the consequences both epistemologically and philosophically for conceptions of mind. Several traditional and influential theoretical positions subscribe to a private language as a given. This thesis suggests that Kelly's (1955) work is in fact one example of such a theoretical position. The way in which Kelly (1955) can be seen as succumbing to a private language is captured succinctly by the words of Kenny. Kenny's (2006a) argument suggests that positions that envision the possibility that a person's mind can classify and recognise its own thoughts and experience, while at the same time holding in suspense the existence of the external world and the minds of other people, subscribe to a Cartesian conception that "seems to entail the possibility of a private language or of something very like one" (p. 13). It follows therefore that if private language is found to be difficult to sustain then so too is Kelly's (1955) position. The following discussion outlines the way that Kelly's (1955) position necessitates a subscription to a private language.

It has been highlighted in the previous discussion of Kelly's Fundamental Postulate and his eleven elaborative corollaries that many of his theoretical manoeuvres are untenable given the way in which Kelly situated the construct beyond the reach of language. This is because in order for us to develop abstractions of similarity and difference - to make something of an event as a reconstrual, to hierarchically situate our constructs within a greater system of constructs, or to locate or abstract our constructs in relation to reality itself and the constructs of another person - we must have a medium within which this transpires. That is, the construct must have its being in language. However, by clearly denying this link between the construct and language there is no alternative for Kelly but to subscribe to a position which Kenny (2006a) alludes to as 'something very much like a private language', a position through which a person can classify and recognise their own processes while holding in suspense the external world. ${ }^{28}$ In fact this position is supported by Raskin (2011) who suggests that constructivist approaches such as Kelly's (1955) focus upon individual meaning making processes. He goes on to argue, "they [constructivst approaches] do so because for

\footnotetext{
${ }^{26}$ Not all scholars agree with the label of Genius for Wittgenstein and an engaging critique of his work can be found in: Cohen (2005). Wittgenstein's Beetle and other classic thought experiments.

${ }^{27}$ It is a 'hermeneutic turn' that will be taken by this thesis in Chapter Five.

${ }^{28}$ This position is supported by Raskin (2011) who suggests that constructivist approaches such as Kelly's (1955) focus on individual meaning making processes. "They do so because for constructivists meaning is private and personal" (p.225).
} 
constructivists meaning is private and personal. The individual, as an informaltionally closed system, is only in touch with his or her own processes" (p. 225). Kenny (2006a) discusses the implications for theoretical positions beholden to such views in the following passage.

Some empiricist philosophers have thought that the only matters of fact we really know are our own experiences: what we claim to know about the world or about other people is based on our knowledge of our own mental states and processes. The same philosophers have commonly taken for granted that our knowledge of our experience can be expressed in language, at least to ourselves, and that the possibility of this expression does not presuppose any acquaintance with the external worlds of our minds. Anyone who accepts this much must believe in the possibility of a private language whose words acquire meaning simply by being linked to private experiences. Indeed he must believe that our actual language is a private language, not in the sense that it is peculiar to a single user, but in the sense that its words have acquired their meaning for each of us by an essentially private process: an internal ostensive definition in which an appropriate sample of experience was attended to and associated with a word. Of course, if words are thought of as acquiring meaning in this way, a doubt may arise whether the samples from which one person has acquired his vocabulary are really like the samples from which another person has done so. Thus this form of empiricism carries with it a version of skepticism that finds expression in such thoughts as the following: "For all we know, what I call "red" you call "green" and vice versa' (cf. BB 60; PI, I, 272). ${ }^{29}$ (p. 142)

In light of the previous discussion and Kenny's (2006a) argument as formulated above, this thesis takes the view that despite Kelly's theorising to the contrary there is in fact no possibility of conceiving of the processes of abstraction that Kelly suggests - in his Fundamental Postulate and elaborative corollaries - as being beyond the reach of language. In other words, language must be the medium through which a person engages in the movement of abstraction that Kelly (1955) suggests. However, given the degree to which Kelly is adamant that the construct is beyond the reach of language, he must therefore subscribe to the position outlined here by Kenny (2006a), "that our knowledge of our experience can be expressed in language, at least to ourselves, and that the possibility of this

\footnotetext{
${ }^{29}$ Kenny indicates that it was through his conferring with Wittgenstein's (1958) Blue and Brown Books (p. 60), and paragraph number 272 in Philosophical Investigations (1953/1968) that he derived this statement.
} 
expression does not presuppose any acquaintance with the external worlds of our minds" (p.142). Thus, on the basis of this argument, the Psychology of Personal Constructs must be situated in one of two ways. Either, Kelly's approach assumes the possibility that a person is able to develop a private language with which to undertake the movement of abstraction and in so doing succumbs to the challenge outlined by Wittgenstein's $(1953 / 1968)$ 'private language argument' - or Kelly's approach is theoretically untenable.

\section{Taking Language More Seriously}

This thesis has contended that Kelly (1955) strongly maintained that the construct is a structure beyond the reach of language. In so doing, he situated the Psychology of Personal Constructs as a theoretically untenable position. Here a considered sketch of the way in which other scholars also recognise the need for Kelly to take more seriously the notion of language in dealing with thought and the world outside of thought will be presented (cf. Duck, 1983; Leman, 1970; Mair, 1989, 1990; Noaparast, 2000; Yorke, 1989). In conjunction with the arguments levelled above the work of these authors will support a consideration of the broader implications of Kelly's inadequate depiction of language for the Psychology of Personal Constructs. Identifying what these implications are in turn provides a basis for developing a redress of these implications by the theoretical approach being developed by this thesis for qualitative research.

It is the work of Leman (1970) and Solas $(1992,1995)$ who provide the most sustained and surprisingly uncontested discourse concerning the way in which Kelly dealt with language. ${ }^{30}$ However, Noaparast (2000) - as a proponent of Personal Construct Psychology - provides an unlikely ally in the argument being established here and a useful position from which to begin further discussion.

\footnotetext{
${ }^{30}$ Although these works offer a focused consideration of the way Kelly dealt inadequately with language, Goodrich (1993) in a brief paper titled 'Deconstructing constructs: pitfalls in personal construct theory' is also supportive of these claims. Goodrich (1993) discloses what he believes to be the crucial pitfalls in Kelly's personal construct theory and arrives at the following question, "we have already found this thesis [Kelly's] to be questionable in the case of evnts. Is it also open to serious doubt in the case of language? Is language a consequence of constructs or are constructs a consequence, even in part, of language?" (p.80). Goodrich (1993) goes on to argue that contrary to Kelly's refusal to identify constructs with what he calls verbal formulations, "language, not unlike event [in a Kellian sense], assumes a presuppositional role" (p.81). He concludes his argument by suggesting that Kelly fails to "indicate any awareness of the fundamental, presuppositional standing language bears in relation to our constructs" (p.81). For this author the issue of language ultimately raises doubts about how a person places an interpretation in the process of construing that Kelly elaborates.
} 


\section{Language as a form of Elegant Reductionism}

In an article titled 'Constructs and Words', Noaparast (2000) suggests that "what is doubtful is the reductive claim that all constructs have linguistic nature" (p. 69). This statement provides a suitable entrée to a discussion concerning ambiguity in the way that Kelly (1955) conceptualised the relationship between language and the construct and the process of abstraction. To highlight the nature of this ambiguity, Noaparast (2000) directs attention to the following passage within Kelly's (1955) work.

A construct in turn, is an abstraction. By that we mean it is a property attributed to several events, by means of which they can be differentiated into two homogenous groups. The invention of such a property is the act of abstracting. To construe events is to use this convenient trick of abstracting them in order to make sense out of them. Each person goes at it his own way, more or less, and that is where the title of the book, The Psychology of Personal Constructs, comes from. (p. 120)

In order to apprehend further the ambiguity within this statement, a sketch of the way Kelly (1955) sought to differentiate the construct from the more common notion of the concept is valuable.

We use the term construct in a manner which is somewhat parallel to the common usage of the 'concept'. However, if one attempts to translate our construct into the more familiar term 'concept', he may find some confusion. We have included, as indeed some recent users of the term 'concept' have done, the more concretistic concepts which nineteenth-century psychologists would have insisted upon calling 'percepts'. The notion of a 'percept' has always carried the idea of its being a personal act - in that sense, our construct is in the tradition of 'percepts'. But we also see our construct as involving abstraction - in that sense out construct bears a resemblance to the traditional usage of the 'concept'... Now when we assume that the construct is basically dichotomous, that it includes percepts, and that it is a better term for our purposes than the term 'concept', we are not quarreling with those who would use it otherwise. Within some systems of logic the notion contrast as something distinct from irrelevancy is not part of the assumptive structure. We on the other hand, are simply assuming that this is the way people do, in fact think. (pp. 69-70) 
It is evident that on the one hand Kelly seeks to differentiate the construct as something different from a concept, and on the other he wishes to keep the features of abstraction that the concept embodies. When considered in light of the argument that has gone before, it is evident that Kelly's (1955) inadequate account of language is central to the ambiguity that Noaparast (2000) is alluding to. Thus, while Kelly (1955) has made it clear that the construct is beyond the reach of language, the notion of abstraction is essential to the nature of the construct. ${ }^{31}$ However, it is the abstractive features of the concept that Kelly (1955) wanted to maintain, a feature that is only possible in language. Here the ambiguity that Noaparast (2000) envisaged is palpable and is captured succinctly by way of his following question: "Could it be possible to talk of 'mak[ing] sense out of' events by making abstractions without concepts being involved? In other words, could one talk of meaning without using language?" (p. 66).

Drawing out some of the broader theoretical implications of the way in which Kelly dealt with language and prefacing the arguments outlined within this thesis, Noaparast (2000) goes on to emphasise the requirement of conceptualisation, if indeed the construct system is to exist as a system of subordinate and superordinate relationships. ${ }^{32}$

Kelly's view is that he considers the construct system as ordered by a subsumptive relationship between subordinate and superordinate constructs.

This indicates that a logical relationship is involved in construct systems.

Subsumption is necessarily conceptual, and hence, is not possible without conceptualization. (p. 66)

Here Noaparast (2000) can be seen to support the argument levelled by this thesis. That is, without a construct having recourse to language, the establishment and maintenance of a person's greater system of constructs is untenable. Noaparast (2000) does however concede that "it seems important to concur with Kelly that constructs are not necessarily conceptual and expressed in language" (p. 68). In this case, the nature of the construct - when beyond the reach of words - may be, according to Noaparast (2000), "simply a 'discrimination'

\footnotetext{
${ }^{31}$ The importance of abstraction to the construct is supported by Fransella (1989) who suggestes that concepts and constructs are not dissimilar because both are concerned with similarity between things, and that both involve the notion of abstraction.

${ }^{32}$ The Organization Corollary has established that it is indeed a relationship of subsumption and superordination that Kelly had in mind. We must recall that the Modulation and Fragmentation Corollaries have suggested that the construct system, although not a 'logic-tight' system, does have a degree of internal inconsistency as a function of the permeability of the constructs themselves. Thus, the notion that a construct be considered as a conceptualisation - a position that is most certainly untenable without language - is commensurate with the broader functioning of the construct system, while being simultaneously incommensurate with Kelly's own consideration of the links between language and the construct.
} 
imposed upon events that might be due to conditioning, without any verbalization involved" (pp. 66-67).

Noaparast (2000) also goes on to recall the work of Crockett (1982) who - drawing upon the notion of 'Implicative Dilemma' as outlined by Hinkle $(1965)^{33}$ - indicates that words should not be confused for constructs given that words can carry different meanings in different contexts and, therefore, cannot be used to indicate a construct. ${ }^{34}$ However Noaparast (2000) insightfully suggests that a construct system without any signposts or means of symbolising a construct has problematic implications for Kelly's theoretical position. More specifically, Noaparast (2000) suggests that without language or 'sign posts' or 'word handles' with which to deal with our constructs, we cannot feasibly grasp a construct in order to try it on for fit, or for that matter to choose amongst alternatives. The following passage emphasises Noaparast's (2000) position.

Constructs, here, are to be related to behavior in a causal manner on the ground that where the constructs are not symbolized in 'any kinds of signposts whatsoever', the person can not handle his constructs to deal with them as tentative hypotheses. This is because symbolisation is necessary for any kind of 'handling' of mental components, as is shown by Piaget (1967). And if no handling, then no 'alternative' constructs are available for the person to 'choose'. (p. 68)

Noaparast (2000) here challenges the possibility of what Kelly (1955) has described as "slicing off chunks of time and reality and holding them up for inspection one at a time" (p. 120). However, Noaparast (2000) does stop short of developing further the role of language within Kelly's work for fear of trapping the Psychology of Personal Constructs in what he considers an elegant form of reductionism.

While the linguistic turn is, in a sense, a reflection against positivistic reductionism, an elegant type of reductionism is still associated with most of the new trend in the linguistic turn: reducing thought or psychological processes to language. (p. 68)

\footnotetext{
${ }^{33}$ A closer examination of Hinkle's (1965) notion of 'implicative dilemma' (p. 19) can be seen in his unpublished $\mathrm{PhD}$ thesis titled The Change of Personal Constructs from the Viewpoint of a Theory of Construct Implications.

${ }^{34}$ It must be mentioned here that for Kelly (1955) our constructs are also contextually based. That is, each construct is limited by its range of convenience.
} 
Although Noaparast (2000) is fearful of what he suggests to be the reductive capacities of language, he does go as far as to suggest that "to say language is neither necessary nor sufficient for constructs is not to deny that language plays an important role in constituting construct systems" (p. 69). Here Noaparast's (2000) work can be seen to offer some clear synergies with the arguments established by this thesis. If the construct has its being beyond language - as Kelly (1955) goes to great lengths to establish - then a theoretically untenable position results. If there is no medium through which a person can grasp his or her constructs then the movement of abstraction and therefore reconstrual is incomprehensible. On the other hand, if language does play an important role in constituting construct systems - as Noaparast (2000) suggests - then the Psychology of Personal Constructs can be considered trapped. Not by the elegant reduction of language, but by the problems of the 'private language argument' outlined by Wittgenstein (1968).

\section{Ducking Past Words to the World Itself}

Further support for the inadequacy with which Kelly (1955) dealt with language and the implications of this inadequacy can be found within the work of Leman (1970). Beginning from the view that scientific activity is characteristically an operation with and occasionally on language, Leman (1970) contends that the most central issue confronting the scientist is the relationship between language and extralinguistic reality. Leman (1970) makes a distinction between what he terms "tangibles" (p. 134) or observations that exist and on the near side of language, and "intangibles" (p. 134) or hypotheses, theories, and thoughts that exist on the far side of language. He argues that it is language in the middle of these tangibles and intangibles that is customarily ignored by the scientific enterprise. ${ }^{35}$ For Leman (1970), the ignorance of this language in the middle is so widespread that "we would have to suppose that it really is possible (say) to observe without using language, to hypothesize without using language, to move back and forth between hypothesis and observation without moving through language" (p. 134). Instead Leman (1970) argues that there is an essential immixture of language in experience as well as language in thought. In fact, for Leman

\footnotetext{
${ }^{35}$ Seeking to highlight the importance of language in experience, Leman (1970) explores the notion of Terministic Screens as first proposed by Burke (1966). Burke (1966) was once shown photographs of astrological bodies that had been taken using narrow-pass colour filters (screens) that allow only light of one colour to reach the negative and thus not the full spectrum of colour possible. Drawing a comparison therefore between a Terministic Screen and one of Kelly's constructs, Leman (1970) suggests that when a person 'clicks' a Terministic Screen into the "apparatus between one's ears" ( $p$. 135), it is the screen itself that limits that which can be seen. In other word, the construct determines or rather limits that which can be seen. Burke (1966) foresaw a synergy between the filter screens and a series of words and phrases used by scientists as standing between the observed and the observer, preventing us from seeing all that is available to see. For a more complete discussion of Burke's notion of Terministic Screens, see Burke (1966). Language as symbolic action: Essays on life, literature and method.
} 
(1970), "this world, whether we like it or not, is effectively made of language; there is no least possibility of ducking past words to the world itself - the world itself is words (and word-like things)" (p. 148). In reaching toward a critique of Kelly's (1955) position, Leman (1970) therefore posits that "it is a mistake to talk about thought, or about the world outside thought," as Kelly does, "instead of talking about language and the world represented in language" (p.150). This is all the more a mistake from Leman's (1970) perspective given the direct and essential nature of language within the practice of clinical psychology.

It seems to me a disgracefully neglected fact, that clinical treatment of people without any discoverable organic disease is entirely a matter of talking (and of symbolic action of other kinds), that is to say, of use of verbal and nonverbal language (p. 150).

Although couched in a different way, Leman (1970) can be seen to critique Kelly's approach in the same way as Wittgenstein's (1968) critique of 'private language argument'does. Leman (1970) suggests that Kelly (1955) provides an 'operating manual' for going about the business of being human. However, given that the central features of this manual - the construct - are devoid of a capacity to come to language outside of the private words of the individual person, then it is difficult to apprehend how another person may see him or her self as embodied by this manual.

George Alexander Kelly spent a great deal of his time considering the problems of individuals: a person may, of course, face comparable personal questions, involving his whole private word model of the world outwith words he lives in - if you like, he has the problem of writing an operating manual for flesh ship Me, the lump of meat in which he finds himself (as language develops) self-consciously embarked as Captain and Navigator. (p. 150)

Leman (1970) goes on to argue that there is no conceivable means by which a person can go about writing such an 'operating manual' without recourse to a shared language, "the language of the larger manual" (p. 150). It is sufficiently clear for Leman (1970) "that there is no way in which we can duck through or past language (in the largest sense) to immediate experience of extralinguistic reality" (p. 151). Here Leman (1970) underpins further the arguments established by this thesis. In situating the construct beyond the reach of language, it is theoretically challenging to apprehend the way in which a person engages in the movement of abstraction that Kelly (1955) envisages. Stated another way, by not locating the being of the construct within the medium of language, we cannot begin to engage with the 
construct in the movement of ongoing abstraction that Kelly's (1955) position requires. ${ }^{36}$ Noting the theoretical problems inherent in this position, Leman (1970) cites the work of Otto Neurath.

We are like sailors obliged to rebuild our ship on the open sea, without ever being able to knock it down completely in dry dock and build it anew from the best of replacement parts. (p. 152)

Using the ship as an analogy for a person's greater system of constructs provides a useful vehicle for understanding the theoretical challenges of Kelly's position. In the absence of language as a medium for the construct, the movement or process of abstraction that ultimately underpins the process of reconstrual and the elaboration of our constructs, requires - to return to the analogy and in this instance the words of Quine (1960) - that we rebuild our construct system "plank by plank while staying afloat in it" (p. 3). Such a position is ultimately theoretically unsustainable.

\section{Language as a Filter for Constructs}

Offering a consideration of the ideological dimensions of the Psychology of Personal Constructs, Solas $(1992,1995)$ also makes a similar point. Through an engagement with Derridean deconstruction, Solas offers a possible extension to the social dimensions of construing by emphasising the points of intersection and difference between these two theoretical perspectives. ${ }^{37}$ For Solas (1995), interactions with other people operate to invalidate, confirm and elaborate constructions of the social world as well as providing grounds for similarity. In pursuing a similar direction to the argument levelled by this thesis, Solas (1995) makes reference to the work of Derrida (1981) as well as that of Leman (1970). Both Derrida and Leman agree on language being the condition of human thought and therefore the impossibility of theorising meaning that is anterior to its expression in words. On the basis of this argument, Solas (1995) stresses the need for Kelly (1955) to place a greater emphasis upon language. In fact, emphasising the ideological elements of Personal

\footnotetext{
${ }^{36}$ Proctor (2009) provides further fertile ground for this argument. For Proctor (2009), "constructs are not verbal...but a construct itself is an experienced discrimination" (p. 23). However, how is it that we can have an experience in the Kellyian sense, or make these discriminations, if indeed the construct cannot come to language in the movement of abstraction?

${ }^{37}$ More recently Strong and Shultz (2010) also provide a consideration of 'deconstructive' principles in the practice of counselling. These authors highlight, as does Solas (1995), the overlap between the Psychology of Personal Constructs and Derrida's deconstruction with specific regard for the social consensus that is implicit in understanding another person. Although Strong and Shultz (2010) offer a brief consideration of the importance of language in deconstructive accounts of reality, they do stop short of providing a thoroughgoing consideration of the theoretical implications of Kelly's (1955) inadequate account of language upon Personal Construct Psychology.
} 
Construct Psychology, Solas (1992) criticised Kelly's (1955) idea that constructs and the related tasks of meaning-making could in fact be non-linguistic.

It is apparent that constructs do not drift about the world like clouds in a summer sky, occasionally divulging themselves in a clap of thunder and a flash of light, but rather circulate in the world in specific forms of discourse, particular ways of writing, thinking and talking. (p. 382)

Despite Kelly's suggestion that the construct is beyond the reach of language, Solas's (1995) reading of Kelly (1955) suggests that, "even if, as Kelly maintained, verbal labels are proxies for constructs, language still acts as a filter through which construing has to pass" (p. 66). ${ }^{38}$ Here Solas (1995) confirms that in the absence of language as the medium of the construct, the process of abstraction that is necessitated by Kelly's Fundamental Postulate and elaborative corollaries is a theoretically untenable proposition to sustain. ${ }^{39}$

In line with these arguments, then, this thesis suggests that Kelly's failure to recognise that the medium of language is that within and through which we develop, nourish, and sustain our constructs, challenges the theoretical coherence of his position. This challenge can best be captured by way of a question: In the absence of language, how does the person that Kelly concerns himself with engage in the movement of abstraction that is requisite of his Fundamental Postulate and elaborative corollaries? In answer to this question; by situating the construct beyond the reach of language, Kelly (1955) locates the construct as a theoretical structure of which nothing can be said. If this is the case, then the words of Wittgenstein (1968) are telling: "A nothing would serve just as well as a something about which nothing can be said" (p. 102).

Although the foregoing has made it clear that the construct has its being beyond the reach of language, the question remains: 'Why should one be concerned with such matters as the parallels between language and the construct?' The short answer involves a concern for legitimacy on the part of those who subscribe to the theoretical tenets of Personal Construct Psychology. At the core of this legitimacy is a requirement for the construct itself to come to

\footnotetext{
${ }^{38}$ Although Solas (1995) makes the claim that verbal labels are proxies for the construct, it is clear from the previous discussion that Kelly (1955) was in fact adamant that the construct was beyond the reach of language, even at the level of proxy signifier.

${ }^{39}$ Solas's (1992) critique of the adequacy with which Kelly dealt with language also points towards further implications for Kelly's Sociality Corollary. At the centre of this challenge is a view that meaning is public and therefore is most essentially a matter of "interindividual intelligibility" (p. 382). That is, meaning is only made possible by the presence of a socially construed system of language. For Solas (1995) the social consensus that is implicit in such a position has its foundation in language, and represents a difficult position for Kelly to sustain given the way that he situated the construct outside of language.
} 
language. Therefore, if the construct is beyond the reach of language, then Personal Construct Psychology is open to the charge of being merely a relativistic position. On the other hand, if a theoretical connection with the domain of realism can be established, Personal Construct Psychology may avoid what Warren (1998) describes as "the charge" of being "merely subjective or merely a relativistic position" (p. 57). However, staving off this charge will require that the construct can be shown to have a link to language, a connection already shown to be theoretically incoherent. ${ }^{40}$ The following discussion will therefore explore the claim that Personal Construct Psychology can make to 'a realism'. ${ }^{41}$ Not only will this exploration outline further the implications of Kelly's inadequate dealings with language, it will operate to inform the theoretical development that is proposed by this thesis.

\section{Personal Construct Psychology and Realism}

A most lively debate within the domain of the Psychology of Personal Constructs involves its alignment with a series of 'isms' that are common in traditional philosophy. The most important of these for this thesis is a consideration of the claim made by the Psychology of Personal Constructs to a realism. ${ }^{42}$ In light of this, two of the key realist positions within the domain of Personal Construct Psychology - Noaparast's (1995) and Stevens' (1998) -

\footnotetext{
${ }^{40}$ Some authors have identified the Psychology of Personal Constructs as productively adopting a 'relativistic' position. Potter (1996, 1998), Erfran and Clarfield (1992) and more recently Raskin $(2001,2008)$ contend that a 'relativistic' position is not the dangerous or anything goes nihilism in the terms identified by others (Gillett, 1998; Held, 1995; Mackay, 2003; Parker, 1999). Instead, for Raskin, a relativistic position is an important and necessary element of a construct's evolution. As Raskin (2001) puts it, "even if I view myself as a realist/absolutist, I fleetingly become a relativist the instant I revise any constructs" (p. 13). Seeking to close down the relativism versus realism debate, and the subsequent conjecture about the relationship between subject and object, Chiari and Nuzzo (1996b, 2004) elaborate a Hermeneutic Constructivism that they believe overcomes this subject object dualism. Hermeneutic Constructivism will be elaborated at a later juncture in this thesis.

${ }^{41}$ It is interesting to note that for Noaparast and Khosravi (2006) Piaget took a vital step in reconciling the polarity of realism and constructivism. They argue that "Piaget is an important historical case for this exception as he talks about himself as a realist constructivist" (p.21).

${ }^{42}$ A testament to the lively nature of the debate that centres around a concern for the Psychology of Personal Constructs and a less common ism can be seen in the dialogue between Mackay (1994, 1997, 2003), Raskin and Neimeyer (2003) and McNamee (2003a). Mackay (2003), in line with the work of Held (1995a, 1995b, 1996, 1998, 2007), charges both constructivist and constructionist approaches of subscribing to anti-realism. At the core of his argument is a belief that positions such as Kelly's do not posit a reality independent of our constructions of it. Ultimately in such approaches there is no position to justify one take on reality over another (Held, 1995a, 1995b, 1998, 2007). However, Raskin and Neimeyer (2003) and McNamee (2003a) argue otherwise. Raskin and Neimeyer (2003) in defence of Kelly's (1955) position, suggest that Mackay (2003) has only a limited appreciation of Personal Construct Psychology and suggest that he is not forthcoming with the way that a person comes to know the supposedly fixed reality independently of his or her mental processes. McNamee (2003a, 2003b), in defence of social constructivism, suggests that the focus of the discipline upon discourse means realism is ultimately a socially mediated activity. For a more thorough consideration of this work see: Mackay (2003). 'Psychotherapy and the idea of meaning'; Raskin and Neimeyer (2003). 'Coherent constructivism: a response to Mackay'. McNamee (2003a). Bridging incommensurate discourses.
} 
will now be illuminated in order to provide a vehicle in order to highlight the theoretical implications of Kelly's (1955) problematic depiction of language.

\section{The Realist Version}

As has been seen, Kelly (1955) was explicit about many of his convictions, and the nature of the world that he envisaged was no exception.

We presume that the universe is really existing and that man is gradually coming to understand it. By taking this position we attempt to make clear from the outset that it is a real world we shall be talking about, not a world composed solely of the flitting shadows of people's thoughts. (p. 6)

The realist overtones within this statement are clear. However, later, Kelly (1955) made his rejection of a realist position equally as clear.

Since we insist that man can erect his own alternative approaches to reality, we are out of line with traditional realism, which insists that he is always the victim of his circumstances. (p. 17)

Despite this, Warren (1985) has argued that Kelly's rejection of realism upon these grounds was “too hasty" (p. 263). In fact Warren (1985) goes on to suggest that Kelly's position on realism represents a most extreme position that does not equate to more commonly accepted understandings of realism as detailing "a world of material things existing independently of our apprehension of it, but to which in apprehending we accord meaning" (p. 263). In terms of this more commonly held account of realism, Warren (1985) suggests that Personal Construct Psychology is quite consistent. It could therefore be argued that Warren's (1985) recognition of Kelly's overhasty rejection of traditional realism provided a pathway for others - for example Mair (1991), Neimeyer (1993) and Rowe (1993) - to consider Kelly's work in terms of a critical realism. Critical realism represents a position that arises from the perceived parallels between Kelly's recognition of an independent reality that we have access to only by way of our constructs, and Kant's idea that we can know the world only by way of what he termed the 'categories of understanding'. A brief consideration of these parallels is helpful for the further unpacking of the Kelly's claim to realism. ${ }^{43}$

\footnotetext{
${ }^{43}$ For a further discussion of the perceived parallels between Kelly and and some major philosophical writings, including Kant, see Proctor, H. (2011). 'The roots of Kellyian notions in philosophy: the categorical philosophers - Kant, Hegel and Peirce.'
} 
Kant argued that human beings are incapable of knowing the 'things-in-themselves' or noumena. Instead, he suggested that we come to know the world through the observable manifestations of those 'things' as phenomena. Kant argued that the mind imposes its own inherent structures - categories - upon the particulars of perception and that these categories in turn constrain our knowledge of the world. ${ }^{44}$ In line with this, Neimeyer (1993) suggested that when Personal Construct Psychology is viewed in terms of a critical realism it could be considered as being aligned with a postmodern rejection of positivist notions of realism.

Central to this position is an acceptance that a person's construct system cannot be thought of in terms of a 'match' with external reality, but rather as having critical points of contact with the external reality. These points of contact between our constructs and reality allow us to go on in the world - that is, these constructs are workable. As Raskin (2011) suggests, in an essentially pragmatic account such as this, the value of knowledge lies in its function, or utility, whereby knowledge is a tool people use to accomplish desired ends. Stevens (1998) also acknowledged the importance of a construct's workability for Kelly as emphasised in this following passage.

Kelly is more than a little slippery on this point about truth. He appears to want to make an each-way bet (or, to mix metaphors, he wants to have his cake and eat it too). Although truth is very important to Kelly as a philosophical assumption, in practice he adhered closely to Dewey's instrumentalism psychologically. According to Dewey, ideas and theories are tools or instruments that we use to operate on the world. He replaced the notion of truth by one of predictive usefulness. (p. 293)

Stevens (1998) argues that Kelly does not justify the realist assumptions within his theoretical position and instead simply relies on them in order to avoid a charge of thoroughgoing relativism. ${ }^{45}$ In order to 'rescue' Personal Construct Psychology from a tendency to

\footnotetext{
${ }^{44}$ For Mahoney (1988), the way in which Kant advocated for a 'top-down' epistemology that subsequently advanced a proactive consideration of human cognition, revolutionised philosophy and psychology.

${ }^{45}$ Warren $(1992,1998)$ also draws upon the work of Deutscher who considers the use of the verbs 'objecting' and 'subjecting' as suitable replacements for the notions of subjectivity and objectivity. Deutscher (1983) suggests that the notions of objectivity and subjectivity are distortions that originate from an attempt to escape our indissoluble involvement with and of the world, a world that is social by its very nature. It is worth noting here also that in this same paper Warren (1992) suggests, "Personal construct theory gives credibility and encouragement to the dialogic articulation of and elaboration of the individual's construct system" (p. 65). Drawing upon the work of Deutscher (1983), Barbu (1956) and Bernstein (1983) (who elaborated a dialogue between Habermas and Gadamer), Warren foresees an avenue to pursue a consideration of the problems that have surfaced more intensely in recent times with regard to notions of objectivity, subjectivity and relativism. During the unpacking of his essential arguments, Warren (1992) suggests that one does not "just dialogue", rather one always dialogues about something as "the object to which we turn our subjectivity" (p. 60). It follows then that for
} 
relativism, Stevens (1998) suggests that the addition of a 'minimum realism' to Kelly's theoretical position is required.

\section{Minimum Realism}

Stevens (1998) developed what he termed a 'minimum realism' that accepts that some aspects of reality are independent of a person's sense experience. In so doing he proposes that it is indeed a real world that we construe, a position that he suggests can overcome a simple ontological constructivism. ${ }^{46}$ Here Stevens (1998) recalls the work of Rescher (1987) - cited also by Noaparast (1995) - who argues that the world has an "error tolerance" (p. 75) that provides for the possibilities of competing hypotheses and constructions of it. Stevens (1998) argues that "although a theory may be adequate (predictive or viable), its assumptions may demonstrably not be true" (p. 287), and goes on to suggest that "our personal constructions may be currently viable, but this does not necessarily imply they have a hold on truth in any final or complete sense" (p. 287). As a result, Stevens (1998) argues that the constraining power of reality is a theoretical predicate of the Psychology of Personal Constructs and as such it must assume a minimum realism if it is to avoid philosophical relativism.

Stevens (1998) recognises that an adequate theory of human understanding must be cognisant of non-reflexive first-order knowing and reflexive second-order knowing. ${ }^{47}$ Stevens (1998) goes on to describes first-order knowing as a knowing "unaware of its own processes, a knowing that, presumably we have in common with the animal kingdom - a knowing that is, nonetheless, indispensable for the emergence of human understanding" (p.288). He goes on to outline second-order knowing in the following way, "once emergent, all human knowing is potentially permeated by second-order knowing. This is a reflexive understanding, constructed within and through language and culture" (p. 288). In order to apply these levels of knowing to Personal Construct Psychology, Stevens (1998) calls on the work of Searle (1995) and his idea of a background. This background provides an assumed

Warren (1992), when one dialogues it is indeed possible that he or she dialogues about their constructions and bring their constructions to language. This manoeuvre however is clearly out of step with the way in which Kelly (1955) conceptualised the construct as being outside the reach of language. To argue otherwise would surely be to have moved beyond the boundaries of the Fundamental postulate of The Psychology of Personal Constructs.

${ }^{46} \mathrm{~A}$ consideration of ontological constructivism is undertaken in the examination of Noaparast's Sophisticated Realism that follows. Ontological constructivism is a position whereby a person's constructs are considered to have value if they are workable over and above being real.

${ }^{47}$ Here Stevens (1998) can be seen to make some clear synergies between his work and that of Margolis' (1978) notion of natural entities and culturally emergent entities. Moreover, Stevens (1998) draws on what Huder (1995) called one's faculties of sensibility and understanding and what Harre (1989) referred to as two realities (physiological and social) in establishing the ideas of first and second-order knowing. 
external realism that operates as a condition of intelligibility for all that is said and thought. Therefore, some constructs are needed in order to maintain a contact with the world, while the others are being 'worked out' against the background of reality. Worded another way, this background is contingent upon some constructs having what Stevens (1998) describes as an ontological counterpart. ${ }^{48}$ This process of using some constructs to establish a reality while abstracting other constructs is suggested by Stevens (1998) to be the task of first and second order knowing. The following passage highlights the requirement of this ontological connectedness for Stevens (1998).

If it is argued that this 'surveying' or 'abstracting' is meant to be purely projective, then it is hard to imagine how our subsequent behaviour (understood in the Kellyan sense as an experiment made on the basis of that abstracting process) can ever test the original construct. For all we could ever experience in the world would be our system once again projected onto a neutral universe. But this is not our experience of, nor Kelly's position regarding reality. (p. 299)

To overcome this conundrum, Stevens (1998) recalls the work of Husain (1983) and his idea that the world has a voice in the dialectic conversation experienced as living. This thesis suggests, however, that these theoretical manoeuvres - not unlike those of Noaparast (1995) represent a theoretical discordance between Steven (1998) minimum realism and Kelly's (1955) position. Stevens (1998) - in line with Kelly's (1955) position - is clear that it is a person's constructions that provide access to the world that he or she comes to know. This, for Stevens (1998), takes the form of a first-order knowing, which he suggests is then able to come to language as a second-order knowing. However, if we are to remain consistent with the way that Kelly (1955) situates the construct beyond the reach of language, then this second-order knowing is theoretically inconsistent. That is, if a person's constructions which cannot be anything other than a first-order knowing - are to emerge in and through a second-order knowing as language, then clearly Stevens (1998) and Kelly (1955) are at odds. In fact, this theoretical disconnect is such that Stevens (1998) could be considered to have moved beyond the domain of Kelly's (1955) Fundamental Postulate to an entirely different position all together.

The implications of Kelly (1955) situating the construct beyond the reach of language are again evident. If the minimum realism Stevens (1998) propounded is to be coherent, then the person Kelly (1955) conceived of would need to engage - in a dialogue - with this

\footnotetext{
${ }^{48}$ This is a position that Steven (1998) borrows from the work of Husain (1983).
} 
background reality in order to abstract further his or her constructs. Instead, the person that Kelly (1955) conceived of can only ever be a mute participant in this dialogue with the world, given the separation of the construct from language. A closer examination of Noaparast's (1995) conception of a sophisticated realism - a position that Stevens (1998) was openly indebted to - will further emphasise the implications of Kelly (1955) situating the construct beyond language and more specifically with regard to the claim to realism that the Psychology of Personal Constructs can make.

\section{Sophisticated Realism}

Noaparast (1995) argues that work such as that by Warren (1991) - who suggested that Kelly's work could be considered in terms of an epistemological realism given that it is concerned with the validity of ideas - has left the Psychology of Personal Constructs open to the challenge of instrumentalism. ${ }^{49}$ Indeed the task that Noaparast (1995) set for himself was to rescue the Psychology of Personal Constructs from the challenge of instrumentalism. It is with regard to this aim that Noaparast (1995) attempts to situate the Psychology of Personal Constructs with a sophisticated realism. At the core of sophisticated realism, however, is the requirement for a person to bring to language his or her constructions, or what Noaparast (1995) terms a person's ‘best theory'. Again, however, it becomes clear that Kelly's (1955) conviction that the construct is located beyond language makes the carriage of Noaparast's (1995) sophisticated realism a difficult proposition to sustain and again raises doubt about the overall theoretical tenability of Personal Construct Psychology. A brief sketch of Noaparast (1995) claim is facilitative.

Noaparast (1995) suggests that despite the efforts of other authors ${ }^{50}$ within the field of Personal Construct Psychology, the task remains to elucidate an understanding of the epistemological characteristics of Kelly's constructivism. For Noaparast (1995), instrumentalism represents an epistemology whereby "a workable theory is true because it works" (p. 39). However, in seeking ultimately to overcome this view, Noaparast (1995) suggests a contrary argument.

\footnotetext{
${ }^{49}$ Noaparast (1995) suggests that what Warren (1991) had actually disclosed was an ontological realism. Tarski (1944) however, suggests that an appeal to an ontological realism cannot account for an epistemological realism. That is, an ontological realism does not account for a belief in a real world or the way in which our constructions of it are indeed real.

${ }^{50}$ Noaparast (1995) recalls the work of Mancini and Semerari (1988) and Rowe (1993) who identified some similarities between the theoretical positions of Kelly and Popper. However, in an effort to ensure the contemporaneous nature of Kelly's work, Neimeyer (1993) suggested a postmodern rejection of the positivist view of science was supported by Kelly's (1955) position, while Tschudi (1983), supporting a similar position, suggested that fact theory distinctions are not sustainable and thereby challenged the notion of objective verifiability.
} 
The workability of a theory does not constitute its truth on the ground that there could be reason, other than its being true, for a theory to work and more specifically in relation to one's system of personal constructs while an incoherent system cannot be true, a coherent system is not necessarily true. (pp. 47, 48)

Noaparast (1995) suggests, that in selecting one construct from two workable constructs, the determinant factor is ultimately the specific construct's coherence with existing constructions. That is, we choose one construct over another because it sits comfortably within our greater system of existing constructs. However, Noaparast (1995) goes on to highlight that underpinning this coherence is first and foremost the workability of the construct with which coherence is sought. In other words, the constructs that a person already has in his or her construction system have become a part of that system on the basis of their workability as constructs. Thus, any subsequent construct coherence represents a coherence ultimately with the workability of that construct that we already have. For Noaparast (1995) then, a construct coherence that is ultimately reliant upon workability is not sufficient to overcome instrumentalism and is therefore insufficient to rescue the Psychology of Personal Constructs from a challenge of instrumentalism. ${ }^{51}$

In seeking to overcome this identified instrumentalism, Noaparast (1995) proposes a sophisticated realism that has five general tenets. ${ }^{52}$ These tenets he outlined as follows:

(a) there is a real world independent of our theorizing, (b) our theories can account for this reality, (c) fact-theory distinctions are not acceptable, (d) any simple correspondence between our theories and reality is rejected, and (e) our true sentences correspond to the reality of the world captured by our best theories. (p. 50)

With regard to these tenets, ${ }^{53}$ it is with the assertion that 'our true sentences correspond to the reality of the world captured by our best theories', ${ }^{54}$ that this thesis suggests is problematic for

\footnotetext{
${ }^{51}$ Noaparast (1995) argued that it is the profound influence of early pragmatists - most notably Dewey - and pragmatism's own instrumentalism, that leads Kelly (1955) toward instrumentalism.

${ }^{52}$ The foundation of these tenets is not unfamiliar to the work of Quine (1960) who in turn absorbs some of the theoretical tenets of Tarski (1944) and from which Noaparast draws inspiration.

${ }^{53}$ The first three of these tenets are clearly embodied within the work of Kelly (1955) as outlined here:

(i) Kelly is clear that it is real world that is being dealt with; (ii) the personal construct system is clearly the theoretical position able to deal with the world; and (iii) Kelly establishes that the construct system (theory) is the means by which the world (fact) comes to meaning and as such overcomes a distinction between fact and theory. Stating that "any simple correspondence between our theories and reality is rejected", Noaparast (1995) argues through his fourth tenet that a construct's coherence within the wider construction system beyond a naïve or simple correspondence with reality itself operates to
} 
establishing a sophisticated realism. A passage from the work of Stevens (1998), describing Noaparast's (1995) sophisticated realism, facilitates an appreciation of these theoretical concerns.

This theory of truth is 'intratheoretic' and not objectivist: that correspondence is between statements and a theory's grasp on reality (not directly between statements and reality itself). It is not relativistic, however, because the theory is always subject to rejection if not supported by evidence. (p. 295)

There is more than an analogy here between what Noaparast (1995) is suggesting as 'one's best theory' and Kelly's (1955) system of constructs. In fact, Stevens (1998) goes on to suggest that, "taken as a whole, the construct system operates much like a personal theory of the world" (p. 295). In bringing further clarity to the relationship between true sentences and best theory, the following passage from Noaparast (1995) is useful.

We can use the chosen theory to maintain what exists in the world and to what our true sentences correspond. In other words, our best theory is a metalanguage that has a grasp of reality. In the context of this metalanguage, we can talk of correspondence of our sentences to this grasp. (p. 53)

It is important to establish that Noaparast (1995) is not suggesting a simple or naïve correspondence between facts and sentences. Rather, he is suggesting a sophisticated correspondence between sentences and our best theories - our construct system - or our grasp of reality. In conceptualising truth in terms of correspondence and not instrumentalism, and on the basis of what Noaparast (1995) termed evidence, a person's best theories or constructions are deemed valid based on their grasp of reality, and not simply their correspondence with other already existing and therefore workable constructs.

overcome this challenge. However, this poses a theoretical conundrum with regard to the formation of a person's initial construct. In the absence of a greater construct system with which it could cohere as would be the case in the early development of a construct system - this is difficult to apprehend. Seeking to have the argument both ways, Noaparast (1995) suggests that we may need to defer to the workability of a construct in this instant. Here, and unavoidably, Noaparast (1955) makes a return to the ontological realism he fought to overcome. Noaparast (1995) suggests that the conceptualisation of a metalanguage of the construct system provides an intermediary for determining the degree of fit between the construct and reality and he believes it may operate to overcome some of these theoretical issues. However, a critique of the final tenet - a critique that concerns the bringing of our best theory to language - will suggest otherwise.

${ }^{54}$ The original passage within the work of Quine (1960) from which this statement arises is as follows: "It is rather when we turn back into the midst of an actually present theory, at least hypothetically accepted, that we can and do speak sensibly of this and that sentences as true. When it makes sense to apply 'true' is to a sentence couched in the terms of a given theory and seen from within the theory complete with its posited reality" (p. 24). 
The synergies between what Noaparast (1995) suggested as one's best theory and what Kelly (1995) termed construct system are palpable. Recalling Kelly's (1955) introduction to the construct furthers these synergies.

Man looks at the world through transparent templates which he creates and then attempts to fit over the realities of which the world is composed...without such patterns...man is unable to make any sense out of it. (p. 9)

Thus, the anticipatory nature of the construct and the greater construct system ultimately provides the person that Kelly (1955) envisaged with his or her grasp on reality, or best theory. However, given that Kelly (1955) locates the construct beyond the reach of language, the way in which a person establishes a correspondence between one's 'best theory' or construct system and his or her true sentences, makes 'sophisticated realism' a theoretically challenging position to sustain. Indeed, as has already been highlighted in this chapter, Noaparast's (2000) paper titled 'Constructs and Words', acknowledged the discordant nature of any relationship between language and the construct. In so doing, Noaparast (2000) problematises the compatibility between the Psychology of Personal Constructs and his own sophisticated realism.

What is doubtful is the reductive claim that all constructs have linguistic nature....In summary, the fundamental position taken by Kelly to the effect that constructs are not necessarily sentential is a touchstone in personal construct psychology, which prevents this theory from being sunk in the long and multifaceted tradition of reductionism. (p. 69)

In light of this acknowledgement from Noaparast (2000) and the preceding argument, if a person's constructs cannot come to language - as Kelly (1955) is most adamant - then it follows that this person's true sentences cannot correspond to his or her construct system or best theory. Thus, Personal Construct Psychology is incompatible with a sophisticated realism. To attempt to lay claim to the contrary would be to do one of two things: firstly, to move beyond Kelly's (1955) elaboration of his Fundamental Postulate, a position Kelly strongly resisted, or secondly, to assume a naïve correspondence between a person's true sentences and his or her construct(s). ${ }^{55}$ This latter position is certainly incompatible with

\footnotetext{
${ }^{55}$ In elaborating the fundamental postulate, Kelly (1955) stipulates that "one should bear in mind that the moment we do question the truth of a statement proposed as a postulate, that statement is no longer a postulate in our subsequent discourse...If we bring the statement into dispute, as well we may in some instances, we must recognise we are then arguing from other postulates either explicitly stated or, more likely, implicitly believed. Thus, in scientific reasoning nothing antecedes the postulate, as long
} 
another of the tenets that Noaparast (1995) proposed for his 'sophisticated realism' that rejects any simple correspondence. ${ }^{56}$

\section{No Realism Without Language}

The implications of Kelly's (1955) situating the construct beyond the reach of language have been shown by this thesis to be significant to the theoretical sensibility of the Psychology of Personal Constructs. Locating the being of the very structure with which the world comes to understanding outside of language, raises doubts about the degree to which such a theoretical position is able to provide a sustainable, reflexive and legitimate 'inner outlook' of the individual person. The broader implications of these arguments are significant to this thesis.

Embodying the principles of the idiographic perspective about which this thesis holds most hope, the Psychology of Personal Constructs was to be the underpinning theoretical position from which to begin the development of a new theoretical approach for qualitative research. However, most certainly the touchstone of any theoretical foundation for qualitative research, is the theorising of an inextricability of language from the structures of being human. Given that the construct as the most fundamental element of being for Kelly is beyond the reach of language, this thesis shares the view propounded by Wittgenstein (1953/1968) of such theoretical manoeuvres, "what is hidden... is of no interest to us...a nothing would serve just as well as a something about which nothing can be said" (pp. 50, 102). Thus, the theoretical foundations of the Psychology of Personal Constructs is considered incommensurate with the task of providing a complete foundation for developing an approach for catching a glimpse of the 'inner outlook' of the individual person going about the business of being human for the purposes of qualitative research.

It must be said, nonetheless, that this incommensurability is one of degrees. In fact, several of the theoretical and philosophical principles embodied by the Psychology of Personal Constructs will ultimately be 'read between many of the lines' of the final theoretical position that is developed by this thesis. In seeking to move forward with the endeavour of developing this new theoretical position for qualitative research, this thesis suggests that we go on 'as if' language is indeed the missing medium within the Psychology of Personal Constructs. This notion of language as a medium is a position that is most

as it is a postulate, and the truth of a statement is never questioned as long as that statement is in use as a postulate" (p. 47).

${ }^{56}$ This manoeuvre would be inconsistent with tenet (d) of Noaparast's (1995) discussion of the general tenets of 'sophisticated realism'. This tenet suggests that any simple correspondence between our theories and reality is rejected. 
comfortably elaborated by hermeneutics. An examination of the theoretical elements of hermeneutics will provide a foundation from which to re-envision several of the theoretical principles of Personal Construct Psychology that have been problematised here. It is anticipated that this re-envisioning of Kelly's work may provide a theoretical avenue that proves valuable in catching a glimpse of the 'inner outlook' of the individual person for qualitative research.

\section{In the Direction of Language}

In suggesting that we go on 'as if' language is the missing medium, it is important to first distinguish what is meant by the term medium. This thesis takes the view - in line with the expressive account of language - that language is that within and through which the world comes to be understood. It is therefore the essential medium for going about the business of being human. In approaches where language is a medium the person does not simply have an experience of the world and then name it as a function of a reflection. Rather, each person brings those aspects towards which one directs his or her understanding processes to language and only in so doing experiences the world. Thus, where language is the medium of being human, the way in which a thing comes to be understood - including our Self-is not different from the way in which that thing comes to language. However, prior to going on in the direction of hermeneutics 'as if' language is the medium of being human, it is important to acknowledge that several of the links between Personal Construct Psychology and hermeneutics have been noted previously. A sketch of these links is provided below.

\section{Hermeneutics and The Psychology of Personal Constructs}

The view that "personal construct psychology can be seen as a member of the phenomenological family" (Butt, 2003, p. 386) is supported amongst proponents of Kelly's (1955) work (cf. Butt, 1998; 1999, 2003, 2004, 2005; Butt \& Langdridge, 2003; Chiari \& Nuzzo, 1988, 1996a, 1996b, 2000, 2004a, 2004b, 2010; Warren, 1985, 1989, 1990, 1991, 1992, 1998, 2000). Extending the compatibilities beyond phenomenology, Warren (1998) provides a case for locating Personal Construct Psychology within a hermeneutic tradition and his work can be considered to lend support to the pursuit of a hermeneutic elaboration. ${ }^{57}$

\footnotetext{
${ }^{57}$ For further support of the hermeneutic elaboration of constructivist approaches - particularly critical constructivisms - can be seen in the work of Goncalves $(1994,1995)$. At the foundation of his work is a vision that a more hermeutic approach will support a view where humans are seen "neither as computers nor scientists, but as artists simultaneously portraying the role of actors and directors" (1995, p. 222). It is interesting to note that Epting (1988), according to Chiari and Nuzzo (2000), "has often declared his hermeneutic interpretation of personal construct psychotherapy", as outlined in Epting's (1988) 'Experiential Personal Construct Psychotherapy', arguably the first hint of a
} 
So much so, that Warren (2000) suggests that "there appears, then to be a close fit between the perspectives offered by hermeneutics and personal construct psychology and this opens up a richer understanding of our functioning" (p. 88) ${ }^{58}$ Although Warren remains one of the most highly regarded thinkers in the areas concerning the "philosophical dimensions of Personal Construct Psychology', his theorising of the connections between hermeneutic thought and Personal Construct Psychology does not extend beyond recognition of the productive possibilities of such a nexus, to a fleshed-out theoretical position itself. ${ }^{59}$ Nevertheless Warren's (1998) claim that “an understanding of personal construct psychology as a hermeneutic constructivism appears as defensible as an understanding of it as an epistemological constructivism" (p. 169), is a position now shared by several other scholars in the area of Personal Construct Psychology (cf. Butt, 2004b; Domenici, 2008; Epting, 1988; Goncalves, 1994, 1995; Raskin, 2002). More recently, Domenici (2008) suggested that Kelly's theory could be considered consistent with the hermeneutic perspective as conceptualised through the work of Chiari and Nuzzo (1996a, 1996b, 2010). Although this thesis will suggest that their approach is theoretically inconsistent with Kelly's (1955) position, Chiari and Nuzzo do provide the most sustained consideration of Kelly's theory as being situated within a hermeneutic domain. An exploratory outline of the work of Chiari and Nuzzo is thus clearly justified given the direction to be taken by this thesis.

\section{The Hermeneutic Constructivism of Chiari and Nuzzo}

Chiari and Nuzzo (1996b) were concerned that the general definition of constructivism was given to approaches that have at their core a proactive conceptualisation of the person. At the centre of their dissatisfaction is a belief that the terminology is inadequate for differentiating between an increasing number of approaches that share a proactive view of the human subject. With an increase in the approaches that are supposedly

hermeneutic consideration of Personal Construct Psychology. For a complete review of this work see: Epting (1988). 'Journeying in the personal constructs of children.'

${ }^{58}$ Warren $(1998,2000,2004)$ draws upon the work of Frank (1989) who provides a critique of the work of Derrida, Deleuze, Foucault and Gadamer on the basis of what he considered to be an insurmountable neo-structuralism. This argument draws Warren $(1998,2000,2004)$ towards the hermeneutics of Fredrick Schleiermacher who introduced to hermeneutic thought the notion of a psychological element. From Schleiermacher's position one had to look not only at the grammatical structures but also the psychological aspects in order to gain a complete an understanding as possible of the individual person (Warren, 2000). Schleiermacher developed the process of psychological 'divination' through which one can be considered to determine the viewpoint of another individual. For Warren (2000), such a conception provided a useful means of conceptualising the social aspects of meaning making, without a loss of the individual uniqueness. Despite this, a critique of Schleiermacher's 'psychologism' has been sustained by Gadamer and will be touched on during the following chapter. A more thorough exploration of the critique outlined by Gadamer can be seen in: Gadamer (1960/2003). Truth and Method (pp. 184-197).

${ }^{59}$ It must be said at this stage that going beyond such recognition was never a goal that Warren established for himself by way of his published work. 
embodied by the umbrella of constructivism has come a subsequent increase in the permeability of the term itself. Chiari and Nuzzo (1996b) believe this permeability is responsible for "a progressive dilution of its meaning" (p. 164). Therefore, in order to facilitate the differentiation of different approaches under the umbrella of constructivism, Chiari and Nuzzo (1996a, 1996b) identified what they believed to be the most significant point of discrimination. ${ }^{60}$ For these authors, at the centre of this discrimination is the way that different constructivist approaches deal with assumptions concerning the knowledge/reality relation. On this, Chiari and Nuzzo (2010) identify the difficulty for theorists abandoning the realist view without subscribing to its traditional opposite, namely, the idealist view.

We are inclined to swing between the idea of a reality demanding to be known as it is (exposing us to the danger of being wrong), and the idea that we can invent reality at our whim (and therefore that everything goes well). Science, obviously, embraces the first view and strives to discover what reality definitely is; looking with suspicion at the attempts to question such a premise. (p. 49)

These authors therefore apprehend constructivism as an effort to conceptually bridge the realist and idealist approaches to knowledge. They go on to suggest that approaches that grapple with bridging these idealist - realist conceptions are worthy of the title "psychological constructivism'.

\footnotetext{
${ }^{60}$ Not considered here in the distinctions between various constructivist approaches are those of Radical Constructivism or the better-known Social Constructionism. Despite this, Raskin (2008) believes the term constructivism relates to both personal construct psychology and radical constructivism, but not social constructionism. He goes on to suggest that radical and personal construct positions share a view of meaning that is more a process of the individual than a social process (Raskin, 2002, 2006). Radical constructivism envisages the mental meaning making activities of the person as a closed system that only he or she is in touch with (Maturana and Varela, 1992; von Glasersfeld, 1984, 1995). Thus, those within the realm of radical constructivism situate knowing as a private and individualistic undertaking. Despite the subscription to the possibility of a private language that is central to this view, the radical constructivism of Maturana and Varela (1992) locates language as being central to the activity of meaning making. However, the solipsism that follows from such a position makes radical constructivism a less suitable theoretical position for underpinning the theoretical elaboration for the purpose of this thesis. On the other hand, social constructionism locates relationships, rather than individuals, as the central feature of an approach for investigation. In this position, meaning making is a collaborative enterprise that cannot be traced to any particular individual. The work of Berger and Luckman (1966) The social Construction of Reality, and Gergen $(1985,1994)$, represent significant touchstones within the discipline of social constructionism. Gergen $(1985,1994)$ is openly critical of the 'cognitive' nature of Personal Construct Psychology and suggests that Kelly's position reduces the person to his or her individual experiences. However, despite the centrality of language in the Social Constructionist position, it is the rejection of the potentialities of the construing individual person by social constructionists (McNamee, 2003a) that is incongruent with the idiographic aspirations of this thesis and arguably human being more generally. For a comprehensive overview of Radical Constructivism and Social Constructionism and its compatibility or otherwise with Personal Construct Psychology, see the article by: Raskin (2002). 'Constructivism in psychology: Personal construct psychology, radical constructivism, and social constructionism.’
} 
Under the 'psychological constructivism' label, Chiari and Nuzzo (1996a, 1996b) distinguish an 'epistemological constructivism' ${ }^{61}$ and a 'hermeneutic constructivism'. For these authors, epistemological constructivism specifies an ordering and organisation of the world on the basis of our experience, while hermeneutic constructivism is where "operations of distinction in language constitute the generation and validation of all reality" (p. 178). ${ }^{62}$ Thus for Chiari and Nuzzo (2010) hermeneutic constructivism operates to overcome the subject/object dichotomy by acknowledging a "mutual specification between knower and known" (p. 51), or what these thinkers describe as a "complementarity" (p. 53). ${ }^{63}$ For Chiari and Nuzzo, this complementarity is embodied by the notion of 'autopoesis' ${ }^{64}$ Autopoesis was first outlined by the biologists Maturana and Varela (1972/1980), who define this concept in the following way.

An autopoetic machine is a machine organized (defined as a unity) as a network of processes of production (transformation and destruction) of components which: (i) through their interactions and transformations continuously regenerate and realize the network of processes (relations) that produced the; and (ii) constitute it (the machine) as a concrete unity in space in which they (the components) exist by specifying the topological domain of its realization as such a network. (pp. 36/78)

Chiari and Nuzzo (1996a, 1996b, 2006, 2010) find several productive links between the work of Maturana and Varela (1972/1980) and Personal Construct Psychology when considered in terms of the idea of an autonomous self-organising system. ${ }^{65}$ When taken at face value, these similarities are palpable. ${ }^{6}$ However we must also recognise, in light of the argument outlined

\footnotetext{
${ }^{61}$ Raskin (2002) suggests that Chiari and Nuzzo's epistemological constructivism is open to those same challenges outlined previously of ontological realism.

${ }^{62}$ Chiari and Nuzzo (2010) emphasise the subject object dualism that they believe to be inherent within Kelly's (1955) work. To underpin this view they refer the following passage from Kelly (1955): "Life, to our way of thinking is more than mere change. It involves an interesting relationship between parts of our universe wherein one part, the living creature, is able to bring himself around to represent another part, his environment" (p. 8).

${ }^{63}$ For a greater consideration of this notion of complementarity see Chiari \& Nuzzo (2006) 'Exploring the sphere of between: The adoption of a framework of complementarity and its implications for a constructivist psychotherapy.'

${ }^{64}$ Perhaps the most widely regarded and thorough critique of 'autopoiesis' can be found within the work of Fleischaker (1992) 'Questions concerning the ontology of Autopoiesis and the limits of its utility'; Swenson (1992) 'Autocatakinetics, Yes-Autopoiesis, No: Steps to a unified theory of evolutionary ordering'; Kenny and Gardner (1988) 'The constructions of self organizing systems'. ${ }^{65}$ For Chiari and Nuzzo the structural determinism that is embodied by the system that Maturana and Varela (1972/1980) proposed, avoids many of the subject object challenges raised by viewing Personal Construct Psychology in terms of an epistemological constructivism.

${ }^{66}$ These surface similarities between the work of Kelly and Maturana are also identified by Feixas (1995) who argues that these authors "share more assumptions than not" (p.311). However, it must be said that he does go on to emphasise an ontological divergence between the work of these thinkers.
} 
previously, that the suggestion that Personal Construct Psychology and the notion of autopoiesis are theoretically compatible is open to contention. That is, in suggesting that Personal Construct Psychology sits comfortably with a theoretical position where a person can make abstractions through language in order to validate reality, Chiari and Nuzzo have indeed moved beyond the domain of Kelly's Fundamental Postulate and his broader theoretical position per se. ${ }^{67}$ Although Chiari and Nuzzo continue to seek to emphasise the parallels between the tenets of hermeneutics and Personal Construct Psychology, the following discussion will emphasise the incompatibility of this supposed relationship.

Chiari and Nuzzo (1996b) suggest that hermeneutic constructivism considers knowledge and truth in the following way.

An interpretation historically founded rather than timeless, contextually verifiable rather than universally valid, and linguistically generated and socially negotiated rather than cognitively and individually produced. (p.

However, in an article titled 'Steering Personal Construct Theory Toward Hermeneutic Constructivism', Chiari and Nuzzo's (2004) take the inherently hermeneutic tenets of historicity, embodied subjectivity and language, in order to highlight what they describe as their "tentative interpretation of PCT (Personal Construct Theory) as a hermeneutic constructivist theory" (p. 53). These authors go on to explore each of these tenets in terms of the work of Gadamer, Merleau-Ponty and Heidegger respectively. ${ }^{68}$ Here, a considered sketch of just two of these tenets - historicity and language - will underscore the tenuous nature of the relationship between hermeneutics, autopoiesis and the deeper aspects of Kelly's work.

\footnotetext{
${ }^{67}$ It is not the specific brief of this thesis to critique Chiari and Nuzzo's adoption of the tenets of 'autopoesis. Despite this, the failure of the 'autopoiesis' of Maturana and Varela (1980) to situate the essential structures of the self-organising system within the medium of language ultimately leads to the same argument levelled against Kelly's work and in fact operates to perpetuate a different dualism. In other words, the perturbations and recursion that Maturana and Varela (1980) elaborate as the fundamental aspect of the self-organising system are separate from the structures of the self-organising system. Thus, there is no theoretically coherent means by which these structures can be engaged in the movement of recursion or ongoing perturbation. Despite Maturana and Varela's (1972/1980) appeal to language and particularly to the notion of 'languaging' - as the activity of language use - the relationship between the structures of the autonomous organising human system and the medium of language is inadequately developed. This leaves Maturana and Varela's (1980) 'autopoietic' system susceptible to the theoretical slippage into the very 'dualism' that Chiari and Nuzzo work to overcome. This dualism is not so much a subject object dualism, rather a subject 'organising system' dualism.

${ }^{68}$ Although Chiari and Nuzzo (2004) propose the consideration of the Psychology of Personal Constructs against the backdrop of these tenets, the consistency of these tenets with the work of Kelly is not made more evident by the elaboration that these authors offer. Instead, Chiari and Nuzzo (2004) appeal more to the compatibility of these tenets with the work of Maturana and Varela.
} 
Chiari and Nuzzo (2004) take Gadamer's notion of historicity and, in linking it to the notion of recursion proposed by Maturana and Varela, propose that:

Historicity refers to the unavoidability to found any new interpretation on the constrained possibilities offered by the current structure of the knower, historicity can be viewed in terms of recursiveness. (p. 54)

Although Gadamer would agree with the idea that historicity is unavoidable, the choice of the word 'constraining' sits less comfortably with Gadamer's work. For Gadamer, as will be discussed in the next chapter, historicity provides the possibility of a person having understanding at all. That is, historicity provides the beginning point for further interpretation, as an essential and yet always only an initial position from which the infinite potentialities of understanding are possible. Taken in this way, historicity is the direct antithesis of 'constraining'. Moreover, the links between Kelly's work and the notion of historicity are all the more tenuous given his outright rejection of its importance. In fact Kelly (1955) makes clear that he considers the Psychology of Personal Constructs to be an 'ahistorical approach'.

The perceptual theories in psychology are frequently said to espouse the ahistorical approach to the understanding of behavior. This approach is from the viewpoint that one's activity at a given moment is determined primarily by his outlook at that moment. What has actually happened in the past can influence behavior only through the perceptions which are operating at the present instant. Personal-construct theory takes a somewhat similar stand. (p. 174)

With regard to the second hermeneutic tenet - language - Chiari and Nuzzo (2004) contend that it is crucially "important in the construction of realities and selves" (p. 57). However, the links between Personal Construct Psychology and their hermeneutic constructivist position become all the more ambiguous when their own statement about the importance of language is juxtaposed with their subsequent claim that Kelly (1955) has dealt inadequately with language. Moreover, the way in which Kelly (1955) situated the construct beyond the reach of language makes the task of establishing Personal Construct Psychology as a hermeneutic constructivist position theoretically challenging. In other words, the separation of the construct from language means that this alignment of Personal Construct Psychology with a hermeneutic constructivism or a hermeneutic position per se is questionable to say the least. 
These dubious parallels between Personal Construct Psychology and hermeneutics therefore fail to overcome the subject/object dualism that initially propelled Chiari and Nuzzo (1996a, 1996b). If the construct is outside of language - as Kelly (1955) makes clear - then the possibility of establishing what Chiari and Nuzzo (2010) describe as a "mutual specification between knower and known" (p. 51) is difficult to apprehend. Instead, these authors underscore the unbridgeable dualism that has been underscored by this thesis; that of the construct system and language.

\section{Chapter Summary}

This chapter has suggested that the way in which Kelly (1955) depicts language leaves the Psychology of Personal Constructs open to challenge in two ways. Firstly, the way that Kelly (1955) clearly situates the construct beyond the reach of language challenges the theoretical tenability of his position. Secondly, any claim that a construct can come to language - albeit inconsistent with Kelly's (1955) theorising - requires the subscription to a designative account of language. Here, words are assumed to simply correspond to a construct, and suggests Kelly's (1955) adherence to the possibility of a private language. Both of these challenges are informed by a consideration of the philosophical movements that underpin the Linguistic Turn. At the core of this view is an expressive account of language that suggests that language is itself disclosive of the world and is inextricable from thought. In fact, from this position the possibility of thought without language and the idea that a person can merely bypass language and go straight to thought itself, or in this case a person's constructs, is theoretically untenable. Wittgenstein (1968) culminates his 'private language argument', or the idea that an individual person can engage in a process of abstraction and yet be unable to articulate these abstractions to another person, by stating that "a nothing would serve just as well as a something about which nothing can be said" (p. 102). This chapter has emphasised that for Kelly (1955) that thing of which nothing can be said is in fact our constructs.

A consideration of the work of other scholars - Leman (1970), Noaparast (2000), and Solas $(1992,1995)$ - provided further support for the position established by this thesis. Collectively their work underscores the challenge levelled at the theoretical tenability of Kelly's position on the basis that he needed to take language more seriously. The implications of the way Kelly inadequately dealt with language upon the claim that Personal Construct Psychology can make to a realism were also considered. In order to demonstrate that the construct is founded within reality, and in so doing overcome a charge of simple relativism, scholars of Personal Construct Psychology defer most commonly to two common realist positions. However, the theoretical cogency of these positions is also open to 
challenge on the basis that Kelly clearly situates the construct outside language. In aggregate the elements of this critique suggests the need for an essential elaboration of Personal Construct Psychology in the direction of language.

Although others preempt this elaboration by locating Personal Construct Psychology within the domain of hermeneutics, a detailed examination of Kelly's (1955) position highlights the tenuous nature of this compatibility. Despite this, given the hopes held for the elaboration of many of the principles that are embedded within Kelly's (1955) position in the direction of language, this thesis now turns towards the hermeneutic phenomenology of HansGeorg Gadamer. In so doing, this thesis assumes that language is the missing medium within the Psychology of Personal Constructs. The inextricability of language from being as contended by Gadamer will thus provide a theoretical position from which to re-envision Kelly's core principles and provide a foundation for the development of a new theoretical position. This would be a position for catching a glimpse of the 'inner outlook' of the individual person for qualitative research. ${ }^{69}$

\footnotetext{
${ }^{69}$ As noted previously in Chapter Three, Kelly (1955) is open to, and in fact, welcomed the elaboration of his theoretical position. More recently, Holland, Neimeyer, Currier and Berman (2007) on the topic of theoretical elaboration of Personal Construct Psychology suggest that "even its adherents note that it may risk extinction if it remain an insular collection of radical visionaries" (p. 94). In so doing, these authors support the elaboration that is being proposed by this thesis.
} 


\section{CHAPTER 5}

\section{LANGUAGE AS THE MISSING MEDIUM}

The previous chapter culminated in a proposition that we proceed 'as if' language is indeed the missing medium within The Psychology of Personal Constructs. This chapter turns towards the hermeneutic work of Hans Georg Gadamer who argues that language is the universal medium within which all understanding occurs and therefore that language is the essential condition of being. This chapter will provide an explication of Gadamer's hermeneutic work and in so doing will outline a philosophical foundation from which to re-envision the principles distilled from the Psychology of Personal Constructs.

\section{Language as Being}

In theorising the construct as having its being beyond the reach of language, the establishment of a realist position beyond anything more that a 'slippery', naïve, or simple realism is difficult to sustain within Kelly's work. Moreover, the disconnect between language and the construct problematises the reflexivity of the Personal Construct Psychology position and its use ultimately in the garnering of a 'inner outlook' for the purposes of qualitative research. By not situating the construct - as a structure of ontological primacy within language, Kelly fails to develop a stable foundation from which a person can 'evolve' or 'work on' his or her constructions in the process of abstraction. In so doing, the notion of the construct as a genuinely ontological structure is open to skepticism. On the basis of this argument, and the view that several of Kelly's tenets will be legitimised and matured when elaborated in the direction of language, a turn is made toward the hermeneutic work of Hans Georg Gadamer.

This thesis will provide a thoroughgoing consideration of the way Gadamer (1960/2003) delineates language as the genuine medium of being human. This will establish a foundation from which to re-envision and subsequently overcome the challenges levelled at the Psychology of Personal Constructs, and in so doing will provide a strong theoretical foundation for qualitative research. However, an initial consideration of the way in which Gadamer's work is situated within the wider philosophical argument is essential. 


\section{Locating Gadamer}

Although the theoretical shifts made by Wittgenstein between the Tractatus LogicoPhilosophicus and Philosophical Investigations epitomise the Linguistic Turn, another major philosophical movement - Hermeneutics - cogitates in the same direction. Through a sustained critique of the subordination of language that can be seen in Kant's work, the Continental tradition, including thinkers such as Hamann, Herder, Humboldt, Schleiermacher, Dilthey, the later Heidegger ${ }^{1}$ and Gadamer, provide a united front against conceptions of the merely designative capacity of language. Instead, these proponents suggest that a philosophy that adheres to epistemological procedures and method, in an effort to mirror the object for the subject, neglects the possibilities of an expressive or hermeneutic disclosure of the world. Therefore hermeneutic thought, in contrast to the designative account of language that it seeks to critique, takes language to be disclosing of the world, and not therefore merely a tool for reflecting the world.

The publication of Gadamer's Truth and Method (1960) was a decisive moment in philosophical thought. Arguably Gadamer's entire treatise in Truth and Method can be considered a response to or critique of naïve or designative views of language used as a form of scientific rationalism. Thus within the pages of Truth and Method is an extensive theoretical engagement that paves the way for Gadamer to develop a theoretical reflection upon understanding, a position from which his most defining concepts arise. His typically critical review of modern aesthetic and historical consciousness provides a foundation from which he begins to overcome the alienation of the subject from the object of understanding. This is an alienation that he believes has been inherited from Enlightenment thought. Upon this foundation he mounts his philosophical hermeneutics ${ }^{2}$ and ontology of language. Gadamer divides Truth and Method into three major sections: The truth-value of art, truth as conceived in the human sciences, and his own iteration of the ontological nature of hermeneutics. Despite the primacy of the first two sections of Truth and Method to the development of Gadamer's own theoretical position, it is the third section that is most pivotal for the development of this thesis. Nevertheless certain key ideas from earlier sections will be drawn upon in the forthcoming consideration of Gadamer's hermeneutic approach.

\footnotetext{
${ }^{1}$ Gadamer is openly indebted to the work of those who have gone before him. Although a thoroughgoing consideration of the theoretical lineage of hermeneutic thought is outside the realm of this thesis, within the pages of Truth and Method one will find a detailed discussion on this topic. In brief, Schleiermacher universalised the notion of hermeneutics for Gadamer, while Dilthey influenced the historicity of Gadamer's thought. Heidegger's influence is particularly prolific and deserves special consideration in this chapter.

${ }^{2}$ Interestingly this was to be the original title of Truth and Method until a publisher suggested otherwise.
} 
Before unpacking the way in which Gadamer achieves a conceptualisation of language as the medium for being human, it is important to first appreciate the way he proposes an inextricable connection between the subject and the object of understanding through his notion of historicity. The following sketch provides a foundation for a later appreciation of his notion of language as the fundamental ontological structure.

\section{Historicity of Understanding}

As noted above, Gadamer argues that philosophical thought - as

Geisteswissenschaften - has inherited from the Enlightenment a separation between subject and object, knower and known. An alienation of the person from the world. This separation of subject and object represents a problem that traditional science - Naturwissenschaften has been assumed to have overcome through the application of methodological principles. ${ }^{3}$ However, for Gadamer (1960/2003), this separation represents a philosophical and transparent falsehood perpetuated by the proponents of objectivism. In seeking to show that a person is in fact always connected to that which is to be understood, Gadamer looks to human historicity to begin to apprehend an understanding of human understanding itself and, in so doing, overcome this falsehood. Gadamer turns toward a consideration of the experience of art as an exemplar.

\section{Experience of Art as Play}

Gadamer introduces the term 'aesthetic consciousness'. Inherent within this term is a suggestion that the typical consideration of visual arts situates artwork itself as having no ultimate contribution to society beyond perceptually pleasing or aesthetic qualities. That is, art provides pleasure devoid of the possibility of providing knowledge. For Gadamer (1960/2003), this view merely perpetuates the separation of subject and object. He goes on to argue that if a person is able to view art in terms of it representing a world, then art becomes more than simply a certain kind of sense perception. It becomes knowledge.

Gadamer goes on to contend that an experience with a work of art is an experience with the world and therefore is an experience of truth. Nonetheless, this truth is indeed a truth that is free from the procedures of methodological science. Thus, although this truth is not a scientific truth, for Gadamer it is not an inferior truth either. It is rather a truth about a person's Self. A person who sees art as representing a world is able to see the world

\footnotetext{
${ }^{3}$ Championing reason as the bastion of truth, Enlightenment thought firmly located notions of truth within the stronghold of scientific method. In other words, any claim to truth made outside of the rigorous application of scientific method could have no claim at all. Gadamer's (1960/2003) challenge could be considered a look at the possibilities of human understanding once freed from the limits of technical control and method.
} 
differently, in a new light, as if for the first time. This allows that person to be transformed into the world presented by the art. This transformation occurs through a mediation between his or her own understandings and those that are presented in the art, allowing that person to achieve a new or different understanding from that which he or she began with. Here a passage from Gadamer (1960/2003) is explanatory.

The pantheon of art is not a timeless present that presents itself to a pure aesthetic consciousness, but the act of a mind and spirit that has collected and gathered itself historically... Since we meet the artwork in the world and encounter a world in the individual artwork, the work of art is not some alien universe into which we are magically transported for a time. Rather we sublate the discontinuity and atomism of isolated experience in the continuity of our own existence. For this reason, we must adopt a standpoint in relation to art and the beautiful that does not pretend to immediacy but corresponds to the historical nature of the human condition. (p. 97)

Here Gadamer suggests that the person reaches toward art from the perspective provided by his or her historicity. In fact, it is a person's historicity that provides the possibility that art can come to understanding at all. Gadamer (1960/2003) describes this experience in terms of an Erfahrung, as an experience that is mediated by historicity (p. 102). Here, when we enter a world of art, we come to understand our self and our world through the art. Seeking to unpack further the way in which a person is always bound to the thing that is to be understood from the perspective of his or her historicity, Gadamer introduces a notion that is central to much of his work - play.

A look at the meanings implied by the German word spiel provides greater clarity to Gadamer's use of the term play. Originally the term spiel meant 'to dance' or 'a dance' and embodied for Gadamer the inherent reciprocity between those engaged in the dance and who are also always at the same time separate. This same reciprocity represents for Gadamer $(1960 / 2003)$ the relation of the person with art.

When we speak of play in reference to the experience of art, this means neither the orientation nor even the state of mind of the creator or of those enjoying the work of art, nor the freedom of a subjectivity engaged in play, but the mode of being of the work or art itself. (p. 101)

Gadamer suggests that a work of art does not have an understanding that is simply transmitted into the consciousness of the viewer. Rather, for Gadamer (1960/2003), the artwork and the 
appreciator are drawn together in a "to-and-fro movement that is not tied to any goal that brings it to an end" (p. 103). Instead, art finds its expression only in the experience of the audience, where both the art and the audience attend to the meaningfulness of the work. Thus, in the 'dance' of the experience of art, a person comes to recognise what it is that he or she already knows, an understanding that Gadamer describes as a self-understanding. That is, the person recognises and comes to know his or her Self. It is this self-understanding that Gadamer (1960/2003) describes as a truth. Although he recognises that the experience of art is but one experience, he believes it epitomises human experience of the world more generally. Therefore for Gadamer (1960/2003) the use of the term spiel adequately captures the 'to and fro' nature of human experience more generally, as a movement where the subject and the object always coalesce in understanding.

Just as his exploration of aesthetic consciousness emphasises the way in which a person is never separated from the experience of art, it also provides an exemplar of the way that each person is indeed never alienated from the world of his or her experience. Instead this person is always a part of the world through a relationship that adopts the form of play. It follows, therefore, that for Gadamer (160/2003) any epistemological separation of subject and object enacts a falsehood of an aesthetic consciousness. He accentuates this falsehood by underscoring how each person is never separate from his or her history. This position is embodied by Gadamer's notion of Wirkungsgeschichtliches Bewußtsein of which an elaboration follows.

\section{Wirkungsgeschichtliches Bewußtsein}

Arguably one of the defining features of Gadamer's work is his notion of Wirkungsgeschichtliches Bewußtsein. Eluding a complete English translation, this phrase is most frequently translated as "Consciousness in which history is ever at work" (Palmer, 1969, p. 191), or by Gadamer (1960/2003) as "historically effected consciousness" (p. 340). Gadamer seeks to emphasise that a person's historicity is not something that one can simply remove him or her self from. Instead, a person's consciousness is at every moment affected by historicity. In other words, the primacy of historicity means that there is no possibility of a 'historicity free' position. It is not possible to retreat to a position that is free from our historicity in order to stand-back from and reflect upon that historicity. In considering the possibility that we can reflect upon our historicity, Gadamer (1960/2003) suggests that "the fact that it cannot be completed is due not to a deficiency in reflection but to the essence of the historical being that we are. To be historically means that knowledge of oneself can never be complete" (p. 302 emphasis in original). Gadamer goes on to suggest that historicity therefore places limitations upon human consciousness and the possibilities of what can be 
understood. In other words, having our being within historicity means that we cannot step outside of it to a position of objectivity. Rather we always 'light-up' the situation from within it. As a means of unpacking further the limitations of historicity upon human consciousness and the subsequent possibilities of understanding, Gadamer develops the notion of 'horizon'. A horizon stands for everything that can be seen from a particular vantage point. Before exploring this notion of horizon further, however, it is important to first appreciate other features of the relationship that one has with the world in understanding.

By examining the way in which a person's historicity is mediated with the present as the very condition of understanding itself, Gadamer (1960/2003) rehabilitates several features of human understanding that had previously been exiled by Enlightenment and modern thought (Lawn, 2004). Gadamer's rehabilitation of these ideas further reveals the centrality of Wirkungsgeschichtliches Bewußtsein and the inextricability of historicity from human understanding. His conceptualisation of the circle of understanding provides a most suitable entrée to an appreciation of these rehabilitated features. ${ }^{4}$

\section{Hermeneutic Circle of Understanding}

Borrowing heavily from the work of Heidegger, Gadamer reinstates the notion of a circularity of understanding. Seeking to do justice to the historicity of human understanding he thus adopts the imagery of the circle to emphasise that understanding always involves a constant mediation between past and present. Born of a rule from ancient rhetoric, ${ }^{5}$ Gadamer (1960/2003) suggests that "the movement of understanding is constantly from the whole to the parts and back to the whole" (p. 291). For Gadamer, then, understanding begins first and foremost with anticipations, structures that he describes as prejudices, and that provide entrée to this circle of understanding as well as the possibility of this 'whole-parts-whole' mediation.

\footnotetext{
${ }^{4}$ Gadamer is clear that he begins from the position that Heidegger developed. In so doing, Gadamer also begins with Heidegger's interpretation of the circle of understanding (Gadamer, 1960/2003). For Heidegger the circle of understanding provided figurative representation for his ontological conception of human understanding. Heidegger proposed that there can be no interpretation without prior understanding and therefore every interpretation is preceded by another interpretation (Heidegger, 1926/2005). To capture this idea Heidegger used the symbolism of the circle as a metaphor. This circularity is epitomised by Heidegger's notion of Dasein as the essential movement of what he described as being in the world (Heidegger, 1926/2005). Although Gadamer begins with Heidegger's conception of the circle of understanding, Gadamer goes on to proposes his own circular conception of understanding and works to protect it from the challenges levelled against Heidegger's circle. Grondin suggests that Heidegger sought to avoid describing Dasein as a circle because of the risk of being seen to represent a "logical-vice" (2003, p.79), whereby an interpretation is merely the confirmation of an understanding that is bestowed in advance. However, Gadamer established that the prejudice is a precondition of understanding. Here all understanding emerges in the light of these prejudgement or prejudices (Gadamer, 1960/2003. Thus for Gadamer the circle comes to represent the possibilities of understanding rather than its completion.

${ }^{5}$ This rule suggests that the constant task of understanding is the revision of our anticipations in the light of greater knowledge of the parts.
} 


\section{The Prejudice}

Here Gadamer rehabilitates a conceptualisation of the prejudice from its connotations as a false and irrational thought, a position he argues is also inherited from the Enlightenment, ${ }^{6}$ and reinstates it in terms of its earlier meaning. According to Gadamer (1960/2003), the term 'prejudice' historically carried the meaning of "a judgement rendered before all the elements that determine a situation have been finally examined" (p. 270). It is precisely this notion of prejudgement that is important for Gadamer. He envisages the prejudice as productive for describing how it is that a person's consciousness is always effected by historicity and therefore for underscoring his notion of Wirkungsgeschichtliches Bewußtsein.

Gadamer (1960/2003) suggests that "If we want to do justice to man's finite, historical mode of being, it is necessary to fundamentally rehabilitate the concept of prejudice and acknowledge the fact that there are legitimate prejudices" (p. 277). As such he situates the prejudice as the essential anticipatory structure that provides entrée to and sustains the circular movement of understanding, a movement that is always a mediation between past and present. Given that the way in which the prejudice provides entrée to the circle of understanding will be explored in more detail shortly, it is important to apprehend more fully the nature of the prejudice itself. For this reason a closer look at tradition is valuable.

It is perhaps the notion of tradition that best represents what Gadamer intended by his idea of a prejudice. Tradition, for Gadamer, is all that is handed down through being present, or through one's being-there in the world. Importantly, though, this handing down is not intended to represent a collection of facts that can simply become an object of consciousness in the form of reflection, and can then be simply passed over to another person like an object or a gift. Rather, tradition is like a flowing stream in which one moves and which participates in every act of understanding. Here, then, each person is always situated within a tradition and through that tradition is able to meaningfully come to understand the world. Tradition, therefore, has for Gadamer (1960/2003) a 'silent authority'. However, this authority is not to be confused with a loss of self-determination. Rather, for Gadamer (1960/2003), the authority of a tradition is only ever those aspects that are preserved by being cultivated and perpetuated, which is more than simply a 'handing- down'.

\footnotetext{
${ }^{6}$ Taking the position that all understanding inevitably involves pre-understanding, Gadamer (1960/2003) suggested, "there is one prejudice of the Enlightenment that defines its essence: the fundamental prejudice of the Enlightenment is the prejudice against prejudice itself, which denies tradition itself" (p. 270). Grondin (2003) describes Gadamer's sentiments as ingenious and suggests that the statement "presupposes that there are prejudices prejudicial to the understanding and that the prejudice against prejudices is a part of it" (p. 84).
} 
The fact is that in tradition there is always an element of freedom and of history itself. Even the most genuine and pure tradition does not persist because of the inertia of what once existed. It needs to be affirmed, embraced, cultivated. It is, essentially, preservation, and it is active in all historical change. (p. 281)

What Gadamer concerns himself with here is the notion of tradition as a prejudice. Tradition is what makes our present being possible; it is what is at work within us, beyond our knowledge of it (Grondin, 2003). Thus Gadamer (1960/2003) contends that a person is always situated within tradition, not as something other, but rather as something that is "always part of us, a model or exemplar, a kind of cognizance that our later historical judgement would hardly regard as a kind of knowledge" (p. 282). For Gadamer (1960/2003) then, tradition represents "a prior condition of understanding" (p. 309). In so doing, tradition provides Gadamer with an opportunity to highlight the way in which human understanding is always and only possible against a backdrop of anticipatory interpretations or prior conditions of understanding.

With this in mind, a return to the notion of the prejudice can now be made. For Gadamer these anticipatory conditions of understanding - exemplified by tradition - are a person's prejudices. Conceptualised in terms of 'a light', it is by the light provided by these anticipations that a person comes to see the world. Thus, a person's prejudices form the standpoint that he or she inhabits or the possibilities that he or she has available to them for envisaging the world. It is clear through the example of tradition that, for Gadamer, all understanding emerges entirely in the light of our anticipations or our prejudices. ${ }^{7}$ Here a turn can now be made to a further elaboration of the notion of horizon.

\section{The Horizon}

Conditioned by a person's prejudices, a horizon embodies the possibilities available for the person to envision the world. A horizon therefore encompasses all that can be seen from a particular vantage point. As with everyday usage, it also implies that the person can move beyond simply what is nearest to him or her in order to expand his or her horizon and therefore include greater possibilities of understanding. The following passage from Gadamer (1960/2003) is facilitative of appreciating this concept.

\footnotetext{
${ }^{7}$ Grondin (2003) captures the way in which for Gadamer the prejudice is the most primordial and anticipatory structure of the movement of human understanding: "For Gadamer all understanding emerges entirely in the light of anticipations (which we can call 'prejudices') so much and so well that the correction of a prejudice, shown to be illegitimate, is always only made in light of a new anticipation which replaces the previous one" (p. 85).
} 
The concept of "horizon" suggests itself because it expresses the superior breadth of vision that the person who is trying to understand must have. To acquire a horizon means that one learns to look beyond what is close at hand - not in order to look away from it but to see it better, within a larger whole and in truer proportion. (p. 305)

Being conditioned by prejudices, a person's interpretations or ideas about the world are therefore always born of his or her historical situation. It follows, then, that our horizon always emanates from a particular position. This is not an unconditional or dogmatic position. Instead this understanding marks a beginning point that is always open to expansion to a new or broader horizon.

In order to emphasise the importance of the notion of horizon and its centrality to an elaboration of Wirkungsgeschichtliches Bewußtsein, Gadamer takes what he believes to be the commonly accepted view of how it is that one person comes to understand another person. He suggests that at the foundation of the traditionally held view is a belief that one person merely adopts the point of view of the other person. In other words, one person steps into the horizon of the other. In so doing, this person moves from the familiar - his or her own horizon - to the alien - the other person's horizon. Gadamer, however, suggests that this is not fully representative of the process of understanding itself and goes on to contend that such a conception is still beholden to a naïve view about the notion of historicity. ${ }^{8}$ Gadamer argues that the idea of simply stepping into the horizon of another person must assume the possibility that a person is able to abandon his or her historicity or horizon. This however is incompatible with the notion of the prejudice as the pre-condition of understanding. For this reason Gadamer goes on to suggest that understanding always involves a coalescence of horizons. Here each person's horizon - as the embodiment of the totality of that person's historicity - provides the anticipatory possibilities of what can be understood of the other person. Thus it is only from within a person's own horizon that he or she has the possibility of bringing that which is other to meaningful understanding. For Gadamer, it is a person's historicity that therefore provides the horizon of the past that then coalesces with what he describes as the horizon of the present. This coalescence of horizons is captured within the following passage of Gadamer's (1960/2003).

\footnotetext{
${ }^{8}$ It should be noted that this position is inconsistent with the indissolubility of historicity from understanding that Gadamer (1960/2003) is attempting to establish. He goes on to suggest that the protecting of one's horizon from having any interplay with that which is other, withholds from that other the possibility of being true. In other words, beyond our horizon that other cannot have the possibility of being meaningfully understood and thus cannot be confirmed or understood differently and cannot therefore come to have a lasting effect as a truth in the historicity of an individual person.
} 
When our historical consciousness transposes itself into historical horizons, this does not entail passing into alien worlds unconnected in any way with our own; instead, they together constitute the one great horizon that moves from within and that, beyond which the frontier of the present, embraces the historical depths of our self-consciousness. Everything contained in historical consciousness is in fact embraced by a single historical horizon. (p.

Gadamer uses the term fusion to represent this coalescence or interplay between the horizon of the present and the horizon of the past. ${ }^{9}$ In fact, Gadamer goes on to establish that it is in this moment of fusion that understanding itself occurs. In this fusion a person's projected historical consciousness is simultaneously superseded, giving way to a different horizon or a different vantage point as a result of understanding. ${ }^{10}$ Gadamer (1960/2003) indicates that central to this fusion is the role of Wirkungsgeschichtliches Bewußtsein and that this notion operates to emphasise the inextricability of subject from object in understanding.

To bring about this fusion in a regulated way is the task of what we called historically effected consciousness. Although this task was obscured by aesthetic-historical positivism following on the heels of romantic hermeneutics, it is in fact the central problem of hermeneutics. (p. 307)

Despite the way that the coalescence of horizons embodied by the idea of understanding as a fusion overcomes the alienation of the knower from the known, the problem he alludes to is the nature of the understanding that this fusion actually is, that is, understanding as application.

\section{Application}

In order to rehabilitate a previously lost notion of application, Gadamer (1960/2003) turns towards the legal and theological traditions in hermeneutics that he believes embodies a recognition or preservation of application as being central to understanding. ${ }^{11}$

\footnotetext{
${ }^{9}$ The notion of a 'fusion of horizons' will be taken up at a later stage in this chapter. Suffice to say here that according to Gadamer's Wirkungsgeschichtliches Bewußtsein, it is a philosophical faux pas to suggest that we merely step into the horizon of another and abandon the structures that provide the very condition of our understanding.

${ }^{10}$ Gadamer (1960/2003), establishes that although the horizon of the present and the horizon of the past exist as distinct from each other, "understanding is always the fusion of these horizons supposedly existing by themselves" (p. 306).

${ }^{11}$ Gadamer (1960/2003) reviews the history of the development of hermeneutics and indicates that it was once divided into three areas or essential capacities of understanding or subtilitas - understanding
} 
This [legal and theological hermeneutics] implies that the text, whether law or gospel, if it is to be understood properly - i.e., according to the claim it makes - must be understood at every moment in every concrete situation, in a new and different way. Understanding here is always application. (p. 309)

Here one takes something from the past and brings it to life anew in the present by way of application. Using the example of legal hermeneutics, ${ }^{12}$ Gadamer (1960/2003) suggests that "to be faithful to the spirit of justice intended by the law itself is to adapt its application to the particular circumstance of a precise case" (p. 327). Here the judge does not simply know the law and place it upon a particular case, but must rather remain open to the claim that the law makes and apply the law to the needs of the situation. In so doing, the judge interprets the meaning of the law and applies it to the needs of a present case in such a way that it allows the law to speak to the citizens.

In terms of Wirkungsgeschichtliches Bewußtsein then, Gadamer's reference to application acknowledges that understanding does not represent a domination over a thing that is to be understood. Understanding rather always involves an interpenetration of past and present. In other words, a person does not know something and apply it as a universal or as a given. Instead, for Gadamer, application highlights that each person functions like a judge and applies one's understandings - that person's prejudices - that originate from his or her Wirkungsgeschichtliches Bewußtsein, bringing those understandings 'into play' through application. This play of past and present brought about by the application of a prejudice always reaches towards a fusion of horizons. Taken this way, application always has a productive rather than reductive effect in elaborating our understanding. A consideration of Gadamer's notion of experience is exemplary of this.

\section{Experience as Experience of Historicity.}

The culmination of Gadamer's argument concerning the establishment of a Wirkungsgeschichtliches Bewußtsein and critique of naïve objectivism can be found in his conceptualisation of human experience as experience of one's historicity. Here he expressed

(subtilitas intelligendi), interpretation (subtilitas explicandi), and application (subtilitas applicandi) ( $\mathrm{p}$. 307). While Gadamer, identifies that understanding and interpretation became united in post-Romantic hermeneutics - most notably in the work of Schleiermacher - he believes that the subsequent emphasis upon these tenets left little room for a consideration of application within the sphere of understanding. ${ }^{12}$ As already noted, Gadamer also turns to the theological domain as a means of emphasising the notion of application. He suggests that although the proclamation is genuinely concretised in preaching, much like the verdict of a judge, the proclamation does not carry the same dogmatic authority as the judgement imposed by the judge. The power of the proclamation is not decided by the thoughts of the preacher, but rather by the power of the word itself. For a more complete exploration of Theological hermeneutics as exemplary, see Truth and Method (2 ${ }^{\text {nd }}$ Ed.), pp. 330-341. 
his dissatisfaction with the prevailing epistemological conceptions of experience as being merely a perceptual act, an accumulation or collection of conceptual data. For Gadamer such approaches are obligated to the confirmation of experience, where experience can only become valid by not being disconfirmed by new experience. Gadamer, however, suggests that such an objectification of experience fails to recognise the productive value of experience that is disconfirming. So much is this the case for Gadamer (1960/2003) that he suggests "experience in the genuine sense is always negative" (p. 353). It is important to appreciate that Gadamer is not discrediting the possibility of situations that are confirming. Rather he is suggesting that confirmation does not represent experience per se.

The notion of experience as confirmation is merely a confirmation of something that a person already possesses. Confirmation therefore provides at best an encounter with that person's historicity and subsequently a realisation of the degree to which the application of his or her prejudices are adequate at anticipating the thing to was to be understood. The following passage from Gadamer (1960/2003) is exemplary of this discussion.

It is no longer a new experience when it is repeated and confirmed. When we have had an experience, this means we possess it. We can now predict what was previously unexpected. The same thing cannot again become a new experience for us; only something different and unexpected can provide someone who has experience with a new one. (p. 353)

In light of this, Gadamer (1960/2003) contends that "every experience worthy of the name thwarts an expectation" (p. 356), and therefore experience by its very nature is negative. This negativity can be captured in terms of being 'pulled-up' short by our anticipations. Being pulled-up short occurs when a person recognises that his or her prejudices are relatively ineffective in anticipating the thing that is to be understood.

It is important to appreciate, however, that for Gadamer this implicit negativity carries with it a degree of productivity. That is, in being 'pulled-up short' by our prejudices or anticipations, we return all the more to our own historicity. In so doing we come to recognise what was not adequately understood of the thing to be understood in the first instance, while also coming understanding that thing better or differently. The following passage from Gadamer (1960/2003) is explanatory of this.

Thus the negativity of experience has a curiously productive meaning...we cannot, therefore, have a new experience of any object at random, but it must 
be of such a nature that we gain better knowledge through it, not only of

itself, but of what we thought we knew before - i.e., of a universal. (p. 353)

Gadamer's (1960/2003) elucidation of experience as productively negative therefore implies a consideration of experience as something that surpasses what we already have. Thus, for Gadamer (190/2003), "the truth of experience always implies an orientation toward new experience" (p. 355). ${ }^{13}$ Here he is suggesting that in order for a person to have an experience, then he or she must have an orientation towards openness. Implicit within this notion of openness is a readiness for experience, a readiness for a person to bring into play his or her prejudices in the expectation of having them surpassed as part of coming to understand a thing differently or better. Thus, in drawing to its maximum the notion of Wirkungsgeschichtliches Bewußtsein, Gadamer (1960/2003) suggests that "genuine experience is experience of one's own historicity" (p. 356), it is an understanding of what we know and indeed what we do not know.

\section{Understanding as Understanding One's Self}

Gadamer's elaboration of the structure of human experience and understanding embodied within his notion of Wirkungsgeschichtliches Bewußtsein operates to underpin the way in which for him the subject and object are inextricably connected in every event of understanding, where this connection has as its foundation a coalescence of past and present. His elaboration of the inherent negativity of experience highlights that it is experience in the genuine sense that sustains understanding, where this understanding is ultimately an understanding of one's self. Understood in this way, then, understanding is no longer a cognitive or methodological process, instead it is inextricable from the flowing stream of being. In other words, for Gadamer (1960/2003), understanding is no longer merely an operation, it is "the original characteristic of the being of human life itself" (p. 259). ${ }^{14}$ To capture this notion more completely, Gadamer (1960/2003) turns again to the work of Heidegger and suggests that "understanding is...the original form of the realization of Dasein, which is being-in-the-world" (p. 259). Gadamer's open indebtedness to the notion of Dasein provides a suitable justification for a brief sketch of this key point.

\footnotetext{
${ }^{13}$ Gadamer (1960/2003) suggests that an experienced person "does not consist in the fact that someone already knows everything and knows better than anyone else. Rather, the experienced person proves to be, on the contrary, someone who is radically undogmatic; who, because of the many experiences he [sic] has had and the knowledge he has drawn from them, is particularly well equipped to have new experiences and to learn from them" (p. 355).

${ }^{14}$ For Grondin (2003), Gadamer's recognition of understanding as the fundamental aspect for being human represents a significant philosophical "unblocking" (p. 75) that enabled the remainder of Gadamer's philosophical position to be established.
} 


\section{Dasein as Exemplary of the Ontologisation of Understanding}

Reaching beyond a conceptualisation of understanding as merely a method of deciphering, or as a special case of the methodological sciences, Heidegger established that understanding is in fact the very form of human 'being-in-the-world' or Dasein. For Heidegger, understanding is the way in which humans exist within the world. His conception of Dasein also captures the way that understanding cannot simply be the product of accumulated facts that a person then applies to the thing that is to be understood. It is instead a process that requires the activity of a reflective consciousness. Indeed, Dasein is the understanding that emerges from a being-there within the world. It represents a movement born of the stream of historicity in which we move. Dasein is the ontologically grounded comportment of being human. The following passage from Heidegger (1926/2005) describes this conception of Dasein.

Dasein is an entity which, in its very Being, comports itself understandingly towards that of Being. In saying this, we are calling attention to the formal concept of existence. Dasein exists. Furthermore, Dasein is an entity which in each case I myself am...Dasein stretches along between birth and death (p. $78,425)$.

With this in mind, Gadamer (1960/2003) suggest that "the structure of historical understanding appears with its full ontological background" (p. 261) through the notion of Dasein. Heidegger's conception of Dasein highlights that understanding can never be a mastery over something (noesis noeseos). Instead, it recognises the finitude of the capacities of human understanding. The ontologisation of understanding in Dasein leads Gadamer (1960/2003) to contend that "All such understanding is ultimately self understanding ...thus it is true in every case that a person who understands, understands himself [sic] (sich versteht), projecting himself upon his possibilities" (p. 260). Through conceiving of understanding as the basic mode of being and not in terms of correctness, Heidegger thus liberated understanding from the 'strait-jacket' of epistemological thought (Grondin, 2003). In so doing, he provides the necessary theoretical manoeuvre required for Gadamer to establish his own elaboration of human understanding through his philosophical hermeneutics.

\section{Understanding as Interpretation}

Acknowledging Heidegger's conception of Dasein and ontologisation of understanding as a theoretical opening for his own work, Gadamer explores the type of knowledge that results from such an approach. For Gadamer this understanding is most congruent with the notion of interpretation. It is important to recognise that interpretation for 
Gadamer (1960/2003) is not an occasional post-facto supplement to understanding per se, rather for him "understanding is always interpretation, and hence interpretation is the explicit form of understanding" (p. 307). It is important to appreciate that interpretation is not merely what we superimpose upon a thing to be understood. On the contrary, Gadamer (1960/2003) suggests that the better an interpretation is the less it is superimposed and the more the thing that is to be understood is effaced by it. Importantly this elucidation is not merely a naïve objectivity captured in a different guise, whereby the object of understanding comes to meaning merely by the rightness of an interpretation. Instead here an interpretation is correct if it escapes notice as an interpretation at all, allowing the thing to be understood or to 'speak' for itself. It follows, then, that only those interpretations that fail to adequately bring the thing to be understood to understanding are recognised as being superimposed and therefore fail as interpretations in the Gadamerian sense.

In order to emphasise what is meant by the notion of an interpretation effacing itself as an interpretation, Gadamer (1960/2003) turns towards the movement of a conversation conducted in two different languages. Here he emphasises the centrality of translation. In such a conversation the translator must translate the meaning to be understood into the context in which the other person lives. However in the following passage Gadamer (1960/2003) is clear that this is not the production of an arbitrary meaning.

This does not, of course, mean that he [sic] is at liberty to falsify the meaning of what the other person says. Rather, the meaning must be preserved, but since it must be understood within a new language world, it must establish its validity within it in a new way. Thus every translation is at the same time an interpretation. We can even say that the translation is the culmination of the interpretation that the translator has made of the words given him. (p. 384)

Thus despite the faithfulness of the translator to the original, the fundamental gulf between the two languages cannot be closed completely. Gadamer (1960/2003) suggests that "Where a translation is necessary, the gap between the spirit of the original words and that of their reproduction must be taken into account. It is a gap that can never be completely closed" (p. 384). Nonetheless, in seeking to bridge this gap between the original and its reproduction, the translator makes difficult decisions about what elements of the thing to be understood to emphasise and which to play down or entirely suppress. Here Gadamer (1960/2003) 
recognises that in interpretation, "even if it is a masterly re-creation it must lack some of the overtones that vibrate in the original" (p. 386). ${ }^{15}$

In returning to Gadamer's consideration of understanding as interpretation - adopting the essential structure of translation - it is clear that any interpretation must 're-awaken' the meaning of the thing that is to be understood. This re-awakening is the task of allowing the thing to be understood to speak a meaning that can be apprehended in the here and now of being. However the very nature of human historicity leads Gadamer (1960/2003) to suggest that no single 're-awakening' is the correct one.

To try to escape from one's own concepts in interpretation is not only impossible but manifestly absurd. To interpret means precisely to bring one's own preconceptions into play so that the text's meaning can really be made to speak for us...Thus interpretation must find the right language if it really wants to make the text speak. There cannot therefore, be any single interpretation that is correct 'in itself', precisely because every interpretation is concerned with the text itself. The historical life of tradition depends on being constantly assimilated and interpreted. An interpretation that was correct in itself, would be a foolish ideal that mistook the nature of tradition. Every interpretation has to adapt itself to the hermeneutical situation to which it belongs. (p. 397)

Here the earlier emphasis upon application is writ large. To understand a text always means for Gadamer (1960/2003) to apply it to one's self and "even if it must always be understood in different ways, it is still the same text presenting itself to us in these different ways" ( $p$. 398). What is right in an interpretation is therefore not what 'is right' per se in an objective sense, but rather what is right as embodied by a 'fusion of horizons'. Thus the point at which an interpretation allows the thing to be understood - and thereby effaces itself as an interpretation at all - is that point where a person can be borne along by his or her understanding in the ongoing movement of being. However the coalescence of horizons that is implicit within Gadamer's elucidation of understanding as interpretation follows a dialogical model, a model toward which a turn is now made.

\footnotetext{
${ }^{15}$ One cannot help think about how this may relate to Truth and Method. That is, the degree to which some of the vibrations that resonate in the original - German version - are perhaps lost in the English translation. It is worth noting on this, that unlike many translations that are carried out posthumously, Truth and Method was not. Gadamer lived to be 102 years of age and was a consultant in the process of translation itself. Moreover, where there is no 'ready at hand' English translation the German usage has remained.
} 


\section{Dialogical Mode of Understanding}

Through his elaboration of Wirkungsgeschichtliches Bewußtsein Gadamer is clear that a person is always affected by his or her historicity. So much so that this historicity is inextricable from the movement of being itself, a movement that Gadamer suggests is always toward understanding. Understanding for Gadamer always originates in a coalescence, a movement or 'play' between a person's historicity and that which is to be understood. It is this play that Gadamer believes is well served by the notion of a dialogue. This is because a genuine dialogue embodies an essential openness on the part of the person who seeks to understand a thing. In a dialogue, one person does not seek to argue the other person down or to find a weakness, but rather considers the weight of the opinion of the other person. For Gadamer (1960/2003), dialogue is "not the art of arguing (which can make a strong case out of a weak one) but the art of thinking (which can strengthen objections by referring to the subject matter)" (p. 367). From this position Gadamer (1960/2003) turns to the 'conduct' of a conversation in order to extend further his consideration of the dialogical nature of understanding. ${ }^{16}$

A conversation is not something that a person conducts, but rather something that one finds him or herself in. In fact it is the spirit of the conversation that leads the person(s) along, in such a way that the thing that ultimately emerges through the conversation cannot be known in advance. Within a conversation, then, one person does not attempt to transpose him or her self into the other person in order to relive the experiences (Erlebnisse) of that person. Here we must recall that a person always understands a thing to be understood from within his or her own worldview (Erfahrung). To have a genuine dialogue then, one person does not adopt or accept like a gift that which the other person is saying. Instead a person always allows that which is being said to have 'play' or to coalesce with his or her historicity or worldview in order to reach an understanding. A passage from Gadamer (1960/2003) is explanatory of this coalescence.

Conversation is a process of coming to an understanding. Thus it belongs to every true conversation that each person opens himself [sic] to the other, truly accepts his point of view as valid and transposes himself into the other to such an extent that he understands not the particular individual but what he says. What is to be grasped is the substantive rightness of his opinion, so that

\footnotetext{
${ }^{16}$ It is interesting that although Gadamer does provide a specific elaboration of the notion of dialogue, if one was to look up the word 'dialogue' in the index of Truth and Method, Gadamer directs the reader to see Conversation. Here it is clear that for Gadamer the notions of dialogue and of conversation are synonymous.
} 
we can be at one with each other on the subject. Thus we do not relate the other's opinion to him but to our own opinions and views... reaching an understanding in conversation presupposes that both partners are ready for it and are trying to recognize the full value of what is alien and opposed to them (pp. 385, 387).

Here Gadamer has highlighted that to understand (Verstehen) is ultimately to agree (sich verstehen mit) with somebody about a thing. Importantly, however, the person is not adopting the viewpoint of another person by way of a restructuring of his or her own historicity. ${ }^{17}$ Instead that person is in agreement with the well-foundedness of the thing that is under consideration. That is, if the interpretation of the thing itself is convincing - effaced as being an interpretation in dialogue - the participants within the dialogue are carried along by this well-foundedness. The following passage from Gadamer (1960/2003) captures this movement.

The interpreter's own thoughts too have gone into re-awakening the text's meaning. In this the interpreter's own horizon is decisive, yet not as a personal standpoint that he maintains or enforces, but more as an opinion and a possibility that one brings into play and puts at risk, and that helps one truly to make one's own what the text says. I have described this above as a 'fusion of horizons'. We can now see that this is what takes place in conversation, in which something is expressed that is not only mine or my author's, but common. (p. 388)

Gadamer has shown here that it is the dialogue that determines the movement toward understanding and not the participants. So much so that for Grondin (2003), "those who take part in a dialogue are not so much participants as those within whom the dialogue participates" (p. 127). Thus Gadamer has been able to highlight that through a common everyday occurrence - the conversation - and its implicit to-and-fro movement, a person can indeed achieve an understanding. ${ }^{18}$ Interlocutors simply 'fall' into conversation, conduct themselves without formal structure or systematised method and, focusing always on the subject matter that is placed before them, often arrive at no unanimous conclusion. Despite

\footnotetext{
${ }^{17}$ Gadamer here makes reference to Schleiermacher's work and his requirement that one seeking to understand reconstruct the historicity from which the original author originated. See Truth and Method pp. 184-199, 387-388.

${ }^{18}$ It must be remembered that understanding is not an end in itself, rather understanding always takes the form of degrees of understanding. It follows that if all understanding is language, then the refinement of language is the refinement of understanding. It is perhaps this notion that provides a means of capturing the breadth of understanding that can be seen across both socioeconomic and educational backgrounds.
} 
this, interlocutors can achieve a genuine understanding of that which is other. Although his consideration of the essential movement of the conversation follows logically what occurs between people, Gadamer believes the same movement of conversation is in fact emblematic of the hermeneutic understanding more generally. He suggests that at the foundation of dialogue is a movement that takes the essential structure of question and answer.

\section{Question and Answer as the Foundation of Dialogue}

It is clear that many things within the world cannot speak or engage in a conversation in the same manner as two or more people relating to each other. However for Gadamer these things - metaphysical or otherwise - must be made to speak by the person who is seeking to understand. That is, those things must become a Thou. For Gadamer (1960/2003) a Thou "is not an object; it relates itself to us" (p.358). A thing itself is not a Thou simply by being an object or something other, instead a thing becomes a Thou through the person making his or her horizon coalesce with the horizon of that thing. It is here that the centrality of questioning arises. In order, then, to undertake a focused consideration of a Thou, a closer look at the notion of a question from Gadamer's perspective is necessary.

A question brings into the open that about which an answer is not yet settled. In this way the question is central to the notion of experience as essentially negative and yet productive. That is, from a recognition of what it is that we do not know, a question originates which implies 'a will to know' a thing better or differently. Emanating from an experience of our Wirkungsgeschichtliches Bewußtsein, for Gadamer (1960/2003) a question therefore brings with it a recognition of our historicity in that "it implies the explicit establishing of presuppositions, in terms of which can be seen what still remains open" (p. 363). Posing a question therefore always implies a reaching towards that which is still to be settled of our understanding. In following this, the notion of question and answer reaffirms the finite nature of human understanding. That is, understanding is always conditioned by the questions that can be asked. However, we must recognise that it is always possible for us to ask questions in another way. In so doing, we can continue to understand the thing that is to be understood differently. In fact, as a function of this, our understanding of the thing can never be complete.

Gadamer is quick to acknowledge that questions can be asked rightly or wrongly. A wrongly asked question closes down further possibilities of understanding. And, in so doing, a wrongly asked question limits the ways that the thing to be understood can provide an answer. A rhetorical question for example provides a useful illustration of a question that fails to open up further possibilities of understanding. In this example, one asks a question to 
which the answer is not in dispute. Instead, the person asking is merely seeking to close down the discussion at a predetermined point. A legitimate question, on the contrary, would be one that allows the subject matter to be understood and to be opened up by the question in such a way as to enable a dialogue. In fact, for Gadamer (1960/2003), "the art of questioning is the art of questioning even further i.e., the art of thinking" (p. 367).

With this in mind, a person poses a question of the thing to be understood in terms of a 'is it a this' or 'is it a that' to which the thing to be understood is able to provide the answer. The centrality of the question to understanding is captured by Gadamer (1960/2003) in the following passage.

The question opens up possibilities of meaning, and thus what is meaningful passes into one's own thinking on the subject...to understand a question means to ask it. To understand meaning is to understand it as the answer to a question. (p. 375)

In light of this, then, questions that arise from a person's Wirkungsgeschichtliches Bewußtsein always reach toward a self-understanding. That is, the person seeks answers from the thing that is made to speak as a Thou. These answers ultimately shape one's Wirkungsgeschichtliches Bewußtsein for understanding in the future. Being able to pose a question therefore means that we have been able to recognise what it is of our own understanding or our Self that is in need of development. Through a coalescence with the thing - as a Thou - we are brought back all the more to our own understanding. This understanding is always a coalescence of past as Wirkungsgeschichtliches Bewußtsein, as our Self, with a present or the thing that is made to relate to us as a Thou, in a fusion of horizons.

\section{Language as The Medium}

The guiding idea of Gadamer's work thus far has been to emphasise that a person cannot establish an epistemological gap that warrants a claim to objectivity between him or her self and the thing to be understood. Instead, understanding in terms of Wirkungsgeschichtliches Bewußtsein always involves a coalescence of the past and present; of subject and object. This movement between past, present, subject and object is epitomised by the movement of the conversation and the logic of question and answer. This movement always reaches towards understanding as the essential mode of being, and always takes the form of a fusion of horizons. In the final section of Truth and Method, Gadamer (1960/2003) suggests that "the fusion of horizons that takes place in understanding is actually the achievement of language" (p. 378). The following passage emphasises the "uncanny nearness 
of language' that Gadamer (1960/2003) turns toward in propelling his hermeneutic elaboration further.

What language is belongs among the most mysterious questions that man [sic] ponders. Language is so uncannily near our thinking, and when it functions it is so little an object, that it seems to conceal its own being from us. In our analysis of the thinking of the human sciences, however, we came so close to this universal mystery of language that is prior to everything else, that we can entrust ourselves to what we are investigating to guide us safely in the quest. In other words we are endeavouring to approach the mystery of language from the conversation that we ourselves are. (p. 378)

Gadamer goes on to suggest that understanding that originates in the dialogical and that follows the logic of question and answer must take the form of language. His claim here is not that an understanding is developed and then subsequently put into words. Rather for Gadamer (1960/2003), language is the way understanding occurs, "it is the coming-intolanguage of the thing itself" (p. 378). He looks to demonstrate the linguisticality of dialogue as the fundamental basis for hermeneutics. Gadamer (1960/2003) accepts as his beginning point that "language is the universal medium in which understanding occurs. Understanding occurs in interpreting" (p. 389). ${ }^{19}$ Having established that all understanding involved interpretation, Gadamer (1960/2003) goes on to suggest that:

Like conversation, interpretation is a circle closed by the dialectic of question and answer. It is a genuine historical life comportment achieved through the medium of language...the linguisticality of understanding is the concretion of historically effected consciousness." (p. 389)

Therefore, reaching back to his earlier elaboration of the essential structures of Wirkungsgeschichtliches Bewußtsein, ${ }^{20}$ Gadamer (1960/2003) now suggests that these structures have their being within the medium of language and therefore proposes an “enigmatic intimacy" (p. 389) between thinking and speaking, and between language and thought.

\footnotetext{
${ }^{19}$ At this point Gadamer (1960/2003) recalls his earlier statement that "All understanding is interpretation and all interpretation takes place in the medium of a language that allows the object to come into words and yet is at the same time the interpreter's own language" (p. 389).

${ }^{20}$ Returning to his earlier consideration of tradition as something handed down, Gadamer (1960/2003) suggests that linguistic tradition itself "is tradition in the proper sense of the word - i.e., something handed down" (p. 389).
} 
Fundamental to Gadamer's (1960/2003) ability to elaborate the "indissoluble unity of thought and language as we encounter it in the hermeneutic phenomenon...the intimate unity of word and subject matter" (p. 403), is a requirement that he reject any conceptualisation of language in terms of instrumentalism. In so doing, Gadamer (1960/2003) lays down a challenge to any theory that does not accept this intimate unity of word and subject matter.

Language is not a mere tool we use, something we construct in order to communicate and differentiate. Both these interpretations of language start from the existence and instrumentality of words, and regard the subject matter as something we know about previously from an independent source. Thus they start too late. (p. 404)

Within the third section of Truth and Method, in a section titled 'Language and Logos', Gadamer undertakes a critique of language as seen through the history of Western thought and asserts his rejections of naïve or instrumental accounts of language. He highlights that the expression for 'word' in Greek also means 'name', most notably a 'proper name'. In following this line of thinking, Gadamer (1960/2003) suggests that it was these Greek beginnings - more specifically Plato's Cratylus ${ }^{21}$ - that conceptualised language in terms of a mere tool. Gadamer believes it was this manoeuvre that sealed the whole of Western thought on language, or rather, prevented Western thought from embracing an understanding of language that extends beyond the function of merely naming, toward an understanding of language as something that is anterior to thought itself. For Gadamer (1960/2003), approaches that account for language simply as a series of signs that unambiguously point to or designate a thing, take language to be something "wholly detached from the being of what is under consideration; taken to be an instrument of subjectivity" (pp. 416-417). In such views, language and words merely point to thought and thinking that has already occurred as an exercise of simple reflection. Gadamer, however, is not satisfied with such a conceptualisation of language on the basis of what he believes to be the intimacy of thought and language. Gadamer (1960/2003) goes on to suggest that "a word is not a sign coordinated to the thing ex-post facto" (p. 417), rather within and through words the thing comes to understanding. The following passage further exemplifies Gadamer's (1960/2003) account of language.

\footnotetext{
${ }^{21}$ Gadamer (1960/2003) states "the critique of the correctness of names in the Cratylus is the first step towards modern instrumental theory of language and the ideal of sign system of reason. Wedged in between image and sign, the being of language could only be reduced to the level of pure sign" (p. 418).
} 
Language and thinking about things are so bound together that it is an abstraction to conceive of the system of truths as a pregiven system of possibilities of being for which the signifying subject selects corresponding signs. A word is not a sign that one selects, nor is it a sign that one makes or gives to another; it is not an existent thing that one picks up and gives an ideality of meaning in order to make another being visible through it... rather, the ideality of the meaning lies in the word itself. It is meaningful already. But this does not imply, on the other hand, that the word precedes all experience and simply advenes to an experience in an external way by subjecting itself to it. (p. 417)

Thus, for Gadamer (1960/2003), "the interpreter does not use words and concepts like a craftsman who picks up his tools and then puts them away" (p. 403). Instead "language is the universal medium in which understanding occurs" (p. 389). ${ }^{22}$ In suggesting language is a medium of being human, Gadamer culminates his critique of instrumentalist conceptions of language and reaches toward a disclosure of language and thought as inextricable.

Gadamer (1960/2003) also recalls the work of Humboldt who he believes was able to establish that "a language-view is a worldview" (p. 442). At the core of this position is a view that to increase our use of language is to increase what can come to understanding of the world. Humboldt here opened up to possibility the idea that the human world is not first without language, and then emerges into language. Rather he suggests that language was human from the very beginning. Capitalising upon this opening provided by Humboldt, Gadamer (1960/2003) suggests:

Language is not just one of man's [sic] possessions in the world; rather, on it depends the fact that man has a world at all...not only is the world, world only insofar as it comes into language, but language, too, has its real being only in the fact that the world is presented in it. Thus, that language is originally human means at the same time that man's being-in-the-world is primordially linguistic. (p. 443)

Gadamer's aim in asserting that human experience of the world is fundamentally linguistic is to reach towards an assertion where humans have their very being within language. This notion of language as the medium of being human, allowed Gadamer to raise, to its highest

\footnotetext{
${ }^{22}$ Gadamer (1960/2003) opens the third section of Truth and Method with a quote from Schleiermacher - "Everything presupposed in hermeneutics is but language" (p. 381), a statement that aptly establishes the tone for the remainder of the chapter.
} 
height, his consideration of an original belonging. This is a belonging of each person to other people, and to the world. In so doing, he ultimately returns to the subject matter of the very argument with which he began Truth and Method with and overcomes the alienation of the individual from his or her experience. ${ }^{23}$

\section{The Nature of the Medium of Language}

Concerned to avoid a charge of subscribing to relativism, Gadamer (1960/2003) uses the argument of the Christian incarnation to emphasise the inextricability of word and subject matter, word and thinking and to disclose more completely his view of the nature of language. The Gospel of John 1:1-2 (NRSV) asserts, "In the beginning was the Word, and the Word was with God and the Word was God". According to the Incarnation, God the Father reveals himself as God the Son in human form. And in so doing, the Word is retained unchanged. God the Father does not turn himself into God the Son, rather, through the act of the Word becoming flesh as God the Son, the Word is retained. Thus, also in speaking, nothing is exteriorised that is somehow hidden or completely different as an 'inner', but rather is the 'inner' revealing itself.

Following this argument, then, thought (as inner) is not something different from speech (as exteriorisation). ${ }^{24}$ Instead, for Gadamer (1960/2003), "the mental word is just as consubstantial with thought as is God the Son with God the Father" (p. 421). Speaking is therefore not a process of reflection upon something internal that transforms it into something else, rather it is the word as the thing itself. This means that the inner word or thought expresses the understanding that a person has, not as a reflective viewing of it, but rather as a drawing out of that understanding through an inner dialogue. So much so that, for Gadamer (1960/2003), “All thought is speaking to oneself” (p. 422). This unity of thinking and speaking therefore implies for Gadamer (1960/2003) that the inner mental word is not formed via a reflective act, but that the words that a person speaks to him or her self are in fact the things that he or she thinks, given that "the word does not emerge in a sphere of the mind that is still free of thought" (p. 426). The following passage from Gadamer (1960/2003) is explanatory of this inextricability of word and thought.

\footnotetext{
${ }^{23}$ In a collection of essays titled Philosophical Hermeneutics, Gadamer (1977) elaborates further the indissolubility of the medium of language and the business of being human. The following succinctly captures this inextricability: "Hence language is the real medium of human being, if we only see it in the realm that it alone fills out, the realm of human being-together, the realm of common understanding and ever-replenished common agreement - a realm as indispensable to human life as the air we breath" (p. 68).

${ }^{24}$ Gadamer (1960/2003) suggests that "language is the medium in which substantive understanding and agreement takes place between two people...the language in which it is conducted bears its own truth within it-i.e., that it allows something to 'emerge' which henceforth exists” (pp. 384, 383).
} 
Language and thinking about things are so bound together that it is an abstraction to conceive of the system of truths as a pregiven system of possibilities of being for which the signifying subject selects corresponding signs. A word is not a sign that one selects, nor is it a sign that one makes or gives to another; it is not an existent thing that one picks up and gives an ideality of meaning in order to make another being visible through it...the word does not infer the mind, rather the thing intended, such that the unity of thought and word is so close that the word does not occupy a second place in the mind...rather, the word is that in which knowledge is consummated. (pp. $417,426)$

This indissolubility of mind and language suggests for Gadamer (1960/2003) that there is no possibility that a person can begin to "see a linguistic world from above... for there is no point of view outside the experience of the world in language from which it could become an object" (p. 452). Thus the person that Gadamer has in mind does not come to an experience and then name it. Instead, the very possibility of having an experience at all is born of language. The following passage from Gadamer (1960/2003) is illustrative of this.

Experience is not wordless to begin with, subsequently becoming an object of reflection by being named, by being subsumed under the universality of the word. Rather, experience of itself seeks and finds words that express it. We seek the right word - i.e., the word that really belongs to the thing - so that in it the thing comes into language. Even if we keep in mind that this does not imply any simple copying, the word still belongs to the thing insofar as a word is not a sign coordinated to the thing ex post facto. (p. 417)

For Gadamer, then, language is the medium within and through which experience transpires and allows a thing to be understood - to come into meaning. In seeking to apprehend further the fundamental nature of what language is, Gadamer recalls the words of St. Thomas who conceives of the word as light. In the same way that light that shines upon something and makes it visible, the word makes things appear. The concept of colour provides a most suitable example. In the absence of the word, a colour cannot become itself. That is, a thing cannot be red until the thing itself comes into word as red. Thus, in and through words - as language - the thing appears as itself for the person whose words - as light - illuminate the thing and therefore allow it to be understood.

Importantly, Gadamer's conceptualisation of language as being analogous to light, is not to be considered a slippage into an instrumentalism, whereby the word becomes little 
more than the scanning spotlight that seeks the objects intended. Rather, the idea that the word is consistent with the notion of light is a reaffirmation that language, thought and understanding are indissoluble. In fact, for Gadamer (1960/2003), in the absence of language a person cannot begin to see the thing that is to be understood, let alone bring it to intelligibility. ${ }^{25}$ The medium of language is therefore not an obstacle to be overcome in understanding, instead it is the very possibility of being itself. The following passage is exemplary of Gadamer's (1960/2003) position.

This is of fundamental importance, for it makes the expression "world in itself" problematical. The criterion for the continuing expansion of our own picture is not given by a "world in itself" that lies beyond all language.

Rather, the infinite perfectibility of the human experience of the world means that, what ever language we use, we never succeed in seeing anything but an ever more extended aspect, a "view" of the world. Those views of the world are not relative in the sense that one could oppose them to the "world in itself," as if the right view from some possible position outside the human, linguistic world could discover it in its being in itself...this is part of the meaning in which every human, linguistically constituted view of the world lives. In every worldview the existence of the world-in-itself is intended. It is the whole to which linguistically schematized experience refers (p. 447).

For Gadamer (1960/2003), then, the multiplicity of these worldviews does not involve a mere relativisation of the world, "rather what the world is is not different from the views in which it presents itself' (p. 447). Here this 'presenting itself' is not an acceptance of the world as a metaphysical entity that is consciously deliberate in its choice of response to a specific question. Instead it is an acceptance that the world exists independently of human understanding of it, and only comes to be meaningfully understood in and through language.

On this, Gadamer recalls his earlier consideration of the coalescence between the person and the world as a dialogue that allows the essential being of that which is other to come to intelligibility. The inexorability of this dialogue for Gadamer (1960/2003) can be seen in his statement that "not only is the world world only in so far as it comes into

\footnotetext{
${ }^{25}$ Wachterhauser (2002) also elaborates the essential nature of language in terms of providing a light or a standpoint that enables a thing to be understood meaningfully; "One could say that the reality of the world's intelligibility is not dependant on our standpoint, but the way in which we are able to access that intelligibility is always dependant on such standpoints to some degree... in principle, what a 'standpoint' is capable of delivering to us is a 'piece' of the world, a 'view' of the world, a 'perspective' on the world. In principle, a standpoint renders the world accessible, not inaccessible" (p. 74).
} 
language, but language, too, has its real being only in the fact that the world is presented in it" (p. 443). Therefore, for Gadamer, all understanding is foregrounded upon the backdrop of a shared world, perceived through the lens of a unique linguistic 'worldview'.

\section{The Disclosure of the World in Language}

One could be forgiven for seeking to challenge Gadamer's thesis concerning the inextricability of language and understanding on the basis that it does not hold for all things to be understood. Pieces of art or works of music are but two examples that surely do not require the use of language. However, recalling Gadamer's earlier discussion about the truthvalue of art, we will recognise that these forms of art are not devoid of the possibility of providing understanding and as such must be capable of coming into language. In other words, the way in which a person brings the art to life is not different from the way in which it comes to language for that person, not albeit as a performative act, but rather as an understanding itself. Grondin (2003) captures the essence of Gadamer's position on this possibility of coming to language, stating that "the unsayable, the unnameable everything that is outside language, from the time it is evoked or invoked, even if it has to remain voiceless is at least capable of being said" (p. 128). It is from this position that Gadamer (1960/2003) reaches forward with arguably his most substantial contribution to the hermeneutic movement.

We can now see that this activity of the thing itself, the coming into language of meaning, points to a universal ontological structure, namely to the basic nature of everything toward which understanding can be directed. Being that can be understood is language. (p. 474 emphasis in original)

Grondin (2003) argues that Gadamer's thesis becomes clearer when worded in the negative "the being which cannot be understood is that which is not language" (p. 128). Thus, what cannot become language cannot be understood. To bring a thing into language is to bring that thing to understanding. It is this capacity for being said of the world, and therefore as a coming to understanding, that opens up the universality of Gadamer's hermeneutics to a 'wanting-to-say'. That is, if the fundamental comportment of being is understanding, and that language discloses the thing to be understood itself, then the wanting to bring the world to language and therefore understanding is the universal inclination for each of us. A look at Gadamer's notion of the Mot Juste is a valuable elaboration of the nature of this inclination toward bringing the world to understanding.

In an essay titled 'The Limits of Language' (1985), Gadamer outlines what he describes as the unsatisfying search for the mot juste or the perfectly appropriate word or 
phrase for a situation. Here Gadamer (1985), cited in Grondin $(2003)^{26}$, suggests that the constant search for the mot juste is the embodiment of human life itself and the genuine essence of language.

Knowledge lives in everybody who speaks when he looks for the mot juste to know the word which can reach the other - but it always knows at the same time that it has not completely found it. The wanting-to-say, the intention, always goes above, or slantwise to, what can be truly encircled by a language or in the words which go to rejoin the other. (p. 129)

The unsatisfying aspect of the search - characteristic of mot juste - is the person's awareness that knowledge of the thing to be understood can never be perfect, it can never be complete. Instead coming to know the thing better involves a journey that continues in time from birth to death.

\section{The Speculative Nature of Language}

Gadamer is keen to overcome any challenge to the ontological presence of language as merely a surreptitious instrumentalism, whereby language is simply a means for coming closer and closer to knowledge of the thing itself. Forestalling this argument, he considers the speculative nature of language as a productive path to pursue. Gadamer (1960/2003) suggests that language is the medium of being human, a medium through which the thinking being unfolds "what consistently follows from the subject matter itself" (p. 464). This unfolding for Gadamer takes the form of a dialogue. Here Gadamer (1960/2003) suggests a "dialectic of the word, which accords to every word an inner dimension of multiplication" (p. 458), and which allows the whole of a person's world-view to appear. Thus each word carries within it the unsaid, or as Gadamer (1960/2003) put it, "allows an infinity of meaning to be represented within it in a finite way" (p. 465). ${ }^{27}$ The following passage from Gadamer $(1960 / 2003)$ illuminates this point further.

Language itself has something speculative about it in a quite different sense as the realization of meaning, as the event of speech, of mediation, of coming

\footnotetext{
${ }^{26}$ This original quote appeared in Gadamer 's (1985) Gesammelte Werke Vol 1-10, Tubingen, Mohr. It represents a work that is not available in an English translation.

${ }^{27}$ The speculative nature of language provides a further avenue to apprehend the way in which an individual always belongs to the world by apprehending that the experience of language is more than merely something that humans use. Here Gadamer raises the notion of Hören, the German word for hearing. In hearing one is reached by the whole of that which can be understood. The notion of hearing therefore provides Gadamer a further avenue to disclose the way that a person belongs to the world through language. For a more thoroughgoing consideration of this notion see Truth and Method (1960/2003, p. 462).
} 
to an understanding. Such a realisation is speculative in that the finite possibilities of the word are oriented toward the sense intended as toward the infinite. (p. 469)

Gadamer is clear that language is finite while allowing an infinity of meaning to be represented. It is this capacity of language to carry meaning that goes well beyond simply that which is said that enables Gadamer to overcome a conceptualisation of his work as simply another representation of a linguistic instrumentalism. In fact, when language is not considered in terms of its speculative capacity - where meaning is reduced purely to that which is said - a distortion is inevitable. Gadamer (1960/2003) uses the example of a statement to highlight this.

Anyone who has experienced an interrogation - even if only as a witness knows what it is to make a statement and how little it is a statement of what one means...But meaning thus reduced to what is stated is always distorted meaning. To say what one means, on the other hand - to make oneself understood - means to hold what is said together with an infinity of what is not said in one unified meaning and to ensure that it is understood in this way. (p. 469)

Thus the speculative conceptualisation of language operates to emphasise that language is not a tool for merely reflecting something that is given. Instead, for Gadamer (1960/2003), to situate language as a medium of being is to allow "the coming into language of the totality of meaning" (p. 474). Language does not therefore represent a vehicle for the captivity of human understanding, rather it is the very condition of human understanding itself.

For Gadamer, the speculative nature of language means that everything that comes to understanding in language is said, but can always be said in a different or perhaps a better way. Recalling Hegel, Gadamer (1960/2003) notes that the speculative-person does not abandon him or herself to dogmatism, but rather, recognises that "the 'in-itself' is a 'for-me"” (p. 466). Here, then, understanding is the way in which the thing that is to be understood comes to understanding for that individual person. Such a conceptualisation of understanding returns all the more to the development of a person's Wirkungsgeschichtliches Bewußtsein as a self-understanding and an openness to further understanding. Recognising that an understanding is indeed an understanding as it is 'for me' epitomises this openness. In addition, the speculative conceptualisation of language is a recognition that it is always possible to understand one's Self differently, moving from a present understanding - borne of a person's historicity - to another different understanding. By seeing language as the medium 
of this essential mediation between past and present, Gadamer (1960/2003) establishes that the finite historicity with which we illuminate a situation is that which we apprehend in learning to speak. This position is outlined further through this passage.

Man's [sic] relation to the world is absolutely and fundamentally verbal in nature, and hence intelligible. Thus hermeneutics is, as we have seen, a universal aspect of philosophy, and not just the methodological basis of the so called human sciences. (pp. 475-476)

The final section of Truth and Method, entitled 'Language as the Horizon of a Hermeneutic Ontology', draws Gadamer's position to its conclusion. Here Gadamer (1960/2003) makes clear that for him language is the horizon of being human. It is the vantage point from which a thing can become intelligible as well as that beyond which we are unable to see. ${ }^{28}$ The ontological presence of our horizon is such that we are unable to rise above it to a different position in order to see the whole of our being. However, we are always able to expand our horizon by finding other words that allow us to apprehend the world in different ways, a world to which at every moment each of us belongs through the medium of language.

\section{Chapter Summary}

This chapter has provided a consideration of Gadamer's (1960/2003) philosophical position as a means of locating language as the inextricable medium of being human. To this end, Gadamer's hermeneutic position ultimately suggests that 'Being that can be understood is language' (p. 474). Gadamer was able to show that each person is always connected to the world by way of his or her Wirkungsgeschichtliches Bewußtsein. The fundamental comportment of each person toward understanding means that one reaches forward from the position of his or her own horizon by way of an initial prejudice. Through the movement of dialogue that follows the structure of question and answer, each person comes to a new or different understanding of the thing that is to be understood. For Gadamer, understanding is a fusion of horizons. Here the horizon of the past - the person's Wirkungsgeschichtliches Bewußtsein - fuses or coalesces with the horizon of the present, or the thing to be understood in understanding. For Gadamer, it is language that makes the fusion of horizons possible. Importantly, this inextricability of language from understanding as the comportment of being does not represent a dogmatic view of the possibilities of understanding that are available to

\footnotetext{
${ }^{28}$ The use of the word 'see' here is most apt given Gadamer's use of the notion of light in the final pages of Truth and Method. More specifically, he recalls the arguments concerning 'the beautiful' and the notion of light, and by making some clear parallels between light and word, and suggests that without light nothing that is beautiful can appear and therefore nothing can be beautiful.
} 
the person. Rather, it embodies the way in which language is the very condition of understanding itself. Thus, in conceptualising language in terms of its speculative nature where words carry meaning that goes well beyond simply what is said - the comportment of the person that Gadamer (1960/2003) envisages is always toward finding the Mot Juste - the most perfect word or phrase - for bringing the world to understanding and the carriage of that understanding to another person.

\section{Section Summary}

As well as providing a consideration of the position Gadamer established, this chapter draws to a close the first section of the thesis and provides a suitable juncture for reflection upon the journey thus far, as well as the task ahead. It was suggested that as a result of the perpetuation of a naïve assumption about the nature of language and meaning, qualitative research has contributed less to the understanding of the individual person's qualia than is considered to be possible. A critique of the processes of representation that are common within contemporary approaches to qualitative research underscored a progressive movement away from the participants' dialogue itself, and therefore away from the representation of the experiences of real people and the real lives encountered.

A review of the Criteria Debate highlighted that the representation of 'a real' would appear to be of limited concern to the discipline of qualitative research, problematising further the legitimacy of the representations of real people and real lives that are produced. It was suggested that this progressive movement away from 'a real' was incapable of providing a sufficiently deep level of consideration of the individual participant. In other words, the search for generality that lay at the foundation of the pervasive subscription to thematic representation within qualitative research leads to a neglect of bringing to understandings the deeply nuanced aspects of human experience.

The neglect of deep understanding of the human experience leads this thesis to suggest that the focused consideration of the individual person is the way forward for qualitative research. It is suggested that a focus upon the individual person at the level of his or her qualia, will provide an avenue for overcoming not only the representational assumptions that permeate qualitative research but will address the dearth of understanding at the level of the individual person in qualitative research. It was on the basis of this that the thesis turned toward Personal Construct Psychology as embodying - in principle - the idiographic position with which this thesis holds most hope. 
Despite the high level of abstraction with which Kelly (1955) unpacks a consideration of the individual person, he located the construct as a fundamentally ontological structure that has its being outside of language. In so doing, he opened up to skepticism the theoretical tenability of his approach and the suitability of his position for informing the development of a reflexive approach for qualitative research. A consideration of the broader implications of the way in which Kelly dealt poorly with language for Personal Construct Psychology was undertaken. This undertaking highlighted that if the theoretical principles espoused by Kelly (1955) are to be considered to be theoretically reflexive and therefore consistent with 'a realism', an elaboration in the direction of language is required. Thus, it was toward the hermeneutic work of Gadamer that this thesis then turned.

The philosophical hermeneutic position elaborated by Gadamer, situating language as the medium of being, provides a productive foundation for re-envisioning many of the principles embodied by Personal Construct Psychology. This re-envisioning will in turn provide a theoretical position that is able to better inform a deeper and more sophisticated qualitative research. Thus, it is in the direction of this re-envisioning - the focus of the second section of this thesis - that this thesis now turns. 


\section{CHAPTER 6}

\section{THE IDIOGRAPHIC WITHIN A LANGUAGED WORLD}

The three previous chapters provided the groundwork for a theoretical synergy of Personal Construct Psychology and Hermeneutic Phenomenology in this chapter. Accepting that the business of being human involves an inextricability of a person's mental activities and the medium of language, this chapter elaborates a position termed Hermeneutic Constructivism. In elaborating the Hermeneutic Constructivist position, the framework for theoretical elaboration that is familiar to Kelly's work is adopted. Therefore this chapter will set down Hermeneutic Constructivism and elaborate this position through a fundamental postulate and subsequent elaborative corollaries. The following chapter will explore the more abstract ideas that arise out of the Hermeneutic Constructivist position proposed here.

\section{Building a Theory}

A theoretical elaboration is always founded upon a series of pre-conceived assumptions. The role of this chapter is to set down those assumptions. To facilitate this the model that Kelly (1955) adopted in expounding The Psychology of Personal Constructs will be adopted here. As outlined previously, Kelly (1955) established an overarching theoretical position - Constructive Alternativism - and sought to elucidate this theoretical assumption through a Fundamental Postulate and a series of corollaries that amplify this position. For each of these corollaries he provided a descriptive elaboration that was designed to unpack the theoretical assumptions that inform that position. Given that it is Kelly's (1955) model that is adopted for exploring the vicissitudes of the theoretical position being developed here, it is useful to be more specific about what he meant by the notion of a Fundamental Postulate.

For Kelly (1955) the most basic statement of a theoretical position is the Fundamental Postulate. This is a statement that is basic to all the theoretical elements that it supports and provides a tentative proposal from which to begin. Kelly (1955) believes that it is easy for us to pose questions concerning the postulate, but asks that we recognise that these questions are in fact arising from a different set of assumptions to those embodied by the postulate itself. The following passage from Kelly (1955) is elaborative of this, "the moment we do question the truth of a statement proposed as a postulate, that statement is no longer a postulate in our subsequent discourse. A statement, therefore, is a postulate only if we accord it that status" 
(p. 47). At the core of his suggestion is a request that a reader does not seek to move toward a different theoretical position until the fundamental postulate itself and its supporting propositions or corollaries are elaborated. With this in mind, a turn is made toward the elaboration of a series of theoretical assumptions that are fundamental to the proposed reenvisioning of Kelly's (1955) Personal Construct Psychology from the foundation provided by Gadamer's (1960/2003) Hermeneutic Phenomenology. This is a position termed Hermeneutic Constructivism.

\section{Hermeneutic Constructivism}

What is it about qualitative research that makes it qualitative? As outlined in the introduction to this thesis, a simple dissection of the word qualitative highlights that the focus most certainly must be concerned with or have a propensity for an exploration of qualia. Qualia have been described as the subjective qualities of conscious experience, or in another way the 'What is it Like' character of mental states (Wright, 2008). Thinkers operating within the field of Philosophy of Mind research agree that although each individual person has conscious experience - or qualia - their interpersonal comparison is systematically impossible. Qualia are unique to the experiencer. ${ }^{1}$ Given this, a discordance is considered to exist between qualia as the currency of qualitative research, and established practices within qualitative research as outlined in earlier chapters. ${ }^{2}$ This discordance brings to the fore two key notions from which the movement of the theoretical re-envisioning being proposed here originates: (i) that less is known about the individual people included in qualitative research because of the assumptions made about them, and (ii) that language is the essential medium

\footnotetext{
${ }^{1}$ It is important to be clear that by suggesting that qualia are inherently unique is not to subscribe to 'private language' as critiqued by Wittgenstein (1953/1968). Instead it is to recall the speculative nature of language as outlined by Gadamer (1960/2003), where language carries meaning that goes well beyond merely that which is said. The finite possibilities of the words uttered carry infinite possibilities of meaning. Thus, given that for this thesis the notion of being is inextricable from language, the fundamentally indeterminate nature of language makes the possibility of elaborating the meaning of another person's entire historicity or prejudices unimaginable.

${ }^{2}$ For a thoroughgoing review of these practices - common to qualitative research - please see Chapter Two of this thesis. The following provides a brief sketch of the core elements of the argument outlined within that chapter. It is assumed that a participant or participants can bring to language their experiences of and with phenomena - their qualia - in such a way that the researcher is able to gain access to them. So far so good. However, more than bringing those qualia to light, the researcher is supposedly able to access the hidden meaning that is behind those words. In other words, the participant brings to language his or her qualia and then the researcher transcends the words uttered and somehow accesses the original thought of the participant. Here the researcher is then able to bring to light what it was that the participant actually meant by their statements. Subsequently, the researcher is considered able to represent the qualia of his or her participant(s) more fully than the participant(s) are able to articulate them themselves. Thus the researcher is now able to make comparisons between entities that are ultimately and systematically incomparable. For this thesis, however, the subscription to a naïve or transparency view of language that is inherent and also integral to this so-called movement of understanding raises doubts about the degree with which such research is genuinely qualitative in nature.
} 
of the business of being human. On the basis of these assumptions, this thesis proposes that a Hermeneutic Constructivist position will provide a more useful way of catching a deeper and more sophisticated glimpse of the individual going the business of being human for qualitative research. A look now at the fundamental assumptions that inform the Hermeneutic Constructivist position is essential.

\section{The World}

Standing in need of elaboration in the setting-down of Hermeneutic Constructivism is the world and more specifically the type of world envisaged. Experience is always experience of something; in fact, it is always experience of the world. Although the world does and perhaps one day will exist independently of human affairs, it is a continuously happening entity. ${ }^{3}$ In other words, at any one moment in time there are innumerable happenings throughout the world. Each person will never come to appreciate all of these, for the fact of the matter is we cannot, at any single moment in time, direct our gaze toward each and every happening. Instead, only those aspects that we can appreciate are those towards which we direct our understanding processes. Those aspects of the world that we direct our understanding processes toward are here termed events.

Hermeneutic Constructivism takes the view that the fundamental comportment or attitude towards the world that each person adopts is one of understanding. In other words, each of us seeks or is rather compelled to understand the world in order to go about within the world. Understanding, however, is not an understanding in terms of the things in themselves as a perfection of understanding. Instead this understanding is an understanding of the thing of itself, an understanding that is sufficient in order to go on with being human, to go on with the world. Therefore, if the comportment of being is toward understanding, and those aspects of the world that we reach toward in understanding are events, then being itself is an active movement on the part of the person that can be embodied in terms of a being situated within a stream of events.

\section{Person the Languager}

Taking the view that language is the medium of being human, Hermeneutic Constructivism establishes that language is more than merely an instrument for working on

\footnotetext{
${ }^{3}$ Of course these assumptions are not foreign to the work of both Kelly and Gadamer, who agree on the presence of a world independent of human constructions of it. Gadamer (1960/2003) states, "no one doubts that the world can exist without man and perhaps will do so" (p. 447). Kelly (1955) is even more explicit about his convictions: "We presume that the universe is really existing and that man is gradually coming to understand it. By taking this position we attempt to make clear from the outset that it is a real world we shall be talking about, not a world composed solely of the flitting shadows of people's thoughts" (p. 6).
} 
the world. ${ }^{4}$ In taking this view, the person that this thesis envisages does not have an experience and then name it. Instead it is always within and through language that we come to understand and experience the world. It is therefore language that nourishes and sustains our ongoing movement of being human. From this foundation, it is clear that the person with whom Hermeneutic Constructivism is concerned is not 'Person the Bystander', or 'Person the Pointer to Things', but rather 'Person the Languager'.

This is no trivial label. Instead it represents the fundamental assumption that informs Hermeneutic Constructivism. In fact, from here on, each individual person is thought of in terms of 'Person the Languager', a person who through and within the medium of language comes to experience and understand the world in such a way that there can be no separation between the world and his or her experience of it. So much is this the case that the psychological processes of 'You the Languager' are indissoluble from the medium of language. This means that having an experience is itself not different from the way in which it can be brought to language, making 'You the Languager's' relation to the world primordially linguistic and hence intelligible. Further recourse will be made in the following chapter to the theoretical intricacies that underpin the notion of 'Person the Languager'. In order to go on with the setting down of the Hermeneutic Constructivist position, a brief sketch of the way each person engages in a dialogue with the world is necessary.

A dialogue between two people is easy to conceive of. However the world itself cannot speak and therefore engaging in a dialogue with it is less straightforward to comprehend. Here Gadamer's (1960/2003) consideration of $I$ - Thou relationships is recalled. ${ }^{5}$ For Gadamer (1960/2003) the $I$ encompasses all that is the Self. It is the culmination of our historicity as that something that we have made of things within the world in previous ventures of understanding. This $I$ therefore represents all that we bring with us to an event of understanding, and embodies the possibilities that we already have of understanding. A Thou on the other hand is all that is other. Not in terms of objects themselves, but rather in terms of the way in which things in the world are able to have a relation with the $I$. That is, the $I$ makes the thing to be understood come to understanding in such a way that it can relate to the $I$ through a dialogue as a Thou. In order for this to occur the $I$ asks questions of the thing to be understood. In so doing the thing is then able to relate to the $I$. Here, then, a thing becomes a Thou and relates to the $I$ by providing answers to the questions asked of it. The complexity of this relationship lends itself to an exemplar.

\footnotetext{
${ }^{4}$ This view is well founded within Wittgenstein's (1953/1968) own 'linguistic turn' and most clearly within Gadamer's (1960/2003) hermeneutic elaboration. These positions have been explored within Chapter Four and Chapter Five of this thesis.

${ }^{5}$ For a more thoroughgoing exploration of Gadamer's elucidation of I-Thou refer back to Chapter Five.
} 
The first time someone is in love, he or she cannot understand that something that is clearly happening to them. ${ }^{6}$ The constantly intrusive thoughts about someone else and the overwhelmingly positive emotion are outside of anything experienced before and therefore cannot easily be understood. The recognition of this something as an overwhelmingly positive emotion is a function of the $I$. Initially it is conceivable that an understanding of these sensations, as an overwhelmingly positive emotion, is all that the person has available to him or her in order to make sense of what is going on in the here and now. However, in the excitement of coming to know better what it is that is going on, that person begins to ask questions. These questions originate from the $I$ and are directed at the emotion. Therefore, the questions make the emotion become a Thou that in turn provides answers to these questions.

After a to and fro dialogue between $I$ and the emotion as a Thou, the person may arrive at the joyful conclusion that this feeling must be love. Here the circularity of question and answer, from $I$ to Thou and back again as captured by this exemplar, is the way in which the Thou relates to the $I$ and ultimately comes to change the $I$ for future anticipations. In other words, the understanding of the emotion as an initially overwhelmingly positive emotion that then comes to be understood as love, becomes a part of the $I$ in that it becomes a part of the person's repertoire of understanding in the future. Thus, in setting-down the theoretical foundation of Hermeneutic Constructivism, the term 'dialogue' relates not only to what goes on between people, but through the notion of $I-T h o u$, represents that which also goes on between the person and the world.

\section{Prejudice Systems}

This thesis takes the view that within and through language, as dialogue, people develop for themselves a series of historically mediated, anticipatory and languaged structures through which the world comes to be meaningfully understood. These structures are referred to as prejudices. When projected towards an aspect of the world or as an event of understanding, a person may find that his or her prejudice(s) is less able to adequately bring that event to satisfactory understanding. ${ }^{7}$ Although a prejudice may be found to provide an

\footnotetext{
${ }^{6}$ Love is of course a highly charged and contested term within the psychological and philosophical literature. However, here love is used as an example of an emotion that at first a person is unable to apprehend in understanding and yet over time and through continued dialogical engagement with the Self, comes to be understood as such. Moreover, the example of love provides an opportunity to explore the way in which many of the Corollaries of Hermeneutic Constructivism 'play out' in a foreseeably common context.

${ }^{7}$ It is important to apprehend that this thesis is not subscribing here to a simple or naïve realism where each prejudice merely corresponds to a piece of the world. On the contrary, this is recognition that the possibilities of human understanding are finite. We project a prejudice toward the world in
} 
inadequate understanding and therefore stand in need of development, a prejudice is itself an inextricable precondition of understanding. That is, all understanding originates entirely in the light provided by a person's prejudices.

As elucidated by Gadamer (1960/2003) and outlined in the previous chapter, the term prejudice is given to these 'conditions of understanding'. Importantly, the use of the term prejudice here is not to be confused with its common meaning as a false or irrational thought or as a prejudice against a thing. Instead it is understood in its original meaning as a judgment rendered before all available elements for consideration have been finally examined. Importantly the prejudice is not a structure of human understanding that restricts the possibilities of understanding. That is, the prejudice cannot be conceptualised in terms of a search light that simply seeks those things in the world that correspond with it. Instead, a prejudice is a temporal and yet essential precondition of understanding. It represents the initial position from which an understanding originates. It is through the dialogical movement of the I-Thou relationship outlined above that such an initial understanding is developed toward a different or better understanding.

As a structure that represents the position from which all understanding originates, the prejudice is therefore inextricable from the human comportment toward understanding. So much so that a prejudice that provides an inadequate understanding is in fact better than no prejudice at all. In other words, we cannot begin to reach toward the world in understanding independently of our prejudices. Importantly, however, no single prejudice can be developed that is capable of bringing to understanding every event that the world has to offer. It is for these reasons that each person develops a series of prejudices that are related to each other and together form his or her greater system of prejudices.

Although this thesis has not yet attempted to be explicit about the way in which the prejudice develops dialogically, enough has been said to establish that the prejudice is a representation of the world that is developed by the person, and has its being within language. Through the projection of a prejudice toward an event, a person determines the adequacy of that prejudice and dialogically adjusts that prejudice in the direction of the possibilities opened up through questioning. Thus, at its core, the business of being human refers to the projection of a prejudice towards the world - as an event - so as to satisfactorily understand

understanding and through a dialogue make the world respond - as a Thou - to the questions asked of it. However, the $I$ can always pose different questions of the world and receive different answers. Thus, it is our prejudices that provide the possibility of understanding and the beginning point for a dialogue. Importantly, this is not a simple relativism, because it is the world itself that is being made to answer the questions asked of it as a Thou. 
the world in order to go on being in the world. Inherent within this conceptualisation of the prejudice is a inclination to find ever better ways of anticipating the world.

\section{Statement of Hermeneutic Constructivism}

It has been noted that within and through the medium of language each person develops for him or her self a series of related prejudices through which the world can come to understanding. To this extent, for Hermeneutic Constructivism, the business of being a person involves an inextricability between the mental activities of the individual person and the medium of language. This statement will have bearing upon almost everything that is to be said from hereon in. In fact, following the lead of Gadamer, this thesis takes as its starting point that language is the medium of the business of being human. Not in terms of a restriction to the possibilities of human understanding, but rather as its very condition. At the centre of this core is a needing to understand, not in terms of a human desire but rather as the fundamental comportment of being human. Here a decisive philosophical foundation is established that informs the direction taken in the re-envisioning of Personal Construct Psychology and from which the theoretical position of Hermeneutic Constructivism ${ }^{8}$ can spring forth for catching a more sophisticated glimpse of the individual person for qualitative research.

Prior to moving to the formal elaboration of Hermeneutic Constructivism, it is important to establish clearly that this thesis is not seeking to develop a psychology that underpins psychotherapeutic activity in the way Kelly did. In light of this, several of Kelly's theoretical maneuvers are superfluous to the needs of Hermeneutic Constructivism and will therefore not find a parallel consideration in this elaboration. Hence, although Kelly's (1955) work can be read between many of the lines of Hermeneutic Constructivism, there are many new elements pursued here.

\section{Formal Elaboration}

FUNDAMENTAL POSTULATE - A PERSON'S UNDERSTANDING PROCESSES ARE GUIDED BY HIS OR HER LANGUAGING OF EVENTS.

This postulate embodies the principles of Hermeneutic Constructivism noted above. Language is the foremost medium through which the proactivity of human understanding takes place. As captured by the notion of Person the Languager, language is for Hermeneutic Constructivism the condition of human understanding. To help capture what is meant by the

\footnotetext{
${ }^{8}$ See Chapter Four for a consideration of the work of Chiari and Nuzzo, who coined the term Hermeneutic Constructivism.
} 
notion of a medium, a cognitive demonstration as outlined by Wittgenstein is useful. In seeking to highlight the inseparability of mental activities from language, Wittgenstein (1968) leads us through the following exercise.

Point to a piece of paper. - And now point to its shape - now to its colour now to its number (that sounds queer). - How did you do it? - You will say that you meant a different thing each time you pointed. And if I ask how that is done, you will say you concentrated your attention on the colour, the shape etc. But I ask again: how is that done? (p. 16)

Here Wittgenstein highlights that the action of merely pointing is the same behaviour each time and therefore that the action alone cannot carry different meanings. The same problem arises when a person believes that he or she points to each of these elements mentally. In fact, in each situation the movement of pointing requires some expression of what we are meaning each time and cannot therefore occur in the absence of language. ${ }^{9}$ Wittgenstein's cognitive experiment highlights that language is the medium through which the world comes to be understood. Language is the medium of a person's understanding processes. Given that this postulate will not hold the same meaning for all that read it, and with a view to its operationalisation in the future, an elaboration of the words chosen for this postulate is valuable.

Persons - This term indicates the subject matter of focus is the understanding processes of the person and emphasises that the focus is first and foremost the individual person.

Understanding - It is suggested that understanding is the totality of what a person can bring to language about a thing that is to be understood. In other words, if we were to ask another person to tell us about something, then what he or she could bring to language on the subject matter represents what that person understands about that something. Conceived in this way, understanding is therefore that something that a person already has. One must recall that understanding here is not a perfection, but instead, that something that is required in order for that person to go on in the world. The notion of understanding as the human comportment that is implicit in this conceptualisation of understanding itself becomes clearer when considered in reverse. That is, in the absence of a sufficient understanding, the world appears

\footnotetext{
${ }^{9}$ In capitulating his position established in the Tractatus Logico-Philosophicus (1922/2005), Wittgenstein concedes that all meaning is language, such that we are unable to represent the world without language.
} 
as little more than an indefinable and overwhelmingly ambiguous array of sensory input. ${ }^{10}$ Having established that understanding is the comportment of being, the nature of this understanding can be elaborated further.

The prejudice has been described as a historically mediated structure. That is, it embodies what a person has made of time-past and now uses to project toward time-present in order to bring it to understanding. It is therefore this prejudice that is projected, from which understanding is ultimately achieved. Through the I-Thou dialogue outlined above, this initial prejudice has the possibility of evolving through the dialogue of question and answer. Although at the end of this dialogue a sufficient understanding is achieved in order for a person to go on being in the world, this understanding always has the possibility of becoming a different or better understanding than the initial prejudice that was projected.

Here the emphasis upon understanding as a self-understanding is palpable. Given that it is the $I$ that not only projects the initial prejudice but also poses questions to the thing that is to be understood, in so doing initiating and sustaining the I-Thou dialogue, it is therefore the $I$ that is ultimately developed by this $I$-Thou dialogue. In other words, if the $I$ embodies the totality of that something that we already have about a thing, and simultaneously conditions the dialogue of I-Thou, it follows that what we come to understand better or differently from the I-Thou dialogue is in fact the $I$. With this in mind the nature of the $I$ can be elucidated further. The $I$ is conditioned by our prejudices as the culmination of that something that we have made of the events of time-past. It follows, therefore, that if the prejudice is the essential condition of the $I$, then it is in fact the prejudice itself that is developed through the dialogue of I-Thou.

This notion of the $I$ is for Hermeneutic Constructivism synonymous with the Self. This is because the $I$ is the totality of our prejudices or those structures that determine our understanding processes and is therefore the foundation of our highly nuanced understandings of the world, and what makes us who we are as individual people. Thus, achieving a greater understanding of a person's prejudices is to understand that person at the level of the Self.

Processes - The use of this term within the fundamental postulate emphasises that understanding is an active process on the part of the person who wishes to understand. Given that all understanding originates from the prejudice(s) that we project towards the world, the person that Hermeneutic Constructivism concerns itself with is considered to be

\footnotetext{
${ }^{10}$ The example of Sensory Agnosia is exemplary of this. Often limited to one of the senses, a person with this condition is unable to bring an object, a person, or a smell, to understanding, and therefore to language, although his or specific sense, and memory is intact.
} 
fundamentally proactive when it comes to understanding. Moreover, by suggesting that understanding is the fundamental comportment of a person's being, it follows that it goes on from birth to death. Thus this person can be considered as being in a constant state of motion or change even if only with respect to him or her self at any given moment in time.

Guided - To be guided is to be directed or influenced regarding the course of action that is taken. Here the term emphasises that a person's understanding processes are always under the direct influence of or follow already established pathways. ${ }^{11}$ Remaining consistent with the idea that a prejudice is an anticipatory structure from which all understanding originates, it follows that these same structures exert an influence over the process of understanding. In other words, these structures guide the movement of understanding. Importantly, however, being synonymous with a person's prejudices, these guides are temporal and as such are themselves open to ongoing revision through the dialogue of understanding. Despite the inherently temporal nature of these guides they are nonetheless fundamentally ontological in nature.

Languaging - Hermeneutic Constructivism takes the view that language is the medium through which the world comes to understanding. Here, then, there is no separation envisaged between the thing to be understood and the way in which the person brings it into language. This is not a coming into language as an act of reflection, where the thing is thought about and then brought into language. Instead the way a thing is ultimately understood is not different from the way it comes to language; it is coming into language as the thing itself. ${ }^{12}$ Thus, language is the medium of the fundamental comportment of being toward understanding. ${ }^{13}$ The term 'languaging' is given to the dialogical movement of this comportment of bringing the world to understanding. However, prior to a further unpacking of languaging itself, a look at the way that meaning is differentiated from understanding is useful.

\footnotetext{
${ }^{11}$ Kelly (1955) adopted the term "Channelized" (p. 49) to apprehend the way in which our psychological processes are guided. However this term is less commensurate with the fluidity of the understanding processes that are anticipated by the Hermeneutic Constructivist position. It is for this reason that the word guided is adopted here.

${ }^{12}$ Here we are reminded of Gadamer's (1960/2003) use of the Christian Incarnation as a vehicle for accentuating the consubstantial nature of word and thought. That is, in speaking, nothing is exteriorised that is hidden, or completely different as 'inner', but rather is the 'inner' revealing itself. In other words, thought (as inner) is not something different from speech (as exteriorisation), instead "the mental word is just as consubstantial with thought as is God the Son with God the Father" (p. 421). Therefore speaking is not a process of reflecting upon something internal and transforming it into something else, rather it is the word as the thing itself. For a more complete discussion of this, please refer back to Chapter Five The Nature of The Medium of Language.

${ }^{13}$ Gadamer (1960/2003) captures this nexus between language and that which is to be understood as follows: "it is the coming into language of the thing itself" (p. 378).
} 
Understanding has already been described as all that a person can bring to language about a thing to be understood. It represents that something that has been previously made of an event of understanding. As such it also represents the initial possibilities that are available to a person from which to begin a dialogue toward understanding in the future. The notion of meaning, however, represents what it is that goes on in making something of the world. That is, meaning is the term given to the dialogical to and fro movement of making a determination of the appropriateness of an understanding. This determination is not the seeking of a correspondence between a prejudice and the thing to be understood. Instead this determination is a consideration of the degree to which an understanding is satisfactory or sufficient in order for the person to go on with the world. Meaning is therefore the dialogue of I-Thou itself. It is the working out of how well an understanding that a person already has is able to satisfactorily bring to understanding a specific event toward which his or her understanding processes are directed. To return to the exemplar of a first love, the movement of coming to this understanding also involves a process of meaning.

Not having any previous experience of these things that are clearly happening, a person begins from an initial prejudice which might be overwhelmingly positive emotion. Through languaging the meaning of this initial prejudice he or she is able to determine the satisfactory 'fit' of the prejudice with the emotion. Here the person would come to recognise, through the dialogue of $I$-Thou, that the emotion itself is far more than simply a positive emotion. In so doing the person would develop a greater appreciation of the limitations or meaning of his or her initial prejudice. This process of developing meaning is simultaneously the process of modifying the prejudice. From the initial position of overwhelming positive emotion and the process of seeking meaning of it, the person might develop his or her prejudice to it is more than just being happy, it is being complete, and even further to the prejudice this must be love.

The space in between each of the prejudices in this example embodies that space within which a process of establishing meaning goes on. In other words, meaning is making a determination of how well the initial understanding is sufficient to go on with being. Subsequently, meaning is also a consideration of the need for elaboration of a prejudice. This need for development is a function of the questions that are being asked of it in the dialogue of I-Thou. With this in mind, meaning can be thought of less in terms of understanding per se, and more in terms of what goes on in the to and fro dialogue of the I-Thou relationship. It is this movement that ultimately conditions and sustains understanding itself. This greater appreciation of the notion of meaning sheds more light on the notion of understanding. That is, understanding is the culmination or the outcome of these ongoing meaning-making 
ventures of being human. So much is this the case that understanding as the fundamental comportment of being is conditioned by meaning.

Having considered the inextricable immixture of understanding and meaning, a return can now be made to the consideration of languaging. Recall that languaging is what goes on within the comportment of being. At the core of languaging is recognition of the historicity that is present in all understanding. In other words, languaging is a consideration of the way that time-past and time-present always coalesce in understanding. Here time-present represents the evanescent or fleeting aspects of our existence. In other words, it relates to the 'here and now' characteristics of being in the world. These characteristics are so fleeting that the here and now of having read this is now gone. Time-past on the other hand is the culmination of these evanesced 'here and nows'. However, remaining consistent with the proactivity of the person Hermeneutic Constructivism concerns itself with, this notion of culmination is not to be confused for simply the accumulation of a person's 'here and nows'. Instead, time-past is the culmination of what we have actively made of the 'here and nows' toward which we have directed our understanding processes.

Further examination of this fundamental postulate through the subsequent corollaries will emphasise that time-past is not simply a dogmatism from which each person stands against the world, or a limitation to understanding, instead time-past is an essential precondition of understanding in time-present. A return to the example of a first love is again useful. The initial understanding as an overwhelming positive emotion represents the best that this person could bring to the event of understanding. It is the culmination of what he or she has made of previous here and nows, and therefore comes to represent this person's time-past. It is ultimately the initial understanding from which this person begins the dialogue of languaging. This carrying forward of time-past to future or time-present events of understanding epitomises the centrality of historicity within the Hermeneutic Constructivist position.

One could be mistaken for assuming that it is possible for the person conceived of here to set-down the entirety of his or her historicity or time-past. That is, to bring to language everything that he or she has made of the here and nows that have passed. However, on the contrary, those aspects of time-past that we can access are determined by the prejudice from which we project an understanding toward the world. Here one is reminded of Gadamer's (1960/2003) consideration of how it is that we can come to experience a work of art. We project an understanding toward the art that allows the art to become meaningful. Although we are free to ask questions of what we have illuminated or provided meaning to, these questions always originate from other possibilities of understanding or other possible 
takes on the art. These other possibilities of understanding and therefore the question and answer movement that sustains these possibilities always originate from the $I$. This understanding is therefore always conditioned by our time-past, by what we already have. This notion that our understanding processes are constantly effected by our historicity will be elaborated further in the following chapter. It is enough at this point to note that Hermeneutic Constructivism accepts that it is a person's prejudices that provides a conduit to his or her historicity. Thus in the movement of languaging we progressively open up or gain access to more aspects of our historicity. To explore this idea further the first love example is again useful.

Beginning from the prejudice overwhelming positive emotion this person may ask questions of that emotion in order to understand it better. In so doing he or she is ultimately determining the meaning or the fit of the prejudice to the emotion itself. It is possible that this person might ask the following question of his or her emotion, is it like winning the soccer final? In posing this question the person opens up a different aspect of his or her historicity. And those aspects of historicity that this prejudice opens up toward are now available to contribute to the ongoing I-Thou dialogue. The soccer final may indeed be a positive emotion, in terms of being a part of a successful team, but not in the same way as this overwhelming positive emotion. Thus, through the continued dialogue of languaging, of questioning further and further, this person may gain access to more aspects of his or her historicity. These aspects of historicity that are opened continue to provide the person with further and varied aspects of understanding that sustain the dialogue of languaging and therefore the development of a different understanding.

In order to go on with the theoretical unpacking of Hermeneutic Constructivism, it is important at this stage to appreciate the following summary points about languaging. Languaging embodies the interpenetration of time-past into time-present in all understanding. At its core languaging apprehends the way that the comportment of being toward understanding is fundamentally linguistic in nature. In fact here there is no separation between a person's understanding processes and language. It follows therefore that the person envisaged here is able to be articulate about the qualities of his or her innermost experiences. The coming into language of these qualities of experience, or qualia, is not a special kind of understanding. Instead this coming into language is the very condition of qualia itself.

Events - Although briefly outlined above, the implicitly proactive consideration of the person that is embodied by the notion of an event has widespread implications for a consideration of experience. An event is determined by those aspects of the world toward which we direct our 
understanding processes or our languaging efforts. It is these aspects of the world that we therefore come to understand or make something of. Thus, as early as the fundamental postulate, Hermeneutic Constructivism emphasises that experience is more than simply a being there in the here and now of time present. Instead, to have an experience of the world always requires the person makes something of that event.

\section{Elaborative Corollaries}

The Fundamental Postulate has established that the individual person of Hermeneutic Constructivism through the medium of language and the movement of languaging itself is a proactive constitutor of the world that he or she comes to understand. The following eleven corollaries are considered to logically follow from and augment this Fundamental Postulate.

\section{PREJUDICE COROLLARY - A PERSON DEVELOPS DIALOGICALLY A SERIES OF ANTICIPATORY PREJUDICES THAT GUIDE HIS OR HER LANGUAGING PROCESSES.}

The unpacking of the concept of languaging thus far could be considered to be little more than a form of unwieldy relativism. This would be a position where a person simply brings the world to understanding in the way he or she envisages as appropriate from moment to moment. However we must recall that it is the prejudice itself that provides the spectrum within which the movement of languaging is ultimately determined. That is, the established pathways or guides that our prejudices provide operate to control our understanding processes. The human comportment toward understanding is therefore not a simple 'anything goes' relativism; instead it is determined by the possibilities that our prejudices provide as fundamentally historical structures. It is therefore and always the prejudice that provides the light by which the world is illuminated. In fact it is the prejudice that allows human being to have a world at all.

Develops - Once again this captures the proactive nature of person the languager. The term here also emphasises the way that our prejudices are always open to evolution through the ongoing dialogue of languaging. That is, the to and fro dialogue resembles the I-Thou relationship already outlined.

Dialogically - Here a dialogue implies a to and fro movement that resembles the structure of question and answer. It is the prejudice that provides entrée to the dialogue of languaging as well as sustaining the dialogue itself. This is because the prejudice ultimately determines those aspects of a dialogue that can become meaningful. For example, if one was to attempt to make sense of a shape that appeared within the water while swimming at the beach, the prejudice not all man eating fish are threatening would not meaningfully allow a dialogue 
concerning watermelon to ensue with a fellow swimmer. ${ }^{14}$ Although a dialogue always originates from a prejudice, it is the dialogue itself that simultaneously conditions the ongoing development of the prejudice. In other words, given that the prejudice has its being in language, the way in which the prejudice comes to language is not different from the prejudice itself. Therefore the initial prejudice from which the dialogue of languaging originates always has the potential to become something different. In turn this becoming something different represents the development of a different understanding. In fact this development of an initial prejudice elaborates a little further what the human comportment of being toward understanding actually is. The different or rather developed prejudice itself has the potential to be the initial prejudice from which languaging begins in future events of understanding.

Series - All understanding for the person that Hermeneutic Constructivism envisages originates entirely and only in the light provided by a prejudice. However it is not considered possible for this person to develop a single prejudice that is able to bring sufficient meaning to or to illuminate every event that the world has to offer. As a function of this, we develop a series of prejudices. These prejudices are related to each other and together form a system of prejudices that provide the grounds for our anticipating the world. The nature of this relationship between prejudices will be explored in more depth through The Organisation Corollary. Importantly, although a single prejudice is incapable of throwing sufficient light upon every event of the world, in order to bring an event to understanding the light of an ineffective prejudice is indeed better than the darkness of no prejudice at all.

Anticipatory - The Fundamental Postulate suggests that our understanding processes are guided. These guides are in fact our prejudices, and are established prior to an event of understanding. Therefore the prejudice is fundamentally anticipatory in nature.

Prejudice - As previously outlined, a prejudice represents an abstraction of what has been made of time-past and at the same time conditions understanding in time-present. Thus the prejudice is the very condition of the human comportment toward understanding and therefore of languaging itself. The prejudice is therefore an essential prejudgement that provides entrée to the dialogue of languaging and yet simultaneously has its being in this same dialogue. Despite the fact that the prejudice only comes to understanding in the dialogue of languaging, it is not a structure that we can simply stand back from and contemplate. Instead the prejudice is a fundamentally ontological structure that is essential

\footnotetext{
${ }^{14}$ The degree to which the prejudice is able to exert an influence on the dialogue of languaging is explored further in this and later corollaries.
} 
for being and can only come to be understood in the dialogue of being. A look at the way that the prejudice sustains the dialogue of being will facilitate a deeper understanding of the prejudice itself.

There can be little doubt that language fills itself with meaning that goes well beyond simply what is said. ${ }^{15}$ In other words, the meaning that is embodied by a phrase is far more than the literal meaning of the words themselves. The metaphor or idiom is exemplary of this notion. The statement a bird in the hand is worth two in the bush, for instance, embodies the idea that already having something, even if only small, is better than risking losing it in the pursuit of more. The language of this idiom carries with it a clear example of the inner dimension of meaning that is inherent within language. ${ }^{16}$ Meaning, then, is far more than simply what is said. But from where does this inner dimension of meaning come from? How does the prejudice that we project towards the thing that is to be understood fill itself with meaning in order to sustain the dialogue of languaging?

The inner dimension of meaning of the prejudice comes from our time-past. Timepast is the culmination of those somethings that we have made of previous events of understanding. It is these somethings that represent what we already have, our understanding thus far, and therefore what we can now bring to understanding in time-present. However we do not simply reflect upon these understandings and then choose between them. Instead these understandings are accessed only by our prejudices and therefore only through the dialogue of languaging. Worded another way, our prejudices effectively provide a conduit to these somethings or our understanding thus far. It is therefore those aspects of our time-past, ultimately our historicity, that are accessed by a specific prejudice that operates to fill that prejudice, and therefore the dialogue of languaging, with meaning. It is this concept of filling itself with meaning that allows the prejudice to establish and maintain the I-Thou dialogue of languaging. In follows, therefore, that if being is the movement toward understanding, then the direction of languaging is always toward the evolution of the initial prejudice. That is, languaging is always directed towards a different or better understanding through which to illuminate an event in the future.

The discussion thus far has progressively elaborated the notion of the prejudice as a fundamental and inextricable precondition of understanding. In light of this, the way in

\footnotetext{
${ }^{15}$ Here are present the hallmarks of Gadamer's elucidation of the speculative nature of language, where language carries meaning that goes well beyond simply that which is said. For a more complete discussion see the section in Chapter Five titled The Speculative Nature of Language.

${ }^{16}$ One is reminded here of Gadamer's (1960/2003) suggestion that "meaning thus reduced to what is stated is always distorted meaning" (p. 469).
} 
which Hermeneutic Constructivism conceptualises qualia, as the qualities of our internal experience can now be unpacked further. Although the notion of experience will be elaborated further in this chapter and the chapter to follow, enough has been said up to this point in order to be clear that experience requires proactivity on the part of the experiencing person. This proactivity has as its condition the projection of a prejudice or initial understanding toward the thing that is to be understood. As established above, all understanding occurs entirely in the light of a prejudice. This is not different when the thing that is to be understood is our internal understanding processes. It follows, therefore, that if qualia are the qualities of our internal experience, then they too are conditioned by our prejudices. After all, the very notion that something has a quality indicates that something has been made of it. To be more specific, in order for something to have a quality or a certain characteristic means that something has been made of it through the dialogue of languaging. In order for us to make something of a thing, we must project a prejudice and understanding toward it. It is therefore the prejudice that is ultimately the condition of our internal experience. It is the prejudice that allows us to have qualia at all. Here then qualia are inextricably linked to not only the prejudice(s) that condition but also language. Thus qualia are not different from the way in which they can come to language as a prejudice.

Guide - Although described within the Fundamental Postulate it is important to be clear that these guides as prejudices are not determinant of the possibilities of understanding as a dogmatism or a reduction to the possibilities of understanding. Instead these guides are the initial positions from which we always begin the to and fro dialogue of languaging. Recall that the comportment toward understanding is always in the direction of the evolution of our prejudices. Therefore each prejudice or guide always has the predisposition toward being different as its fundamental nature.

HORIZON COROLLARY - EACH PREJUDICE PROVIDES THE HORIZON WITHIN WHICH A THING TO BE UNDERSTOOD CAN MEANINGFULLY COME TO UNDERSTANDING.

Enough has been said at this point to be clear that the prejudice is determinate of the possibilities of the way that the world can come to understanding through the dialogue of languaging. This corollary provides a further unpacking of what is meant by this determinism and therefore the essential nature of the prejudice itself. This corollary is recognition that each prejudice carries with it a horizon. The notion of a horizon here embodies what it is that can be illuminated and therefore seen from a particular vantage point or prejudice. In other words, this corollary establishes that each prejudice is only able to illuminate or provide understanding to a finite number of events. 
Each - The use of this term establishes that each prejudice has its own horizon of understanding. Each person is free to move within the spectrum of the horizon that a single prejudice provides. Equally, through the dialogue of languaging, a person can also move into the horizon of a different prejudice in order to achieve a different perspective or to project a different light upon that event that is to be understood.

Horizon - Here Gadamer's (1960/2003) notion of a horizon is reinstated. He contends that the horizon is all that is available to be envisaged from a particular vantage point. For Hermeneutic Constructivism, then, the horizon represents the totality of those aspects of the thing that can meaningfully come to understanding from the vantage point of a particular prejudice. Thus the horizon of the prejudice is the embodiment of everything that one already has about the thing to be understood that can be accessed by that prejudice. In line with this, as the prejudice itself is developed or evolves so too does its horizon or those aspects of timepast that it provides access to.

Up to this point, the term time-past has been used to capture that something a person has made of his or her understanding processes in previous here and nows. However a more focused consideration of the prejudice requires more precise language. Thus, from here on, those aspects that we already have about the thing to be understood are referred to as authentic consciousness. Although this notion will be elaborated in greater depth in the following chapter, it represents more effectively the historical effect that the comportment toward being has and the way in which we are affected at every moment in the present comportment by that something that we have made of past here and nows. Thus, in terms of the horizon, and to be more precise, a horizon represents that aspect of authentic consciousness to which the prejudice provides access.

Within - The use of the term within embodies the idea that a horizon is not a fixed position from which human understanding originates. Rather the horizon is a spectrum that carries possibilities of understanding. It is a spectrum within which each person can move in coming to an understanding.

A thing To Be Understood - Although the fundamental comportment of being is toward understanding, this phrase captures the way that understanding is only of the thing toward which we direct our understanding or languaging processes.

Meaningfully - It has been established that understanding represents the lasting elements of what we make of events of the world. Moreover, in coming to that understanding, we determine the meaning or the fit of our prejudices to the event at hand. What is at stake here, 
then, is the degree to which the prejudice is able to meaningfully bring the world to understanding. More specifically that the prejudice's fit is sufficient in order to go on being in the world.

\section{ANTITHETIC COROLLARY - EACH PREJUDICE HAS AN IMPLICIT ANTITHESIS BEYOND WHICH} ITS UTILITY IS LIMITED.

It has been established that each prejudice has a finite spectrum with which it can be considered useful for bringing the world to understanding. This spectrum constitutes what it is that can be understood from the particular vantage point of a specific prejudice. In fact this spectrum is the horizon of the prejudice. As indicated above, the horizon of each prejudice constitutes the spectrum within which a person can move to bring a thing to understanding. Being conceptualised in terms of a spectrum, it follows that this spectrum ultimately has limits. The purpose of this corollary is to disclose further the nature of the prejudice itself. In so doing, this corollary seeks to emphasise that each prejudice is a spectrum and therefore more than merely a single fixed position from which to illuminate the world understandingly. This spectrum consists of the prejudice that is ready at hand or the Evident prejudice and its implicit opposite the Antithetic prejudice. In other words, each prejudice, as part of its fundamental structure, has its own Evident prejudice and Antithetic prejudice. In combination these structures embody the context of the prejudice. It is important to appreciate that the context of the prejudice simply represents the bi-polar structure of the prejudice itself. The horizon on the other hand represents those aspects of authentic consciousness that can come to understanding along the spectrum or within the context of the prejudice. In other words, the horizon is the totality of what can come to understanding from our authentic consciousness as that spectrum between the Evident prejudice and the Antithetic prejudice.

The importance of the antithetic prejudice cannot be overlooked and is attributed to the work of Hegel (1807/1967) and Kelly (1955). Hegel (1807/1967) suggested that there is no preconceived position or thesis that is devoid of an inherent antithesis. ${ }^{17}$ Hegel believed that by establishing this dichotomy, of thesis-antithesis, it is possible for a person to identify

\footnotetext{
${ }^{17}$ Although the model most often associated with Hegel's dialectic comprises three separate stages: a thesis, an antithesis, and a synthesis, Hegel himself did not actually use this formulation. Instead Hegel used the terms: abstract, negative and concrete throughout his work in order to capture the movement of his dialectic (Rockmore, 2003). Irrespective of terminology, the central idea is that an initial thesis is always open to the challenge of being too abstract and is therefore open to the possibility of negation. Here we move dialogically between the two points - the thesis and its antithesis - by a process Hegel termed Aufhebung, where we move from an initial position to another position. More specifically, this term captures the way that one preserves some elements while changing others in the process of overcoming the limitations that have been identified within the initial thesis. The dialogical movement between thesis and antithesis succinctly captures the role that the antithesis plays in Hegel's dialectic for human understanding (Gadamer, 1976).
} 
sources of conflict between these two positions and to then, develop a synthesis or a means of overcoming these sources of conflict. In line with this, Kelly (1955) suggests that in order to understand better what a person means, we need to look at what he or she does not mean. ${ }^{18}$ Equally Hermeneutic Constructivism takes the view that in order to appreciate better those aspects of the world that a person's prejudice can illuminate, we need to apprehend those aspects of the world that the prejudice does not illuminate. In other words, in order to understand the way in which a person uses an Evident prejudice to bring an event to understanding, we need to appreciate those aspects of the world that this evident prejudice cannot provide meaningful understanding to. That is, we need to gain an understanding of what the Evident prejudice is not.

Implicit - Although each Evident prejudice always has the possibility of an Antithetic prejudice, in the everyday use of our prejudice systems we may not in fact become aware of this Antithetic prejudice. The Organisation Corollary will emphasise that in everyday understanding we simply evanesce from one Evident prejudice toward another in the comportment of being. It is for this reason that we do not find ourselves grappling with questions about our prejudices, questions that might involve such matters as what a prejudice is not. Despite this, Hermeneutic Constructivism takes the view that the antithetic nature of a prejudice is implicit within the structure of the prejudice itself. That is, the Evident prejudice itself represents an understanding or a take on the world, a thesis if you like. In addition, every thesis has an implicit antithesis or a position at which that thesis is considered to be of little use. Thus although we may not come up against these Antithetic prejudices in the comportment of being, they represent those aspects of the world that an Evident prejudice cannot illuminate and are therefore implicit within the structure of each prejudice itself.

Antithesis - The notion of antithesis has its origins here in the idea that if we are able to identify those aspects of the world that we can envisage, then we are equally able to identify those aspects that we cannot envisage from the vantage point of a specific prejudice. In order to appreciate this we need to first review the role of the Evident prejudice in languaging. In the comportment of being we reach toward an event of understanding from the perspective of our Evident prejudice, the understanding that we already have and which readily comes to hand in understanding. It is from this initial position that the dialogue of languaging begins, a dialogue that is itself informed at every moment by those aspects of authentic consciousness

\footnotetext{
${ }^{18}$ The following passage from Kelly (1955) highlights the value of this manoeuvre. Considering the notion of 'respect' as one end of a bi-polar construct, Kelly (1955) suggests; "we need to look at both ends of it if we want to know what it means to him. We cannot understand him well if we look only at the similarity - respect - end of the dimension. We cannot understand what he means by 'respect' unless we know what he sees as relevantly opposed to 'respect"' (p. 71).
} 
that the evident prejudice provides access to. However those aspects of authentic consciousness that we gain access to are determined not by the Evident prejudice alone, but by the broader context of the prejudice itself. Recall here that the spectrum of the possibilities of understanding that we have available to us, from the vantage point of a specific prejudice, are located between the Evident and Antithetic prejudice.

The dialogue of languaging has already been disclosed as following the movement of question and answer as outlined above as the I-Thou relationship. One will recall that the formulation of ongoing questions, in order to sustain this dialogue, is conditioned by our authentic consciousness and more specifically by those aspects of authentic consciousness that a particular prejudice provides access to. Thus in order for a question to be developed and then posed of the thing that is to be understood, something must already be understood about it. That is, we must already have some understanding of the thing if we are to generate suitable questions of it. There are times when we may find it difficult to engage in a dialogue, perhaps because we cannot find an appropriate question with which to enter into a dialogue itself. An appropriate question would apprehend what it is that we already understood, as well as open up those aspects that we do not. However when we struggle to generate such questions, it indicates that our prejudice, our precondition of understanding, is not sufficiently developed. It is therefore at the point where we find it difficult to develop questions that we can pose of the thing that is to be understood, that the limits of the prejudice are being approached.

In order to apprehend further this notion of antithesis we must appreciate the nature of the prejudice more deeply. The prejudice is not a structure that is reminiscent of a series of separately defined 'cells'. That is, we do not merely go into one cell to envision the world, and then go out of it in order to then go into another cell. In fact, the bounds of the prejudice are much less defined, and prejudices are constantly coalescing with each other. It is for this reason that during everyday dialogue we do not often find ourselves asking what now?, having reached the limits of the context of our prejudice. Instead, in everyday being, each prejudice simply evanesces into another prejudice from which further questions may springforth, and indeed do, in order to sustain the dialogue of languaging. However, having established the antithetic nature of the prejudice as implicit within its structure, Hermeneutic Constructivism suggests that we can be clear about the point at which our prejudice would otherwise evanesce toward another prejudice in understanding. This point is the point at which we can no longer develop meaningful questions to be asked of the thing to be understood. It is this point that therefore represents the point at which the illuminating capacity of the specific prejudice grows dim. 
Beyond - It has been disclosed that the world exists outside of the way in which it comes to be understood through our prejudices. Thus there is always the possibility of envisioning the world differently from the possibilities of understanding that our prejudices provide at any one point. The antithetic nature of prejudice therefore represents that point beyond which a prejudice's utility is limited. Worded another way, the antithetic point is the point beyond which the person can no longer develop a suitable question to be posed of the thing to be understood and is therefore the point at which this prejudice cannot suitably illuminate the thing in understanding.

Utility - The utility of a prejudice is a function of its usefulness. In other words, how useful it is in bringing the world to sufficient understanding in order to go on with it.

Limited - At the core of the human comportment toward understanding is the prejudice. So much so, that in the absence of a prejudice the world would be a swirling blur of sensory stimuli. In order to go on in the world we must ask questions of it. The capacity to develop these questions is progressively more limited as the person approaches the antithetic aspect of the context of the prejudice. Thus, at the antithetic end of the prejudice, the capacity to illuminate a particular aspect of the world is limited.

\section{ORGANISATION COROLLARY - EACH PREJUDICE BECOMES AN INTEGRAL COMPONENT OF A} GREATER DIALOGICAL SYSTEM OF PREJUDICES.

It has been mentioned above that in an event of understanding, as we approach the antithetic end of the spectrum of a prejudice's context, a different prejudice arrives. This different prejudice subsequently provides access to different aspects of our authentic consciousness. In so doing, it provides the possibility of developing different and further questions that can be asked of the thing to be understood. The Organisation Corollary recognises that in the absence of a sense of organisation or lawfulness to our prejudices, we would be incapable of finding any sense of consistency in what goes on around us and subsequently we would not be able to bring the world to meaningful understanding. Thus at the core of the Organisation Corollary is a view that each prejudice is dialogically related to other prejudices, thereby creating a system of prejudices. It is this system from which all understanding - the comportment of being - has its origins. As such this system represents all that we currently understand about the world as well as the possibilities that we have available to us for envisaging the world. In light of this, it is this system of prejudices that represents the Self. In fact a person's system of prejudices represents more completely his or her Self than any single prejudice alone. 
This system of prejudices is a system of related structures within which we move dialogically in order to bring the world to understanding. This corollary is reflexive of the way in which we tend to move dialogically from an initial prejudice or understanding into the spectrum of another prejudice with a degree of consistency. Here the use of the term 'degree' does not mean that it is by mere happenstance that we have a tendency to reach almost the same prejudice in different instances of understanding. Instead, the term degree reflects the way in which the prejudice that we tend to evanesce toward is capable of evolving. That is, the prejudice that we evanesce toward in the movement of languaging in one instance may indeed be the same prejudice in a future instance, albeit, in the here and now, a different prejudice as a function of having evolved through the dialogue of languaging itself. In terms of the greater system of prejudices, then, when the context of the prejudice changes as a function of this evolution, so too does the utility of the prejudice and therefore the point at which it evanesces toward a different prejudice. The Organisation Corollary is therefore a consideration of the way in which our system of prejudices embodies a degree of consistency and lawfulness.

In maintaining a sense of reflexivity it is important to apprehend the way in which our beliefs and values influence our understanding. Here adopting the structure elaborated within the Psychology of Personal Constructs, Hermeneutic Constructivism elaborates the relationship between prejudices in terms of superordinacy and subordinacy. ${ }^{19}$ Therefore it is suggested that a person's prejudices are situated in terms of a hierarchy, with superordinate prejudices exerting influence over those prejudices that are subordinate to them. Following this position, there are prejudices that are of the highest order within a person's system and are therefore core prejudices. It is these core prejudices that embody our most central values and beliefs about the world. Given the centrality of such beliefs to the ongoing stability of our everyday lives, it is these core prejudices that are most resistant to radical change. ${ }^{20}$ It follows, therefore, that these core structures exert an influence over the evolution and development of all subsequent prejudices within a person's greater system of prejudices.

Integral Component - The word 'integral' captures the type of relationship envisaged of our greater system of prejudices. Such a relationship embodies the way that each prejudice exerts or is able to exert an influence on those prejudices that are dialogically related to it. As

\footnotetext{
${ }^{19}$ For a consideration of the notion of these terms in Kelly's work please review Chapter Three of this thesis.

${ }^{20}$ A prime example of a core prejudice that yields a high level of influence upon a person's greater system of prejudices is religion. For some the belief in a divine being and the will to follow a determined path of human existence significantly influences the way in which 'everyday' or subordinate prejudices are developed.
} 
outlined above, the evolution of a prejudice subsequently affects the point at which that prejudice will evanesce towards another prejudice in the future.

Greater - It has been established that each individual prejudice exists within a system of prejudices. The term 'greater' is used to apprehend the complexity of this system. As a function of this, it is not possible for us to simply stand back from our prejudice system in order to recognise upfront those prejudices that are productive from those that are not. Instead, we only come to know our prejudices within the dialogue of understanding itself; the dialogue of languaging.

Dialogical - Dialogical relates to the ability of the person that Hermeneutic Constructivism envisages to bring to language his or her greater system of prejudices. Importantly, this does not conflict with the contrary suggestion made above. Instead it indicates that in the dialogue of understanding itself - a dialogue that takes the form of question and answer - we can bring to language those prejudices that we have a tendency to evanesce toward during the movement of questioning itself. In other words, it is only in the dialogue of languaging that we can be articulate about the prejudice from which we envisage a thing that is to be understood.

System - It is important to establish that the use of the term 'system' is not to be confused with a mechanical system that can undeniably represent an integral relationship between its working parts. Although here the term system does embody the notion of a series of parts that function together to form a complex whole, it is a system that can change and evolve - a dynamic system.

\section{INDIVIDUALITY COROLLARY - PERSONS DIFFER FROM EACH OTHER BY THE WAY THEY}

\section{LANGUAGE THEIR PREJUDICES.}

The fundamental postulate is clear that anticipatory pathways that are formed by the ongoing dialogue of languaging guide each person's understanding processes. The Individuality Corollary reaches forward from this position to emphasise the way that these anticipatory pathways - referred to as prejudices - come to language, and in so doing provide a basis for conceptualising individual difference between people. Here, as we know, the prejudice is the embodiment of what has been made of or understood about a thing, through the dialogue of languaging. It is from the vantage point of the horizon of a prejudice that we reach toward the thing that is to be understood. Through the dialogue of languaging that is sustained by authentic consciousness, each of us is carried along in the comportment of being. 
Importantly we are each carried along in a different and unique way as a function ultimately of our understanding processes - languaging.

As outlined above, language carries with it the possibility of meaning that goes well beyond simply what is said. That is, language is fundamentally speculative in nature. It follows therefore that the fundamentally linguistic nature of our understanding processes means that each of us differ in the way in which we bring the world to language. What is more, in a situation where two people use the same language to represent an understanding, the inherently speculative nature of language means that each individual person may indeed mean something entirely different. When provided an opportunity to language further the meaning of his or her understanding, the inherently idiosyncratic meaning embodied by the person's words, for that person, is able to come to the fore.

Although the Individuality Corollary is clear that each person's meaning making processes are indeed fundamentally individual as a function of the essentially speculative dialogue of languaging itself, Hermeneutic Constructivism is nonetheless reflexive to the possibility that people share experience. ${ }^{21}$ However 'sharing' here is not having the same experience. Instead it is a process of envisioning the possibility of another person's experience, a possibility that will be emphasised further in later corollaries.

The languaging of their prejudices - The Fundamental Postulate establishes that the movement of languaging underpins the business of being human as a comportment toward understanding. This corollary seeks to further amplify this position by considering what it is that we actually language in coming to an understanding. As outlined, the prejudice provides the initial position from which we envisage the world. As well as this, the prejudice represents the possibilities of what can meaningfully come to understanding through its dynamic and essential relationship with authentic consciousness. The prejudice is therefore the essential precondition of the comportment of being, and as such is the essential structure that allows us to discriminate between people. That is, the prejudice is what makes you, you, and me, me, inherently unique people. Therefore the prejudice is the fundamental structure upon which people can be considered to differ.

The inextricability of the prejudice from language, and an earlier appeal to a speculative conceptualisation of language, informs a view that we each develop an

\footnotetext{
${ }^{21}$ This conceptualisation of language as speculative is consistent with Gadamer's (1960/2003) elucidation. A review of this work can be seen in Chapter Five. Here Gadamer suggests that although language is finite, in that there are ultimately a given number of words available for use, these words carry infinite meaning. It is upon this basis that the idiosyncratic position is being established here.
} 
idiosyncratic system of prejudices from which to envisage the world. In so doing, the likelihood that two people would bring to bear an elaboration of their prejudices, and the connections that these prejudices make within their greater system of prejudices, in the same way, is highly unlikely to say the least. Thus the ontological nature of the prejudice, together with the speculative conceptulisation of language, provide grounds for suggesting that each person is fundamentally idiosyncratic in his or her comportment toward understanding.

EXPERIENCE COROLLARY - TO THE EXTENT THAT ONE LANGUAGES AN EVENT OF THE WORLD HE OR SHE CAN BE SAID TO HAVE AN EXPERIENCE.

Inherent within the Fundamental Postulate and the notion of languaging is a proactivity on the part of the person. The Experience Corollary extends this position by suggesting that experience is not simply a function of a person being present while the world goes by. Instead experience is a function of a person's proactivity. In other words, experience is a function of what the person makes of their presence within the world. As described previously, we always begin from an initial prejudice and through the dialogue of languaging we evolve or develop that prejudice to a different position in order to anticipate future events of understanding. Through this dialogue the prejudice itself becomes an essentially different prejudice. It is this evolution of an initial prejudice to a different prejudice that represents genuine experience per se. In such a conceptulisation, experience is not considered in terms of an off/on modulation. Instead it takes the form of degrees or depths of experience, determined by the extent to which a person languages an event. That is, the more that we are able to engage with different aspects of our prejudice system in the dialogue of languaging, then the more widespread the possible effect that the dialogue of languaging can have on our greater system of prejudices. The greater the effect on a person's prejudice(s), the greater the depth of experience that can be considered to have occurred.

To The Extent - The use of this phrase provides entrée to the idea that experience is directly related to the extent with which a person engages in the dialogue of languaging. This not only recognises the centrality of human proactivity in Hermeneutic Constructivism, it also reaches toward experience in terms of a depth of effect.

Experience - Experience is what ultimately results from languaging an event of the world. From an initial understanding or prejudice we reach toward the world in order to understand it. When we begin to ask further questions of the thing that is to be understood, we are actually recognising that a better understanding is possible, or needed, in order for us to satisfactorily go on being in the world. Thus it follows that we begin the dialogue of 
languaging in order to develop these initial prejudices. That is, we have an experience, and in so doing develop our understanding for future events of understanding.

For Hermeneutic Constructivism 'experience' has an inherently productive and yet negative quality. In the dialogue of languaging a thing to be understood, when we apprehend an aspect of that thing that is not already well anticipated by our prejudices, we are compelled by the comportment of being to develop that prejudice. ${ }^{22}$ Thus, experience is here negative given that we recognise that an initial understanding is not sufficient to go on in the world. However, at the same time, experience is itself productive in light of the possibilities opened up for the development of our understanding. Experience allows us to understand a thing better or differently and in so doing can change the way that we anticipate similar events in the future.

\section{PASSIVE COROLLARY - A PERSON IS AUTONOMOUS WITH REGARD TO BEING PROACTIVELY INACTIVE WITH REGARD TO HIS OR HER LANGUAGING EFFORTS.}

The person with whom Hermeneutic Constructivism concerns itself with is considered to be equally proactive in his or her decision not to be proactive. In other words, although the comportment of being is always toward understanding - a process that has been outlined here as requiring an inherent proactivity on the part of person - this same person has the possibility of being proactively inactive. The Passive Corollary is therefore reflexive of the way in which we each have the capacity to simply be, and to allow the happenings of the world to 'wash over' us without having to make something of them. Invariably, however, the mode of an event may change a person's degree of proactivity. For example: a baby crying, a knock at the door, or the ring of a telephone, are some obvious examples that may indeed alter a person's degree of proactivity. However, what is important to apprehend here is that a person is able to be passive towards the happenings of the world and his or her efforts to make something of them.

Autonomous - This term accents the genuinely proactive nature of the person who is apprehended by Hermeneutic Constructivism. This person is autonomous with regard to those happenings or events towards which he or she directs his or her understanding processes. Moreover, this person is also autonomous with the degree to which he or she engages in the process of languaging per se.

\footnotetext{
${ }^{22}$ This notion is most consistent with Gadamer's (1960/2003) elucidation of experience. For an overview of this refer to Chapter Five.
} 
Proactively Inactive - This statement makes clear that for Hermeneutic Constructivism the steady state of the person is one of proactivity. So much so that a state of passivity is in fact a specific function of this proactivity. Moreover, the idea that a person is proactively inactive is not an all or nothing approach. Instead we always actively decide what we will be passive towards. A person could proactively adopt a position of passivity with regard to his or her own incessantly screaming child, given that a partner is taking responsibility for the child, however at the same time be deeply engaged in a dialogue with a colleague or friend or novel.

SOCIALITY COROLLARY - TO THE EXTENT THAT ONE PERSON IS ABLE TO SHARE - THROUGH LANGUAGING ABOUT A THING TO BE UNDERSTOOD - ASPECTS OF HIS OR HER HORIZON WITH ANOTHER INDIVIDUAL, THAT PERSON IS CONSIDERED TO HAVE ENGAGED IN A SOCIAL RELATIONSHIP.

At this juncture one could be forgiven for believing that Hermeneutic Constructivism apprehends a theoretical conceptualisation of the individual going about the business of being human without the presence of other like individuals. On the contrary - and remaining theoretically reflexive - going about the business of being human always involves other proactively languaging people. It is important to appreciate that the dialogue of languaging a thing in the world requires the same processes when the thing to be understood is another languaging person. That is, the dialogue of languaging aspects of the world that cannot speak, aspects that therefore take the form of a Thou in an I-Thou relationship, follows the very same structure as the dialogue that goes on between people.

This corollary establishes that simply being present within a world with other people does not represent a social relationship. Instead a genuine social relationship is the sharing, between people, of an aspect(s) of his or her 'inner outlook'. Essentially a social relationship is an opportunity to share the way we each see the world. Therefore the Sociality Corollary locates the social relationship as a sharing of an aspect(s) of our greater system of prejudices with another person. The term sharing is important here. In a genuine social encounter, each individual person is provided an opportunity for his or her prejudices or understanding to be given more or less equal consideration within the to and fro dialogue about the subject matter of focus. Here aspects of the each individual person's prejudice system and the subsequent horizon of these prejudice(s) coalesce and in so doing create a social relationship.

Share - The basic element that allows one person to share with another person his or her languaging processes is language itself. Hermeneutic Constructivism has gone to great lengths to establish language as the medium that conditions and therefore provides the very possibility that we can have an understanding of the world at all. The comportment of being 
toward understanding has been unpacked in such a way that each of the essential processes and structures that are essential to this comportment also have their being within language. In so doing, the person envisaged by Hermeneutic Constructivism is able to bring to language to share or to be articulate about - his or her view of the world for and with another person.

Languaging About - As already mentioned, although languaging has been captured up to this point as a fundamentally inner activity of the person, the dialogical nature of languaging itself is not different when it goes on between people. Here a metaphorical space is created between two languaging people that provides an opportunity for a 'languaging about' the thing that is or, rather, becomes the subject matter of the dialogue. Thus in any genuine dialogue this thing is placed - again metaphorically - within the space between people who are seeking to apprehend a better or different understanding of it.

In a genuine dialogue each person externalises aspects of his or her prejudice system and concomitant horizon in the dialogue of languaging for consideration by the other person(s). This opens up an invitation to the other person to engage in a dialogue and to externalise aspects of his or her inner outlook. If indeed this exchange is a genuine dialogue it will take the form of a to and fro movement of question and answer. Here each interlocutor languages the prejudice(s) of the other person by asking questions and seeking answers to those questions. By way of a series of ' $a h a$ ' moments, these interlocutors reach agreement about the adequacy or satisfactory nature of the understanding that has arisen in the dialogue, in order to go on with the dialogue or to cease it completely.

It follows that the more of a person's prejudice system that he or she brings into a to and fro dialogue with another person, the greater the possibility of having those prejudices developed by the dialogue itself. Here a person may find that an initial prejudice is inadequate for anticipating a certain aspect of the world that the other person has understood differently, and as a result this initial understanding has evolved as a function of the dialogue. Therefore it is this essentially circular movement of question and answer that epitomises a genuine dialogue, providing an opportunity to develop a person's system of prejudice(s) and his or her subsequent horizon(s).

Aspects of One's Horizon - It has already been established that the prejudice and its subsequent horizon have their being in the dialogue of languaging and as such can only come to be understood in an event of understanding itself. Thus, in terms of this turn of phrase, only in this dialogue of languaging do aspects of our horizon come to be understood. Worded another way, it is not possible for us to stand back from our own horizon and elaborate it completely, independent from the ongoing question and answer movement of languaging. 
Social Relationship - From the position of theoretical reflexivity, we can lay claim to many and varied situations in which we have engaged in a dialogue with another person, but which have not resulted in us coming to know more about that person or the subject matter of focus. Thus it would be a philosophical faux pas to assume that this circular dialogue always results in one person understanding another person or the subject matter better than he or she did initially. Although each of us have borne witness to failed ventures of languaging about a thing to be understood with another person, it would be fool-hardy to comprehend even these failed ventures as being less than a social relationship. Thus, for Hermeneutic Constructivism, the externalisation of a person's languaging processes towards another person, who acknowledges the invitation as such - as an externalisation of one's languaging processes - is the basic requirement for a social relationship.

SIMILARITY COROLLARY - TO THE EXTENT THAT ONE PERSON BRINGS TO LANGUAGE HIS OR HER SYSTEM OF PREJUDICES IN A MANNER COMPARABLE TO THAT OF ANOTHER, HIS OR HER LANGUAGING PROCESSES CAN BE CONSIDERED SIMILAR TO THAT OF THE OTHER PERSON.

In keeping with the principles of the Antithetical Corollary, it would be incommensurate to outline an Individuality Corollary - encompassing the way by which people differ from each other - without a consideration also of that by which people can be considered similar. Hermeneutic Constructivism has emphasised the importance of gaining not only an appreciation of the words that are exteriorised, but also of the connections these words make within authentic consciousness for the individual person. It is the elucidation of these connections between a person's prejudices and the broader meaning of these prejudices from authentic consciousness that represents most squarely the 'inner outlook' of the individual person.

The comportment toward understanding is conditioned by the prejudice which opens up the horizon or that aspect of authentic consciousness that operates to fill the prejudice with meaning. The nature of the prejudice as an essential structure of understanding and its elaboration from authentic consciousness provide fertile ground for apprehending the inner outlook of the individual person. In addition, the opening up of a person's horizon provides a productive means by which to make judgments concerning the degree to which that person can be considered similar to another. It is important to recognise here that a similarity between the prejudice(s) of two people is not to be confused as a similarity in languaged meaning. That is, the speculative nature of language is a recognition that people could use the same words and yet mean vastly different things. In keeping with the Experience Corollary, it would be possible for two people to have the same experience and yet bring that experience to language in very different ways, and vice versa. Instead, for Hermeneutic Constructivism, 
a similarity in the languaging of a prejudice is merely recognition that two people can be considered similar with regard to their languaging processes.

Comparable - The Similarity Corollary could be considered a reminder that when a person brings his or her prejudice to language in a manner that is similar to another person, this is not necessarily that they carry similarity in meaning. Worded another way, a similarity between individual people on the basis of what is said is not necessarily a similarity of the meaning that lay behind their prejudices. That is, the speculative nature of language and what it is that a specific prejudice opens up toward in the authentic consciousness of the particular person are ultimately what determines what the prejudice itself means.

The Organisation Corollary has made it clear that each prejudice is determined by, and is perhaps determinant of, at least one other prejudice, thereby forming the greater system of prejudices. Given the inherently dynamic and complex nature of prejudice systems, Hermeneutic Constructivism is skeptical of the likelihood that a similarity in the languaging of a prejudice would extend beyond one or two prejudices. Equally it is possible that two people could develop the same meaning of an event and yet adopt a seemingly different series of prejudices. Thus the extent to which two people bring their prejudices to language in a manner that is comparable, supports a similarity in their languaging processes but not necessarily a similarity in experience. ${ }^{23}$

\section{APPLICATION COROLLARY - TO THE EXTENT THAT AN INDIVIDUAL IS ABLE TO APPLY THE} HORIZON OF THAT WHICH IS OTHER TO THE HORIZON OF HIS OR HER OWN PREJUDICE, HE OR SHE WILL HAVE ACHIEVED A FUSION OF HORIZONS.

It must be recalled, at this point, that Hermeneutic Constructivism takes the view that understanding can never be complete or perfect as an understanding of a thing 'in-itself' . Instead what can be achieved is an understanding of the thing as an understanding 'of-itself'. That is, the way that the thing to be understood comes to understanding for us. Here, then, the understanding achieved of a thing is not different from the way in which we can bring that thing to language, as an understanding for us 'thus-far'. Understanding is therefore the

\footnotetext{
${ }^{23}$ It is important to establish that this is not a solipsism. Hermeneutic Constructivism has suggested that the inextricable link between the prejudice, language and understanding means that a person can bring his or her understanding to language for another person. What is being suggested is that it would be unlikely for two people to have developed the same prejudices and even more unlikely that these same people have developed the same meaning. Importantly, this does not mean that two people cannot communicate with each other and share their view of the world while simultaneously apprehending another person's prejudices and their subsequent horizon. In fact, this reciprocity that is inherent within a dialogue operates to clearly and reflexively overcome solipsism.
} 
product of our engagement in the dialogue of languaging, as a to and fro movement between the horizon of the thing to be understood and the horizon that is provided by our prejudices.

Each thing has its own horizon. However, recalling the elaboration of the I-Thou relationship outlined previously, the thing itself cannot engage in a dialogue and instead must be made to speak. Here then, the dialogue that the thing is able to engage in is a function of the questions posed of it from the $I$, from us. In other words, the question posed determines the response that can meaningfully become an answer from the thing. For example, if one reaches toward the thing by way of the question 'Is that round?', there is clearly only a finite range of answers that could be meaningful responses. Thus the horizon of the thing can be considered as those answers that are meaningful possibilities to the question posed.

One will recall that each prejudice has a subsequent horizon that represents those aspects of authentic consciousness that a prejudice is able to provide access to. It is from within this horizon that the questions that sustain the ongoing to and fro movement of the dialogue of languaging are developed. It follows, therefore, that the horizon of each prejudice represents the horizon of possible questions that can be posed of the thing. Thus, when the horizon of the thing is able to answer the questions posed from the horizon of our prejudices, these horizons coalesce in a fusion of horizons. This fusion is a recognition that the questions posed of a thing are in fact legitimate, because they are asked in such a way that the horizon of the thing is able to provide suitable answers. If this were to be the case for a person, the prejudice from which that person reached toward this thing in understanding would be considered suitable for understanding this thing, given that it can open up towards further questions that are productive in the evolution of his or her prejudice(s).

The effectiveness of this anticipation is not to be confused with a complete or universal understanding in the form of an epistemological realism, or a one to one correspondence between a prejudice and thing. Instead, one must recall Gadamer's elucidation of the notion of application, where a thing to be understood can always be understood differently if considered from a different perspective. Thus, in the case of Hermeneutic Constructivism, a thing to be understood always has the possibility of being understood differently, if indeed it is envisioned from a different prejudice and its subsequent horizon. Here, then, a fusion of horizons is a recognition that the horizon of the prejudice is appropriate for envisioning the thing to be understood. Nonetheless, inherent in this position is recognition that another different vantage point is always available to envision that same thing differently. Thus, in light of the finite possibilities of human understanding and the fact that the moment a question is answered by the horizon of the thing further possibilities of questioning always arise, the fusion of horizons can never be complete. Reflexively, 
however, it is possible to understand something deeply. That is, the greater the extent of the fusion between the horizon of the thing and the horizon of our prejudices, the deeper our understanding is.

Apply - The Application Corollary has established that for a thing to be understood a person must apply his or her understanding 'thus-far' to the situation at hand. That is, we must apply a prejudice to an event of understanding and allow that event to be illuminated and to come to understanding from the vantage point of that particular prejudice. Importantly, however, this illumination does not take the form of a searchlight simply seeking something out in the form of a correspondence between prejudice and thing. Instead it is an illumination from within the event as a mode of being, an illumination from which all understanding has its origin and its possibilities and not its completion. Thus the thing that is to be understood is illuminated by the light of the prejudice and comes to be understood in terms of a thing for us, as a thing that is not different from the way in which it is illuminated.

It is, however, as previously noted, always possible for us to illuminate an event by the luminescent qualities of different prejudices and to understand that event differently as a function of the different illumination. Conceptualised in this way, understanding is not a perfection but rather always an application. It is the application of a prejudice to the situation at hand or an event of understanding, and it is that prejudice that determines the possibilities of the thing that can be understood. Again the notion of understanding as application carries with it an inherent productivity toward the ongoing development of a person's prejudices.

Horizon of That Which is Other - It has been emphasised previously that in order for us to bring a thing to understanding, that thing must come to language so as to be able to engage in the dialogue of languaging itself. As emphasised previously, the engagement in a dialogue by a thing does not represent a requirement for a metaphysical position that ultimately challenges the reflexivity of Hermeneutic Constructivism. Instead one will recall that it is recognition that everything in the world that is not Self, and is therefore other, must be made to speak or to engage in a dialogue - as outlined in the I-Thou relationship - in order to be understood. It was established above that each of our prejudices carries a horizon that opens up the possibility of what can therefore become a question in the evolution of our prejudices and therefore understanding. It is the questions asked of the thing that therefore provide that thing with its own horizon, a horizon of possible answers to these questions. Thus understanding always involves a coalescence of these horizons; the horizon of our prejudices, that provides the possibilities of what can become a question, and the horizon of the other as the possible answers to these questions. 
Fusion of Horizons - A genuine dialogue has been established as an ongoing to and fro movement that resembles the structure of questions and answer. It is this same essential structure that underpins languaging and the bringing of the world to understanding. This corollary sheds more light on the nature of this question and answer movement. Given that every question stands in need of an answer and every answer is ultimately a response to a question, then what is occurring is a coalescence of the horizons in languaging itself. The horizon of the prejudice which carries with it the possibilities of understanding, and therefore what can become a question, and the horizon of the other as the possible answers to that question create a fusion. That is, these horizons come together in a unity of agreement while always, and at the same time, have aspects of separation between these horizons. This separation represent aspects of disagreement and therefore the need for further questioning. Visualising the way in which a spinning coin slows down upon a bench-top captures this constant state of flux between horizons. As the momentum of the coin diminishes, it begins to rotate in such a way that when the left side is up, the right side is down and vice versa, until such a time as it stops on the bench's surface. The point at which the surface of coin meets the surface of the bench is representative of agreement, while at the same time the opposite end is not in contact with the bench representing aspects of disagreement and the possibility of further questioning. Most importantly, it is a dynamic movement that never comes to an end.

With the essential movement of this fusion considered, it is important to appreciate what this fusion actually means. Here in the dialogue of languaging we are able to recognise what it is that we already understand sufficiently about the thing, while at the same time becoming aware of what it is that remains to be understood. It is this recognition of what is in doubt that conditions the questions posed. Therefore, when we pose questions of the thing, these questions are ultimately questions of what we already understand. In other words, these questions are of our own prejudices. Thus, in a fusion of horizons, our prejudices and therefore our understanding, are developed through this coalescing of question and answer. The change in our prejudices that epitomises understanding is an achievement brought about always by a fusion of horizons.

\section{TEMPORAL DISTANCE COROLLARY - A PERSON IS ABLE TO CONTINUE THE LANGUAGING OF EVENTS FOLLOWING THE EVENT ITSELF.}

The Organisation Corollary emphasised that it is not possible for us to bring to understanding our prejudices in such a way as to distinguish in advance those prejudices that are productive, and therefore result in understanding, from prejudices that result in misunderstanding. The Temporal Distance Corollary provides an opportunity to refine this 
proposition. ${ }^{24}$ At the centre of the Organisation Corollary was a view that a person cannot be situated outside of his or her prejudice system in order make such determinations. However, in being reflexive to the fact that at times we do indeed change our opinions of what has gone on at a previous moment in time, the Temporal Distance Corollary suggests that it is possible for us to make discriminations about our prejudices.

The process of making discriminations about our prejudices requires engagement in a further dialogue of languaging. It is important to appreciate that it is not the event itself that a person is continuing to language. Instead what is being languaged is what we have kept of the event. That is, by making something of the event in the unfolding of the event itself, we developed our prejudice(s) and henceforth had an experience. Therefore, it is this experience that a person is now re-languaging in order to understand it differently. In this movement of languaging we can envisage that experience from the perspective of different prejudices and therefore continue to make something of it. It follows that if we make something of our experience, then we are actually developing further our prejudices and as a result are having a different experience. In so doing, we are able to filter prejudices that lead to adequate understanding from those that may lead to misunderstanding in the future.

Continue - This word emphasises that the development of understanding is not restricted to the time bound parameters of the original event of understanding. To 'continue' implies that something goes on from an initial position and that it has no end point, similar to our understanding of the world.

Following - After having made something of the event itself, we are able to continue to language those aspects that we have already made of the original event of understanding itself. Thus we are able to adopt the vantage points of our prejudices that may not have been applied in our understanding processes at the time of the event of understanding per se.

\section{Chapter Summary}

The Fundamental Postulate and eleven elaborative corollaries represent the core theoretical tenets of Hermeneutic Constructivism. At its core Hermeneutic Constructivism reflexively acknowledges a concomitant and inextricability of language, world, and person. It is anticipated that this foundation will provide an opportunity through which to catch a glimpse of the individual person going about the business of being human for the purpose of

\footnotetext{
${ }^{24}$ The title Temporal Distance Corollary borrows heavily from the work of Gadamer who suggested that the passage of time provides the person with a productive opportunity for filtering his or her prejudices. For a more complete review see Chapter Five.
} 
qualitative research. In light of the high level of abstraction with which Hermeneutic Constructivism has so far been considered, as well as the hopes held for the Hermeneutic Constructivist position more generally in redressing issues of representation for qualitative research, the following chapter provides a further elaboration of these complex abstractions. In addition, the following chapter will provide a consideration of the way in which Hermeneutic Constructivism can be considered to overcome the challenges previously levelled at the Psychology of Personal Constructs. 


\section{CHAPTER 7}

\section{HERMENEUTIC CONSTRUCTIVISM: AN ELABORATION}

The previous chapters outlined the theoretical basis of a position referred to as Hermeneutic Constructivism. This chapter is a descriptive elaboration of the Hermeneutic Constructivist position in order to provide greater comprehension of the theoretical tenets established in the previous chapter. A series of principles that inform good theory design as outlined by Kelly (1955) will be used as a framework with which to base a preliminary assessment of the Hermeneutic Constructivist position. This chapter will culminate with a discussion of the way this Hermeneutic Constructivist position addresses the hurdles identified of Personal Construct Psychology, this will be undertaken through the lens of Noaparast's (1995) notion of Sophisticated Realism. The following chapter will provide a consideration of the method underlying the application of the Hermeneutic Constructivist position for qualitative research.

\section{A Descriptive Elaboration}

The Fundamental Postulate and eleven elaborative corollaries that make up a consideration of how it is that a person goes about the business of being human for Hermeneutic Constructivism have at their core the notion of languaging. It has been elaborated previously that the dialogue of languaging conditions the way that a thing, that is to be understood, can in fact come to understanding. The to and fro movement of languaging itself is guided by a series of anticipatory and historically mediated structures - the prejudice. Although a brief and perhaps pragmatic sketch of the notions of languaging and the prejudice have already been provided, the centrality of these tenets to Hermeneutic Constructivism and its subsequent operationalisation warrants further consideration. It is important to note that although the prejudice is inextricable from languaging, in order to facilitate a closer examination of these concepts they are separated here under the titles the nature of languaging and the nature of the prejudice.

\section{The Nature of Languaging}

A theory that makes any claim to envisioning the way that a person goes about the business of being human - if indeed it is to be theoretically reflexive - must be unambiguous 
about the relationship between the processes of understanding, the thing that is to be understood, and language. It is for this reason that Hermeneutic Constructivism follows the work of Gadamer and his elucidation of language as the medium of being human. In adopting this position a decisive foundation is established that underpins Hermeneutic Constructivism and justifies a closer exploration of what is meant by the term languaging ${ }^{1}$.

Languaging embodies the movement of bringing a thing that is to be understood to understanding through the medium of language. The term 'movement' here is recognition that the process always begins from an initial position and is propelled towards a different position as the fundamental nature of the comportment of being. In other words, for Hermeneutic Constructivism, when a thing is to be understood, the process of understanding always begins from an initial understanding and moves to a different understanding through the dialogue of languaging itself. Importantly, however, this initial understanding is not an understanding that is somehow inherent within the thing itself. Instead, this initial understanding is a function of the prejudice from which we project towards the thing to be understood. Here we can recall the example of the first love. It was the initial prejudice of overwhelmingly positive emotion that the person projected toward the sensation to be understood. Here this sensation is not the emotion itself; in fact it is not anything that can be understood until we project an initial understanding towards it. It is then from this initial understanding that the movement of languaging begins. In relation to this example, the dialogical movement of languaging itself has its culmination in the prejudice this must be love.

At the core of languaging is the notion of a dialogue. Here a dialogue is a structure that does not have any predetermined end point. Instead, a dialogue, much like the structure of a conversation, follows a question and answer or a to and fro movement. This movement represents an inherent circularity where we can put something forward, as an initial understanding, and open it to questioning by another person. These questions, while perhaps settling some areas of understanding, always open up the possibility of further questions. Thus, what is actually going on in this dialogue is an ongoing determination of the degree of appropriation between our prejudices as well as their subsequent horizons and those of the person we are seeking to understand. Here appropriation is not simply an exercise of seeking agreement by another person with our vantage point or prejudices. Instead, in a genuine dialogue, this appropriation is recognition of the possibility of another person's prejudices,

\footnotetext{
${ }^{1}$ Maturana (1988) describes languaging in the following manner; "Whatever takes place in the praxis of living of the observer takes place as distinctions in language through languaging, and this is all that he or she can do as such" (p. 6i). Here Maturana is clear that language is the medium of being human.
} 
the possibility of another person's take on the world. It is this inherent openness toward the possibility of another person's view - the possibility of understanding differently - that is the comportment of our being, a comportment that is ultimately conditioned by the ongoing movement of languaging. This openness, therefore, not only sustains the possibilities of understanding one another in conversation, but also the possibility of understanding the world around us.

As described previously the dialogue of languaging between two people is easy to conceive of. However, when the dialogue of languaging is between a person and the world it is perhaps more difficult to apprehend. The I-Thou relationship as outlined by Gadamer (1960/2003), and as alluded to in the previous chapter, offers a valuable model. This model provides a foundation for apprehending even further the essential structure of languaging itself and the way that a dialogue between a person and the world can manifest. One will recall that in this relationship the $I$ embodies everything that we already have in terms of what we have made of the world in our previous ventures of understanding. The $I$ is that something that we have made of our previous here and nows. Thus the $I$ represents the understanding that we bring with us to an event of understanding in the present. The Thou, on the other hand, encompasses everything that is other, essentially everything that is not the Self. However, this Thou is not the things or these objects themselves, instead it is the way in which these things are related to the $I$. More specifically, a Thou is the way that these things are made to relate to the $I$. It is after all the $I$ or the Self that allows these things to enter the dialogue as a Thou.

Through this I-Thou relationship the centrality of question and answer can be emphasised. It is a question, that has its origins in the $I$, that ultimately allows the thing to become a Thou, and to provide an answer to that question. Here we are reminded again of the example used in the previous chapter of one's first love. The initial languaging centres on the idea that something, a sensation, was definitely happening that could not be fully understood. By projecting an initial understanding toward the sensations, the idea of an overwhelmingly positive emotion became the way that the sensation was understood. Through further languaging, and subsequent questioning of this initial understanding, an understanding became progressively refined. In fact, in the example, the understanding was refined to the point that the understanding that resulted was this must be love. This example suitably emphasises the centrality of question and answer to the notion of languaging as the way in which we ultimately come to understand the world.

This theoretical unpacking of the I-Thou relationship, that resembles the structure of languaging itself, provides a means of elaborating further the way in which languaging 
sustains our comportment toward understanding. That is, the movement of languaging is always toward understanding something better or differently. Therefore languaging has an inherent productivity that is always directed toward a better or different understanding through the development of our prejudices. As outlined previously and emphasised through the example of a first love, the questions that condition the I-Thou relationship have their origins in the $I$. That is, for a question to become a possibility means that something that we already have still remains open to being understood differently or better than the way it is at present. In the exemplar, the understanding of the sensations in terms of an overwhelmingly positive emotion, is possibly an adequate understanding that is sufficient to go on being with the world. However, in the dialogue of languaging, the person in this example recognises that the sensation or the emotion is more than that; it is more than any other positive emotion that he or she has experienced. It is at this point that the person asks questions of his or her initial understanding; questions that ultimately originate from the $I$, from what it is that the person already has.

In order to formulate a question the person must be able to make a determination of what is understood and yet still open to being understood better or differently. This itself involves a dialogue with the $I$, a dialogue with what he or she already knows in order to develop a meaningful question. Therefore the answers to these questions are developmental of the $I$. In other words, these answers close down some aspects of the question posed and yet always open up toward further questioning. It is here that the inherent productivity of the dialogue of languaging can be seen. That is, it is the $I$ that is affected, or changed as a result of the dialogue of question and answer. And as such the subsequent understanding that is projected from the $I$ becomes different to what it was before the question was posed. Thus, it is languaging that conditions the comportment toward understanding as an understanding better or differently.

\section{The Nature of the Prejudice}

Hermeneutic Constructivism reinstates Gadamer's (1960/2003) rehabilitation of the prejudice as an essential condition of understanding. As outlined in the previous chapter, a prejudice is a judgment that is made prior to all the available information being examined. In other words, it represents a tentative or initial understanding that is capable of being developed in the face of new evidence. A useful means of unpacking the nature of the prejudice is to liken it to the illuminating qualities of light. A prejudice illuminates an event and therefore allows it to be understood. In the absence of a prejudice, there is an absence of light and therefore there is no possibility of illuminating the world in understanding, or of discerning one aspect of the world from another. This inextricability of the prejudice from 
understanding, and therefore from the human comportment toward understanding, makes it a particularly high level of abstraction with which to be concerned. The prejudice therefore provides fertile ground for apprehending a high order understanding of the person for the purpose of qualitative research.

Having emphasised that the prejudice is an essential precondition of understanding, it is important to apprehend the way that the prejudice is inextricable from the movement of languaging itself. A prejudice is an initial understanding, it is that something that we already have about a thing to be understood prior to an event of understanding itself. Thus, the prejudice is an anticipatory structure that is established prior to its projection in understanding. In totality, then, our prejudices represent everything that we already have, that we already understand about the world. In light of this, the prejudice comes to represent the $I$ component of the I-Thou relationship. It is that understanding that we direct toward a thing to be understood and is ultimately the source from which the questions posed of that thing originate. It is important to appreciate that the prejudice does not first come to language and then become an $I$. Instead, from the outset, our prejudice is the Self, it is the $I$. In returning to the example, the initial understanding or prejudice of the sensation overwhelmingly positive emotion is the way that the sensation comes to be understood, it is the $I$ or that position from which the movement of languaging begins. Thus, in situating the prejudice as synonymous with the $I$ in the $I$-Thou relationship, the dialogue of languaging elaborated above in terms of developing a person's understanding, can now be unpacked further as the development of a person's prejudices.

\section{Authentic Consciousness and the Horizon of the Prejudice}

Central to an appreciation of the prejudice, as a fundamentally historical structure of understanding, the anticipatory nature of the prejudice stands in need of further consideration. Being synonymous with the $I$ in the $I$-Thou relationship, a prejudice represents an initial understanding that is projected towards the world and that enables the world to come to understanding. Here the words of T. S. Eliot (1976) provide a useful entrée to a discussion concerning the inherent historicity in the prejudice itself. That is, the way in which "timepresent and time-past are both perhaps present in time future, and time future contained in time-past" (p. 13). A prejudice is the embodiment of time-past as that something that we have made of the world in prior ventures of understanding. Given that it is this same prejudice that we ultimately come to project towards the world in understanding in timepresent, we can only ever come to know time-present in the light provided by the prejudice; a light that is ultimately that something we have made of time-past. Here the essentially 
historical nature of the prejudice becomes palpable, a historicity that can be emphasised further through a consideration of the notion of authentic consciousness.

The notion of authentic consciousness allows a closer examination of the way that a prejudice is able to provide access to everything that we already have about a thing to be understood. In following Gadamer's (1960/2003) elucidation of the speculative qualities of language, the prejudice as an initial understanding that is projected towards a thing to be understood, always opens up toward further meaning. That is, the prejudice fills itself with meaning that is more than simply what the prejudice itself says. The example of a first love is again useful. The initial prejudice overwhelmingly positive emotion is the way in which the sensation is understood, and opens up toward the dialogue of languaging. In that dialogue other aspects of what is known about the thing come into the dialogue of question and answer as opportunities to develop this initial prejudice and to open up further questions. For example, is this positive emotion the same emotion as winning a football final, or is it the same feeling as riding my bicycle down a hill for the first time. These examples are but two of an infinite array of positive emotions that the prejudice, overwhelmingly positive emotion, might fill itself with, and allow those aspects of what the person has made of his or her times past to come into the dialogue of languaging in time-present. This capacity to fill itself with meaning, beyond what is explicitly stated by the prejudice, and to bring all that we already have about a thing to be understood into the dialogue of languaging is a function of authentic consciousness.

Authentic consciousness is the totality of what we already have of the thing to be understood and that is able to come to the dialogue of languaging by way of a specific prejudice(s). Here, then, the term 'authentic' is used to indicate a consciousness that already been affected or influenced by that something that a person has already made of previous events of understanding. It is a consciousness that has therefore become itself, become a consciousness in terms of an I or the Self. In other words, authentic consciousness is the totality of what is already available or what we can bring to the dialogue of languaging. It is important to appreciate, however, that authentic consciousness is not a special consciousness or a position from which a person can survey the entirety of his or her historicity. Instead, a person only comes to know those aspects of authentic consciousness that a specific prejudice provides access to. That is, the prejudice projected in understanding is the same prejudice that provides entrée to the dialogue of languaging. Thus it is the inextricable connection between the prejudice and authentic consciousness that conditions and sustains the dialogue of languaging itself. 
As outlined above, the questions that are asked of the thing to be understood have their origins in the $I$ and involve an appreciation of what it is that is understood, as well as of those aspects of the thing that could be understood better or differently. In fact, it is this inclination toward the development of our prejudices as the development of the $I$ that represents the comportment of being. It is, however, authentic consciousness that informs the dialogue of the $I$, and the development of an appropriate question that will facilitate the development of our prejudices. To return to the example of a first love, the initial prejudice overwhelmingly positive emotion opens up toward aspects of authentic consciousness in the manner outlined above. Here this essentially inner dialogue between the thing, or in this case the emotion, and the $I$ as authentic consciousness, might allow a question in the form of "is it like winning the football final?' to originate. This question would be filled out by what is already understood of that experience in terms of its qualities as a positive emotion from authentic consciousness. Recall, however, that this filling out with meaning always opens up further possibilities for ongoing questions on the basis of what is now understood of the emotion. In fact, in the ongoing dialogue of languaging, those aspects of authentic consciousness that can be accessed can both expand and contract as a function of the questions that are being asked and the prejudice(s) that come to the dialogue of languaging. Thus, the prejudice as the essential conduit to authentic consciousness, emphasises the historicity of the prejudice itself and situates the person of Hermeneutic Constructivism as a fundamentally historical being.

Emphasising the historical nature of the prejudice, the Horizon Corollary suggests that each prejudice carries with it a horizon or vantage point from within which a thing to be understood is able to come to understanding. The horizon that is perhaps most familiar is the horizon formed by a unity between the earth's surface and the sky. This point represents the limit of what can be seen of the earth from a particular vantage point. In terms of a person's prejudices, the horizon represents the limits and therefore the possibilities of what can be understood from the particular vantage point of that prejudice. In relation to authentic consciousness, then, the horizon of the prejudice represents that aspect of authentic consciousness that is accessed by that specific prejudice. In order to unpack further the notion of the horizon of a prejudice, a more detailed consideration of what is meant by the antithetic nature of the prejudice is useful.

\section{The Antithetic Nature of the Limits of the Prejudice}

Underpinning the view that it is not possible for the person that Hermeneutic Constructivism envisages to develop a single prejudice that enables the world to come to complete understanding is recognition that the horizon of each individual prejudice has limits. 
That is, the vantage point of each horizon is limited with regard to what can meaningfully be understood from the position that it provides. Thus, much like the earth's horizon, there is always something that lies beyond these limits, a something that cannot be envisioned from that vantage point. As outlined through the Antithetic Corollary, then, the prejudice itself has what has been termed a context. A context represents the outer limits of the prejudice or the point at which the thing that we project our prejudices toward in understanding can no longer be meaningfully understood by way of that prejudice. ${ }^{2}$ The prejudice itself carries that which is ready at hand, or that which is evident and is referred to here as the Evident Prejudice. However, this prejudice also carries the possibility of another position that, although less obvious, is implicit in the structure of the prejudice itself - the Antithetic Prejudice. The nature of this later prejudice has its origins in the work of Hegel (1807/1967) and Kelly (1955) in the manner previously outlined. ${ }^{3}$ As suggested previously, however, the antithesis represents not only the opposite of the Evident prejudice itself, but in combination the Evident and Antithetic prejudices represent the context of the prejudice. The context can be considered therefore as that spectrum of possible understandings that are situated between the Evident prejudice and the Antithetic prejudice.

As well as providing the context of a prejudice itself, the evident and antithetic nature implicit within the prejudice itself is also suggestive of the outer limits of the horizon of a specific prejudice. That is, the context of the prejudice determines those aspects of authentic consciousness that can be accessed in the dialogue of languaging. Recall that a horizon embodies the possibilities available for envisaging the world from the vantage point of a particular prejudice. Thus, in terms of the context of a prejudice, the Antithetic prejudice represents the point at which the possibilities of understanding or the illuminating capacities of the prejudice become dim. It is important to appreciate that the Antithetic prejudice represents that point beyond which the world cannot be illuminated in understanding via that prejudice. Thus, the antithetic prejudice itself is the outer limits of the horizon (for that prejudice) from which it is still possible to illuminate the world albeit dimly.

\section{The Influence of Experience on the Nature of the Prejudice}

In remaining consistent with the conceptualisation of the proactive person that Hermeneutic Constructivism envisages, it is important to apprehend the way in which

\footnotetext{
${ }^{2}$ Importantly for Hermeneutic Constructivism, this antithesis does not come, as such, it arrives. The use of the word arrive here emphasises that an antithesis is not something that is necessarily ready at hand. Instead, a person asked to provide an antithesis to a prejudice would normally need to engage in an internal dialogue or languaging in order to arrive at the antithesis itself. Thus, an antithesis, like the prejudice, has its being in language and arrives as a product of the dialogue of languaging.

${ }^{3}$ For a review of the work of Hegel (1807/1967) and Kelly (1955) see Chapter Six and the elaboration of the 'Antithetic Corollary'.
} 
experience informs both the context and horizon of a prejudice. Recall that experience is that something which is made of the event of understanding. In other words, experience represents a change or development in a person's prejudice(s). In light of the more refined consideration of the nature of the prejudice, it is possible to now be more specific about what form this change in a person's prejudices may take.

Through the dialogue of languaging, both the Evident and Antithetic prejudices develop in such a way as to provide a better or different understanding. This might involve a refinement of those aspects of the world that a prejudice(s) can be considered useful for bringing meaning to. Such an evolution would therefore represent a refinement in the context of a prejudice. This evolution in a prejudice's context equates to a subsequent evolution in the horizon of that prejudice, and therefore a change to the point beyond which the world can no longer be illuminated in understanding by that prejudice. For example, it would not be unreasonable for a person to identify 'death' as the antithetical parameter of the horizon of the prejudice 'life'. However, a medical physician, having experienced on potentially numerous occasions the varying acuteness of illness prior to death, may in fact identify 'rather unwell' as the antithetical parameter to the prejudice 'life'. Here it becomes clear that experience is the sustenance of authentic consciousness and so to the antithetical limit of the horizon of a specific prejudice.

\section{The Illuminating Nature of the Prejudice}

The notion of a horizon of the prejudice emphasises that a person can adopt a vantage point anywhere along the spectrum formed by the Evident and Antithetic prejudices in seeking to illuminate the thing to be understood understandingly. However, one must recall that no single prejudice is sufficient to illuminate every event of understanding toward which that prejudice is projected. It is for this reason that each person develops a series of prejudices in order to bring the world to understanding. It is important to recall, however, that the prejudice does not represent an instrument for a surreptitious epistemological realism that seeks a correspondence between the prejudice and the thing to be understood. Instead, the prejudice represents a beginning point for understanding. So much so, that the illumination qualities of a prejudice found to be inadequate only becomes apparent in the light provided by a different prejudice. The example of a first love is explanatory of this. The initial prejudice, overwhelmingly positive emotion provided a suitable beginning point for entrée to the dialogue of languaging. Through a question and answer movement, this person reaches the prejudice this must be love. It is from the vantage point of this prejudice, that the initial prejudice - overwhelmingly positive emotion - can be seen as less representative, and would therefore be open to the possibilities of evolution and or context refinement. 
Although all understanding has its origins in the illumination qualities of a prejudice, it is important to recall as per the Passive Corollary that a person is not engaged in the dialogue of languaging a prejudice at every moment in time. Instead, a person can actively chooses to be inactive with regard to his or her languaging efforts. In remaining reflexive to the everyday business of being human, there are many events of understanding that our prejudices are capable of sufficiently bringing to understanding in such a way as to go on being. In other words, even though our understanding always has the possibility of being better or different, many of our prejudices can and do provide a satisfactory or sufficient understanding of the thing to be understood in order for us to go on being with the world. To appreciate what is meant by this satisfactory or sufficient nature of a prejudice Gadamer's (1960/2003) notion of understanding as interpretation is re-visited.

\section{The Interpretive Nature of the Prejudice}

As established by Gadamer (1960/2003), and rehabilitated here, all understanding is interpretation. Interpretation is not an impediment to or a limitation of human understanding, as the arbitrary assignment of meaning to something else. Instead understanding is always interpretation, to the extent that interpretation is the explicit form of understanding. Gadamer's (1960/2003) discussion of translation from one language into another provides a useful means of apprehending what understanding as interpretation means. Gadamer (1960/2003) suggests that in translation a person makes judgements about what is to be translated. The person finds the words that are appropriate to carry the meaning of the text that is being translated, while acknowledging that this translation will also succumb to a loss of some of the overtones and vibrations that are in the original. The point to be emphasised here is that in any interpretation accent is placed upon those elements that allow the thing to be interpreted and to come to understanding.

Interpretation is, then, a consideration of how well a prejudice is able to accent aspects of the thing that is to be understood. As per the Application Corollary, a person applies or projects a prejudice towards the world and allows the world to come to understanding from the vantage point it provides. In applying that prejudice it illuminates or accents aspects of the world and ultimately provides an understanding of that event. The adequateness of this accenting or illumination is one of degrees. ${ }^{4}$ A poor or less adequate

\footnotetext{
${ }^{4}$ The use of the term adequateness is not to be confused as a slippage into an epistemological realism where a person simply seeks a correspondence between his or her prejudices and the thing that is to be understood. Instead, one must recall that we always illuminate the thing that is to be understood from within the event of understanding itself. So much so, that a position that is independent of this illumination, a position that would be required to make such comparisons is impossible to establish. Instead the term adequately recognises that the comportment of being toward understanding is
} 
interpretation only poorly accents or illuminates the thing to be understood, while a good or more adequate interpretation allows the thing to be understood or for the representation to be effaced by it. That is, where the prejudice or interpretation illuminates the thing to be understood adequately, the interpretation disappears as an interpretation at all. Thus, where the prejudice that we project toward the world in understanding is adequate, it allows that thing to be understood to come to understanding. That is, in an instance where the prejudice is adequate, the prejudice itself is effaced as being simply a pre-judgment and instead is the thing for us. Here the person does not need to engage in the dialogue of languaging in order to move toward a different prejudice in an effort to bring that thing to understanding. Instead, this initial prejudice simply persists, and therefore nothing extra is made of the event towards which that person projected that prejudice(s). ${ }^{5}$ Rather, that event simply passes as a timepresent into a time-past, given that the prejudice was sufficient in order for that person to go on being in the world. Returning to the nature of understanding as being adequately sufficient, one can now apprehend more completely that understanding is never an understanding of a 'thing in itself' but rather always an understanding of the 'thing of itself'. Understanding is therefore only ever an understanding of those aspects of the thing that are sufficiently accented by our prejudices, and that, therefore, allow that thing to be meaningfully understood. ${ }^{6}$

The Temporal Distance Corollary takes the notion of the adequateness of a prejudice even further. This corollary recognises that each of us always has the capacity of reflecting upon our experiences and of continuing the dialogue of languaging after the event of understanding itself. This provides the person envisaged by Hermeneutic Constructivism a capacity to filter those prejudices that provide an adequate understanding from those that do not. As outlined above, however, we cannot merely reflect on the entirety of our prejudice system in order to make these determinations. Instead we re-envision an experience from the vantage point of different prejudices in the dialogue of languaging itself. In other words, we can illuminate an experience by the light of different prejudices, and in doing so determine if different aspects of it can be accented and therefore understood better or in a different way

ultimately towards an understanding that is adequate in order to go on with the world. This position supports the idea that a thing can always be understood from a different vantage point within the horizon of a prejudice and that a person can always apply a different prejudice to understanding that same thing in a different way.

${ }^{5}$ On the other hand, a prejudice that only provides a less appropriate illumination compels a person toward engaging in the dialogue of languaging in order to develop his or her prejudice system. Gadamer (1960/2003) said that, "every experience worthy of the name thwarts an expectation" (p. 356). To paraphrase Gadamer in terms of what experience is for Hermeneutic Constructivism, genuine experience 'thwarts an anticipation'.

${ }^{6}$ Gadamer (1960/2003) considers the notion of understanding in Hegelian terms, "the 'in-itself' is a 'for-me"' (p. 466). 
after the event itself. This filtering process therefore provides a person with the ability to project a prejudice in such a way that he or she more adequately illuminates an event of understanding in the future.

\section{The Prejudice and Qualia}

It has been established that the comportment of being human is always toward understanding. Given that all understanding occurs entirely in the light provided by one or more prejudices, it is the prejudice itself that comes to represent the most fundamental ontological structure of being for Hermeneutic Constructivism. The prejudice is therefore the position from which all understanding of a thing to be understood originates. This is no exception when the things are your qualia. Qualia are the qualities of our internal experience. In order to apprehend the inextricable connection between our prejudice(s) and our qualia, it is important to first appreciate what is implicit within the notion of something having qualities.

A quality is a distinctive attribute or characteristic of something. For a thing to be attributed the status of having a quality it must be understood in some way. That is, the thing must be brought to language, and the dialogue of languaging, in order that its quality(s) can be determined. It follows, therefore, that in order for that thing to have entrée to the dialogue of languaging and for it to come to be understood, a person must apply a prejudice to it. The fact that qualia are considered to be essentially inner processes does not mean that they conjure a different mode of understanding. Instead our innermost sensations, our feelings, our emotions are merely things that stand in need of being understood, even if only by us, and therefore must come to the dialogue of languaging in order to be understood. Here an exercise is perhaps useful.

Without seeking to be trite, 'just be', that is, 'just sit' and do not offer a response to the following question instantly, rather, wait several seconds. Now, the question 'How are you feeling?'

The purpose of the exercise is to highlight that emotions, feelings and thoughts are indeed consubstantial with words. If you have conducted the exercise effectively, you will notice that until a response is provided to the question, the feeling itself does not become anything. In fact, even if you do not know how you are feeling or you are feeling nothing then you are in actual fact feeling something. You are feeling what it is that has come to language. It may well be nothing. Thus, the way in which feelings are brought to language is not different from the way that these feelings are for you. This is not a correspondence of your prejudice with a thing that has some predetermined meaning. Instead the way that you bring that feeling to 
language, by way of what you already know in the form of your prejudice, is the thing of itself; the thing as it is for you. Thus, qualia are not different from the way in which they come to language as a prejudice.

\section{The Nature of the Business of Being Human}

Adopting the view that the prejudice represents the highest order structure that can be abstracted from the person that Hermeneutic Constructivism is concerned with, presupposes some inherently different theoretical accounts of aspects that are traditionally fundamental to the business of being human. In order to provide a foundation for a beginning appreciation of the fundamental tenets of the Hermeneutic Constructivist position, the world was set down in terms of that stream of events towards which we actively direct our understanding processes. Here the focus is on situating the person that has been elaborated through the theoretical unpacking of the Fundamental Postulate with this world. The way that this person is able to share his or her view of the world with other people will also be considered. This consideration begins with a look at the person.

\section{The Person}

It is important that any theoretical position that has chosen the person as the focus of consideration makes clear the level of abstraction with which this consideration will be made. In other words, is that person considered to be the same as every other person, or is this person in some way different from all other people? Hermeneutic Constructivism is explicit that its major consideration first and foremost is with the individual person and not with groups of people. At the core of this consideration is a view that what makes a person a unique entity in itself is the notion of difference.

At the core of the Fundamental Postulate is an emphasis upon the way that each person develops a series of highly nuanced prejudices from which the world comes to be understood. There can be little doubt that each person has actively engaged in an immeasurable and intensely personal range of events of understanding. Those somethings that the person makes of each of these events determines his or her authentic consciousness and therefore the possibilities of what can then come to understanding in the future. Thus the idiosyncratic nature of each person's prejudice system, and the subsequent connections that these prejudices make in the authentic consciousness of that person, operate to make each of us inherently different from other people. Thus, for Hermeneutic Constructivism, it is difference that is at the core of what it is to be a person. This difference is not a special mode of contemplating the person; it is the mode of being a person. It is this same difference that is considered to be representative of the business of being human and the reason why 
Hermeneutic Constructivism is seeking to uncover these fundamentally idiographic aspects of being a person for qualitative research.

It has been outlined previously that the person Hermeneutic Constructivism is concerned with is compelled to adopt a certain attitude or comportment towards the world. This comportment is always towards an understanding that is adequate to go on being in the world. To apprehend this idea further, we must recall the theoretical unpacking of the prejudice as providing a source of light by way of which the world is illuminated. The prejudice is the pre-understanding that a person projects towards the world in understanding. Although a single prejudice cannot illuminate in understanding each aspect of the world, in their absence, or in the absence of their light, we cannot begin to discern one aspect from another in understanding. Hence a prejudice illuminates an event of understanding from within that event and, as outlined above, adequately accents aspects of that event while at the same time less adequately accents other aspects of that same event.

At the core of the comportment toward understanding is the development of our prejudice(s). Although the tendency toward developing a prejudice's capacity to illuminate those aspects of the world that are less adequately accented would seem most consistent with a comportment toward understanding, this is not always the case. Instead, remaining reflexive of the human situation, many people continue to illuminate the world in the same way, even in the face of contradictory evidence. At first it may seem that such a person has not developed his or her prejudices. On the contrary, however, that person's prejudices have developed, just in a different way. Often people who continue to illuminate the world by the light of the same prejudices develop that prejudice's capacity to illuminate more brightly those aspects of the world that are already accented by the prejudice. Here a belief in God provides a suitable example.

A zealot, for example, would seek to illuminate brightly those aspects of the world that corroborate his or her beliefs, and to cast in shadow any aspects that challenge that view. Importantly, although the zealot may dim the light that he or she shines on these contradictory aspects of the world, these aspects do not cease to exist. To recognise a need for the light of a prejudice to be re-focused or redirected means that the zealot must first become aware that his or her prejudice(s) are in fact capable of illuminating these contradictory aspects, and that they are therefore a possibility of understanding. However, by focusing more narrowly the light of his or her prejudice, the zealot is able to adequately go on being, all the while denying the possibility of such a contrasting and at times contradictory view. In other words, a person can seek to hold onto a view of the world, and to strengthen that view by focusing or 
narrowing the focal point of the light provided by a prejudice in the ongoing comportment of being human; of being a person.

\section{The Person and World}

Situating the comportment of being towards understanding better or differently, and therefore of developing our prejudices, means that the world and the person are inextricably linked. To apprehend the nature of this link more clearly we must appreciate the directionality of this relationship. Although the world has been described as being in a constant state of movement, of happening, this is not to be taken as a constitutive prompting into action of the person by the world. On the contrary, the world only comes to be understood by the proactivity of the person. More specifically by way of the prejudice(s) that the person projects toward the world in understanding and the subsequent dialogue of languaging that the prejudice(s) provide entrée to. In light of this, the comportment towards understanding compels the person to project a prejudice in order to bring the world to understanding. This comportment toward understanding is therefore the condition of being in the world.

The bringing of the world to understanding in the dialogue of languaging is, however, not to be confused with a simple idealism, where we simply bring the world to understanding in any way that we see as adequate. Instead the dialogue of languaging and the resultant understanding of the world is always guided by our greater system of prejudices. The Organisation Corollary established that our prejudices and greater system of prejudices are determined by an inherent tendency towards lawfulness. Given that the notion of lawfulness will be discussed in more detail later this chapter, it is enough to say at this point that the prejudice(s) that are evanesced toward in the ongoing movement of languaging are contingent upon those aspects of authentic consciousness - our historicity - that are opened up in the dialogue of languaging.

\section{Person with Person}

As the fundamental ontological structure of the comportment of being, the prejudice represents the highest level of abstraction with which the person envisaged by Hermeneutic Constructivism can be understood. Moreover, the theoretical unpacking of the Hermeneutic Constructivist position has established that the comportment toward understanding is inextricable from language. As a function of this inextricability the prejudice also has its being in language. Thus, given that the prejudice itself is not different from the way that it comes to language, we are able to be articulate about our prejudices with not only our self but also with other people. 
Despite its focus upon the idiographic nature of being human, the Hermeneutic Constructivist position is reflexive to the presence of other equally active languaging people within the world. The Sociality Corollary has established that a person is able to have a social relationship while maintaining his or her inherently idiographic view of the world. Here a social relationship does not require that we simply adopt the view of the other person as our own in order to form a relationship. Instead, in order to have a social relationship, we need to simply recognise another person's vantage point as merely one possible position from which to envision the world. Thus, at its core, a social relationship involves one person sharing aspects of his or her horizon or vantage point with another person.

Examples of this sharing of horizons are common in everyday interactions. Here, however, we do not simply adopt the position or vantage point held by another person as a mere presence in a monologue with that person. Instead, we always envisage the possibility of the other person's perspective from the vantage point of our own system of prejudices. It is true that we may indeed develop our prejudices as a function of this dialogue but this change alone is not a pre-condition of a social relationship. For example, a dialogue between two people concerning the merits of 'Football Team A' over 'Football Team B' is unlikely to change the opinion of an avid supporter of 'Football Team B', even though there is little doubt that this is indeed an example of social relationship.

A social relationship is inherently dialogical in nature. One person brings an aspect of his or her horizon to language and the other person allows that position to engage with aspects of his or her authentic consciousness. In so doing, this person allows the position of the first person to come to understanding. In other words, aspects of another person's horizon are always made sense of from the vantage point of our own. Thus, in order to have a genuine social relationship, we must engage in the dialogue of languaging. It is important to appreciate what is meant here by the use of the term 'genuine'. It is easy to recall instances where we have engaged in a social enterprise that was not genuine. In this instance, at least one person within the exchange is either not interested, or unprepared, to allow the horizon of another person to engage with his or her own horizon. Such a non-genuine social relationship might include a discussion with a repeatedly delayed tradesman delivering yet another excuse, or an abusive husband making demands of his wife while she asks him to leave the house. Here a person certainly engages in a dialogue, and yet is not engaged in a social relationship per se. A genuine social relationship thus embodies reciprocity between the interlocutors as a sharing and coalescence of horizons.

The discussion thus far has focused upon disclosing what is meant by the notion of a social relationship between people engaged in a dialogue. Despite this, it is important to 
apprehend that the reciprocity that goes on between interlocutors is one of degrees. That is, in keeping with the tenets of the Passive Corollary, each of us has the capacity to be actively passive regarding the reciprocity required of a social relationship. There are many occasions where interaction occurs between people that is completely non-verbal. For example, a bump, or a wink, a thumbs-up or a 'knowing' glance. In line with this, a look at an example of three people, in a lift, in a multi-story building is valuable.

One person greets the other person in the lift upon getting in. At the next floor, the door opens and on gets a rather eccentric character. The original occupants of the lift look at each other, one raising his eyebrows and the other grits her teeth. At the next floor the door opens and one of the original occupants exits. The non-verbal cues that went on certainly represent, at some level, a reciprocity on the part of these two people, that might reasonably represent an acknowledgment of that third person's eccentricity. It would be silly to suggest that is not a social relationship at some level. It has already been established that in order for any aspect of human interaction - for that matter any aspect of being human - to be understood, it must be brought to language. ${ }^{7}$ In other words, in order to understand these actions as an interaction they must be brought to language by each of the people in the lift for him or her self. Even if it is only to language a view that the other person agrees, that something was not right with the third person in the lift, a reciprocity has indeed occurred. Albeit this is a reciprocity that will only lead to a superficial or cursory understanding of this other person. In line with this it must be said that outside of an engagement in a dialogue about that experience at a later stage, neither occupant will ever come to understand, more deeply, what the other person really meant by his or her actions.

In the context of a social relationship, however, it is important to recognise that the bringing of these actions to language alone is not sufficient to sustain a social relationship. Instead what is required is again reciprocity on the part of the interlocutors. This requires that one person bring into the dialogue an aspect of his or her horizon that will provide a means of establishing a context and in so doing allow the action itself to be understood more sufficiently. For example, a person who bumps the shoulder of another person and quickly turns to say 'Oh...I do apologise...that was an accident' provides a context to the action, that the action alone does not. This context, as a sharing of an aspect of a person's horizon,

\footnotetext{
${ }^{7}$ Here one may recall Gadamer's (1960/2003) statement that "being that can be understood is language" (p. 474). This statement operates to solidify language's place as the medium of being human. Hermeneutic Constructivism, as has been stated previously, takes this assumption elaborated by Gadamer as entirely correct, such that for Hermeneutic Constructivism language is the medium of the business of going about being human.
} 
allows the person bumped into to apply a prejudice to that event of understanding in a way that leads to a more adequate understanding of what another person actually meant.

Within this coalescence of horizons it is important to appreciate more specifically those aspects of a person's authentic consciousness that can be brought to language. That is, a consideration of the degree to which a person is able to be articulate about his or her feelings, beliefs, and values, as well experience, for another person. It has been established that the prejudice is inextricable from language and that each prejudice provides a conduit to an aspect of authentic consciousness that informs the ongoing dialogue of languaging. Moreover, the prejudice itself as the condition of the human comportment is also the essential precondition of qualia. So much so, that for Hermeneutic Constructivism there is no separation between the quality of an internal experience or experience itself and language, as emphasised by the exercise outlined above. With this in mind a person can indeed be articulate about his or her inner aspects of being. However, this articulateness is not to be confused with a reflecting upon authentic consciousness and simply describing it. Instead, this articulateness is limited to those aspects that come to the dialogue of languaging itself by way of the movement of question and answer. It is important to appreciate that this limitation in the degree to which we can be articulate about our inner aspects of being, is not a limitation upon human understanding. Instead it is a limitation only of the questions that can be asked. Thus, by questioning and questioning further in the dialogue of languaging, a person can articulate the way that the world is for them.

Given the inextricability of the essential structures of understanding and language, the question still to be disclosed is: does a person mean what he or she brings to language about him or her self for another person? In remaining consistent with the proactivity of the person of Hermeneutic Constructivism, the answer to this question is a resounding 'yes'. However, as disclosed in the Passivity Corollary, this person is equally capable of being actively inactive with regard to the aspects of the world that he or she chooses to engage in the dialogue o languaging. In terms of the person with person relationship being considered here, then, this person can be actively inactive with regard to those aspects of him or her self that are disclosed. For example, it would not be unreasonable for a person to outline a dislike for a manager with a neighbour and yet be very guarded about sharing such details with fellow work colleagues. Here the person means exactly what he or she has brought to language, although it may not be what one wanted or expected to hear. 


\section{The Mot Juste and the Carriage of Meaning}

The unpacking of the Fundamental Postulate has emphasised that the comportment of being is always towards understanding. Also established previously, the dialogue of languaging is conditioned by the prejudice that is projected towards the world in understanding. That is, the light provided by the prejudice illuminates the event of understanding itself, and allows it to come to understanding. Given that it is the prejudice that provides entrée to the dialogue of languaging, the comportment toward understanding is ultimately an understanding of the world as it is for that person; specifically an understanding thus far. That is, an understanding as an interpretation. Having explored some of the key theoretical aspects of Hermeneutic Constructivism the nature of interpretation can now be refined further.

At the core of the comportment toward understanding is the ongoing development of our prejudices in order to more adequately illuminate an event of understanding. One will recall that the illuminative qualities of the prejudice are contingent upon those aspects of authentic consciousness that the prejudice itself provides access to. In another way, it is authentic consciousness that informs and sustains the ongoing dialogue of languaging and therefore the capacities of the prejudice to illuminate the thing that is to be understood. Thus, at the core of the comportment of being toward understanding, is a search for the most adequate prejudice, or that prejudice that provides access to those aspects of authentic consciousness that can sustain the ongoing dialogue of languaging. It can be said that this comportment is therefore always toward the most perfect word or phrase as the most perfect prejudice, to allow a thing to be understood. This is, as Gadamer (1985), cited in Grondin (2003), described it, the Mot Juste.

One will recall that Gadamer described the search for the Mot Juste as an unsatisfying search. ${ }^{8}$ The unsatisfying aspect of this search is that even when we find the most perfect word or phrase to illuminate the thing, we always know that at the same time we have not completely found it. Thus, for Hermeneutic Constructivism, the Mot Juste is the term given to the prejudice that most adequately embodies the event of understanding toward which it is projected. That is, it accentuates aspects of that thing so adequately that it allows that thing to be understood in such way that the prejudice is effaced as being a prejudgment. Here, in relation to the first love example, the initial prejudice of an overwhelmingly positive emotion provided a suitable entrée to the dialogue of languaging, and yet did not adequately capture the sensations that where happening. Through the dialogue of languaging, the prejudice this

\footnotetext{
${ }^{8}$ For a review of Gadamer's discussion concerning the Mot Juste see Chapter Five 'The Disclosure of the World in Language'.
} 
must be love was reached and while it is at that moment in time the Mot Juste, and it may adequately represents a person's internal experience, that person always has the possibility of recognising that in fact he or she has not completely found it.

Although it is the search for the Mot Juste that epitomises the comportment of being towards understanding for each person, it is the same search that goes on in a dialogue between people. Here one seeks the most perfect word or phrase to allow him or her self to be understood by another person. It is important to appreciate that this is not a process of mere reflection upon, but the product of the dialogue of languaging itself. In other words, the Mot Juste represents the most perfect prejudice that provides access to those aspects of authentic consciousness that are able to provide entrée to a dialogue with another person. This perfection is such that the Mot Juste embodies most perfectly our understanding of a thing, in such a way that that perfection can reach the other with a preservation of meaning. Here a suitable example is the communication that goes on between a parent and a child developing language. For example, trying to explain the use of the phrase I did not mean literally to a six-year-old child. In the dialogue of bringing this phrase to understanding we seek suitable examples to accentuate different aspects of it and gain a tremendous sense of joy when the child finds the Mot Juste or the phrase that highlights a carriage of meaning.

Despite the fact that the Mot Juste epitomises the comportment of being, it is important to appreciate that not everyone achieves the Mot Juste in every situation. In other words, not every prejudice allows an event of understanding to be adequately understood, and is therefore in need of being engaged further in the dialogue of languaging. In addition, we have all borne witness to people we have engaged in a dialogue who state that, "I just can't put it into words" when talking about an experience or an emotion. Here this may indicate that that person has not yet found the Mot Juste that would best allow the carriage of meaning to another individual. To continue with the example of a first love, it may be that the person in the example is in the process of trying to understand a series of intrusive and overwhelming emotions that are presently being understood as an overwhelmingly positive emotion.

Although this individual recognises that this is indeed not the Mot Juste, he or she cannot find a better word or phrase to bring it to understanding differently. That is, this prejudice does not capture effectively what these sensations actually are, thus far, for that person. Although the recognition that a person cannot find the Mot Juste in order to support the carriage of meaning of his or her qualia to another person may in fact indicate a genuine inability for a person to locate the Mot Juste, it may also indicate an unpreparedness or avoidance of engaging in a search for it. 


\section{Transcending the Obvious}

It follows that a person seeking to develop a new theoretical position is in some way dissatisfied with what existing theory offers. Here that dissatisfaction is with contemporary approaches to qualitative research that have been shown to inadequately represent the experiences of the inherently idiosyncratic people studied. At the core of these representational challenges is a belief that the uncovering of what is common between people ultimately culminates in an uncovering of what is obvious. It is for this reason that a statement made by Kelly (1969) regarding the desire to 'transcend the obvious' could be considered the motto of Hermeneutic Constructivism.

It is the goal of Hermeneutic Constructivism to outline a theoretical position that enables the focused study of an individual person at the level of the structures that condition his or her internal experience. It is anticipated that an exploration of a person at this level of abstraction will provide a theoretical platform for a deeper, more nuanced and therefore less obvious representation of being human for the purpose of qualitative research. If what is uncovered in the eventual application of Hermeneutic Constructivism is itself one day deemed obvious, then it is hoped that it will have constituted a theoretical platform from which to dive to greater depths in the search for a better understanding of human experience. However, in order that Hermeneutic Constructivism can be made redundant, it must have first opened up a dialogue about issues that concern the representation of real people and real lives within qualitative research. If nothing more comes of Hermeneutic Constructivism than the opening up of this dialogue and its subsequent relegation to the annals of time-past, then it will have served its purpose. That is, it will have stimulated, at some level, qualitative researchers to contemplate the development of theoretical possibilities that open up opportunities to explore human experience in a manner that ultimately transcends the obvious.

\section{Interlude}

The theoretical unpacking of the Hermeneutic Constructivist position could so far be challenged for being simply a 'best of' or 'cherry picking' approach to theory development. The selection of specific aspects of existing theory, whilst neglecting other aspects of the same theory, represents what at first may appear to be a somewhat barbaric approach to theory development. However this approach is not novel, but is instead supported by Kelly's own elaboration of theory development. Kelly (1955) establishes clearly that he is skeptical about simply copying theories that have been designed for application with a different "foci of convenience" (p. 23). Instead his approach has been to discover the principles that are 
common to existing theory that can then be applied to the process of building new theories. ${ }^{9}$ Kelly's (1955) position can be seen clearly in this passage.

We are skeptical about the value of copying ready-made theories which were designed for another foci of convenience... Our position, then, would be that we should examine a variety of scientific theories, not to find one which can be copied concretely, but to discover common principles which can be applied to the building of brand-new theories. (p. 23)

In light of this, it is important to apprehend the similarities between the foci of convenience of Hermeneutic Constructivism and the Psychology of Personal Constructs, from which it borrows many of its principles.

As already elaborated, the focus with which Personal Construct Psychology concerns itself is the setting down of a theoretical position from which to apprehend the psychological domain of the business of being human. Somewhat parallel to this, Hermeneutic Constructivism outlines a theoretical position for discovering human experience at the level of the very structures that condition our internal experiences for the purpose of qualitative research. Undeniably both of these approaches are concerned with the psychological functioning of human being and any attempt to undermine these parallels would be foolhardy to say the least and hubris at worst. Instead the common focus upon the psychological functioning of the person means that the principles that are inherent within the Psychology of Personal Constructs provide fertile ground for extrapolation in the development of Hermeneutic Constructivism. Similarly, with regards to the fact that for Hermeneutic Constructivism it is language that conditions the psychological functioning of the person, parallels can be drawn with the principles of Gadamer's (1960/2003) Hermeneutic Phenomenology which locates language as the essential medium of being.

Although Hermeneutic Constructivism has extrapolated the principles that are inherent within existing and established theory, the success of a theoretical position is ultimately gauged in terms of its ability to have achieved the goals set for it. Despite this the success or otherwise of the Hermeneutic Constructivist position is difficult to assess in advance of its application. Usefully, however, Kelly (1955) outlines what he considers to be

\footnotetext{
${ }^{9}$ Support for Kelly's openness and indebtedness to those scholars that engage in the theoretical development of the Psychology of Personal Constructs can be seen in a recent article by Hinkle. Hinkle, a student of Kelly - most renowned for his work on Laddering - describes Kelly's genuine joy at seeing someone work with his theory and develop something new. Hinkle (2010) in a paper titled ' $A$ brief glance backward' wrote, "What interested me most was my theoretical extension of Kelly's work. Kelly said this had pleased him more than my methodologies" (p. vii).
} 
the "criteria of good scientific theory" (p. 24), which can be applied to the Hermeneutic Constructivist position independent of its application. Several of the criteria that Kelly (1955) describes have specific relevance to the focus of convenience of Hermeneutic Constructivism and therefore provide a framework to make a preliminary assessment of the qualities of this position. Each of the following criteria of good theory: Validity, Generality, Lawfulness and Individuality will now be discussed in relation to Hermeneutic Constructivism.

\section{Validity}

It is important that Hermeneutic Constructivism sets down a consideration of the way that information gathered about the subject matter is deemed an appropriate representation of the actuality of that subject matter. In other words, how can people using a Hermeneutic Constructivist position overcome any claim to providing little more than relativistic claims about the person of focus? Typically these issues involve consideration of the notion of validity, which embodies two fundamental considerations: (i) the degree to which the theoretical position can be considered to represent the subject matter of focus, and (ii) the degree to which this representation can be considered to meaningfully represent subject matter that is outside the confines of the study. Given that Hermeneutic Constructivism conceives of these two notions of validity in a somewhat different manner than traditional approaches, they stand in need of elaboration.

In terms of the first criterion, (i) the degree to which the theoretical position can be considered to represent the subject matter of focus, the theoretical propositions of Hermeneutic Constructivism are conceptually strong. Hermeneutic Constructivism has established that the subject matter of focus is the experience of the person. This is abstracted at the level of the structures that condition the qualities of his or her internal experience. This structure is the prejudice. Unpacked in terms of the most fundamental ontological structure of being human, the prejudice provides entrée to the dialogue of languaging and is the condition of our inner experiences or qualia. Thus, given that Hermeneutic Constructivism seeks to discover the structures that condition human experience or qualia, it is the uncovering of the prejudice that is of central concern.

Hermeneutic Constructivism has established that the comportment of being human is toward understanding. Thus it is here that the prejudice achieves its genuine ontological significance. It is the prejudice that provides the initial understanding from which the dialogue of languaging that sustains this comportment has its origins. It has also been established that the essential medium of this comportment is language. In other words, the structures that inform and sustain the understanding process are not different from the way 
that they come to language. The prejudice is no exception. Therefore, situating the essential structures of understanding as inextricable from language means that the person of Hermeneutic Constructivism is able to be articulate about his or her understanding processes and therefore the way he or she feels, his or her emotions and his or her experiences and perhaps most importantly - his or her prejudices. Thus, Hermeneutic Constructivism has indeed set down a theoretical position that is capable of discovering the subject matter of focus, the essential structures that condition a person's inner experiences, his or her prejudices.

With regard to the second criterion (ii) the degree to which this representation can be transposed as meaningful onto subject matter outside that seeking to be represented, Hermeneutic Constructivism is clear that the focus of consideration is the individual person. Underpinning this focus is a belief that each person differs with regard to his or her understanding processes. That is each person has an inherently idiosyncratic system of prejudices that not only provides entrée to the dialogue of languaging in particularly nuanced ways, but also provides access to his or her unique authentic consciousness that sustains and nourishes the ongoing dialogue of languaging itself. ${ }^{10}$ Ultimately it is the prejudice that conditions the possibility of human understanding and therefore the possibility of qualia. The inherently unique nature of the prejudices, and the connections that these prejudices make in a person's authentic consciousness, subsequently determine that person's understanding processes of everything that is able to be understood of the world, him or her self, and other people. Thus, on the basis of this understanding process, each person can be considered idiosyncratic.

While it is acknowledged that two people may language their prejudices in a similar way, this similarity would not persist beyond one or two prejudices and would most certainly not extend to a complete system of prejudices. It is for this reason that the focus of consideration here is unapologetically the individual person. As well as this, the prejudice as the most fundamental ontological structure represents all that a person already has and that can be brought to understanding of the world. In other words, a person's prejudices represent the Self. Therefore, at this level of abstraction, seeking comparisons of one person's

\footnotetext{
${ }^{10}$ The indissolubility of our understanding processes and the medium of language, as embodied by the theoretical elaboration of Hermeneutic Constructivism, credits the individual person with the capacity to be explicit about his or her prejudices, feelings, values, thoughts, and core beliefs. Here one is reminded of the incommensurate relationship between language and the construct identified within The Psychology of Personal Constructs and exemplified through the lens of Noaparast's (1995) and Stevens' (1998) positions of realism. The implications of the way in which Kelly inadequately dealt with language represents a theoretical hurdle that is well cleared by Hermeneutic Constructivism. A more considered discussion of the connection between Hermeneutic Constructivism and such realist positions is to be elaborated later this chapter.
} 
prejudices with another person's prejudices in order to establish validity is a philosophical faux pas.

This discussion could be misread as a disregard for the centrality of validity within Hermeneutic Constructivism. On the contrary, the area of validity considered most commensurate with the Hermeneutic Constructivist position is a consideration that is closely aligned with the first criterion outlined above, and is best posed as a statement. That is, good theory is explicit about the degree to which what a person says represents the actuality of his or her being. That is, it makes explicit how the dialogue that goes on in the enterprise of qualitative research is linked to the participant's prejudice. Here validity is not a consideration of the extent to which the prejudices of one person are confluent with those of another person. Instead, given that the person of Hermeneutic Constructivism is able to be articulate about thoughts, feelings, as well as core beliefs and therefore his or her prejudices, ${ }^{11}$ it is the dialogue of languaging that is of most central concern in terms of validity. In other words, it is within the dialogue of languaging itself that the prejudices of a person can be discovered. Despite the centrality of validity, Hermeneutic Constructivism is a theoretical position that has been created in an effort to apprehend differently an understanding of the inner outlook of a person going about the business of being human for qualitative research. If this position fails to serve this purpose, then the degree with which Hermeneutic Constructivism is deemed valid is meaningless.

\section{Lawfulness}

For Kelly (1955), good theory should be clear about the way in which those aspects of the world that fall within the domain of the theory relate to each other. Those aspects that fall within Hermeneutic Constructivism are the understanding processes of the person whose comportment is always towards understanding. However, prior to undertaking a consideration of the lawful nature of Hermeneutic Constructivism itself, it is useful to be clear about the type of theory being discussed. Here Hermeneutic Constructivism could be considered in terms of two theoretical positions: (i) a theory that provides a theoretical position whereby one person can come to understand another person, and (ii) a theory of what it is that propels the person.

\footnotetext{
${ }^{11}$ One must recall that the extent to which we can be explicit about our Authentic Consciousness is a function of the prejudice with which we gain access to it. It is inconceivable for Hermeneutic Constructivism that a person can elaborate everything that he or she known about every possible thing there is to know something about, without the prompting of questions. For example, the question 'what do you know about...?' provides a conduit sufficient to provide a pathway into authentic consciousness and that which is understood about that thing thus far.
} 
It must be said that we can indeed bring to understanding aspects of a complex and lawful system without having to appreciate the laws that propel it. Take for example a tree. A tree grows and develops according to the mathematical laws set down by Leonardo of Pisa, known more commonly as Fibonacci. Unquestionably it is possible for us to simply gaze at the majesty of the tree without giving a second thought for the laws that put it there. However, if we were to attempt to genuinely understand the inner workings of the tree, to understand it more deeply, then we may indeed be driven by a need to understand these laws. So too the subject matter of concern here, the person. Certainly we can stand back from and simply admire a person, yet to genuinely begin to understand that person we must appreciate the principles that constitute and represent that person. Given that the comportment of human being is always toward understanding, and that it is the prejudice that ultimately conditions understanding, then it is a person's greater system of prejudices that represents the essence of that person's being. Thus the greater prejudice system represents an internal theory of a person, and it stands in need of consideration here in relation to the way in which these structures operate lawfully.

As previously discussed, the Organisation Corollary embodies the notion of lawfulness within a person's greater system of prejudices. It takes as its starting point the view that each prejudice is an integral component of a greater system of prejudices. The nature of this relationship is such that the adjustment of a prejudice that is found to be misleading is always made through the dialogue of languaging itself and guided by the implicit lawfulness of the greater system of prejudices. That is, the superior prejudices within a person's hierarchical system of prejudices provide that person's system of prejudices, and therefore his or her understanding processes, an implicit degree of consistency. It is for this reason that the Self carries a similar degree of consistency. In the absence of this consistency the world would appear as a swirling mass of light and sound that would be indiscernible. Thus the movement from one prejudice to another is sufficiently stable so as to be more consistent than happenstance alone. In fact it is the inextricable links between the prejudice and authentic consciousness that provide the foundation for this consistency.

It has been established that authentic consciousness encompasses everything that we already have about a thing to be understood. It follows, therefore, that authentic consciousness also represents the touchstone for all that we do not know. In other words, recognition of what we do know is in itself also recognition of what remains unsettled about a thing to be understood. Recognising those aspects that remain unsettled in our understanding provides us with an avenue to pose further questions, and in so doing develop our understanding. It follows that the questions asked of the thing are therefore what determines 
the direction of a dialogue and indeed the prejudices that are evanesced towards. More specifically, the questions that are asked are in fact questions of those aspects of the authentic consciousness that a prejudice opens up toward. A return to the first love example is again useful for unpacking this idea further.

In the first love example, the person projected the prejudice, overwhelmingly positive emotion, in trying to understand a thing that is clearly happening but is not as yet understood. Until this time in the person's life he or she has not had this emotion. Thus those aspects of authentic consciousness that the prejudice overwhelmingly positive emotion open up toward are not able to embody adequately all that is happening. Through the dialogue of languaging this prejudice gradually evanesces towards another prejudice. Recall that when the limits of what a person is able to envision from the horizon of a prejudice is reached, he or she evanesces to another prejudice, and the comportment of being continues. Thus, here in the dialogue of languaging, this person may come to an understanding of this initial prejudice overwhelmingly positive emotion - in terms of 'it is different from anything I have experienced before'. When the limits of the horizon of that prejudice are reached, this person may adopt the vantage point of a different prejudice, perhaps a prejudice, I cannot stop thinking about this person. In turn, this prejudice will provide access to different aspects of authentic consciousness and the subsequent possibilities of the ongoing dialogue of languaging.

\section{Generality}

An understanding of what Kelly (1955) meant by the notion of 'generality' is perhaps best captured by the posing of a question: Can the highest order structures that a theoretical position concerns itself with be traced through nearly all phenomena that a theoretical position has established itself to deal with? In terms of Hermeneutic Constructivism, then, can the prejudice be traced through this theoretical unpacking of human experience and understanding?

Hermeneutic Constructivism has established that the comportment of being is toward understanding. Here we project a prejudice toward the world, illuminating the world and subsequently determining the adequacy of that light in order for us to go on within the world. Thus it follows that in the theoretical unpacking of Hermeneutic Constructivism the prejudice represents the fundamental ontological structure from which all understanding originates. The prejudice is the essential condition of understanding itself. In fact, it is the prejudice that conditions our internal experience and provides the possibility of having qualia. Thus the prejudice is the highest order abstraction through which the person envisaged by Hermeneutic 
Constructivism can be considered. The inextricability of the prejudice from language means that the prejudice itself represents a structure that can indeed be traced through the phenomena of interest, an understanding of human experience.

\section{Individuality}

Fundamental to the development of a sound theoretical position for Kelly (1955) is the ability of that approach to explain individual differences in a lawful manner. He argues that the outlook of the individual person is in itself a real phenomenon and therefore must be viewed as such. Thus each person should be considered in terms of his or her view and not from the perspective that what one person thinks has to be like another or even like the greater public opinion. As a function of this, Kelly (1955) recoils at the idea of using group data to infer the status of the individual person, a view made clear in this passage.

We cannot of course crawl into another person's skin and peer out at the world through his eyes. We can, however, start by making inferences based primarily upon what we see him doing, rather than upon what we have seen other people doing. (p. 42)

In emphasising individuality, Kelly (1955) proposes a careful consideration of the relationship between private and public domains. Here he suggests that theory should concern itself with the abstraction of data of a particularly high order. For him the highest order is at the level of the individual person. As a function of this, he argues that the primary focus of consideration should be the individual person, prior to reducing that person to simply a datum in the study of a group of people. ${ }^{12}$ In order to emphasise how it is that the person of Hermeneutic Constructivism can be considered to function lawfully in both private and public domains, a brief re-sketch of how this person experiences and understands the world is useful.

There can be little argument that each person has experienced a great many things, in and of the world, that are inherently unique to him or her. In other words, each of us has directed our understanding processes toward an amazing array of idiosyncratic events of understanding. Thus we have an inherent self-determinism in our being with the world. Not only are the events of understanding that we choose to direct our understanding processes towards decidedly idiosyncratic, but also that something that we make of those events is

\footnotetext{
${ }^{12}$ Kelly (1955) sums up the argument concerning the consideration of both the private and public domains of human existence as follows; "If a man's private domain, within which his behaviour aligns itself within its own lawful system, is ignored, it becomes necessary to explain him as an inert object wafted about in a public domain by external forces, or as a solitary datum sitting on its own continuum. If a man's existence in the public domain is ignored, our painstakingly acquired knowledge of one man will not help us understand his younger brother" (p. 39).
} 
fundamentally unique. At the core of this view is an acknowledgment that what each person makes of the events of time-past is ultimately determinant of the possibilities that the person has available for envisaging the world in time-present. A closer examination of what goes on in the processes of making something of an event, will underscore the idiosyncratic nature of being for Hermeneutic Constructivism.

These idiosyncratic somethings that are made of the events of understanding determine a person's authentic consciousness and ultimately his or her prejudices. It is the prejudice that not only provides entree to the dialogue of languaging, but also to authentic consciousness. In fact this dialogue of languaging that the prejudice provides entree to, is the same dialogue that operates to shape the prejudice, and subsequently those aspects of authentic consciousness that are ultimately opened up toward in the future. These inextricable links between the prejudice, authentic consciousness and the human comportment toward understanding, emphasise the fundamentally idiosyncratic nature of being. In other words, as a function of the illuminative qualities of the prejudice and subsequent aspects of authentic consciousness, the person of Hermeneutic Constructivism is considered to develop a view of the world that is indeed his or her own.

Having established how the person of Hermeneutic Constructivism can be considered unique or individual, it must be established how this same person can be considered in the public domain. That is, being theoretically reflexive to the way in which each person does not go about the business of being human in silence. Instead each of us always has the capacity of sharing our innermost feelings, attitudes, beliefs, experiences and emotions with other people. Having already established that the prejudice and subsequently those aspects of authentic consciousness or the horizon of the prejudice(s) are inextricable from language, it follows that the person envisaged here is certainly able to be articulate about his or her idiosyncrasies. In order to bring a thing to understanding, a person searches for the Mot Juste as the very condition of the qualities of our inner experiences. In so doing, this person can carry forward the meaning of his or her understanding through the same Mot Juste for another person. It is this inextricability of the qualities of our inner experience from language that allows the individual to be considered in the pubic domain.

It has already been outlined that the prejudice, as the highest order abstraction with which the person of Hermeneutic Constructivism can be considered, operates in a consistent and lawful manner. It is this lawfulness of a person's prejudice system that embodies the notion of Self, and therefore the inherent individuality that is envisaged of the person. Thus the inextricability of these idiosyncratic structures of understanding from language provides a theoretical approach that meets Kelly's (1955) requirements for a “ constructive approach to 
the relationship between private and public domains" (p. 39). Despite this emphasis upon the nexus between the private and the public domains, it is important to appreciate that the primary focus of Hermeneutic Constructivism is the uncovering and elaboration of a person's prejudices. It is not the primary concern of Hermeneutic Constructivism to set down a series of guesses as to the way the prejudices from one person bear similarity with those of another person.

\section{Elements of Theory}

The discussion that follows from this title is perhaps incongruent with the location in which the reader finds him or her self. However, the concern here is not with theory itself. Instead, it is with what can be expected of Hermeneutic Constructivism now that it has been posited. A useful starting point is the consideration of what type of theory Hermeneutic Constructivism actually is. The notion of a theory is most synonymous with the binding together of a collection of facts in order to make sense of them. Therefore, if envisaged from this perspective, where the subject matter of interest is the understanding processes of the individual person, then that person would necessarily be conceived as having the capacity for going about the world and simply collecting, and binding together, these facts. However such an approach lends itself to a passive view of human experience. Given that the person envisaged by Hermeneutic Constructivism is always proactively engaged in the world, a more active conceptualisation of theory is required in order to more adequately capture this activity. Kelly (1955) provides a clear description of what he believes an active theory to be, and goes on to emphasises the importance of a theoretical position to apprehend this activity.

A theory provides a basis for an active approach to life, not merely a comfortable armchair from which to contemplate its vicissitudes with detached complaisance....Men can play active roles in the shaping of events. How they can be free to do this and still themselves be construed as lawful beings is a basic issue in any psychological theory. (pp. 18-19)

Despite the focus of this description on the psychological functioning of a person, it has been established previously that Hermeneutic Constructivism is not diametrically opposed to this focus. Instead there are some clear intersections between the way that Hermeneutic Constructivism unpacks the vicissitudes of human experience and the take on the psychological functioning of the person as captured by Kelly. Importantly Hermeneutic Constructivism is not a psychological theory per se, as a platform from which to make judgments about the satisfaction or otherwise of another person's psychological functioning. Instead it is a theoretical position that unpacks a possible account of the psychological or 
understanding processes of the person. Thus the range of this theoretical position, at this juncture, is not expected to exceed the informing of a deeper consideration of the individual person for the purposes of qualitative research. Moreover, although Hermeneutic Constructivism begins from the philosophical acceptance that the person envisaged comes to understand the world through the medium of language, as set out in the unpacking of Person the Languager, much like the Psychology of Personal Constructs it does not represent a fully fleshed out philosophy.

Having established what Hermeneutic Constructivism is not, a consideration of what indeed $i t$ is stands in need of elaboration. Hermeneutic Constructivism represents a theoretical position from which to contemplate the person going about the business of being human for the purpose of qualitative research. A contemplation that takes as its focus a particularly high level of abstraction, the prejudice. To paraphrase Kelly, what is of tremendous interest to the Hermeneutic Constructivist position is to contemplate the way in which a person plays an active role in the shaping of his or her experience and understanding of the world. Of equal importance is a consideration of how this same person can be considered to operate lawfully as a function of the structures that condition the process of understanding itself. Also important is the way that these structures are able to carry meaning to other equally active people in understanding.

With this consideration of what the Hermeneutic Constructivist position represents in mind, attention can now be drawn to a consideration of what this theoretical position can provide. The preceding two chapters have amplified Hermeneutic Constructivism as a theoretical position from which to contemplate Person the Languager at a particularly high level of abstraction, the prejudice as the condition of our internal qualities of experience. Despite the aspirations held for the Hermeneutic Constructivist position, it must be conceded that some comfort is taken from the words of Kelly (1955) who suggests, "theory need not be highly scientific in order to be useful" (p. 18). Instead a theory should be developed with a focus upon the subject matter, and the representation of this subject matter should therefore be taken as the measure of the theory's overall success. Thus where that subject matter is the internal qualities of a person's experiences, then a representation to abstract datum at the level of the structures that condition this subject matter is a representation indeed.

Remaining consistent with the Hermeneutic Constructivist position herein, one is reminded that no thesis is devoid of an antithesis as the point at which a theoretical position is 
no longer useful. ${ }^{13}$ In fact a good theory, or rather a good theorist, acknowledges that any theoretical position is expendable if confronted with contrary evidence. More than this, however, good theory should open up opportunities for exploring phenomena differently, as well as providing the impetus for dialogue. Thus Hermeneutic Constructivism is not proposed as 'The Theory'. Instead it represents a possible solution to a series of identified problems. Therefore, if this solution provides entrée to further dialogue, then Hermeneutic Constructivism is a theory indeed. In line with this, Kelly (1955) was most pragmatic about theory development, and emphasised the potentially finite nature of a theoretical position. ${ }^{14}$

Any theory, then, tends to be transient. And the more practical it is and the more useful it appears to be, the more vulnerable it is to new evidence...If the theory we construct works well within this limited range of convenience, we shall consider our efforts successful, and we shall not be too much disturbed if it proves to be less useful elsewhere (pp. 14, 23)

Although the founded capacity of Hermeneutic Constructivism to open up further opportunities for dialogue would be a most gratifying outcome, it is a measure that cannot be known free from its application. In light of this, a turn is now made towards the way that we would bring to fruition qualitative research outcomes that are informed by the theoretical tenets of Hermeneutic Constructivism. Prior to this, however, it is useful to attend to the problems that were identified earlier within The Psychology of Personal Constructs, and in so doing highlight the possibilities that Hermeneutic Constructivism provides in overcoming these problems. After all, Hermeneutic Constructivism has been described previously as a theoretical position that has as its origin in a re-envisioning of the Psychology of Personal Constructs from the perspective of Gadamer's hermeneutic phenomenology.

\section{A Hermeneutic Constructivist Lens on Personal Construct Psychology}

It has been established previously that the compatibility of the Psychology of Personal Constructs with a realist position is difficult to maintain given the way in which Kelly dealt poorly with language. This identification of the Psychology of Personal

\footnotetext{
${ }^{13}$ The notion of gravity is a particularly good example. Newton was perplexed by the force that makes an apple fall from the tree, and suggested that it was indeed the same force that kept the planets in orbit. However, Einstein's theory of General Relativity, the growth of 'quantum mechanics' and more recently String Theory all extend beyond Newton's initial and fundamental understanding. The point being, even great theory has the possibility of one day being superseded in the continuum of time.

${ }^{14}$ Kelly (1955) believes that it is as important to have a practicable theory, as it is to have a theoretical position that is open to change. He suggests that good theory "should also encourage the invention of new approaches to the solution of the problem of man and his society" (p. 24). It is with the production of such new approaches to understanding human being and society that Hermeneutic Constructivism concerns itself.
} 
Constructs with realism is important for its adherents to establish in order to be assured that it is indeed a real world that a person discloses by way of his or her constructs. However the tenuous nature of the links between the construct, world and language raises questions about the theoretical tenability of Kelly's (1955) approach, and more specifically the sustainable nature of the construct itself. These questions ultimately challenge the unabridged adoption of Kelly's (1955) approach as a theoretical position for informing qualitative research. Given that the core deficit that has been identified in Kelly's (1955) works was language, it is in the direction of language that an elaboration of Kelly's work was pursued. The hermeneutic work of Gadamer (1960/2003), and his account of the inextricability of language from being, provided a theoretical platform for re-envisioning Personal Construct Psychology. The product of this re-envisioning is Hermeneutic Constructivism.

Developed as a response to the challenges identified within Kelly's (1955) approach, it is thereby important to consider the extent to which Hermeneutic Constructivism can be seen to overcome these challenges. This entails considering the way in which Hermeneutic Constructivism discloses a real world through the structure of the prejudice and its subsequent elaboration from authentic consciousness. In light of this, a consideration of the degree to which Hermeneutic Constructivism is compatible with the same criteria of realism against which the Psychology of Personal Constructs was considered, and subsequently found to be incompatible, will be provided. Therefore, in order to determine the extent to which Hermeneutic Constructivism can claim to have overcome the issues of incompatibility identified between Kelly's (1955) position and Noaparast's (1995) Sophisticated Realism, a brief sketch of this incompatibility is useful. ${ }^{15}$

As outlined previously, Noaparast (1995) sought to overcome the challenge that the Psychology of Personal Constructs is little more that an instrumentalism that succumbs to unsustainable simple realism. ${ }^{16}$ At the core of this challenge is a view that ideas, and more

\footnotetext{
${ }^{15}$ For a more complete sketch of the theoretical hurdle identified within The Psychology of Personal Constructs, refer back to Chapter Four.

${ }^{16}$ It could be argued that Noaparast's (1995) position is but one of many realist takes that are possible within the Personal Construct Psychology literature (Stojnov \& Butt, 2002). As mentioned previously, Stevens (1998), also commonly cited in this area, is openly indebted to Noaparast's (1995) work and can be considered to bolster his position further. Moreover Warren (1998), a key figure within the philosophical considerations of Personal Construct Psychology, acknowledges Anderson's (1960) work as "the most developed realist position" (p. 52), and emphasises that the similarities between this position and Noaparast's (1995) sophisticated realism are noteworthy. Although Warren (1998) goes on to suggest that Anderson's (1960) approach is by some readings less commensurate with Personal Construct Psychology, he believes Noaparast (1995) provides a suitable touchstone for the way in which the Psychology of Personal Constructs can be considered consistent with a realism. The point to be made here is that in pursuing the work of Noaparast (1995) as a realist backdrop, we can be assured of confronting the most central arguments that inform a realist conceptualisation of Personal Construct Psychology. As well as a realist touchstone, Noaparast's (1995) sophisticated realism provides a useful
} 
specifically constructs, are merely tools that a person uses to work on the world. However these tools ultimately require the theorising of a simple realism or a correspondence between the construct and the world. In response to these challenges, Noaparast (1995) proposes Sophisticated Realism. ${ }^{17}$ Based heavily upon the neo-pragmatism of Quine (1960), Noaparast (1995) elaborates five core tenets upon which his sophisticated realism is posited. These tenets are: (i) there is a real world independent of our theorising; (ii) our theories can account for this reality; (iii) fact-theory distinctions are not acceptable; (iv) any simple correspondence between theories and reality is rejected; (v) our true sentences correspond to the reality of the world captured by our best theories. These tenets provide a useful backdrop against which to now consider the advances made by the Hermeneutic Constructivist position. ${ }^{18}$ Here each of the five tenets will be re-considered in terms of Hermeneutic Constructivism in order to emphasise the advances made by this theoretical re-envisioning of Kelly's (1955) position.

\section{(i) There is a real world independent of our theorising}

It is important to acknowledge from the outset that any position taken with regard to this proposition remains entirely speculative. That is, if it were possible to establish beyond doubt that a world existed independently, without the need for any degree of theorising, the extreme realists would triumph in this longstanding philosophical debate. It is, however, not possible to establish the existence of a real world independently of a theoretical disclosure of it. In light of this, Hermeneutic Constructivism sets down a theoretical view contending that it is a real world in which we live, and that our languaging efforts operate to allow that world to come to understanding. In sustaining this argument, the burden of proof is insurmountable. So much so that we are compelled to defer to the use of an obviously argument in order to

vehicle for highlighting a need for the theoretical development of Personal Construct Psychology with regard to the connections between language, construct and world - a position ultimately achieved by Hermeneutic Constructivism.

${ }^{17}$ It must be acknowledged that Stevens (1998) also developed a realism called a Minimum Realism. However, given the open indebtedness of Stevens (1998) to Noaparast's (1995) work, it is Sophisticated Realism that will be used for exploring the way that Hermeneutic Constructivism can be considered to redress the challenges that have been identified previously in the incompatibility of Kelly's (1955) work with Noaparast's (1995) position.

${ }^{18}$ Noaparast's (1995) sympathetic undertones towards a phenomenal conception of the world provide further support for its use as a backdrop for considering the merits of Hermeneutic Constructivism. However, given that Hermeneutic Constructivism has established understanding in terms of the ontological comportment of being human, and the subsequent indissolubility of the person and the thing that he or she reaches towards in understanding, the engagement in an argument concerning the compatibility of Hermeneutic Constructivism with a realist position is perhaps a somewhat redundant affair. Nonetheless, against the backdrop of Noaparast's (1995) tenets of Sophisticated Realism, one can clearly identify the theoretical advances made by Hermeneutic Constructivism in addressing the challenges levelled at The Psychology of Personal Constructs as a theoretical position for underpinning qualitative research. 
progress the discussion. Thus, to paraphrase Warren (1998), obviously "we do not just dialogue, we dialogue about something" (p. 58), we dialogue about the world.

\section{(ii) Our theories can account for this reality}

Having laid claim to the existence of a real world that is independent of our theorising, the aspect of this tenet that remains in need of consideration is the phrase 'our theories'. In relation to Hermeneutic Constructivism, a consideration of the prejudice is again useful. It is the prejudice that provides the essential ontological prejudgment that illuminates the thing to be understood, and thereby allows it to come to understanding. So much is this the case that the dialogue of languaging that ensues from this initial position represents a person's take on the thing that is to be understood. In following this argument, a person's greater system of prejudices represents the Self and therefore the possibilities of his or her take on the world.

In order to apprehend how a theory is synonymous with a person's greater system of prejudices, a return to Stevens' (1998) commentary on Noaparast's (1995) work is needed. Stevens (1998) suggested that there is more than simply an analogy between Noaparast's (1995) notion of a theory and Kelly's (1955) notion of a construct. In fact Stevens (1998) goes on to state, "taken as a whole, the construct system operates much like a personal theory of the world" (p. 295). Thus, in following Stevens (1998), but with regard to Hermeneutic Constructivism, our greater system of prejudices represents our best theory of the world. Therefore, given the essential ontological nature of the prejudice, our system of prejudices, as our theory of the world, more than adequately accounts for the reality of the world. In fact the prejudice of Hermeneutic Constructivism more than accounts for the world, it is the essential precondition of having a world at all.

In building upon this idea it must be stated that this notion of 'accounting for this reality', as outlined by this tenet, is less commensurate with the Hermeneutic Constructivist approach than with the Psychology of Personal Constructs. Providing an 'account of something' implies that the account is in some way different from the thing that is being accounted for. This would mean that the account is therefore something different from the world, thereby entailing a subject/object dualism. Indeed it has been suggested that the way in which Kelly dealt poorly with language creates a clear delineation between the world and a person's construction of it. That is, the person of Personal Construct Psychology must discover the constructs that account for or conform to the world by use of a mechanism that is outside of language. In contrast to this, for Hermeneutic Constructivism the structures of our understanding processes are not different from language. Therefore, the way in which a thing 
of the world comes to language is not different from the thing itself for us. This proposition overcomes the separation between an account of the world, and the world, that is implicit within the Psychology of Personal Constructs. Instead Hermeneutic Constructivism ultimately reconnects the subject with the object. For Hermeneutic Constructivism the notion that our theories can account for reality is better captured by the idea that our theory is reality itself for us.

\section{(iii) Fact-theory distinctions are not acceptable}

The way in which Kelly dealt poorly with language, situating the construct beyond the reach of language, establishes a clear distinction between the fact of the world and a person's theorising, or theory itself. ${ }^{19}$ The following passage from Kelly (1955) highlights the way that a person's construct system can be likened to a series of 'scanning patterns'.

When a person scans the events with which he is surrounded he 'lights up' certain dichotomous construct systems. Thus construct systems can be considered as a kind of scanning pattern which a person continually projects upon his world. As he sweeps back and forth across his perceptual field he picks up blips of meaning. The more adequate his scanning patterns the more meaningful his world becomes. (p. 145)

Here Kelly is clear that a person's constructs are something different or distinct from the world. Kelly thus failed to elaborate a medium of being human, or a medium within and through which the world comes to understanding and yet is at the same time the medium of the construct itself. In so doing, his construct system must exist independently of the world as something that is other, as something that is simply tried on the world in order to determine what aspects of the world can come to 'blips' of meaning. Thus there is a clear distinction between a person's theory or constructs and the world.

In contrast, Hermeneutic Constructivism situates language as the inextricable medium of the human comportment toward understanding, of being human. It is language that is therefore the medium through which a person has a world at all. In other words, for Hermeneutic Constructivism, a thing that cannot come to language cannot be understood. Thus the facts of the world are not different from the way in which they are brought to language in the dialogue of languaging itself. This represents a position that operates to overcome any simple fact-theory distinction.

\footnotetext{
${ }^{19}$ For a more complete discussion see Chapter Four.
} 
It has also been established that the prejudice is an initial prejudgment that illuminates the world, and from which the dialogue of languaging begins. Thus the prejudice represents the possibilities of what can become meaningful in the dialogue of languaging. This view is in contrast to simply achieving an understanding of what is supposedly already meaningful. That is, the prejudice that is projected towards the world does not seek a correspondence with the thing to be understood in the same way that a construct does. Instead the prejudice always provides entrée to the dialogue of languaging and opens up ongoing 'is it...?' type questioning. This type of questioning is always directed towards more adequately accenting aspects of the thing in order to go on being. On the other hand, the construct opens up 'where is it...?' type questioning. Here the person envisaged seeks, or rather scans, for a correspondence between world or fact and a construct or theory. Thus the prejudice is not an instrument in the way that the construct is. Instead, the prejudice is the initial position from which the comportment of being always begins. Therefore, the way that a thing is understood is in fact not different from the way in which it is brought to language through our system of prejudices or as our best theory.

\section{(iv) Any simple correspondence between theories and reality is rejected}

A most suitable beginning point here is a recapitulation of what is meant by the term correspondence. ${ }^{20}$ A correspondence is an accord, or a concern for the degree to which something is representative of something else. More specifically, it is a concern with the degree to which a theory accords with reality. The correspondence that Kelly (1977) envisaged between a person's construct system and reality is made patently clear in the following passage.

What we think we know is anchored only in our own assumptions, not in the bed rock of truth itself, and that world we seek to understand remains always on the horizons of our thoughts. To grasp this principle fully is to concede that everything we believe to exist appears to us the way it does because of our present constructions of it. (p. 6.)

As has already been established, and is again palpable here, without the capacity of the construct to come to language, and to engage in a dialogue with the world, Kelly's position can be little more than a simple correspondence.

\footnotetext{
${ }^{20}$ The notion of correspondence poses a philosophical conundrum. To be in a position to make a judgement about the accord of a theory with reality assumes that one can know reality first and foremost. As mentioned in the elaboration of the first of Noaparast's (1995) five tenets earlier this chapter, a belief in a correspondence theory is but one of a host of possible 'takes' on the actuality of human understanding.
} 
In terms of Hermeneutic Constructivism, the concern is with the degree to which our theory or system of prejudices can claim an accord with the reality of the world itself. The way that Hermeneutic Constructivism has situated language as the medium of being human means that the world always has the potentiality of becoming to understanding. The use of this word 'becoming' here captures the point that although the world lends itself to being understood, for Hermeneutic Constructivism our understanding of the world can never be complete. Instead, understanding is always in the process of becoming a different understanding. ${ }^{21}$ Thus, in the dialogue of languaging, the thing to be understood becomes a thing of itself for us, in a manner that is not different from the way in which it comes to language. In other words, what we bring to understanding in languaging cannot be considered in terms of a correspondence or accord with something else. Rather, it is the world, as all that it can be at that moment for us, it is the world for us thus far.

\section{(v) Our true sentences correspond to the reality of the world captured by our best theories.}

Given that much has already been said about this tenet in relation to the Psychology of Personal Constructs previously, the focus at this point is to provide a consideration of the compatibility of this final tenet with Hermeneutic Constructivism. ${ }^{22}$ In order to facilitate this consideration, several key elements that are consistent with Hermeneutic Constructivism will be adopted as a framework for unpacking this compatibility further. These elements are as follows: (a) Language as Medium not as Instrument of Correspondence; (b) Historicity of the Prejudice as One's Best Theory; and (c) The Carriage of One's Theory.

\section{(a) Language as Medium not as Instrument of Correspondence}

Despite Noaparast's (1995) adherence to the capacity of a person to bring his or her best theory or construct system to language, this view has been shown to be in contradiction with Kelly's (1955) work. In fact, Kelly (1955) is unambiguous that the person he envisages does not have the capacity to bring to language a single construct let alone his or her greater system of constructs. Thus a close reading of Kelly's (1955) work suggests that The Psychology of Personal Constructs is in fact incompatible with a Sophisticated Realism,

\footnotetext{
${ }^{21}$ Each day we can witness the evidence of this in the way captured by the Passive Corollary. The world happens at each moment of the day and although we could actively make something of the world, we are simply unable to do so. This does not mean that the world stops when one stops making something of it, rather it simply indicates that the world has a potential of being understood if one actively chooses to.

${ }_{22}$ Please refer to Chapter Four for a more comprehensive review of the challenges this tenet entails for Personal Construct Psychology.
} 
given that a person cannot bring to language his or her best theories. ${ }^{23}$ Being situated beyond the reach of language, any position that suggests - as Noaparast (1995) does - that a construct can indeed come to language, would be unable to avoid a subscription to a simple realism, or correspondence. However, to assume the possibility of such a correspondence is clearly incommensurate with tenet $i v$ of Noaparast's (1995) Sophisticated Realism and more specifically with Kelly's (1955) theory itself.

The Fundamental Postulate of Hermeneutic Constructivism, on the other hand, takes as its starting point the notion that language is the medium of the business of being human. In so doing, it emphasises that language is not simply a 'tool' or 'instrument' that we use to work on the world. Instead, language is disclosing of the world, and is the way that we have a world at all. In adopting such a position Hermeneutic Constructivism does not slip into an unsustainable correspondence or simple realist conception of truth. Instead what a thing is is not different from the way in which it comes to language. This theoretical maneuver goes a long way toward overcoming any need for a correspondence and situates Hermeneutic Constructivism firmly within the realist end of the realism-idealism spectrum.

This idea that the person of Hermeneutic Constructivism can simply bring his or her best theories to language may at first appear to be a slippage toward the idealism end of the spectrum. What is required of Hermeneutic Constructivism, then, in order to overcome such a charge, is to uncover the historicity that is implicit within our prejudice system or best theory. In other words, to highlight how time-past can be seen in time-present, and in so doing underscore how our best theory has an inherent consistency and persistence.

\section{(b) Historicity of the Prejudice as One's Best Theory}

Experience for Hermeneutic Constructivism is the dialogical movement of making something of the events toward which we direct our understanding processes, first and foremost our prejudices. Although the prejudice has been situated as determinate of what can come to be understood of an event, it is in fact the inextricability of the prejudice and authentic consciousness that is ultimately determinate of the potentialities of understanding. Authentic consciousness represents everything that we already have of a thing to be understood. Recall that each prejudice has a horizon that represents aspects of authentic consciousness that the prejudice provides access to in the ongoing dialogue of languaging. Conceptualised in this way, the structures of understanding carry an inherent historicity. That

\footnotetext{
${ }^{23}$ In fact the Psychology of Personal Constructs suggests that 'language is merely a tool' (1955, pp. $138,354 \& 1962$, pp. 4, 11, 14). A tool beyond the reach of which the construct has its being. In other words, for Kelly (1955) the construct exists outside of language.
} 
is, the way that a person illuminates the world in understanding has as its source the prejudice that exists prior - or anticipatory - to the event of understanding itself.

A final return to the example of a first love is here explanatory of this notion of historicity. In the example, the person projected the prejudice overwhelmingly positive emotion toward the sensations that were clearly happening but could not meaningfully be understood. Although we know, by having worked through this example previously, that this person culminates his or her understanding of these sensations in terms of this must be love, the latter is a position that only develops as a function of the dialogue of languaging. It was this initial prejudice, overwhelmingly positive emotion, that ultimately provided entrée to the dialogue of languaging and represents the Mot Juste or the most perfect phrase for capturing what it is that the person already had. That is, not having made something of an event of understanding previously in terms of love, the most adequate way that this person is able to bring that sensation to understanding is from the vantage point of the prejudice or prejudgement that he or she already has. Given that the dialogue of languaging has developed this prejudice to this must be love, future anticipations or projections of understanding may adopt the vantage point of this prejudice and all that it opens up toward in authentic consciousness. The point to be made here is that all understanding begins from an initial understanding, an understanding that is always mediated by the historicity of experience. That is, we always comes to understand a thing to be understood by way of that something which we have made of an event in time-past, as experience per se. ${ }^{24}$

\section{(c) The Carriage of One's Theory}

The preceding discussion has focused upon the way in which the bringing of a thing to language is not different from the thing itself and that the dialogue of languaging is sustained by the inherent historicity of our best theory. Despite this, the issue remains to apprehend the degree to which our true sentences correspond to the reality of the world captured by our best theories. In other words, is it possible for the person envisaged by Hermeneutic Constructivism to bring to language his or her prejudice system?

\footnotetext{
${ }^{24}$ It is worthy of a final note here that Kelly (1955) was adamant that Personal Construct Psychology was not to be conceptualised in terms of its historicity despite the anticipatory nature of his theoretical position. The following is illustrative of his view: "the perceptual theories in psychology are frequently said to espouse the ahistorical approach to the understanding of behaviour. This approach is from the viewpoint that one's activity at a given moment in determined primarily by his outlook at that moment. What has actually happened in the past can influence behaviour only through the perceptions which are operating at the present instant. Personal-construct theory takes a somewhat similar stand" (pp. 174-175).
} 
It has been established that for Hermeneutic Constructivism the way in which a thing is understood is not different from the way in which that thing comes to language in the dialogue of languaging. This conceptualisation is not different when that thing to be understood is our understanding processes. The processes of understanding includes those structures that are essential for making something of an event. These process of understanding include an essential coalescence of the prejudice, those aspects of authentic consciousness that the prejudice provides entrée to and the subsequent dialogue of languaging itself. Most centrally, given that each of these structures is inextricable from language, it follows that the person envisaged by Hermeneutic Constructivism is able to be articulate about them. That is, the way that these structures come to language is not different from the structures themselves. Thus, from the perspective provided by Hermeneutic Constructivism, it is indeed possible for our true sentences to represent our best theory or prejudice system.

The acknowledgement that a person can bring to language his or her system of prejudices is not to say that this same person could merely find a quiet spot and elaborate every detail of the way that they envisage the world. Nevertheless, Hermeneutic Constructivism takes the view that when prompted by a question, in a specific direction, a person can answer that question and in so doing provide detail of his or her prejudice system and those subsequent aspects of authentic consciousness accessed. Here one is asked to recall the exercise outlined previously, where you were asked to provide a delayed answer to the question, How are you feeling? In this exercise you may recall that until a response was provided to the question, the feeling itself did not become anything. ${ }^{25}$ Thus the way in which feelings or the qualities of your internal experience are brought to language, is not different from the way that these feelings are for you the reader. This is not a correspondence, it is a thing of itself, the way that it is for you. Therefore the point to be made here is that for Hermeneutic Constructivism it is possible for a person to represent his or her best theory of the world, not in terms of a correspondence but instead in the way that it is itself for that person - as a view of the world.

\section{Chapter Summary}

A re-envisioning of Kelly's Personal Construct Psychology through the lens of Gadamer's Hermeneutic Phenomenology yielded the Hermeneutic Constructivist position. This chapter has provided a descriptive elaboration of the previously outlined Fundamental

\footnotetext{
${ }^{25}$ This exercise is also reminiscent of Wittgenstein's (1968) 'Paper Trick', where he demonstrated that irrespective of how one tried, ultimately all meaning only occurs in language. For a consideration of this exercise review Chapter Six and the elaboration of the Fundamental Postulate of Hermeneutic Constructivism.
} 
Postulate and its amplifying corollaries. A consideration of the nature of the prejudice and the nature of languaging situated the prejudice itself, and the dialogue of languaging that it opens up toward, as the fundamental ontological structures of being human. In fact, the prejudice was described as the condition of the qualities of our internal experience, or our qualia. As well as this, the possibilities of how it is that we come to understand the world, our self, and other people, is fundamentally a function of the adequacy with which a prejudice as a Mot Juste - illuminates the thing that is to be understood. Thus the essential historicity that is inherent within the prejudice(s), supports the view that all understanding is interpretation, and in so doing acknowledges that our understanding is always and only an understanding as it is for us, an understanding thus far.

To suggest that the theoretical unpacking of the Hermeneutic Constructivist position appears to be conceptually sturdy does not overlook the importance of waiting until its application before making any final judgements. This suggestion comes from having undertaken a consideration of several of the key principles of good theory, as set down by Kelly (1955), against which Hermeneutic Constructivism appears to be sound. In order to emphasise the theoretical advances made by the Hermeneutic Constructivist position in overcoming the challenges levelled at the Psychology of Personal Constructs, Noaparast's (1955) Sophisticated Realism was used as a touchstone. Hermeneutic Constructivism has established that language is the medium of being human, to the extent that the historically mediated structures that condition a person's understanding processes are also inextricable from language. This inextricability can be seen to underscore an affinity of the tenets of Hermeneutic Constructivism with the tenets of Noaparast's (1995) position. At the core of this affinity is a recognition that Hermeneutic Constructivism overcomes the challenges that stem from the way in which Kelly (1955) dealt poorly with language. In light of this, Hermeneutic Constructivism provides a fertile and theoretically sound platform for uncovering a person's best theory or take on the world for the purpose of qualitative research. In the hope for what will spring forth from this foundation, a turn towards Hermeneutic Constructivism in application is now made. 


\section{CHAPTER 8}

\section{HERMENEUTIC CONSTRUCTIVISM: IN APPLICATION}

The previous chapters of this section have outlined and elaborated the core theoretical tenets of the position that this thesis has called Hermeneutic Constructivism. In order to promote the operationalisation of this approach for the purpose of qualitative research, this chapter develops an approach to its application that is consistent with the theoretical principles established by the Hermeneutic Constructivist position. Using the principles familiar to thematic analysis as a framework to facilitate a discussion, this chapter culminates in a consideration of the way in which the findings that emanate from the application of the Hermeneutic Constructivist approach can be presented.

\section{The Task of Hermeneutic Constructivism}

For Hermeneutic Constructivism it is important to establish a method that does not merely do the task, but is instead commensurate with what the task is. Although perhaps subtle, the difference in phrasing represents a significant divide. The do the task approach implies that we simply select from an array of existing and 'well-trodden' approaches, irrespective of the degree to which that approach is compatible with the theoretical position that underpins it. On the other hand, to start from a consideration of what the task is, will provide fertile ground from which an approach that is underpinned by the theoretical tenets of Hermeneutic Constructivism can spring forth. In light of this, a brief sketch of the core tenets of Hermeneutic Constructivism that underpin an approach to application is worthwhile. ${ }^{1}$

In terms of what the task is, Hermeneutic Constructivism represents a theoretical position to illuminate the inner outlook of the person going about the business of being human for the purposes of qualitative research. It has been established that the comportment of being human is always towards understanding and that all understanding occurs in the medium of language. In another way, language is the inextricable condition of understanding, to the extent that the Fundamental Postulate of Hermeneutic Constructivism establishes that,

\footnotetext{
${ }^{1}$ For a more elaborate explication of the principles that underlie Hermeneutic Constructivism one is directed to Chapters Six and Seven.
} 
A person's understanding processes is guided by his or her languaging of events.

The dialogue of languaging always emanates from an initial prejudice that the person projects towards a thing that is to be understood in order to illuminate it. Each prejudice carries with it a horizon that represents the possibilities or the vantage point from which the world can be understood. The horizon itself is that aspect of a person's authentic consciousness that a specific prejudice provides access to. Those aspects of authentic consciousness that the prejudice opens up toward, nourish and sustain the question and answer structure that is the ongoing dialogue of languaging.

For Hermeneutic Constructivism, the anticipatory nature of the prejudice and the way in which it illuminates an event of understanding underscores a fundamentally proactive conceptualisation of human experience. That is, Hermeneutic Constructivism takes the view that we are able to determine those aspects of the world towards which we direct our understanding processes. In addition, we are able to determine that something that we make of those events. In this movement of making something of the event, and therefore having an experience, a person is actually developing his or her prejudice(s) for future events. Thus experience itself is both conditioned by, and conditions, the prejudice. Thus, on the basis that each person selects both those aspects of the world that are to be understood, as well as the vantage point or prejudice from which they are understood, the person of Hermeneutic Constructivism is considered to be fundamentally idiosyncratic in nature.

At the core of this idiosyncrasy is the ongoing development of an inherently unique system of prejudices. Given that the prejudice represents the fundamental ontological structure of being, each of us ultimately understands the world, our self, and other people differently. In fact, because the prejudice represents the essential precondition of the comportment of being, the prejudice itself represents a significantly high order abstraction. It is for this reason that gaining an appreciation of the prejudice, and its further elucidation through authentic consciousness, represent most completely what the task is for Hermeneutic Constructivism. This recapitulation provides a foundation from which the evolution of an approach to the application of this position can originate.

\section{The Intersection of Understanding}

Hermeneutic Constructivism represents a theoretical consideration or a take on the way in which the individual person goes about the business of being human. Importantly this theoretical position is not a method per se. Instead it is a series of theoretical principles that 
should be used to inform an approach to the way that it is applied. Thus, in qualitative research that is informed by Hermeneutic Constructivist principles, the focus of consideration is the coming to an understanding of another person's prejudice(s), as the essential condition of the qualities of that person's experience. In order to facilitate the development of an approach that will enable the elucidation of another person's prejudices, a model that represents the dialogical movement of understanding that is implicit within the Hermeneutic Constructivist position will provide a valuable point of reference throughout its development. This model is titled the Intersection of Understanding. ${ }^{2}$

The Application Corollary emphasised that understanding always involves a fusion of horizons. That is, the horizon of the prejudice that one person projects towards the thing to be understood, fuses with the horizon of the thing that is to be understood. In recognition that understanding always involves interpretation, this fusion of horizons is only ever partial, perhaps reminiscent of a coalescence of horizons. The task of the Intersection of Understanding is therefore to disclose further what actually goes on within this fusion. ${ }^{3}$ In order to capture the way that these horizons coalesce with each other, a structure is needed that represents the way that these horizons can approach a unity without actually achieving that unity. Moreover, this structure should also embody the circularity of the dialogue of languaging and the historicity that sustains this dialogue. This structure is best represented by the example of the Mobius Circle.

The Mobius Circle is not unlike a three dimensional symbol for infinity $(\infty)$. The structure itself has no definite beginning or end point. It would be possible to continue to follow this structure endlessly, hence its use as a representation for the notion of infinity. Having no starting or end point, the nature of this structure not only embodies the ongoing dialogue of languaging, but also captures the way that every position within the structure is preceded by another. In so doing, the structure captures the way that at each moment in understanding we are always affected by what has been, by our time-past. As well as this, although the Mobius Circle appears to have two points along its structure that appear to achieve unity, these lines are parallel to each other and in fact never actually come into contact with each other. It is this idea that two points approach unity, without actually doing so, that captures what is meant by the notion of an Intersection of Understanding.

\footnotetext{
${ }^{2}$ The term 'intersection' is not to be confused as a slippage into a naïve realism, where the prejudice merely seeks a correspondence with the thing. Instead the notion of an intersection epitomises the way in which understanding for Hermeneutic Constructivism is always interpretation.

${ }^{3}$ Here an acknowledgement to the prolific work of Linda Viney must be made. The principles that are embedded within Viney's (1988) Mutual Orientation Model have been extolled as "the most appropriate for constructivists" (p. 191), and have here been extended.
} 
It has already been established that an interpretation can accent those aspects of the thing to be understood so effectively that the interpretation is effaced as being such, and allows the person to go on with the world. Equally an interpretation can be less effective at accenting the thing and therefore requires further development. Following the structure of the Mobius Circle, the point at which the lines appear to intersect represents the adequacy of an interpretation. That is, the point of intersection may be narrow, representing a deep understanding or an adequate interpretation. Equally, this intersection may be wider, representing a shallow understanding or a less adequate interpretation. The dialogical movement continues until such a time that the researcher develops an interpretation that accents those aspects of the participant's inner outlook so adequately that the interpretation is not seen or rather is effaced as being an interpretation at all. Instead, it is the mot juste, the most perfect words to capture the thing that is to be understood. Here the Intersection of Understanding achieves its complete elaboration. It is a symbolic representation of an ongoing dialogical movement that seeks the mot juste, as the most perfect word or phrase that encompasses the inner outlook of the person to be represented. Consistent with the notion of a mot juste however, is the never-ending possibility of there being a different or a more adequate intersection.

The Mobius Circle provides a powerful representation of the to and fro dialogue of languaging and the progressive development of our interpretations and therefore understanding. However, one may reasonably question the way that we can be assured of reaching a deep understanding of another person's experiences. In response to this, a turn is made toward the pragmatism that permeates The Psychology of Personal Constructs. Kelly (1955) suggests that, "If you do not know what is wrong with a client, ask him; he may tell you" (p. 201). ${ }^{4}$ To paraphrase Kelly, now in terms of Hermeneutic Constructivism, 'If you want to know how well your interpretation intersects with the experiences and understanding of another person, you should ask.'

\section{Case Study Design}

It is patently obvious that the focus of consideration for Hermeneutic Constructivism is the individual person - Person the Languager. The principles of the Antithetic Corollary suggest that each thesis has an inherent antithesis. Therefore, remaining consistent with this,

\footnotetext{
${ }^{4}$ Statements such as these pose a conundrum about Kelly's theoretical position especially given the way in which he dealt poorly with language and his belief that we are unable to be articulate about the way that we feel. Despite these apparent theoretical inconsistencies, the principal sentiment of Kelly's statement is comfortable within a Hermeneutic Constructivist framework. Here a person is indeed credited with the capacity to be articulate about the way in which he or she feels, and about his or her innermost qualities of experience given the inextricability of language and being theorised.
} 
the Individuality Corollary that situates the person as the foremost focus also has its antithesis in the Similarity Corollary as a consideration of how this person can also be considered similar to another person. Importantly, the Similarity Corollary suggests that a similarity in the way that a person languages his or her prejudices is not to be confused as a similarity in meaning or in experience, as per the Experience Corollary. Instead, any parallels in the way two people language their respective prejudices represents a similarity in their languaging processes. Therefore, any research design that is going to be suitable for Hermeneutic Constructivism must be able to capture both the central focus of individuality while being sympathetic to the possibilities of similarity. For this reason a series of single case studies would appear to offer a suitable approach. However as Yin (1994, 2004, 2009), a prolific writer on the subject of case study design, suggests, any case study design that contains more than a single case study is by definition a multiple case. ${ }^{5}$ Therefore, on this basis, the focus here is on the development of a modified version of the multiple case study.

Yin (2004) suggests that a key factor in the selection of a multiple case study design is its ability to demonstrate Replication Logic. That is, a capacity to identify a repetition or replication of evidence across a series of case studies. Importantly, Replication logic is not to be confused with Sampling Logic, that seeks to make statistically significant inferences from a smaller sample to an entire population. ${ }^{6}$ Instead, replication logic seeks to discover evidence to support an initial proposition. Therefore, in terms of Hermeneutic Constructivism, and when taken at face value, the replication logic of the multiple case study would provide an opportunity to illuminate the inherently idiosyncratic nature of each of the individual people included in a study.

The replication logic outlined here will allow for an emphasis to be placed upon the inherently idiosyncratic nature of being when abstracted at the level of the prejudice across a number of people. However, it is important to not lose sight of the idea that the most central consideration here is to uncover the inner outlook of the individual person. That is, discovering the prejudices that condition the qualities of a person's experience. Given that the prejudice has been emphasised as the fundamental ontological structure of being human and representative of the way that the world can come to be understood for that person, it is considered to be representative of the Self. Thus, in light of the theoretical unpacking of the Fundamental Postulate and its elaborative corollaries, the search for similarity at this level of

\footnotetext{
${ }^{5}$ Although within the field of case study research design and methodology Yin's work represents arguably the gold standard, it is the non-discipline specific nature of his work that makes it a most attractive path to follow here.

${ }^{6}$ Yin (2004) believes that the argument against the adoption of the case study is a result of a misunderstanding of these two forms of logic.
} 
abstraction is tenuous at best and represents a philosophical contradiction. ${ }^{7}$ On the basis of this, it is suggested that in order to bring the inner outlook of a person to understanding for qualitative research, a series of individual case studies that together form a modified multiple case study is necessary. ${ }^{8}$

\section{Modified Multiple Case Study Design}

Yin (2004) suggests that the rationale for adopting a single case study takes three forms. The Revelatory Case is adopted when an opportunity to explore phenomena that has been previously inaccessible to traditional scientific investigation is however accessible through a single case study. ${ }^{9}$ Secondly, the Unique Case is adopted when dealing with phenomena that are rare; and, finally, the Critical Case is selected as an opportunity to test a well-formulated theory. That is, when a theory has established a series of propositions and circumstances under which these propositions are considered correct, a critical case offers an opportunity to confirm, extend, or challenge that theory itself.

When considered in terms of Hermeneutic Constructivism, both the unique case and the revelatory case can be considered to be appropriate. By establishing that the person of Hermeneutic Constructivism is inherently idiosyncratic as a function of his or her processes of understanding, the adoption of the unique case approach follows logically. With a specific

\footnotetext{
${ }^{7}$ This thesis acknowledges that some people find it useful to make judgements about the way in which two people are considered to be similar to one another. However, at the level of abstraction with which Hermeneutic Constructivism is concerned - the Self - these comparisons are of little concern, and in fact are theoretically questionable. Despite this, if a reader finds it useful to identify similarities between the experiences of the people represented within a piece of research informed by Hermeneutic Constructivism, then it is important to appreciate that the similarity is made on the basis of an outside in view. The identification of similarity is therefore imposed upon the representations from the outside and represents a slippage into a perpetuation of the naïve assumptions about language and meaning that Hermeneutic Constructivism has sought to overcome.

${ }^{8}$ One is reminded of the words of Kastenbaum (1985), cited in Datan, Rodeheaver \& Hughes (1987), who, dissatisfied with statistical representations of groups of individuals, accuses such work of producing what he termed "Phantom Cohorts" (p. 156). Smith (1990), picking up on Kastenbaum's (1985) points, suggests that mainstream academic psychology's neglect of the idiographic level of analysis produces "indeterministic statistical zones that construct people who never were and never could be" (p. 7).

${ }^{9}$ In seeking to correct some basic misunderstandings about the representative capacity of case study research, Flyvbjerg (2004) recalls the work of Popper (1959) who suggested that the observation of single black swan is sufficient to falsify a generalisation that all swans are white. As a result, the notion of falsification is posited as one of the most rigorous tests to which a scientific proposition can be subjected. A single observation that does not fit with the proposition, renders the proposition invalid and in need of revision or rejection. It is the view of this thesis that Hermeneutic Constructivism is theoretically well situated for identifying the inherent idiosyncratic qualia of individual people and in so doing challenge the generalisations that pervade current qualitative understanding; to identify black swans as it were. In light of the in-depth approach adopted by Hermeneutic Constructivism, the words of Flyvbjerg (2004) that "what appears to be 'white' often turns out on closer examination to be "black" (p. 424) resonate with this thesis. It is for this reason that Hermeneutic Constructivism and Flyvbjerg (2004) argue that we should be cautious about overvaluing formal generalisations.
} 
focus on the initial application of the Hermeneutic Constructivist position, however, the critical case is also ostensibly valuable. That is, adopting the critical approach will provide an opportunity to confirm, extend and challenge Hermeneutic Constructivism in order to develop it further. Here, then, the unique and critical case approaches provide an opportunity to focus more deeply on the individual person, while at the same time providing an ongoing means of maintaining and developing the reflexivity of the theoretical approach. Thus a modified multiple case study that consists of any number of single case studies, or even a single case itself, is the most appropriate design for informing the application of Hermeneutic Constructivist principles. ${ }^{10}$ With this in mind, attention is directed now towards the development of a series of methods for the purpose of qualitative research. More specifically, a series of methods that are expected to facilitate the bringing to understanding of another person's prejudice(s) and the elaboration of these prejudices from his or her authentic consciousness.

\section{Self-Characterisation}

It has been established that the dialogue of languaging conditions the comportment of being toward understanding. The same to and fro movement of dialogue that allows a person to bring the world to understanding is not different when the thing to be understood is another person. In the practice of qualitative research however, the nature of the interaction is often less of a dialogue that we simply fall into or are carried along by from the outset. Instead the participants in qualitative research can expend time and energy languaging an understanding of the interview situation that may or may not be conducive to establishing a genuine dialogue. Thus, what is needed is an approach that is clear about what the purposes of the dialogue is, while at the same time, providing an opportunity for the person to find his or her voice. ${ }^{11}$ It is for this reason that a modified version of the Self-Characterisation, initially

\footnotetext{
${ }^{10}$ Smith's (2004) Interpretive Phenomenological Analysis (IPA) is one particular qualitative approach within psychology that - although ultimately succumbing to the interpretive requirements of conventional approaches to qualitative research - supports the idiographic, single case study approach. Smith (2004) suggests that a focus upon the single case has been "sorely neglected in psychology" (p. 42), a position supported by others (cf. Radley \& Chamberlain, 2001; Smith, 1993; Smith, Harre \& Van Langenhove 1995). Smith (2004) goes on to describe a focus upon the single case as an important area for development and encourages "a PhD student to be bold and consider the conduct of detailed analysis of single cases" (p. 42), "studies with an $n$ of one - doing elaborate detailed, nuanced single case studies" (p. 51), maintaining an idiographic commitment to the case which do justice to the case in its own right. Smith's (2004) position clearly supports the direction being taken by Hermeneutic Constructivism as a bold development for understanding more deeply the individual people that are the focus of consideration.

${ }^{11}$ Several authors have discussed the need for participants to be provided an opportunity to 'find their voice' in order to bring to understanding aspects of the Self (Jackson, 1988; McLeod, 1997; Androutsopoulou, 2001).
} 
proposed by Kelly (1955), provides a useful methodological vehicle for achieving these outcomes.

For Jackson (1988), a self-characterisation embodies the "spirit as well as the letter of personal construct theory" (p. 231), and is described by Neimeyer (1994) as "a quintessentially narrative technique" (p. 239). Self-characterisation provides an opportunity to illuminate the way in which we view our Self in our own words. The completed SelfCharacterisation provides what Denicolo (2003) describes as a self-disclosed written narrative that is "replete with constructs, the emergent pole at least. Not only about how people view themselves but how they perceive the worlds that they inhabit" (p. 125). ${ }^{12}$ A look at the instructions Kelly (1955) provided his clients with serves as a useful entrée to further discussion about this technique.

I want you to write a character sketch of Harry Brown, just as if he was the principle character in a play. Write it as it might be written by a friend who knew him very intimately and very sympathetically, perhaps better than anyone could really ever know him. Be sure to write it in the third person. For example start out by saying, 'Harry Brown is...' (p. 323)

Kelly (1955) believed that the use of the term 'self-characterisation' functioned to prescribe a specific structure or approach in the way that a person represented him or her self. In order to overcome this he suggests the use of the term 'character sketch', given that a sketch carries a lesser degree of formality, and as such provides a greater degree of latitude for the participant in representing him or her self. In addition, the suggestion that a participant adopt the third person perspective, as the vantage point of a good friend, provides what Kelly (1955) believes is a position from which a person can envisage him or her self in terms of a whole person. He goes on to suggest that this approach overcomes the possibility that the person will simply become introspective and present what it is that he or she anticipates that a therapist might expect, i.e. how one feels about something, one's virtues, one's faults, one's issues etc. Instead the third person perspective provides a position of safety that enables a participant to be explicit about possibly emotionally loaded issues that may have otherwise remained hidden (Androutsopoulou, 2001). As well as this, Kelly (1955) uses the term intimately to indicate that it is more than merely a superficial sketch that is sought, and uses the term

\footnotetext{
${ }^{12}$ Several others argue that the self-characterisation is a source of a person's constructs (cf. Bannister \& Fransella, 1971; Butler \& Green, 1998; Fransella, 1981; Fransella \& Dalton, 1990; Fransella \& Thomas, 1988; Hardison \& Neimeyer, 2007; Jackson, 1988; Kelly, 1955).
} 
sympathetically to help "unfreeze" (p. 324) a client from any paralysis of his or her own misgivings, allowing that person to get on with the writing of the sketch itself. ${ }^{13}$

Having established the way that one would provide instructions for the creation of a self-characterisation, Kelly further outlines the way that a therapist might undertake the analysis of such a sketch. ${ }^{14}$ Here he identified that the most important elements or a person's 'hottest' issues can be found within the first paragraph of a self-characterisation. He takes the view that the first paragraph is an indication of the way a person sees him or her self in the present, while the last paragraph is where this person envisages him or her self in the future. Kelly (1955) also identifies specific aspects to focus upon in the analysis of the selfcharacterisation: (i) observation of sequence and transition, (ii) organisation, (iii) reflection upon context, (iv) collation of terms, (v) shifting emphasis and (vi) where there is a restatement of the argument. ${ }^{15}$ He goes on to suggest that collectively, an analysis of these characteristics informs the judgments that are made about a person's self-characterisation. ${ }^{16}$ In other words, what it is that is actually being said. Kelly (1955) outlines that his principal concern is an "attempt to see the world through the eyes" (p. 329) of his clients. He goes on to suggest, therefore, that each sentence within the sketch be considered in relation to the context of the entire sketch itself. At face-value there are clear parallels between these sentiments and the principles of Hermeneutic Constructivism that warrant further consideration. However, a closer examination highlights that the application and analysis of the self-characterisation, as established by Kelly (1955), is in fact out of step with the core principles of Hermeneutic Constructivism.

Hermeneutic Constructivism was developed out of dissatisfaction with prevailing approaches to qualitative research. At the centre of this dissatisfaction is the pervasive

\footnotetext{
${ }^{13}$ Kelly (1955) acknowledges that while the statement "perhaps better than anyone could really ever know him" (p. 323), is not the most eloquent phrase, it is nonetheless a necessary inclusion. He suggests that it operates to ensure that people do not take the request in terms of how an actual friend may describe him or her, instead outlining how one views him or her self.

${ }^{14}$ As noted, the approach to the analysis of self-characterisation outlined here was first elaborated by Kelly (1955) and ultimately informs the process of characterisation analysis for Hermeneutic Constructivism. Although other approaches to self-characterisation analysis have been elaborated, perhaps most notably by Houston (1998) and Klevjer and Walker (2002), it is to Kelly's work as the original that this thesis turns.

${ }^{15}$ For a more thoroughgoing consideration of each of these approaches, see Kelly (1955) The Psychology of Personal Constructs, Vol.1., pp. 330-360.

${ }^{16}$ Crockett (1965) suggested that cognitive complexity, which is proportionate to the number of constructs that can be identified within a person's self-characterisation, is another important characteristic that is thought to be related to the complexity of a persons overall construct system. Applegate (1990) extends Crockett's (1965) idea of cognitive complexity and suggests that the "the index of abstractness captures differences in the quality of individuals' constructs" (p.213). He argues that this qualitative measure may in fact be a better measure than Crockett's simple complexity measure.
} 
adoption of a naïve and designative account of language. According to this account, words carry unambiguous or transparent meaning, and represent an assumption that is necessitated by contemporary approaches to qualitative research. Here the researcher bestows upon him or her self the interpretive authority to set down what it is that the participant - the person who actually had the experience of interest - actually means by what he or she says. Unfortunately it is this researcher derived interpretation, and not a representation of the participants themselves, that is being presented. In light of this, the Hermeneutic Constructivist position can be seen as a theoretical effort to inform the representation of real people and real lives by using the words that the person uses to bring the world to understanding and the meaning that these words hold for that person as the subject matter of focus. Thus any approach that necessitates that the researcher impose meaning upon or extract meaning out of a textual representation of another actively languaging person is out of step with the tenets of Hermeneutic Constructivism.

In light of this, the procedures for analysis of the self-characterisation as outlined by Kelly (1955), require the acceptance that an authoritative position can be achieved in order to analyse the self-characterisation. That is, to determine what the participant actually meant by: the sequencing of ideas or their organisation, including shifts in emphasis, and repetition. Each of these aspects of analysis carry a necessity for speculation, and as such represent a shift further away from the representation of the individual person. Despite this, there is still significant value in the principles inherent within the self-characterisation for Hermeneutic Constructivism. Of central value is the opportunity to provide entrée to a deeper dialogical encounter with the participant in order to discover his or her inner outlook. More specifically, the avenue that the self-characterisation provides for uncovering a person's prejudices and their subsequent elaboration from authentic consciousness. A look at how the application of the principles of the self-characterisation open up toward this dialogue when envisaged from the perspective of Hermeneutic Constructivism is valuable.

\section{Hermeneutic Constructivist Self-Characterisation}

The prejudice has been situated as the highest order abstraction with which the person of Hermeneutic Constructivism can be considered. In contrast, the construct is the highest order abstraction with which the person of Personal Construct Psychology can be considered. Therefore, in the same way that the self-characterisation sketch is, for Kelly (1955), laden with constructs, so too is the self-characterisation sketch laden with prejudices 
for Hermeneutic Constructivism. ${ }^{17}$ Recall that the prejudice conditions the quality of our inner experience, or qualia, and therefore represents the fundamental ontological structures of being. At the core of the application of Hermeneutic Constructivism is therefore an aspiration to illuminate these structures and in so doing provide a representation of the genuine inner outlook of an individual person for qualitative research. Thus, given these aspirations and the obvious value of the prejudice laden nature of the self-characterisation, it is incumbent upon the writer to provide explicit instructions for the use of self-characterisation in the context of Hermeneutic Constructivism. An example provides a useful starting point.

In seeking to understand differently the experiences of a mother named Jill Symons who has a child who was diagnosed with Down's syndrome, the following instructions may be provided concerning the completion of a self-characterisation.

I want you to write a character sketch of Jill Symons, with regard to her experience of having a child with Down's syndrome. Write it as it might be written by a friend who knew Jill very intimately and very sympathetically, perhaps better than anyone could really ever know you. Be sure to write it in the third person. For example start out by saying, 'Jill Symons is...'

The somewhat more direct nature of the instructions provided to the participant, for example the use the phrase with regard to her experience of having a child with Down's syndrome, does reduce the degree of latitude that the participant can take with this exercise. However it is anticipated that the use of the term experience without any further elaborative information, as well as maintaining the staring point Jill Symons is... will provide sufficient scope to the sketch. While providing some degree of latitude to the participant, these instructions are at the same time focused specifically upon phenomena that are of explicit interest to the qualitative research enterprise.

Androutsopoulou (2001) argues that the third person perspective provides a position of relative safety, allowing participants an opportunity to explore emotionally loaded issues that may not have surfaced for some time. Given the possibility of the surfacing of these emotions and the candour that is implicit within the self-characterisation, a researcher seeking to apply the self-characterisation does not merely post a series of instructions out to a participant and wait for its return. Instead, the researcher must avail him or her self of an

\footnotetext{
${ }^{17}$ Given the earlier argument about the way that Kelly dealt poorly with language as well as the way he situated the construct beyond language, the self-characterisation sketch is perhaps more commensurate with the position proposed here by Hermeneutic Constructivism. The theoretical unpacking of the prejudice as indissoluble from language makes the bringing of the prejudice to language in the selfcharacterisation sketch a decidedly more tenable theoretical proposition.
} 
opportunity to begin working towards an open dialogue with the participant. During this initial dialogue the researcher is able to be explicit about what is required of the person as a participant in the self-characterisation approach. The importance of this encounter should not be overlooked in order to save time. When a participant commits something to paper he or she will have a tendency to write the piece with a particular audience in mind. Therefore, the idea of this initial encounter is to provide the participant with greater knowledge of the purpose of the research. It is envisaged that with a greater understanding of the research purpose and the participant's place within the research, the participant may develop a greater sense of narrative confidence that will ultimately enhance the self-characterisation sketch that is produced.

\section{Primary Survey}

Once written, the researcher reads the self-characterisation in order to firstly gain an overall perspective on what the participant has disclosed of his or her experience. In other words, it is possible to identify quickly, in gross terms, the position with which the participant situates him or her self with regard to the experience elaborated. For example, a participant may write, Jill hates having to talk about her son or Jill takes her son's condition in her stride and has moved beyond her son's illness. Both of these statements clearly identify a gross position of difference and are perhaps a valuable position from which to begin a closer analysis. This initial overview is a movement that is not unlike a conversation that takes place between two people who have perhaps not seen each other for some time. Here one person makes a broad statement about another person followed by an invitation to learn more, for instance 'you look well...how have you been?' The goal of such a dialogue, and likewise the primary survey, is simply to gain a broad perspective of the participant's experience and to identify opportunities for exploring further.

The Primary Survey should also look for any general information. For example, it is likely that the person who completes the self-characterisation will provide some demographic material by way of getting into the flow of the narrative exercise. This information is in no way superfluous to the task at hand. In fact, a thorough Primary Survey will ensure that this information is not lost to the analysis, but can be called upon during the further dialogical unpacking of a participant's prejudices if necessary. For example, a participant might go on to emphasise the importance of his or her family. If the researcher is able to recall from an earlier aspect of the survey some of the more specific, although perhaps basic, information about the make-up of the participant's family, it may be facilitative of a more focused dialogue. 


\section{Secondary Survey}

The purpose of the Secondary Survey is to provide the core framework for entrée into a dialogue with the participant. The completion of the Secondary Survey requires that the researcher adopt a certain posture or an attitude towards the self-characterisation, a posture that is invitational in nature. ${ }^{18}$ In the conduct of the Secondary Survey the researcher is not seeking to formulate seemingly universal truths about the participant. Instead the researcher is seeking to uncover a beginning understanding of the participant that will form the basis from which the participant will be invited to illuminate something further or differently.

A Secondary Survey requires that the researcher meticulously examines the selfcharacterisation, reading each sentence independently from the broader narrative in order to identify the prejudice(s) embedded within them. It is important to acknowledge that the examination of the sketch does not involve an intuiting or the imposing of meaning upon the words of participant in the same way that contemporary approaches to qualitative research data analysis do. Instead it is a process of identifying the subject or object of the sentence. That is, reading the sentence and identifying those words that the participant has written and that signify the subject of the sentence. Here the term 'subject' is not to be confused with the person being portrayed within the self-characterisation, rather it is the topic or focus of the sentence itself. For example: a subject called Peter might have written People who know Peter would suggest that he lacks confidence. In conducting a Secondary Survey of this selfcharacterisation, one would simply identify lacks confidence as the subject of the sentence. Equally, in relation to the previous example concerning Jill Symons, in the sentence Jill hates to talk about her son, the subject would be hates to talk about son. Here the subject is considered to be synonymous with the Evident prejudice. Given that a discussion of the Evident prejudice will be provided below, it is enough to signal here that the Evident prejudice is that understanding a person can readily bring to the dialogue of languaging about the subject matter of focus. It is these Evident prejudice(s) which will ultimately provide entrée to an ongoing dialogue with the participant.

The examples above highlight the uncovering of the Evident prejudice from a sentence that firstly has a prejudice and secondly only has one prejudice. However, where a participant offers multiple prejudices within a sentence, the researcher extrapolates each

\footnotetext{
${ }^{18}$ Kelly (1964/1969) describes the notion of an invitational mood as opening up the possibility of entertaining different constructions about the same thing. He contrasts this with the indicative mood that is the setting down of the way things are either correct or incorrect. In the indicative mood the person is not open to the possibilities that something can be seen differently. The invitational mood therefore encourages a person to view a construction as a hypothesis that always has the ability to change in order to accommodate something different.
} 
Evident prejudice into a separate phrase for further dialogical illumination by the participant. The following example is a section of a genuine self-characterisation that is presented by Butler and Green (1998) and may be helpful here.

Neil is 15 -years-old and goes to King's High School. He is taking GCSE examinations in June this year. He shy and is always vulnerable and finds it difficult to communicate and get over ideas and very often is lost for words and as a result is laughed at regularly. (p. 28)

It is anticipated that the detail of the first two sentences would form a part of a thorough Primary Survey so as to be available for later use in the dialogue of languaging Neil's prejudice(s). The third sentence is, however, of significant interest to the Secondary Survey. Within this sentence the Evident prejudices of shy, vulnerable, difficult to communicate, get over ideas, often lost for words, and laughed at can be seen. Each of these Evident prejudices provides grounds for further elaboration in a future dialogue. ${ }^{19}$

\section{The Illumination of One's Prejudices}

The Antithetic Corollary emphasises, in line with the work of Hegel, that no prejudice is devoid of an inherent contradiction or antithesis. It was established that the context of the prejudice is what can meaningfully come to be understood from the horizon of that prejudice. Thus the context is that spectrum between the Evident prejudice and its antithesis as the Antithetic prejudice. The Evident prejudice has been described as the prejudice that is ready at hand, or what a person can readily envision from the horizon of that prejudice. At the other end of the spectrum, the Antithetic prejudice represents that point where a person typically evanesces toward a different prejudice in the dialogue of understanding. That is, the point where the prejudice can no longer sufficiently illuminate an understanding of the thing to be understood. As outlined previously, a person can be explicit about his or her Evident prejudice and its antithesis as the Antithetic prejudice, meaning that this person can therefore outline the context of a prejudice. Subsequently, given that the context of a prejudice determines those aspects of authentic consciousness that a person has access to, it is possible for that person to be explicit about that understanding that the prejudice provides a conduit to.

It is anticipated that the application of the self-characterisation technique, as outlined above, will provide a series of Evident prejudices that will provide entrée to a dialogue that

\footnotetext{
${ }^{19}$ It is worth noting, that the phrase get over ideas that Neil provided is not clear to the researcher, and further clarity would be sought during the elaboration phase of analysis.
} 
will ultimately lead to their elaboration. As outlined, the Evident Prejudice always has an inherent antithesis that is important to apprehend in bringing a prejudice itself to understanding. By illuminating the Antithetic prejudice it is possible to outline the context of the prejudice and subsequently the horizon of the prejudice as elaborated from that person's authentic consciousness. To explore how a person's Antithetic prejudice is uncovered and how the prejudice itself can be elaborated, Kelly's (1955) Role Construct Repertory Grid Test provides a valuable reference point. ${ }^{20}$

\section{Repertory Grid Technique}

While Kelly (1955) can lay claim to the development of qualitative forms of selfexpression through the self-characterisation, the Repertory Grid also provides a vehicle for apprehending intersubjective understanding. Although initially the uptake of the repertory grid technique was limited, the technique is today the most widely adopted aspect of Kelly's work. ${ }^{21}$ In application, the Repertory Grid enables constructs to be elicited by way of a series of elements. ${ }^{22}$ That is, the repertory grid identifies the way in which two elements are similar, and yet are also different from another. ${ }^{23}$ The resultant bi-polar constructs can then be plotted. Using a number of statistical approaches that map a person's personal construct system as well as determine the statistical relationship between each construct offers a powerful opportunity indeed. ${ }^{24}$

Although the Repertory Test can be subjected to more formal levels of analysis as outlined by Kelly (1955), for many the technique is ultimately an interview-based method. In fact Yorke (1989) believes the legacy of the Repertory Grid Technique is the provision of an agenda for ongoing and subsequent discussion. He goes on to suggest that the contextualisation by the participant of the elements that are used within the grid, offers

\footnotetext{
${ }^{20}$ This same test is commonly referred to within the literature as the Repertory Test or Repertory Grid Technique.

${ }^{21}$ Several authors who have used the Repertory Grid Technique. (cf. Aranda \& Finch, 2003; Bell, 1988, 1996, 2000a, 2000b, 2002; Bryman \& Cassell, 2006; Feixas, Bach \& Laso, 2004; Jankowicz, 2004; Kreber, 2005).

${ }^{22}$ The elements of a Repertory Grid are people identified by the participant to perform a series of roles within his or her life. These elements appear most often across the top of a grid with the corresponding constructs appearing on the sides of the grid. For a good examples of such a grid see: Jankowicz (2004) The easy guide to repertory grids, or, Fransella, Bell and Bannister (2004) A manual for repertory grid technique (2nd Ed.).

${ }^{23}$ For a thorough consideration of the nature of the construct review Chapter Three.

${ }^{24}$ The statistical method associated with the Repertory Test can be seen within the paged of Kelly's (1955) work, specifically Chapters Five and Six as well as Fransella, Bell, and Bannister (2004), A manual for repertory grid technique ( $2^{\text {nd }}$ Ed.). Moreover, the number of subsequent statistical packages for the formal consideration of the Repertory Grid Technique is testament to the ongoing utility envisaged of the method itself. For a complete review of the statistical packages available for the conduct of the Repertory Grid Technique, see Scheer (2010), Computer programmes for the analysis of Repertory Grids.
} 
potentially greater insight into that person than what Yorke (1989) describes as the "clean-cut elegance of mathematics" (p. 75). Yorke (1989) goes on to suggest that, "it is unlikely that this approach [the contextualisation of the grid elements] would yield a neat and tidy picture of the respondent, but an impressionist blur might prove more meaningful than a few superficial crisscross scratches" (p. 75). In addition, Shaw (1979), in his earlier work, dubbed the Repertory Grid Technique a 'conversational heuristic'. He suggested that the conversations that are had during the construction of the grid are of far greater importance than the formal analysis. Sharing this position, Solas (1995) suggests that what is required in the application of Repertory Grid Technique is a "means of providing a penetrating analysis of the text of grid conversations (recorded and transcribed), rather than grids per se" (p. 70). In light of the judgments of these thinkers, the unpacking of the nuances of a person's Evident and Antithetic prejudices by a surrogate statistical package represents somewhat of a philosophical disconnect with the Hermeneutic Constructivist position. Instead it is envisaged that a turn toward what Butler and Green (1998) describe as the "inventive structure of the repertory grid" (p. 37) will provide a basis for establishing an entirely conversationally based approach for setting down and elaborating a person's prejudices for qualitative research. The Self Image Profile (Butler, 1994) provides an example of an inventive approach to the Repertory Grid that is open to further modification for the purposes of Hermeneutic Constructivism.

Specifically designed for children of at least six years of age, Butler's (1994) Self Image Profile consists of a table with one column that consists of a series of self-descriptions and a six point Likert scale situated horizontally and immediately to the right of the descriptor. The pre-determined self-descriptions: friendly, confident, lazy, and shy, to name a few, make-up the column on the left-hand side. ${ }^{25}$ The participant is asked to consider these self-descriptions and to locate him or her self on a six-point Likert scale between 0 (not at all like) and 6 (very much like) in terms of the statement as I am. In other words to respond to the question: where does the participant see him or her self in terms of the self-descriptor at that present time? The participant is then asked to locate him or her self in relation to the statement as I would like to be on the same six-point scale. Importantly, in order to facilitate the rating capacities of the child, Butler (1994) only provides one pole of the construct. Although the specific purposes of his inventive approach is for making a determination of a child's self-esteem, Butler (1994) provides an example of an inventive approach to the design

\footnotetext{
${ }^{25}$ Butler and Green (1998) suggest that these pre-determined self-descriptions are the result of a significantly wide sample of children asked to comment about their own Self. Thus these predetermined aspects of the grid are more than merely arbitrary aspects for consideration.
} 
and application of the repertory grid technique. ${ }^{26}$ In so doing, he provides the impetus for a further iteration of the repertory grid technique for the purpose of unpacking a person's prejudices.

\section{The Dialogical Experience Profile}

Taking further the possibilities of an inventive approach to repertory grid application for the purposes of Hermeneutic Constructivism, a position termed the Dialogical Experience Profile (Figure 1.0) is proposed. The core principle that underpins the application of the Dialogical Experience Profile is the possibility of eliciting a person's Evident and Antithetic prejudice(s) for elaboration. Significantly, the Dialogical Experience Profile does not provide a series of pre-determined qualifiers or descriptors from which to begin a discussion in the same way that traditional repertory grids do. Instead this inventive approach uses the Evident prejudice(s) that have been discovered within the 'Hermeneutic Constructivist SelfCharacterisation Sketch'. The Evident prejudice(s) are placed at one end of a simple line scale, while at the other end of the scale is the provision for the Antithetic prejudices that will emerge through the Dialogical Experience Profile itself.

Figure 1.0: An Example of a Dialogical Experience Profile

\begin{tabular}{|c|c|c|}
\hline $\begin{array}{c}\text { Evident } \\
\text { Prejudice }\end{array}$ & Self-Report Scale & $\begin{array}{c}\text { Antithetic } \\
\text { Prejudice }\end{array}$ \\
\hline $\begin{array}{c}\text { Hates to talk } \\
\text { about her son }\end{array}$ & \multicolumn{1}{|c|}{$\begin{array}{c}\text { Able to talk } \\
\text { about her son }\end{array}$} \\
\hline $\begin{array}{c}\text { Down } \\
\text { syndrome is a } \\
\text { burden }\end{array}$ & $\longmapsto$ & $\begin{array}{c}\text { The hand we } \\
\text { were dealt }\end{array}$ \\
\hline
\end{tabular}

It has been established that a genuine dialogue or a conversation is not something that can be guaranteed in advance. Rather, a conversation is something that a person simply finds him or her self being carried along by. It is therefore ultimately in the movement of the conversation itself that the person ultimately comes to an understanding of something that is other. Thus, although the ideal context in which the Dialogical Experience Profile is to be undertaken is indeed the conversation, implicit within this advisory statement is recognition that not every interpersonal interaction is indeed a genuine conversation. In light of this, we

\footnotetext{
${ }^{26}$ Butler (2001) has identified that the Self-Image Profile has been shown to correlate significantly with the Coopersmith self-esteem scale. Despite Butler and Green's (1998) belief that the two scales measure different aspects, the mathematical power within the Self Image Profile provides a strong means of measuring a person's self-esteem. At its most rudimentary scale, self-esteem is a consideration of the difference between 'as I am' and 'as I would like to be' ratings.
} 
should not simply rush into the application of the Dialogical Experience Profile on the basis that it will automatically open up a conversation. Rather, it is suggested that the researcher inform the participant that his or her self-characterisation has provided the initial basis for further discussion. A statement that resembles the following is perhaps a useful entrée to the Dialogical Experience Profile.

I firstly want to thank you for taking the time to complete the self characterisation in the way that you have. You have provided some invaluable beginning insight into your experiences. I am most interested today in exploring further what these statements actually mean to you and the way in which you would explain them to someone. Equally as important for me is to get a sense of those aspects of your experience that these statements do not relate to. In other words, the opposites of the statements you provided. So, for each statement I am going to ask you to think about the following question: 'If you were not...then what would you be?' Then I am going to ask you to rate yourself on this scale between the statement you provided in the self-characterisation and the opposite you identified today in terms of where you believe yourself to be in relation to your experiences, and then to identify where you would like to be. During this process I might need to ask several questions so I can understand as much as possible about your unique experiences.

Beginning with the first Evident prejudice on the Dialogical Experience Profile, the researcher would ask the participant to do one of two things; either elaborate what is meant by the Evident prejudice or to think in terms of If you were not (the 'evident prejudice') what would you be. The aim of this latter question is to uncover the Antithetic prejudice. Recall that the context of the prejudice is that spectrum between the Evident prejudice and the Antithetic prejudice. Moreover, although the Antithetic prejudice represents the limit of the prejudice itself, it is still part of the context of the prejudice and can therefore be meaningfully brought to language. ${ }^{27}$ Importantly, the decision by the researcher to choose one approach to questioning over another is merely a means of avoiding repetition and to encourage a more conversational tone to the dialogue.

\footnotetext{
${ }^{27}$ Hermeneutic Constructivism takes the Antithetic prejudice as that position along the spectrum where one can no longer be articulate about his or her Evident prejudice. It is this point where the illuminating power of that prejudice subsides and typically evanesces to a different prejudice.
} 
Throughout the dialogue it may become apparent that a person's Evident prejudice requires further elaboration when viewed in the light of the Antithetic prejudice. Also, by following the theoretical unpacking of the Organisation corollary, the understanding that a researcher develops of a participant's prejudice(s) continues to develop as subsequent prejudices are illuminated further. Thus the Dialogical Experience Profile is itself an ongoing dialogue within which a researcher develops an understanding of the structures that condition the qualities of another person's inner experience. In light of this, the opportunity for ongoing movement through the intersection of understanding with the participant is an opportunity indeed and one not to be prematurely hastened. ${ }^{28}$

The next phase of the Dialogical Experience Profile is to determine where the participant situates him or her self on the spectrum between the Evident prejudice(s) and Antithetic prejudice(s) with regard to experience. The identification of this position is not simply arbitrary. Instead it is a genuine representation of the present Self as that point within the context of a prejudice that a person is situated at that present time. In other words, it represents the way that a person understands his or her experience in the here and now of being. Also it is recognition of the way that the person of Hermeneutic Constructivism can bring his or her experience to understanding in different ways at different moments in time. In following this inherently proactive conceptualisation of the person, the participant is asked to also indicate where, on the same spectrum between the Evident and Antithetic prejudice, the ideal position upon this continuum is for you.

Having identified both the present and ideal position, the participant is asked to consider the following question, what do you believe would be required for that to happen? In other words, what would be required for the participant to envision the world from a different position within the horizon of his or her prejudice. It is expected that the responses provided to this question will inform the developing understanding of both the participant's Evident and Antithetic prejudice(s). This is because, in order to provide a response to this question, the participant must engage with those aspects of his or her authentic consciousness that the prejudice provides access to. It is important to appreciate at this point the possibility that the participant may not have previously had to contemplate the possibilities of an alternative way of looking at the world beyond the vantage point of his or her Evident prejudice. Thus, the participant's being asked to contemplate, and to engage with aspects of his or her Antithetic prejudice, may perhaps be for the first time. More specifically, being

\footnotetext{
${ }^{28}$ It would be of significant utility to use 'pen and paper' for notes during this phase of the Dialogical Experience Profile so that a brief sketch of the researcher's developing understanding of each construct can be mapped out. The paper and pen provides an invitational space to which both participant and researcher can contribute in the development of shared meaning and understanding.
} 
asked to contemplate the possibilities of envisaging the world anew from alternative positions, is a process that is potentially confronting. What makes this process confronting is that the dialogue of languaging that a participant carries out in contemplating the possibilities of an alternate position, and therefore anticipating what would be required in order to get there, involves an engagement with higher order aspects of his or her prejudice system and subsequent authentic consciousness. Despite this caution, the possibility of a participant engaging with and amplifying aspects of his or her greater system of prejudices and subsequent authentic consciousness, makes this contemplation a valuable approach indeed.

Despite the value for understanding a participant more deeply, this contemplation of higher order aspects of a person's prejudice system and concomitant aspects of authentic consciousness, is not a capacity to be used flippantly. The movement of the Dialogical Experience Profile facilitates the contemplation of perhaps less routinely accessed aspects of a person's greater system of prejudices. For this reason, it would not be the recommended practice to ask, what is required for you to move from here? with each prejudice that is identified. More specifically, it is not typically recommended that a researcher ask this question for prejudices where the participant has identified his or her present position to be diametrically opposed from the position that this same person wishes to be. In other words, prejudices where a participant has identified his or her 'where I would like to be position' at the opposite end of the spectrum to the 'where I am now' position (Figure 2.0) are anticipated to pose the greatest degree of confrontation to the participant.

For Hermeneutic Constructivism, the diametrically opposed nature of the ideal position from the where I am now position is confronting for a participant because it is believed to represents a loss. For a person to be able to identify the where I would like to be position indicates that at some stage he or she has engaged in a dialogue of languaging about the possibilities of his or her ideal position. Thus, to identify the where I would like to be as a possibility, and yet identify the where I am now position as significantly different, represents a loss of what has previously been considered as possible. In line with this, given that a prejudice system represents the possibilities of understanding that are available to a person, and therefore represents the Self, the loss of a possibility of envisaging the world represents a loss of an element of the Self. Although it could be said that a loss is represented in all situations where a participant identifies the present position as different from his or her ideal position, it is not considered to represent the same degree of loss as in a circumstance where these positions are diametrically opposed. What can be made of this, then, is that the requirement for a participant to confront this loss and to elaborate it further is beyond the scope established here of Hermeneutic Constructivism. Hence, from this consideration 
develops a general rule to guide the application of the Dialogical Experience Profile. That is, in order to forestall the possibility of a participant being confronted by aspects of his or her loss, only regarding those prejudices where the participant locates his or her where I am now and where I would like to be within the same half of the scale between the Evident and Antithetic prejudice, should the researcher ask the question, what is required for that to happen? An illustrative example how to identify which prejudice is considered appropriate to pursue this question can be seen in Figure 2.0.

Figure 2.0: When to Ask 'What is required for that to happen?'

\begin{tabular}{|c|c|c|}
\hline $\begin{array}{c}\text { Evident } \\
\text { Prejudice }\end{array}$ & Self-Report Scale & $\begin{array}{c}\text { Antithetic } \\
\text { Prejudice }\end{array}$ \\
\hline $\begin{array}{c}\text { Hates to talk } \\
\text { about her son }\end{array}$ & & $\begin{array}{c}\text { Able to talk } \\
\text { about her son }\end{array}$ \\
\hline $\begin{array}{c}\text { Down } \\
\text { syndrome is a } \\
\text { burden }\end{array}$ & 1 & There dealt \\
\hline
\end{tabular}

Note: $\odot=$ Where I am now $\bullet=$ Where I would like to be

Notice the diametrically opposed nature of the 'where I am now' from the 'where I would like to be' in first prejudice. It would not be recommended practice to pursue the 'what is required for that to happen' question with this prejudice. On the hand, the proximal nature of the 'where I am now' from the 'where I would like to be' in the second prejudice, supports the posing of this question.

In order to support the faithful application of the theoretical principles of Hermeneutic Constructivism that inform the Dialogical Experience Profile, the key principles that have been outlined thus far are restated: (a) let the Evident prejudice form one side of the Dialogical Experience Profile; (b) the researcher should ask the participant to language his or her Antithetic prejudice by way of the question If you were not (the 'evident prejudice') what would you be; (c) through the dialogue of languaging that follows the model of the Intersection of Understanding, the researcher restates those aspects of the participants prejudice(s) and subsequent authentic consciousness that have already been garnered, for substantiation by the participant. Here if the researcher wants to know how well an interpretation intersects with the understanding of another person, he or she should ask, prior to shifting the focus of the dialogue to a subsequent prejudice that stands in need of elaboration; ${ }^{29}$ (d) the researcher asks the participant to locate where along the spectrum

\footnotetext{
${ }^{29}$ Yorke (2001) raises doubts about the technical and conceptual qualities of the repertory grid. He argues that the complexity of bipolar rating scales raises questions about the conceptual quality of the scales from a linguistic perspective. He goes on to cite several works that challenge the assumptions that are embedded within bipolar models including those of Kelly. He suggests that analyses conducted upon bipolar scales are potentially problematic given their ambiguity as well as their inherent and unavoidably linguistic nature of the construct poles. This ambiguity is supported in kind by the work of Hagans, Neimeyer and Goodholm (2000) who suggest that the method of construct
} 
between the Evident prejudice and Antithetic prejudice that he or she would be located with regard to his or her experience. The researcher should then follow up with the simple question why? This provides an opportunity for the participant to elaborate further the Evident and Antithetic prejudice; (e) the researcher should ask the participant where upon this spectrum would be his or her ideal position. Once again the question why? should be used to seek elaboration. Finally, (f) in prejudice(s) where the participant has identified his or her ideal position within the same half of the Dialogical Experience Profile scale as his or her present position, the researcher should seek to elaborate what might be required for the participant to envision a shift to that ideal position. In so doing, the movement of the Dialogical Experience Profile may illuminate higher order aspects of the participant's greater system of prejudices and their associated aspects of authentic consciousness.

\section{Laddering}

For Hermeneutic Constructivism, the Organisation Corollary provides the theoretical groundwork for the way in which each prejudice is situated within a greater system of prejudices. It has been established that each prejudice evanesces into another as a function of the dialogue of languaging. The evanescent movement from one prejudice to another carries an inherent consistency that preserves a degree of lawfulness within the prejudice system.

This tendency to consistency means that it is possible for the person envisaged by Hermeneutic Constructivism, through the dialogue of languaging, to progressively move through his or her system of prejudices and to be articulate about that movement. This does not mean that this same person could bring his or her entire system of prejudices to language, because it is inconceivable to comprehend how a person could pose every question necessary in order to access each prejudice within his or her greater system of prejudices. Instead, it means that in the to and fro dialogue of languaging that essentially takes the form of question

elicitation has implications for the final construct presented. Comparing Kelly's (1955) 'Triadic Difference Model' and Epting, Suchman and Nickeson's (1971) 'Triadic Opposite' method these authors underscored some significant differences in the elicited construct. In providing a recommendation for overcoming what Yorke (2001) considers to be "an unavoidable wrestle with words and numbers" (p. 184), he goes on to suggests that what is needed is an opportunity for the person in question to elaborate the meanings of his or her construct poles. He also suggests that onthe-spot productions of repertory grids by respondents are at risk of the same drawbacks as over hasty scale production itself. In fact, he seeks to remind researchers that "the day-to-day real world is untidy and riddled with inconsistency, ambiguity and hiddenness" (p. 184) and that these characteristics are to often forgotten. Instead, Yorke (2001) suggests we tend to delight in the apparent 'clear-cut' and precise nature of methodologies like the repertory grid. Yorke's (2001) critique provides inadvertent support for the application of Hermeneutic Constructivism. The Dialogical Experience Profile provides an opportunity through the self-characterisation sketch for the participant to illuminate his or her Evident prejudice and, through the ongoing dialogue of the profile itself, the Antithetic prejudice and all that they embody is elaborated. Although the Dialogical Experience Profile has not been outlined as a statistical application, it is nonetheless considered to be sufficiently flexible to apprehend the untidy day-to-day inconsistencies of being human that Yorke (2001) makes note of. 
and answer, a person can move through those aspects of his or her prejudice system that these questions open up toward. Thus, in order to capitalise on this, a method of application that is able to facilitate this movement is necessary. A consideration of the notion of Laddering, an approach first elaborated by Hinkle (1965) in his unpublished doctoral dissertation is worthwhile.

Undertaken initially as a test of Kelly's original Organization Corollary, Hinkle (1965) elaborated what he described as the hierarchical technique for eliciting the superordinate constructs of the preferred self hierarchy. Now most commonly referred to as laddering, the technique offers an opportunity to explore the constructs that a person places greater importance upon. ${ }^{30}$ It is suggested that in coming to understand these higher order structures it is possible to reveal more about the person's construing processes and subsequently his or her processes of personal meaning-making. Although debate concerning the technique's utility has a history dating back to its inception with Hinkle (1965), ${ }^{31}$ at its core the process of laddering is considered to provide an opportunity to elicit the superordinate constructs within a person's greater construction system. ${ }^{32}$

According to Fransella (2003), a leading figure in Personal Construct Psychology and more specifically in the application of the laddering technique, "it is within the process of laddering that one gets nearest to that experience of being almost a part of the other person" (p. 113). For Rowe (2003), core constructs are concerned with notions of survival and thus have a greater degree of stability, while Butler (2006) suggests that a person's higher order or more core constructs "lie fundamentally at the heart of the individual's sense of self, guiding each anticipatory choice they make" (p. 27). It stands to reason, then, that given the influence of these core structures upon the way in which the person comes to understand the world, the illumination of these higher order structures is invaluable in understanding that person.

Although there are no formal instructions for the process itself, laddering follows a form of recursive questioning in order to elicit higher order constructs (Neimeyer, 2009; Neimeyer, Anderson \& Stockton, 2001). The technique requires that a person ask the

\footnotetext{
${ }^{30}$ For Neimeyer (1993a) ladering provides an opportunity to explore not only a person's superordinate constructs, but also the placement of a person's preferred self.

${ }^{31}$ Landfield and Epting (1987) for example, argue that the movement down a person's construct system by way of a technique called 'pyramiding' offers greater utility than laddering alone. Despite this, Butt (1995) notes during his use of laddering technique that "what did not happen was systematic ascending of a hierarchical construct system" (p. 230).

${ }^{32}$ For Leitner and Thomas (2003) the possibility of a person experiencing a profound threat from the invalidation of his or her core constructs somehow places these core elements of a supposedly low level of awareness. The limited conscious awareness of the more core elements of our construct system is a position shared by McWilliams (2004), who argues that we seeks to validate or avoid invalidation of our core structures.
} 
participant to identify a preferred end or pole of a bipolar construct and then ask for the participant to identify what is important for you about that pole as opposed to the other? The participant answers, and the construct that is supposedly embedded within that answer now becomes the topic of consideration. Having identified the embedded construct, the question what is important for you about...? is again posed. This question is often followed again and again by the open question whereas...? , in a process that constantly seeks the opposite. ${ }^{33}$ The following passage from Neimeyer (2009) effectively captures this movement.

The therapist continues in this way, inquiring about a preference, a reason or advantage, and its contrast in a cyclical pattern of questioning until the client begins repeating responses or finds it difficult to formulate a further construct. (p. 36)

It is believed that through this movement it is possible to effectively climb the ladder of a person's construct system and identify progressively more superordinate constructs of that system.

The reputed power of the laddering technique is captured through the words of Costigan, Closs and Eustace (2000), who, citing Rowe (1978), emphasise that laddering is not a party game. These authors go on to suggest that one must be cognisant that exposing an individual to an element of his or her own construing that is otherwise hidden can be quite confronting psychologically. ${ }^{34}$ In light of this potential, the practical caveats concerning the technique of laddering expressed by Fransella (2003) are useful for noting: (a) no more than three subordinate constructs should be laddered $;^{35}$ (b) one should only ever ladder the preferred pole of the construct given the risk of following unknown - and potentially psychologically confronting - pathways by pursuing the non-preferred side; ${ }^{36}$ and (c) at the first sign of unease, or of the participant disclosing such constructs as being alive or purpose on earth, it is a reasonable expectation that the top of that person's construct system has been achieved, and that the process should cease. Despite the value of the principles of laddering to the Hermeneutic Constructivist position, it is perhaps worthwhile reiterating at this point

\footnotetext{
${ }^{33}$ Fransella (1995) suggests that laddering "is no more and no less than asking the question why?" (p. 142). Similarly, Neimeyer, Anderson and Stockton (2001) believe the phrase "what is the advantage of that?" is equally as valuable as the 'why' question in the process of laddering.

${ }^{34}$ Butler (2006) contends that laddering is, for many practitioners, potentially the most powerful procedure for eliciting the values that a person holds and the meaning with which that person organises his or her world. In line with this, Fransella (1995) suggests that with 'laddering' it is possible to get directly into a person's basic system of values, which can be a "far from peaceful business" (p. 143). ${ }^{35}$ Interestingly, Costigan, Closs and Eustace (2000) have identified the successful laddering of five constructs within their work.

${ }^{36}$ According to Jankowicz (1999), in email correspondence with Costigan, Closs and Eustace (2000), we should always follow the implicit pole of the construct in laddering.
} 
that the way that Kelly (1955) situated the construct outside of language makes the movement of laddering theoretically difficult to sustain. In other words, if the construct cannot come to language, then what is it that is being laddered?

\section{Hermeneutic Constructivist Laddering}

For Hermeneutic Constructivism, however, the prejudice represents the fundamental ontological structure that conditions the human comportment toward understanding. In addition, it is a structure that is itself inextricable from the medium of language and can therefore be clearly articulated. Thus the prejudice is the highest order abstraction according to which the person of Hermeneutic Constructivism can be considered. The opportunity to illuminate and progressively move through a person's system of the structures that condition his or her inner qualities of experience is an opportunity indeed. Thus the possibility of illuminating a person's higher order prejudice(s), and therefore gaining a glimpse at the Self, reinstates Fransella's (2003) reminder that the greatest respect be bestowed upon the power of laddering as a research tool.

Upon the advice provided by Fransella (2003) that only three prejudices are selected for laddering, attention is directed toward a consideration of which three prejudices are likely to be appropriate. As mentioned previously, Kelly (1955) suggests that the first paragraph of a self-characterisation may be viewed as if it were a key statement about the person's view of his or her self in the present or, as Androutsopoulou (2001) suggests, as containing the "hottest issues" (p. 80). Kelly (1955) also suggests that the last paragraph of a selfcharacterisation provides an indication of a person's perceived outlook for the future. ${ }^{37} \mathrm{In}$ light of this, two prejudices from the first paragraph and one from the final paragraph should - where practicable - be used as the basis for the application of laddering from the perspective of Hermeneutic Constructivism.

As described previously, a participant can of course provide several prejudices within a sentence, not to mention a paragraph. Thus the issue of deciding which prejudice(s) are to be elaborated stands in need of further consideration. The work of Fransella (2003), amongst others, when considered in terms of Hermeneutic Constructivism, would suggest that elaboration of the Evident prejudice is a fruitful avenue to pursue. ${ }^{38}$ However, translating the position proposed by Jankowicz (1999) once again into terms consistent with Hermeneutic Constructivism, the Antithetic prejudice would be suggested to provide the greatest value. In

\footnotetext{
${ }^{37}$ This reading of Kelly's work is also shared by Denicolo (2003), as well as by Fransella and Dalton (1990).

${ }^{38}$ Fransella (2003) and other authors suggest the Preferred Pole of the construct as the avenue to pursue for the application of the laddering technique from a Personal Construct Psychology perspective.
} 
light of this conjecture, the decision of which prejudices to elaborate is based upon two key assumptions: (i) that the laddering of a particular prejudice will provide an opportunity to illuminate higher order prejudices and subsequent aspects of authentic consciousness, and (ii), perhaps most importantly, that the participant is not likely to be unduly confronted by the progressively higher order movement of the laddering technique.

With these principles in mind, it is anticipated that the laddering of the Evident prejudice(s), where the participant locates their ideal position within the same half of the Dialogical Experience Profile as their present position, is less likely to be confrontational for the participant given the proximity of his or her ideal and present positions. Moreover, one will recall that because the Evident prejudice is ready at hand, it is more likely to have previously come to the fore in the dialogue of languaging. In other words, it is more likely that a person will have previously envisaged the world from the vantage point provided by his or her Evident prejudice. On the other hand, the Antithetic prejudice itself may not have previously come to understanding. Thus having to envisage the world - perhaps for the first time - from the vantage point that the Antithetic prejudice provides, may indeed be confronting for the participant. For this reason it is suggested that the Evident prejudice provides a more appropriate avenue to pursue in the process of laddering. An illustrative example of this can be seen in Figure 3.0.

Figure 3.0: Selecting an Appropriate Prejudice for Laddering

\begin{tabular}{|c|c|c|}
\hline $\begin{array}{c}\text { Evident } \\
\text { Prejudice }\end{array}$ & \multicolumn{1}{|c|}{ Self-Report Scale } & $\begin{array}{c}\text { Antithetic } \\
\text { Prejudice }\end{array}$ \\
\hline $\begin{array}{c}\text { Hates to talk } \\
\text { about her son }\end{array}$ & & $\begin{array}{c}\text { Able to talk } \\
\text { about her son }\end{array}$ \\
\hline $\begin{array}{c}\text { Down } \\
\text { syndrome is a } \\
\text { burden }\end{array}$ & 1 & $\begin{array}{c}\text { The hand we } \\
\text { were dealt }\end{array}$ \\
\hline
\end{tabular}

Note: $\odot=$ Present Position $\boldsymbol{0}=$ Ideal Position

In the first prejudice the participant has identified his or her ideal position in the other half of the selfreport scale to the present position. In this case the Evident prejudice 'hates to talk about her son' would not be appropriate. In contrast, in the second prejudice the participant has identified both her ideal and her present positions within the same half of the self-report scale, making the Evident prejudice 'Down syndrome is a burden' an appropriate avenue for laddering.

The application of the laddering technique within Hermeneutic Constructivism is intended to yield higher order prejudices and illuminate subsequent aspects of a participant's authentic consciousness. From the position of an Evident prejudice, the researcher asks the participant Can you tell me why (the evident prejudice) is important for you. Here the answer provided by the participant is an elaboration of those aspects of authentic consciousness that 
the Evident prejudice illuminates. In response to the answer provided, the participant is then asked; (i) why do you believe that is important, or (ii) if it wasn't that what would it be. In both of these example questions, the that is the subsequent prejudices that are embedded within the elaboration that the participant has provided of his or her Evident prejudice. Recall that the embedded nature of the prejudice is not a slippage into a requirement for intuiting or guessing what the prejudice is on the part of the researcher. Instead, the prejudice is the subject of the elaboration provided.

Throughout the dialogue of laddering, a researcher must remain vigilant for any signs of unease that may be an indication of the abstraction of prejudices at a particularly high level within that person's system of prejudices. It is also possible that prejudices that have already been illuminated, through the self-characterisation sketch and the Dialogical Experience Profile, are already of significantly high order. In this case it is anticipated that the participant may find it difficult to move beyond them. In line with this, the integral nature of the prejudice system means that it is theoretically possible for a person to ladder different Evident prejudices to a consistent higher order prejudice. ${ }^{39}$ It is important to recognise that despite these theoretical possibilities it is not the intention here to apply the laddering technique in an effort to merely provide a map of a person's prejudices. Instead the dialogical movement of laddering provides an opportunity to come to an understanding of the experience of phenomena for another person at a particularly high level of abstraction. That is, an understanding of the person at the level of the structures that condition his or her innermost qualities of experience, the structures that provide the very possibility of having qualia at all. $^{40}$

\footnotetext{
${ }^{39}$ Rowe (2003) found, much to her surprise, that the number of core constructs that a person has is limited and that these constructs tend to have a degree of universality or persistence for that individual person.

${ }^{40}$ Here is an opportune moment to mention the growing body of literature concerned with the similarities between the qualitative research interview and a therapeutic endeavour. In fact, the dialogical aspects of qualitative research and therapeutic discourse alike lead McNamee (1994) to suggest that she does not "see the research endeavour as differing substantially from a therapeutic interview" (p. 70). However, contrary to this position, Brinkman and Kvale (2005) contend that seeking change in a person - in the therapeutic sense - as opposed to knowledge of the participant - in the research sense, is an unethical enterprise. It is worthwhile noting that the first and foremost consideration of Hermeneutic Constructivism is with a deeper more nuanced understanding of the individual person and not with change in that person. Despite this, the possibility that Hermeneutic Constructivism might open up the possibilities for change is exciting indeed. The dialogue that is the Dialogical Experience Profile - specifically the elaboration of the context of the prejudice - provides a participant with an opportunity to understand the possibilities that he or she has available for envisaging the world differently. If a participant in the research enterprise finds it beneficial to contemplate - even for a moment - the possibility of envisioning his or her world anew, Hermeneutic Constructivism has indeed exceeded its focus. Indeed, such an approach represents a positive frontier for qualitative research.
} 
To ensure that sufficient clarity is provided to the application of laddering in a Hermeneutic Constructivist framework, key principles are re-stated here: (a) where possible the researcher selects two prejudices from the first paragraph and one from the last paragraph of the self-characterisation to be laddered; (b) of those prejudices, the researcher should only seek to elaborate the Evident prejudice with which the participant has identified a degree of consistency between his or her self-report of present position and ideal position; (c) the researcher should ask the question why is that important?, or If it wasn 't that what would it $b e ?$, in an effort to maintain a dialogical movement to the laddering of the participant's system of prejudices; (d) the researcher should always be vigilant concerning the level of a participant's prejudices being considered and the ease of the participant, given that they are indicators of where to stop the laddering process; and (e) the researcher should remember that the earlier techniques - self-characterisation sketch and the Dialogical Experience Profile may have already provided some higher order prejudices from which an even higher position cannot be obtained by or is rather difficult to obtain for the participant.

\section{Presenting the Inner-Outlook.}

By now it has been clearly established that the focus of consideration for Hermeneutic Constructivism is the 'inner outlook' of the individual person going about the business of being human for the purposes of qualitative research. Hermeneutic Constructivism is concerned to represent this person at a particularly high level of abstraction, at the level of the structures that condition the qualities of internal experience. However, convention concerning the presentation of experiential data in qualitative research more broadly influences what it is that a reader expects to see here in the presentation of this 'inner outlook'. Thus the fundamentally idiographic focus of any research that is informed by Hermeneutic Constructivism stands in stark contrast to representations that are informed by contemporary approaches to qualitative research, approaches that ultimately seek superficial generality between the real people and real lives encountered and in so doing zoom out from the elements of idiosyncrasy. It follows, therefore, that a different means of presenting this inner outlook should be anticipated. Nevertheless, in order to establish how the presentation of this inner outlook might well unfold, landmarks that are familiar to the presentation of contemporary qualitative research provide a useful backdrop for making determinations of the developments made by Hermeneutic Constructivism. 
Within the field of qualitative research, it is the work of Colaizzi (1978) that is most commonly used a framework for the analysis of qualitative data. ${ }^{41}$ This framework consists of six specific steps that are familiar to the practice of qualitative research. These steps provide a useful framework for highlighting the developments that have been made by Hermeneutic Constructivist position. Each of Colaizzi's (1978) six steps will thus be outlined, followed by a discussion of how Hermeneutic Constructivism can be seen to overcome the naïve assumptions about language that are embodied by each of these steps.

\section{STEP 1 - READING THE TRANSCRIPT AND OBTAINING A SENSE OF THE WHOLE.}

The principal consideration here is that from the perspective of Hermeneutic Constructivism the understanding that is achieved of the person comes from the dialogical movement between the researcher and the participant. This understanding does not arise from the intuitive processes of the researcher seeking to make something of a dialogue that has been reduced to text as a transcript. Instead the way in which Hermeneutic Constructivism has rehabilitated the centrality of dialogue in genuine understanding means that the greatest proportion of the analysis of the subject matter of focus - the individual person - has already been undertaken at the conclusion of the Dialogical Experience Profile.

As described previously, the conventional process of qualitative data analysis - and subsequently the process that is also proposed by Colaizzi (1978) - begins by concretising the dialogue that transpired during the research interview into a verbatim account or text as a typed transcription. These transcriptions provide the basis for later, and further, analysis by the researcher. As the beginning phase of analysis, the researcher reads the transcripts and supposedly attains a sense of the whole. In contrast to this, the inner outlook of the person that Hermeneutic Constructivism envisages is to the greatest extent present within the Dialogical Experience Profile itself. Here the use of the phrase to the greatest extent is consistent with the Temporal Distance Corollary that establishes that a person always has the possibility of understanding a thing further or differently.

Given that the level of abstraction of the prejudice is sufficiently high - the level of the Self-seeking to determine what it is that a participant actually means by his or her prejudices, in excess of what he or she has already elaborated in the dialogical encounter itself, represents a philosophical faux pas. Therefore, to reduce this dialogue to merely a representation in the form of a verbatim transcription is considered to be a reduction indeed.

\footnotetext{
${ }^{41}$ Colaizzi (1978) was selected in light of the popularity of his work. According to Shin, Kim and Chung (2009), Colaizzi and van Mannen are the most frequently quoted and adopted theoreticians in the qualitative research arena.
} 
Instead it is suggested that a more contemporary and somewhat more representative means of capturing this dialogue is the use of a digital audio recording. ${ }^{42}$ Captured in this way, the recording lends itself to future and further immersive engagement with the material that is still filled with many of the qualities and vibrations of the encounter itself. Such a recording provides an opportunity to re-listen to the various intersections of understanding. After all, it is these points of intersection that embody the understanding of each prejudice and the subsequent aspects of authentic consciousness that was achieved in the dialogical encounter itself. Importantly, in re-listening to the recording, a researcher is not seeking to add or intuit meaning to these intersections of understanding. Instead he or she uses the recording as a means of highlighting further those aspects of a participant's experience that have been substantiated by the participant within the intersection itself.

\section{STEP 2 - EXTRACTION OF SIGNIFICANT STATEMENTS AND PHRASES PERTAINING TO THE} PHENOMENA.

Convention would suggest that a researcher scan verbatim transcripts and identify those aspects that he or she considers to be significant statements and phrases that pertain to the participant's experience, or perhaps those that ring bells with the words of other subjects. In contrast, for Hermeneutic Constructivism, a researcher reviews the Self-Characterisation sketch sentence by sentence in order to extract the subject or object of the sentence. As outlined previously, this is not an intuiting of what is meant by the sentence itself. Instead the identification of the object or subject of the sentence represents the Evident prejudice. It is this Evident prejudice that provides entrée to the Dialogical Experience Profile and the subsequent movement toward the intersections of understanding. Through the Dialogical Experience Profile it is the participant who elaborates his or her Evident prejudice, by contemplating the Antithetic prejudice as well as those aspects of authentic consciousness that the prejudice itself provides access to. Thus here the need for the researcher to identify those aspects of another actively languaging person's experience that are supposedly significant while independent of that person is made redundant.

\section{STEP 3 - TO FORMULATE MEANINGS FROM THE SIGNIFICANT STATEMENTS AND PHRASES.}

Qualitative research requires that a return be made to those statements and phrases that the researcher identified as significant, and to make meaning of them. That is, to identify what it was that the participant actually meant by the significant statements that the researcher

\footnotetext{
${ }^{42}$ Most qualitative researchers already produce a recording of the interview that is ultimately the raw material of the interview itself. This is then transcribed.
} 
identified. In contrast, the Dialogical Experience Profile provides a vehicle through which the prejudice and its subsequent elaboration from authentic consciousness can be brought to understanding. By following the model of the Intersection of Understanding, the dialogical movement of languaging is able to continue without limits. Much like a genuine dialogue or conversation, the movement of languaging can continue until such a time that the participant confirms the researcher's understanding of each prejudice and its elaboration as adequate.

The confirmation of the understanding that is achieved by the researcher is represented by the model of the intersection of understanding. More specifically, this intersection represents a fusion of horizons. Thus, in the dialogue of the Dialogical Experience Profile itself, the horizon of the researcher coalesces with the horizon of the participant, and, through the continued movement of languaging, the fusion of these horizons develops until the participant acknowledges the adequacy of the researcher's understanding. ${ }^{43}$ Once again this rehabilitation of the centrality of the dialogic is at odds with Colaizzi's (1978) notion of selecting significant statements and formulating meaning while removed from any dialogical opportunity with the participant.

\section{STEP 4 - CLUSTERING THE MEANING INTO THEMES.}

Conventionally the researcher takes the meanings that he or she has applied to or intuited from statements identified as significant and uses them to locate areas of similarity across participants within a study. Here the meanings that are identified by the researcher as being similar are clustered together to form an aggregate meaning. These aggregate meanings are given a broad or umbrella label and become a theme that is somehow encompassing of each of the meaning units that it represents and subsequently each of the participants.

This notion of clustering meaning across people in order to develop a theme is in fact a search for generalisation. However, as outlined previously, the idea that a theme can embody the inherently idiosyncratic inner outlook of one person, let alone several people, is incontrovertibly at odds with the theoretical foundations of Hermeneutic Constructivism. The Similarity Corollary holds that 'To the extent that one person brings to language his or her system of prejudices in a manner comparable to that of another, his or her languaging processes can be considered similar to that of another person'. The theoretical unpacking of this corollary establishes that similarity between the prejudices of two people does not simply represent a similarity in meaning, or for that matter a similarity in experience. Instead it represents a similarity in the understanding processes of these people, as outlined through the

\footnotetext{
${ }^{43}$ The model of the Mobius Circle elaborated previously embodies this movement.
} 
Experience Corollary. As well as this, it has been established that the prejudice conditions our understanding processes, and is therefore synonymous with the Self. This makes the search for commonality across the prejudices of different people theoretically inconsistent with Hermeneutic Constructivism. ${ }^{44}$

STEP 5 - INTEGRATE RESULTS INTO AN EXHAUSTIVE DESCRIPTION OF THE PHENOMENA.

Colaizzi (1978) suggests that a researcher should generate a statement that brings together the meaning units that he or she has situated under each theme. This statement, as Colaizzi (1978) notes, should be considered exhaustive in its apprehension of the phenomena of interest and the individual(s) represented. For Hermeneutic Constructivism, the phenomenon of interest is the inner outlook of the person going about the business of being human at the level of the prejudice. It is anticipated that the application of the Dialogical Experience Profile, as well as the use of the laddering technique, provide a vehicle for increasing the depth of understanding that is achieved of a person. Despite this, the finitude of human understanding is such that the notion of an exhaustive statement or one that is fully comprehensive is theoretically incommensurate with Hermeneutic Constructivism and the actively languaging person that is the subject matter of focus.

\section{STEP 6 - VERIFY THE DESCRIPTIONS WITH THE PARTICIPANTS.}

Researchers from within the Colaizzi (1978) tradition are compelled to return the exhaustive statements to the participants in order to ensure that their perspective has been effectively captured. At face value this practice appears to be a decidedly good one. However research suggests that the philosophical contextualisation of meaning and the questionable ethical implications of such an approach is open to challenge. ${ }^{45}$ It is suggested here that when participants identify with aspects of a thematic representation, it should not be assumed to indicate that this representation is in fact representative, in the genuine sense, of that specific person. Instead such an identification could simply be a function of the level of generality or a zooming out from the deeper aspects of understanding that a thematic representation provides. Here, as in the earlier Google Earth analogy, the person is certainly able to identify with the representation, even though it is less representative of his or her self.

\footnotetext{
${ }^{44}$ The following chapter will emphasise that in terms of generalisation, Hermeneutic Constructivism is concerned with the way that a prejudice can be generalised to the overall function of that same person's greater system of prejudices.

${ }^{45}$ For a consideration of some of the issues of returning the transcription of interview data to participants see: Parker (2005) Qualitative psychology: Introducing radical research, as well as Poland (2003) 'Transcription quality' in Holstein and Gubrium, Inside interviewing: New lenses and new concerns.
} 
The Dialogical Experience Profile underpinned by the model of the Intersection of Understanding does indeed support the dialogical confirmation of understanding. That is, prior to directing the dialogue toward the elaboration of subsequent prejudice(s), the researcher clarifies the adequacy of the understanding that has been achieved of the participant's experience. Here the researcher projects his or her Mot Juste or the most perfect words for accenting the understanding thus far of the participant's experience. In so doing, the researcher seeks to establish a fusion of horizons between what is understood and the experience of the participant. Thus, in the fulfillment of the Dialogical Experience Profile, the requirement for post hoc verification of researcher's analysis is overcome through the dialogical movement that is epitomised by the model of an Intersection of Understanding. So much is this the case, that nothing further needs to be verified in excess of what has already been brought to understanding, and subsequently confirmed as adequate, by the participant.

The preceding discussion has emphasised that the level of abstraction with which Hermeneutic Constructivism is concerned does not lend itself to presentation in the same way as the thematic representations that pervade qualitative research. In light of this, a consideration of the way in which it is anticipated that a person's prejudices and their subsequent elaboration will be presented still stands in need of elaboration.

\section{The Hermeneutic Constructivist Presentation of an Inner Outlook}

At its core the development of the Hermeneutic Constructivist position is concerned with the presentation of human experience at a considerably high level of abstraction. It is anticipated that the Dialogical Experience Profile, along with the application of its subsidiary approaches, provides a vehicle for illuminating these fundamental ontological structures which condition the qualities of internal experience. If indeed the Dialogical Experience Profile is able to provide a representation of a person's prejudices, and their subsequent elaboration from authentic consciousness, then the profile itself represents the 'inner outlook' of the person. When considered in this way, the Dialogical Experience Profile may give the impression that it represents a neat and elegant presentation of the inner outlook. However some preliminary applications of the profile would suggest otherwise. In fact, the completed profile itself is covered with copious notes about the movement of laddering, or the elaboration of a prejudice. Although these notations are invaluable for the researcher's ongoing progress through the profile, they require elaboration in the presentation of such work to another audience. Importantly this elaboration is not a process of the researcher bringing meaning to the participant's outlook as though it is otherwise devoid of meaning. Instead it is recognition that the Dialogical Experience Profile is a difficult document to navigate without a textual elaboration. 
It is envisaged from the preliminary applications of the Dialogical Experience Profile, that the following structure provides a logical means of presenting the profile itself. The researcher begins by presenting the most superordinate prejudice(s) identified within a participant's system. This will provide an opportunity for the researcher to trace the influence of these structures upon the other prejudices that have been identified within the profile and even trace this prejudice back to the self-characterisation sketch itself. For each prejudice including the most superordinate structures identified - the researcher elaborates the context of the prejudice. Thus the Evident prejudice and Antithetic prejudice, along with any elaborative information garnered from the participant's authentic consciousness that has been substantiated through the intersection of understanding, is set down.

The researcher then seeks to represent the horizon of the prejudice, outlining the way that the prejudice itself brings an event to understanding for that person. As mentioned earlier, asking the participant to identify his or her present and ideal position along the spectrum between the Evident and Antithetic prejudice can be further illuminative of the prejudice itself. Furthermore, any discussion or more specifically any intersection of understanding about what it might take for a participant to move from the present to the ideal position - along the spectrum of the prejudice's context - should be incorporated into the discussion about that specific prejudice. This will heighten the overall understanding of the prejudice and therefore the representation of the participant that is presented. By following the structure outlined here, it is anticipated that a deeply nuanced and idiographic presentation of a person's inner outlook for qualitative research can be achieved.

In order to provide a theoretical exemplar of how this model of presenting the inner outlook might come to light, a return to the example of Jill Symons is useful. Let us assume that Jill situated herself presently at the Hates to talk about her son end of the spectrum and her ideal position at the Able to talk about her son position. In the process of describing what would be required to shift to the ideal position, Jill might suggest that she would need her parents to be more accepting of having a grandchild like her son; or that she would need to find a partner who had different priorities than the biological father of her son. These two examples highlight the way that a representation of this dialogue is clearly elaborative of a person's experience and provides further depth to the developing understanding of that person. On this basis, special consideration is given to the presentation of the prejudices that the researcher has selected as a vehicle for discussing what would be required for a participant to move from a present position to an ideal position. 
In the textual presentation of these elaborations, the researcher provides a representation of the dialogue of languaging that the participant engages in while moving toward his or her ideal position. Importantly this is not a representation of the dialogue that the researcher believes to have occurred. Instead it is a representation of the intersections of understanding that have been substantiated by the participant. It is the textual presentation of the researcher's Mot Juste, or the most perfect words for the understanding of the participant's inner outlook, that has been substantiated as sufficient by the participant in an intersection of understanding.

Despite the unapologetic focus upon the inner outlook of the individual person, as per the Similarity Corollary, the possibility of research making a statement about the extent to which two or more people can be considered similar is not dismissed. Recall that here the notion of similarity is considered in terms of a similarity with the way that people language their prejudices. As outlined previously, similarity at this level is not to be confused with a similarity in authentic consciousness as experience. That is, although two people may demonstrate a degree of similarity in their prejudice(s), each person's prejudice will indeed open up toward vastly different connections or elaborations in his or her authentic consciousness. Thus if claims to similarity are to be made, it is suggested that the researcher should always begin the presentation of such similarity with the statement 'It is the researcher's opinion that ...'. Here the researcher makes clear to the reader that this similarity is not explicit within the data, rather it is a view that has been imposed upon it.

\section{Chapter Summary}

Here a method that is commensurate with the fundamental theoretical principles of Hermeneutic Constructivism has been presented. The Dialogical Experience Profile and its subsidiary approaches provide a vehicle for discovering and presenting the structures that condition the qualities of internal experience and the comportment of being. The setting down of the prejudice(s) itself and its illumination through authentic consciousness, represents the inner outlook of the individual person for qualitative research that adopts a Hermeneutic Constructivist approach. Informed by the model of the Intersection of Understanding, the movement of the Dialogical Experience Profile itself and the inner outlook of the participant that is subsequently presented is not the product of the researcher's intuition or authority as a master interpreter. Instead it is a representation of a real person at a particularly high level of abstraction. It is a representation that has been substantiated as adequate by that person in the dialogical encounter itself. Given that the application of the Hermeneutic Constructivist approach is beyond the scope established for this thesis, the presentation of research outcomes that are informed by this approach remains speculative. 
Despite this, high hopes remain for its future application as well as for the possibilities that may arise from these theoretical developments more broadly. The productive possibilities that Hermeneutic Constructivism opens up is therefore the topic of the final and next chapter. 


\section{CHAPTER 9}

\section{DISCUSSION AND CONCLUSIONS}

Hermeneutic Constructivism has now been set down both theoretically and in application. This chapter will consider the way in which

Hermeneutic Constructivism can be considered to address the aims of the thesis and in so doing provide a redress to the previously identified challenges levelled at contemporary qualitative research and Personal Construct Psychology. This chapter will culminate in a discussion concerning the productive possibilities that the Hermeneutic Constructivist position opens up for future consideration.

\section{Looking back in order to redress the future.}

In order to begin a discussion concerning the way that Hermeneutic Constructivism can be seen to address the aims of the thesis, a review of how this journey began is useful. Hermeneutic Constructivism represents a theoretical response to a challenge levelled at two central areas of qualitative research: (i) the representational nature of qualitative research, and (ii) the criteria against which qualitative research is to be considered valuable. A considered sketch of these core challenges will now be outlined in conjunction with a discussion of how Hermeneutic Constructivism can be considered to be a suitable redress to these challenges.

\section{The Representational Nature of Qualitative Research.}

The challenge levelled at conventional approaches to qualitative research was couched in terms of a question:

Is it possible that qualitative research has contributed less than would seem attainable to the study of individuals because we as qualitative researchers have been working on the basis of a number of assumptions about people, which we have not examined very carefully, and have also adopted a number of assumptions about the nature of science which are less than adequate for the subject matter we have chosen?

The critique of contemporary approaches to qualitative research emphasised the problematic nature of several central assumptions that are necessitated by its practice. These assumptions form the foundation for challenging the claim that qualitative research can legitimately make to the representation of the experiences of real people. The core representational challenges 
levelled at qualitative research can be captured in terms of the following statements: (i) the acceptance of the naïve assumption that language simply designates things and therefore carries unambiguous meaning that is somehow transparent, (ii) the pervasive view that 'zooming out' to a broader or thematic representation is representative of the real people and real lives encountered in qualitative research, and (iii) that merely producing a good tale can somehow substitute for inadequately representing the real people encountered. Each of these statements provides entrée to a discussion concerning the way that Hermeneutic Constructivism can be seen to redress these representational challenges, as well as the productive possibilities this new approach opens up.

\section{Redress for an Assumed Transparency of Language and Meaning.}

The theoretical unpacking of Hermeneutic Constructivism has established that the prejudice is the condition of our internal qualities of experience and that human comportment itself is always toward understanding. Thus an exploration of a person's prejudices is indeed a look at that person's inner outlook. That is, the way that he or she understands the world, as well as the possibilities that are available to this person in understanding the world differently. In accepting that language is the medium of being human, then, our understanding processes are inextricable from language also. Therefore, the prejudice itself and those aspects of authentic consciousness that the prejudice provides access to are all inextricable from language.

It follows that if the internal processes of understanding occur in language, then these processes can be brought to understanding for another person in that very same language. To word this another way, the person of Hermeneutic Constructivism can be articulate about his or her Self because the very structures that condition this Self are inextricable from language. Thus, in addressing the naïve assumptions about language and meaning that inform contemporary approaches to qualitative research, Hermeneutic Constructivism rehabilitates the expressivist account of language. This is the notion that language is disclosing of the world, and that it is the condition of all understanding, to the extent that an expression cannot be understood independent of another expression. In so doing, Hermeneutic Constructivism reinstates the essential role of the dialogical encounter in coming to an understanding of another person.

It could be argued that contemporary approaches to qualitative research are also founded upon this same centrality of dialogue. However the requirement for post hoc analysis that involves the selection of aspects of the dialogue that the researcher believes to be significant as well as the subsequent application of meaning to these statements by the 
researcher, in the complete absence of any real-time dialogue with the participant, certainly debunks this view. On the other hand, informed by the theoretical tenets of Hermeneutic Constructivism, the Dialogical Experience Profile and its subsidiary approaches capitalise on the dialogical opportunity of the research encounter itself, and provide a vehicle for illuminating the structures that condition experience. Underpinning the movement of the Dialogical Experience Profile is the structure of the Mobius circle and the notion of an Intersection of Understanding.

One will recall that the structure of the Mobius circle represents the notion of interpretation in all understanding through the symbolism of two points approaching unity that never actually achieve this unity. Symbolically, as the points of intersection converge, the interpretation is more adequate and vice versa. Through an ongoing dialogue that follows the model set down in the Intersection of Understanding the researcher seeks an interpretation that effaces itself as an interpretation. That is, the interpretation accents those aspects of the participant's inner outlook so adequately that it is not seen as an interpretation at all, and instead is seen as the mot juste, the most perfect words with which to capture the thing that is to be understood. Here then the Intersection of Understanding achieves its complete elaboration as the ongoing search for the mot juste of the inner outlook of the participant, a movement that is always with the real person who is after all the subject matter of focus.

In describing the way in which the Dialogical Experience Profile is to be applied, it was suggested that the participant and the researcher move dialogically in an effort to achieve an Intersection of Understanding prior to moving to an elaboration of any subsequent prejudice. Therefore it is suggested that the researcher and the participant continue to move dialogically around one prejudice until the researcher achieves a genuine representation. That is, the researcher seeks the mot juste that represents his or her understanding, thus far, of the participant's prejudice. The participant is asked to provide feedback about the degree to which the mot juste of the researcher is commensurate with the participant's prejudice. It is therefore anticipated that the notion of an intersection of understanding that is implicit within the Dialogical Experience Profile, overcomes the naïve assumption about language and meaning that is common in conventional approaches to qualitative understanding. ${ }^{1}$ In fact,

\footnotetext{
${ }^{1}$ The capacity of conventional approaches to qualitative research to provide a genuine representation of the actuality of the real lives and real people encountered has been critiqued previously. An appropriate analogy that embodies these earlier challenges is the children's game 'Chinese Whispers'. Here one child whispers something to the child next to them and that child to the next and so on it goes until the message eventually returns to the initial child, by which time the message - adjusted slightly by each child - resembles something less representative of the original message. In the context of qualitative research, the naïve assumptions about language and meaning that have been traced through several key moments in the qualitative research process operate to shift the representation further away
} 
the legitimisation by the participant of the mot juste achieved by the researcher closes down the requirement for the generation of post facto meaning and representations. Both of which are necessitated in contemporary approaches to qualitative research.

As emphasised previously, the ontological primacy of the prejudice, as the essential condition of the comportment of being toward understanding, means that the illumination of these structures is in fact an illumination of the Self. The level of abstraction of the prejudice and the inherently idiosyncratic nature of authentic consciousness that each prejudice provides access to, means that carrying out any analysis after the dialogical opportunity itself has passed is a theoretical inconsistency. Instead prejudices and those aspects of their elaboration from authentic consciousness that have been substantiated as a Mot Juste of the participant's inner outlook are what is ultimately presented as a genuine representation of the participants. $^{2}$

\section{Redress for Thematic Generalisations as a Genuine Representation}

Irrespective of the language that anyone uses, a theme $i s$ a generalisation. It is a generalisation founded upon the degree that the researcher identifies a level of commonality between the statements made by participants, in the absence of the participant. More specifically, this is a commonality between the statements that the researcher has identified as significant and subsequently infused with meaning. Therefore, at the core of thematic representations, is the meaning that the researcher intuits or guesses from a participant's dialogue. In combination these intuited meanings become themes that supposedly represent the common threads of each person's experience. The necessity for a subscription to a naïve account of language that carries unambiguous meaning, and therefore an assumed homogeneity of what a statement means to each participant, is palpable. It has been emphasised previously that a representation founded ultimately on the prerogative of the researcher, while it may highlight aspects of an experience that a person can recognise as possible, is in fact not representative of the totality of experience of any one individual person. This notion is captured succinctly through the previously outlined analogy of thematic approaches with Google Earth.

from the actuality of the real person of focus. So much so that we begin to question who or what is exactly being represented by such approaches.

${ }^{2}$ On the topic of ethical issues arising from the representation of real people and real lives, Brinkman and Kvale (2004) believe "the phenomenological project of truthfully describing the world [to be] the most promising way to deal with ethical issues in qualitative research" (p. 175). From the perspective of Hermeneutic Constructivism there is not a higher order abstraction with which to uncover how a person goes about 'describing the world' than the prejudice. In light of this, Hermeneutic Constructivism provides an opportunity to represent ethically the inner outlook of real people encountered in qualitative research. 
The practices that are pervasive not only to thematic representations, but contemporary approaches to qualitative research more broadly, have been likened to a zooming out from the subject matter of focus. Here that subject matter is the inner outlook of the individual person. However, a thematic approach to understanding represents a movement away from this inner outlook of the participant to an outward inlook of the researcher. The view that a researcher could develop a outward inlook that is in fact a genuine representation necessitates a subscription to a naïve assumption about language and meaning. In fact, a qualitative researcher must accept that there is a transcendent position that somehow allows the researcher to get behind the words of a participant in order to know the connections, and meanings that these words make for that person, in the absence of that person. ${ }^{3}$ However, to use the words of Merleau-Ponty (1945/2003), "the system of experience is not arrayed before me as if I were God, it is lived" (p. 354). There is no 'God's eye view' from which to develop a legitimate outward inlook or representation of one person, let alone several people.

The founding principle of thematic analysis is a similarity between people. Hermeneutic Constructivism, by way of the Similarity Corollary, suggests that a similarity in the way that two people language the world is not to be confused with a commonality of meaning or experience. In fact, at the level of the prejudice - as representative of the Selfthe suggestion of a generalisation between people is likened to seeking similarity between apples and oranges. In order to achieve such a generalisation, one would need to zoom out to a common position such as fruit, a position that is certainly devoid of the unique and deeper qualities of each entity alone. Thus the focus of Hermeneutic Constructivism is to provide a focused consideration of the inner outlook of the person for qualitative research. This idiographic focus is more than merely another perspective from which to envision the human being, it is recognition of the nature of what being human is itself.

\section{Redress for Presentation of Participant Representation.}

Given that the Hermeneutic Constructivist position stands in need of future application, a speculative account was provided of how qualitative research informed by this

\footnotetext{
${ }^{3}$ In the words of Latour (2000), being ethical in qualitative research is letting the objects object to what is said about them and done to them. In line with this, a clear disconnect can be seen between the sentiments of Latour (2000) and the actuality of conventional practice. The insurmountable asymmetrical power and interpretive monopoly over the participants' representation in the research enterprise are examples of this disconnect. In contrast, Hermeneutic Constructivism seeks to illuminate a person's prejudices and those subsequent aspects of his or her authentic consciousness. In terms of Latour's (2000) sentiments, there is nothing to be presented in the final research outcome beyond a considered elaboration of the Dialogical Experience Profile and the presentation of those aspects that have been substantiated by the participant in the intersection of understanding itself - the fusion of horizons.
} 
position might be presented. The landmarks that pervade contemporary qualitative research presentations were offered as a backdrop for considering the differences that will be seen in the future presentation of Hermeneutic Constructivist outcomes. Central to the presentation of qualitative research outcomes informed by Hermeneutic Constructivism is the presentation of real people and real lives, and not simply proficient journalism and the production of a good or plausible read. As already outlined, one common tactic in the production of a good read is the presentation of a theme heading and subsequent verbatim quotes. This gives the impression that the theme is not only a natural progression from these quotes, but that the quotes are encompassing of the participants experience as a homogenous group.

In terms of Hermeneutic Constructivism, the Dialogical Experience Profile and its associated approaches provide a vehicle for representing a person's prejudices and their subsequent elaboration from authentic consciousness for the purpose of qualitative research. The application of the Dialogical Experience Profile does not require any post hoc analysis beyond the inner outlook that is represented and substantiated through the real-time dialogical encounter with the participant. Therefore, what is incumbent upon the researcher adopting this approach, is to bring the Dialogical Experience Profile to textual representation.

In remaining consistent with Hermeneutic Constructivism's conception of Person the Languager, it is important to recognise that each reader brings his or her own Self to the task of understanding. That is, a person always brings his or her system of prejudices and his or her subsequent authentic consciousness to every event of understanding, including the reading of a manuscript. Thus, ultimately, the reader will extract from a piece of work those aspects that resonate with him or her, and will make determinations of such things as the way that the participants represented can be considered to be similar. As outlined previously, the setting down of any similarity beyond a similarity in languaging is outside the theoretical purview of Hermeneutic Constructivism. However, if a reader envisages a degree of commonality within the representations presented that is deemed useful, Hermeneutic Constructivism has proved productive for this reader's purposes.

\section{Judgement Criteria}

Despite the aspirations held for the representational capacities of Hermeneutic Constructivism, the criteria or backdrop against which this representational capacity can be determined remains unconsidered. In order to situate Hermeneutic Constructivism with regard to a series of criteria, a brief sketch of the Criteria Debate is valuable. Concerned primarily with the notion of validity, the criteria debate is a consideration of the appropriateness or otherwise of criteria for making determinations concerning the legitimacy 
of qualitative research. Sparkes (2001) provided a useful framework by which to navigate what can only be considered a quagmire, consisting of three distinct streams of argument: (i) The letting go perspective suggests that the philosophical underpinnings of qualitative research do not lend themselves to judgement against criteria. Instead it is argued that one should allow the persuasive nature of the text to portray a tale that can be recognised by the reader. (ii) The parallel perspective accepts that the notion of validity is a worthy pursuit. However this pursuit warrants a series of new criteria that are specific to the task of qualitative research. (iii) The replication perspective argues that the traditional criteria for judging the legitimacy of scientific outcomes can and should equally be applied to the qualitative paradigm. In fact it is this perspective that will be used as a means of assessing the degree to which Hermeneutic Constructivism can make a claim to representing real people and real lives, and therefore make a claim to realism.

To paraphrase the words of Kelly (1955), 'The purpose of this thesis is primarily to expound Hermeneutic Constructivism, rather than defend it' (p. 392). Despite this, given that the compatibility of Hermeneutic Constructivism with the realist end of the spectrum has already been established, it follows that, the representations of the 'inner outlook' of real people produced are also tied to realism. What remains to be considered is the way that this inner outlook can be shown to embody the principles of validity, reliability and generalisability that are at the core of the replication perspective. Prior to a consideration of this, a brief look at why the replication perspective, and not the parallel perspective, was adopted as a backdrop is provided.

\section{The Replication Perspective}

It is unfair to anticipate that qualitative research can be considered in terms of the same statistical power reserved for the judgment of empirical research. However this acknowledgment does not dismiss the possibility that Hermeneutic Constructivism can or should be considered in terms of the principles embodied by these same criteria. Therefore the development of a series of parallel or supposedly different judgement criteria that would more adequately account for the different $r a w$ data that qualitative research concerns itself with would be unnecessary and replete with theoretical folly. Indeed, as has been shown, the notion of a parallel perspective and a different series of criteria ultimately succumb to the challenge of the Private Language argument first outlined by Wittgenstein. ${ }^{4}$ That is, one

\footnotetext{
${ }^{4}$ Wittgenstein's Private Language argument has previously been explored in Chapter Five. Briefly, however, Wittgenstein (1953/1968) in his Philosophical Investigations established that the development or invention of a private language is an impossibility without recourse to existing
} 
cannot begin to talk about the principles of parallel criteria or notions of trustworthiness or credibility etc. without recourse to the traditional criteria of validity, reliability and generalisability. Similarly Smith (1997) describes the notion of parallel criteria as being "parasitic" (p. 8) upon the core notions of the traditional criteria that the replicative perspective explicitly acknowledges.

In the same way that the notion of realism has been described in terms of a continuum between absolute realism and absolute idealism, the criteria with which judgments about the value of qualitative research are made can be considered along the spectrum between the validation of a universal truth and no criteria. On the one hand, an alignment with the replication perspective naturally discounts any subscription to a no criteria position. On the other hand, the possibility that a universal truth - therefore an absolute realism - can be achieved, let alone a series of criteria to ensure its achievement, represents a philosophical utopia. What remains for Hermeneutic Constructivism, then, is to be situated along this continuum in a manner that offers utility, while remaining theoretically logical.

The impetus for the development of the Hermeneutic Constructivist position was ultimately a perceived dissatisfaction with the capacities of contemporary approaches to qualitative research to represent the experiences of real people and the real lives encountered in research. Central to this dissatisfaction are considerations of validity. Thus, in response to this dissatisfaction, the Hermeneutic Constructivist position was established as a theoretical position for the conduct of a different depth of qualitative research that is capable of embodying the core principles of the replication perspective. The two most fundamental of these principles are: (i) the extent to which what was intended to be represented is indeed represented at the completion of the study, and (ii) the extent to which this representation is consistent, and therefore one that can bring about a parallel representation in the future. ${ }^{5}$ Commensurate with notions of validity and reliability respectively, a consideration of the immixture of these principles within the Hermeneutic Constructivist position is now provided in order to emphasise its compatibility with the replication perspective.

\footnotetext{
language use. He would argue that the development of terms such as 'trustworthiness' cannot begin to be understood without deferment to the notions or principles of validity.

${ }^{5}$ It is acknowledged here that the very nature of being human means that the bringing of an identical understanding in the future is not a likely or philosophically supported position. However, in the same way that a $90 \%$ Confidence Interval recognises that although not everything will be or is expected to be elaborated in the same way by that person in the future, there should be a degree of consistency.
} 


\section{Validity}

Convention holds that validity is a concern with the extent to which the focus of consideration is in fact what is being represented in the final product. In other words, it is the degree that what was intended to be measured is being measured by the methodological application of a theoretical position. In the context of Hermeneutic Constructivism, validity is therefore a determination of the extent to which the inner outlook of the individual person is represented in the final outcome.

As has been outlined previously, qualitative research traditionally adopts a series of tactics in order to give the impression that the principles of validity have been adhered to. Tactics common to contemporary approaches to qualitative research are: (i) the production of complete verbatim transcripts of interviews, (ii) the use of verbatim quotes as evidence for theme development, (iii) the use of member checking or confirmation of the themes by participants, and (iv) the use of co-researchers as instruments of confirmation. However it is suggested here that these tactics are merely a smoke-screen covering the implications of more pervasive theoretical assumptions. It has been suggested that the most central assumption is the subscription to a naïve or designative account of language. In such an account, language carries unambiguous and transparent meanings that the researcher is able to access as a master interpreter. Such an assumption has been shown previously to underpin the challenge that contemporary qualitative research is unable to produce representative or reflective accounts of the subject matter. The pervasive perpetuation of these theoretical assumptions represent what has previously been described as a refraction, or a bending away from the representation of real people and real lives.

In light of the challenge levelled at the capacity for contemporary approaches to qualitative research to represent real people, it is important to consider the way that Hermeneutic Constructivism itself can lay claim to representing these real people and real lives. However it is important to recall here the significant differences between the claim made by Hermeneutic Constructivism to the representation of a real and a claim to the representation of the real. This is recognition that what is experienced or understood by a person is indeed real for that person. From the perspective of Hermeneutic Constructivism it is the prejudice that conditions the comportment of being toward understanding and therefore the qualities of our internal experience. It is these same structures that allow each of us to have this real. Thus, given that the prejudice as well as those aspects of authentic consciousness that it provides access to are inextricable from language, the person of Hermeneutic Constructivism is able to be articulate about the way that he or she envisages the world and therefore what is indeed a real for that person. 
For Freeman, deMarrais, Preissle, Roulston and Pierre (2007), "there are no pure raw data, uncontaminated by human thought and action, and the significance of data depends on how material fits into the architecture of corroborating data" (p. 27). However, situating the prejudice as the condition of the qualities of our internal experience - a structure that provides entrée to the dialogue of languaging and is itself inextricable from language - challenges this view. As the essential precondition of the possibility of human understanding and therefore of the comportment of being, the prejudice, and its elaboration through authentic consciousness, represent raw data indeed. Moreover, given the depth of abstraction with which Hermeneutic Constructivism is concerned, the search for corroborative data as a measure of the data's significance has already been emphasised as being philosophically and theoretically incommensurate within a Hermeneutic Constructivist position more broadly.

In terms of validity and the degree with which the material under investigation is actually that which is being represented by the final product, Hermeneutic Constructivism is conceptually sound. It has been established that it is the inner outlook of the person that Hermeneutic Constructivism seeks to uncover. It follows that if the inner outlook of the person is conditioned by the prejudice and the dialogue of languaging that it provides access to, then illuminating these structures and presenting them as a final product of research is a valid representation of that person. Thus the application of the Dialogical Experience Profile and its subsidiary approaches provide a vehicle for representing an inner outlook that is substantiated by the participant. This would be a representation of not only the way that the participant envisages the world, but also the possibilities of envisioning the world differently for the purposes of qualitative research.

\section{Reliability}

Reliability is a consideration of the degree to which the results obtained are considered consistent over time or replicable (Golafshani, 2003; Hammersley, 1992; Joppe, 2000; Kirk \& Miller, 1986; Silverman, 2006). In other words, this is the extent to which the same instrument will yield similar findings that are independent of accidental circumstances alone. To couch this notion in terms of the Hermeneutic Constructivist approach, reliability concerns the extent to which the Dialogical Experience Profile illuminates prejudices with a degree of consistency with respect to time. Situating Person the Languager as the subject matter of concern may at first glance make reliability appear to be a difficult and philosophically incommensurate enterprise. That is, the subject matter of focus is the inner outlook of the person going about the business of being human, a business that by its very nature embodies change and does not lend itself to a state of stasis that would be seemingly required of reliability. A brief sketch of the way that conventional qualitative research deals 
with reliability will provide a touchstone for highlighting the developments gained by Hermeneutic Constructivism in terms of this notion.

It has been emphasised that agreement about what constitutes sound qualitative research and subsequently the way that this degree of soundness is determined remains fiercely contested within the literature. The notion of reliability is equally not exempt from this contest. Silverman, a prolific contributor to the critique and ongoing development of qualitative research, supports the use of reliability as an appropriate scientific standard for assessing the soundness of qualitative research. For instance, he (2006) outlines the approaches that are common to establishing reliability in a qualitative study: (i) the use of standardised methods for transcription of interview data, (ii) that the researcher performs the transcription, and (iii) multiple researcher analysis and comparison of such. However, while these procedures, most notably the use of multiple researcher analysis as a source of interrater consistency, are at best able to support a consistency in what has been made of the data (Silverman, 2006; Kirk \& Miller, 1986; Searle, 1999), these concerns are in fact outside the scope of reliability proper. Instead reliability is concerned with the degree to which the representation produced is consistent with future representations of the same person. Such concerns are with the extent to which two researchers identify the same aspects of a participant's dialogue as significant. Hermeneutic Constructivism provides grounds for a redress to this oversight, as well as an opportunity to situate the notion of reliability proper within future qualitative research.

Through the preceding theoretical unpacking of the Hermeneutic Constructivist position, one will recall that the Organisation Corollary recognises the way that each prejudice is integrally related, and that this relationship between prejudices has an implicit degree of consistency that operates to give the Self its overall sense of stability. This stability is determined by the extent to which a person shifts or evanescence from one prejudice toward another, in a way that is sufficiently stable to be more than mere happenstance alone. It was also emphasised that this stability is a function, ultimately, of the more superordinate structures of a person's system of prejudices and the way that these structures inform those prejudices that they subordinate. In order to emphasise the reliability that can be seen within this system, a look again at the historical nature of the prejudice is useful.

The prejudice has been outlined as that something that we have made of the world in past ventures of understanding and is that same thing that we can now project toward the world in order to illuminate it in understanding in the present. All understanding occurs in the dialogue of languaging that the prejudice not only provides entrée to but also sustains by way of those aspects of authentic consciousness that the prejudice provides access to. Given 
that the comportment of human being is always towards understanding better or differently, the dialogue of languaging is always directed toward the development of our prejudices in order to illuminate the world more adequately in the future. Thus the prejudice epitomises how time-past can be seen in and as essential to understanding in time-present. The person that Hermeneutic Constructivism has in mind always envisages the present from the perspective of what has been made of the past. Here, then, it follows that the higher order prejudices, which are more stable, are able to yield a greater influence upon the system. For example, given the integral nature of the prejudice system, a person who has faith in God as a higher order prejudice, and who therefore wishes to be a good Christian, will have developed subsequent prejudices from the perspective of this core prejudice. Thus each of the subsequent prejudices developed will be imbued with these to be a good Christian undertones. It is ultimately the influence of these superordinate prejudices upon the subordinate prejudices that gives these core structures consistency. This is so much the case that an encounter with evidence that challenged the existence of God, and therefore this core prejudice,would be vigorously languaged or perhaps ignored in an effort to ensure the ongoing stability of the greater prejudice system. Despite the stability in these higher order prejudices, it is important to appreciate that the more subordinate a prejudice is the less it is informed by this core prejudice and the more likely it is to be open to sweeping change. For example, the prejudice chocolate milkshakes are best can change rapidly in the presence of a vanilla malt milkshake, without there being any effect on the good Christian prejudice (unless of course it is a Sunday). Importantly, the more core a prejudice is the more it is able to influence a person's system of prejudices and therefore the more stable it is.

The Dialogical Experience Profile and subsidiary approaches, most notably the selfcharacterisation sketch, provides a vehicle for illuminating a person's Evident prejudice or the prejudice that readily comes to hand for consideration. It is envisaged that focusing the selfcharacterisation sketch at the level of experience with and of phenomena suggests that the Evident prejudice(s) illuminated will be at a level that is more superordinate than the level of a preference for a flavour of milk. It has also been suggested previously that it is possible that the Evident prejudice(s) illuminated are already of a significantly high order within a person's system of prejudices. However, it is important to appreciate that while not all the Evident prejudices are at this level of abstraction, they are certainly not the most subordinate structures within the prejudice system. In light of the possibility that a person of Hermeneutic Constructivism can engage with and elaborate his or her higher order structures, the use of laddering has been employed. Moreover, the outcomes of laddering are reinstated as an exemplar of how Hermeneutic Constructivism can be considered consistent with the notion of reliability. 
Laddering, in its turn, allows the researcher to uncover higher order structures within a participant's prejudice system in order to illuminate the more influential aspects of that system and therefore provide a deeper inner outlook for qualitative research. In relation to the notion of reliability, then, it is the stability of these higher order structures and their subsequent consistency or persistence within that person's prejudice system that allows a claim to reliability to be made. Thus the integral nature of the prejudice system, as well as the the inherent stability among higher order prejudices, suggests that the application of the theoretical principles of Hermeneutic Constructivism is able to provide a reliable inner outlook. This would be a representation that will yield a consistency amongst the higher order structures extracted.

\section{Generalisability}

Finally, generalisability embodies a consideration of the degree to which the findings of a study can not only be considered applicable to, or representative of, all entities within the study, but also those beyond the study itself. In terms of Hermeneutic Constructivism, generalisability would be the extent that a prejudice can be generalised from one person to another person. This position has been emphasised as being theoretically incompatible with the theoretical tenets of Hermeneutic Constructivism more broadly. Nonetheless, despite this contradiction, the notion of generalisability as a backdrop for making determinations of a piece of research's soundness is considered valuable when conceptualised differently. In order to undertake a consideration of the way that Hermeneutic Constructivism envisages the notion of generlisability, it is first necessary to provide a sketch of how it is typically considered in qualitative research.

It has been emphasised that contemporary approaches to qualitative research involve the researcher's subscription to a series of theoretical assumptions that necessitate a naïve or designative view of language. In terms of generalisation, a researcher coming from this tradition, and therefore steeped in these assumptions, would first make meaning from the participant's words that he or she deemed significant. Furthermore, this same researcher would seek a degree of commonality with other individuals on the basis of these fabricated meanings. Despite the dubious nature of this consideration of commonality, there is a body of scholars who suggest that qualitative research should not be satisfied with a focus upon the particular. Instead these scholars argue that qualitative research can and should produce generalisable explanations with wider resonance. ${ }^{6}$ For example, Morse (1999b), a leading

\footnotetext{
${ }^{6}$ There is a body of work that supports the idea that that it is possible to generalise from qualitative studies (cf. Mason, 1996; Yardley, 2008). There are also authors who believe it possible to generalise
} 
figure in the field of qualitative research, provides one iteration of this argument for generalisability in qualitative research. She states, "If qualitative research is considered not generalizable, then it is of little use, insignificant, and hardly worth doing" (p. 5).

Envisaging generalisation differently, however, Hermeneutic Constructivism is concerned not with the way that a prejudice can be generalised to another person, but instead with the way that a prejudice can be generalised to a person's greater system of prejudices. Thus what is of central importance to Hermeneutic Constructivism is to highlight the way that subordinate prejudices are subsumed by higher order prejudices. Here parallels can be drawn with Noaparast's (1995) notion of construct coherence. ${ }^{7}$ In fact, to paraphrase Noaparast (1995), Hermeneutic Constructivism takes the view that the extent to which a person's prejudice can be traced back to and through that same person's greater system of prejudices or 'best theory', determines whether it can be considered to be generalisable to that person's theory.

This notion is also captured by Yin's (2009) notion of 'analytic generalisation'. Here Yin (2009) suggests that the findings that arise from a series of case studies should not be used as a vehicle for identifying generalisations to a population of people, but rather to support a theoretical position. To make this clear, he draws an analogy between analytic generalisation and the laboratory investigator who selects a number of experiments in order to support a particular theory. Yin (2009) suggests that "under these circumstances, the mode of generalization is analytic generalization, in which a previously developed theory is used as a template with which to compare the empirical results of the case study" (p. 38). In terms of Hermeneutic Constructivism, generalisation is a consideration of the degree to which a person's prejudice, as an experiment, can be seen to fit within or support that same person's greater theory.

As mentioned previously, it is possible that the movement of laddering different Evident prejudice(s) may arrive at analogous, if not the same, superordinate prejudice(s). It is here that laddering is able to emphasise the influence that a superordinate prejudice or its analogue has with regard to those prejudices that are subordinate to it. In other words, laddering is able to uncover the degree to which a superordinate prejudice, or way of envisaging the world, can be generalised to the wider system of prejudices. Thus a prejudice

from qualitative studies that have taken a case study approach (cf. Flyvbjerg, 2004; Kennedy, 1979; Stake, 1994).

${ }^{7}$ Noaparast (1995) discloses what he means by construct coherence in terms of the coherence of a single construct with a person's greater construct system. This is not to be confused as a coherence with reality per se. 
that can be traced through more of a person's system of prejudices can be considered to have a greater degree of generalisability. This entails that such a prejudice is indeed representative of that person's inner outlook.

Conceptualised in this way, generalisability provides Hermeneutic Constructivism with a powerful description of the influence of a person's core prejudice(s) on the broader aspects of his or her prejudice system. Nonetheless it is important to recognise that the search for generalisation, and therefore the influence of a higher order prejudice, is not the principal consideration of the application of the Hermeneutic Constructivism in qualitative research. Instead generalisability provides a means of making determinations of the soundness of a study that is informed by the tenets of Hermeneutic Constructivism. In addition it provides an opportunity to further evaluate the validity, and to a lesser extent the reliability, of the prejudice(s) illuminated. Worded another way, a prejudice is valid to the extent that it can be shown to be influenced by a higher order prejudice(s). In addition, a representation of the person's inner outlook is reliable, albeit not with respect to time, if analogous higher order prejudice(s) are illuminated in the movement of laddering.

A final remark about making generalisations from one person to another is useful. Hermeneutic Constructivism represents a position whereby comparison between individual people at the level of the prejudice is theoretically inconsistent. However the theoretical unpacking of Person the Languager is in fact reflexive to the notion that a reader of research informed by this position may decide otherwise. That is, no researcher can be certain from the outset whether or for what purpose a piece of research may be of use to another person. As suggested previously, it is always possible that a reader may suggest that the representation of a participant is sufficiently similar to that of another person beyond the study. So much so that the reader may be able to apply the understandings from that representation to his or her own situation. Kennedy (1979), in her work titled 'Generalizing from single case studies', shares this view. She suggests that clinical and legal cases can be suitable examples where parallel generalisations are made. Here a person applies an understanding from one situation to that of another situation that is considered to have parallel features. ${ }^{8}$ This situation is exemplified by both the clinician and the judge who make determinations about the degree that a representation accents aspects of a situation that are

\footnotetext{
${ }^{8}$ This is in line with Gadamer's (1960/2003) notion of application where one takes an understanding from one situation and applies it or brings it to life anew in another. Gadamer envisioned the Judge and the Religious Minister as examples of such application. See Truth and Method (1960/2003), pp. 304-311.
} 
considered to be compatible with the situation at hand. ${ }^{9}$ In terms of Hermeneutic Constructivism, then, an inner outlook that is produced could be envisaged by a reader as capable of informing an understanding of another person.

\section{Implications and Productive Possibilities of Hermeneutic Constructivism}

As the light draws dim upon the path taken to arrive at the Hermeneutic Constructivist position, a series of further paths await provocatively to be followed. This discussion will outline some of the implications, as well as the productive possibilities that the Hermeneutic Constructivist position offers, illuminating differently several familiar and yet convoluted paths. These include the implications of Hermeneutic Constructivism upon: Personal Construct Psychology, notions of the Self, and the future of qualitative research practice.

\section{Personal Construct Psychology}

To paraphrase Kelly (1955) once again, Personal Construct Psychology can be read between many of the lines of Hermeneutic Constructivism. Despite this, it is suggested that the Hermeneutic Constructivist position is capable of weighing into two heavily contested, and persistent debates within the field of Personal Construct Psychology itself. ${ }^{10}$ These include: (i) the Personal Constructivism vs. Social Constructionism debate, and (ii) the constructivist realism debate. In light of a consideration of the contribution of Hermeneutic Constructivism to this latter debate within the body of the thesis, a sketch of how Hermeneutic Constructivism can be considered to contribute productively to the personal and social debate will now be outlined.

\section{Personal Constructivism vs. Social Constructionism}

Within Personal Construct Psychology literature, a developing body of work can be seen which concerns the degree of compatibility between Social Constructionism and Personal Constructivism's respective takes on being human. ${ }^{11}$ Although some scholars argue that these positions can be viewed as already compatible by way of Kelly's original work (cf.

\footnotetext{
${ }^{9}$ The work of Freud (1963) provides a further example where in-depth single clinical cases have provided a wealth of information that remain a source for generalisation in contemporary research. ${ }^{10}$ The Adams-Webber (1990) / Warren $(1990,1991)$ debate, although perhaps the most vigorous contested explorations of the synergies between Personal Construct Psychology and Cognitive Psychology, it is not seen as being contributed to by way of this thesis and is therefore simply acknowledged and not delved into any further.

${ }^{11}$ Gergen (1985) prefers the use of the term constructionism in considerations of the social as a symbol of the lineage to the work of Berger and Luckmann (1966). Chiari and Nuzzo (1996a, 1996b) also support the use of the terms constructionism when referring to social elements.
} 
Butt, 1996, 1997a, 1998, 1999, 2000, 2001; Chiari \& Nuzzo (2003a); Fry, Butt \& Bell (2003); Kalekin-Fishman \& Walker, 1996; Davidson \& Reser, 1996; Raskin \& Bridges, 2004; Scheer, 2000; Warren, 1998, 2000, 2004), there are others who consider such claim to compatibility to represent a philosophical indictment (cf. Gergen, McNamee \& Barrett, 2001; McNamee \& Gergen, 1992; McNamee, 2004). ${ }^{12}$

Warren (1998) suggests that "Personal Construct Psychology was developed as a psychology of the individual, an idiographic psychology" (p. 72). In a similar way Stojnov and Butt (2002) suggest that Kelly’s (1955) theory “has been developed mainly as a mentalistic psychology, at the expense of an interpersonal approach to the person" (p.99). Despite this, Warren (1998) is sympathetic to a view of Kelly's work as being compatible with the more social aspects of being, at least in embryo. Indeed, Kelly (1955) was not ignorant of the socially situated nature of the person, and the amplification of his Fundamental Postulate, by way of the Sociality Corollary and Commonality Corollary, is testament to this. However, prior to returning to the social, personal debate, it must be reiterated that this thesis has suggested that the way Kelly (1955) unpacked this socially situated person is open to challenge on the basis of the way that he dealt poorly with language.

Encompassing the broader debate, Warren (1998) suggests that "social constructionism is less conciliatory toward personal construct psychology than the latter is to the concerns of the social constructionists" (p. 172). Proponents of Social Constructivism are concerned with the way that people engage in the activity of being human together, emphasising the relational nature of meaning making and understanding in the world. This is so much the case that McNamee (2004), a staunch adherent to the Social Constructivism movement, captures the stance towards Personal Construct Psychology that is common amongst scholars subscribing to the tenets of Social Constructivism. She suggests that "the most interesting aspect of Kelly's work...is how he eventually pulled himself from inside the head of the person into the social arena" (p. 38). Although Kelly (1955) acknowledged the principle of a socially embedded person, the sophisticated detail of this socially situated person is lacking in his work. In other words, a consideration of the medium through which

\footnotetext{
${ }^{12}$ Several authors within the arena of Personal Construct Psychology believe the Sociality Corollary is already the fundamental bridge between the previously incommensurate personal and social realms (cf. Warren, 1985; 1998; Walker, 1990; Butt, 1996). On the other hand, although Raskin and Bridges (2004) highlight some important connections between these two paradigms, Raskin (2011) suggests that there are also some notable differences. While other authors (cf. Duck, 1994; Harre \& Gillett, 1994; Mascolo, 1994) recognise the value of Kelly's approach, they also believe that what is required is a deliberate consideration of the way in which Personal Construct Psychology can be considered commensurate with some of the more Social Constructivist and interpersonal dimensions of being human.
} 
Kelly is able to pull Personal Construct Psychology from the head of the person and into the social domain is lacking. It is here that Hermeneutic Constructivism is able to provide a possible redress for the conundrum envisaged by McNamee.

Hermeneutic Constructivism has situated language as the essential medium of being human. Language is not only the medium of what goes on between people, but also the medium of what goes on within a person. That is, the structures that support the possibility of having qualia and the comportment of being toward understanding are inextricable from language. Therefore the person envisaged can be articulate about the inner processes of understanding. Hermeneutic Constructivism therefore provides a theoretical consideration of the way that a person develops lawfully a series of highly nuanced understandings of the world that can be articulated to one's self and to other people. In so doing it provides a way for the personal and social aspects of being to go on together.

Another noteworthy debate concerning issues of the degree to which Personal Construct Psychology is commensurate with Social Constructivism - a debate that Hermeneutic Constructivism might also inform a theoretical pathway through - is the Mancuso/Burkitt/Wortham debate. Mancuso (1996) proposed an amalgamation between the three traditions of Personal Construct Psychology, Narrative Psychology and Social Constructionism. Although Burkitt (1996) initially commended Mancuso for raising awareness of the possibility that there is much to be gained by Personal Construct Psychology taking more seriously the theoretical principles of Social Constructionism, he goes one to highlight a series of perceived shortfalls in Mancuso's position.

According to Burkitt (1996), the major challenge is the lack of attention that Mancuso gave to the unresolved tensions in the ontological foundations of these differing perspectives. That is, Burkitt (1996) argued that what remains to be achieved by Mancuso (1996) is to theorise "how individual constructs can appear within the broader framework of social constructions; in other words, how shared, socially produced text can be adapted and changed by social individuals in their practices" (p. 72). This provided the foundation for Burkitt (1996) to accuse Mancuso (1996) of solipsism through his reliance upon individually constructed meanings, and his subsequent neglect of a consideration of the social context of interaction and the actions of such engagement. Burkitt (1996) goes on to suggest that as a result of this solipsism, the only way that the person envisaged by Mancuso (1996) can come to an understanding of another person, is in terms of that "individual's thinking about the thinking of others" (p. 74). The argument established here by Burkitt (1996) is reminiscent of Wittgenstein's Private Language argument, where the person Mancuso (1996) envisages 
develops a series of private stories from which to construe the mental states of others. These stories, however, are beyond validation outside of that person's own private and closed narratives, thereby resembling a form of self-fulfilling prophecy. Thus, reminiscent of the languageless and pattern scanning nature of the constructs that Kelly (1955) emphasised, the ideological individualism that Mancuso (1996) envisages is presented as being removed from any shared position with which to claim a realism. These arguments lead Burkitt (1996) to finally assert that Mancuso's (1996) attempts at integration are not theoretically or ontologically coherent without further philosophical groundwork.

Drawing on a similar perspective to Burkitt (1996), Wortham (1996) also compliments Mancuso (1996) for lifting the debate beyond issues of what is real. However he suggests that Mancuso (1996) appears to be less adept with the theoretical tenets of Social Constructionism and consequently has not effectively taken into account the relational context of being human. As he sees it, Mancuso's (1996) commitment to Kelly's (1955) personal constructs, as mental or cognitive schemas with which one scans the world for meaning, does not sit comfortably with theoretical approaches that take relational and mediational notions of being as primary in the way that Social Constructivism does. Hence, although Wortham (1996) acknowledges Mancuso (1996) for identifying some points of intersection between Personal Construct Psychology and Social Constructionism, he believes that Mancuso's (1996) position is ultimately incompatible with Social Constructionism.

In theoretically situating the structures of ontological primacy in the medium of language, the Hermeneutic Constructivist position thus provides a redress that is consistent with a social constructivist position. In other words, the inextricability of the processes of understanding from language means that the person envisaged by Hermeneutic Constructivism is able to be articulate about these processes with other people, and to have future iterations of these processes developed as a function of that mediation. In so doing, Hermeneutic Constructivism provides a theoretical position that overcomes a subscription to a Private Language that is necessitated by Mancuso's (1996) adherence to Kelly's (1955) scanning model approach. To paraphrase the words of Harre and Gillett (1994) here, Hermeneutic Constructivism can be regarded as a theoretical position that has genuinely taken seriously both the tenets of Personal Construct Psychology and Social Constructionism. Given that any theoretical development should always be in the direction of seeking to better understand the subject matter of concern, it is anticipated that Hermeneutic Constructivism has provided an opportunity to further a consideration of the personal as well as the social aspects that are inherent in being human. 


\section{Notions of Self}

Hermeneutic Constructivism has been emphasised as a theoretical position that embodies the inextricability of language from the social and personal dimensions of being human. In other words, Hermeneutic Constructivism provides a consideration of what goes on between people as well as what goes on within the person. It is anticipated, therefore, that this position may illuminate different notions of Self. Although the study of the Self has a long and convoluted history, a review of this history is considered to be of little value beyond establishing a definition that stands as a foundation for elaboration. ${ }^{13}$

A useful definition provided by Baumeister (1998) suggests that notions of Self should be considered as "the capacity of the human organism to be conscious of itself as a distinguishing feature" (p. 683). Baumeister (1998) goes on to suggest that the Self consists of all that a person knows about him or her self. That is, one's likes, dislikes, beliefs, values, personality traits, emotions etc. Thus the Self represents the essential being of a person that ultimately distinguishes him or her from other people. In terms of Personal Construct Psychology, Kelly (1955) suggests that "the self is, when considered in the appropriate context, a proper concept or construct" (p. 131). So much so that for Kelly (1955) a glimpse at the constructs through which a person brings the world to meaning is a look at the Self. ${ }^{14}$

Ignoring for the moment the arguments levelled at Personal Construct Psychology by this thesis, it is the ability of a person to share with another person his or her construct system, and therefore notions of the Self, that makes the application of Personal Construct Psychology most valuable (Cox \& Lyddon, 1997). That is, the notion that the Self can come to language or the Self as Narrative, represents an attractive conviction for constructivist practitioners to hold. ${ }^{15}$ On this Mair (1989) takes the view that Kelly's (1955) work should be considered in terms of a story-telling psychology. He goes on to suggest that the essence of Personal Construct Psychology is to bring to meaning the Self of other people through this story telling. ${ }^{16}$ In order for Mair's (1989) position to hold he must assume a correspondence, at

\footnotetext{
${ }^{13}$ For a thorough consideration of the theoretical evolution of considerations of the Self, the following text is worthwhile. Taylor (2006) Sources of the Self: The making of the modern identity.

${ }^{14}$ While considering the links between notions of the Self and Personal Construct Psychology Bannister (1983) remained consistent with Kelly's notion of the Self as being inextricably linked to a person's greater construct system.

${ }^{15}$ It is in fact this conviction of Personal Construct Psychology that propels the work of Butt, Burr and Bell (1997) as well as Fry, Butt, Bell (2003). To paraphrase Fry, Butt and Bell (2003) "people certainly have a keen sense of self that pervades their interactions" (pp. 71, 76).

${ }^{16}$ Synergies can be seen between this notion of Self in story telling and Herman's model of the Dialogical Self. According to Hermans (2003) the dialogical self is "on the interface between James and Bakhtin" (p.90). For Hermans, Kempen \& Van Loon (1992) the dialogical self represents a conceptualisation of the Self as "a dynamic multiplicity of relatively autonomous $I$ positions in an
} 
some level, between the construct and language within Kelly's (1955) work. However, the way that Kelly (1955) situated the construct beyond the reach of language is in fact incommensurate with the position elaborated by Mair (1989). In order for a narrative or story telling elaboration of the Self to be sustainable, the medium of the Self-I relationship would need to be the same medium as the Self-other relationship. In other words, the way that a person's constructs or Self is shared with another person, cannot be different from the way that this person has a notion of Self for him or her self. Thus in order that the Self as a construct can be brought to language for another person in terms of Mair's (1989) story telling, means that a person must be able to bring his or her construct or the Self to language. This position is clearly out of step with Kelly's (1955) conceptualisation of the construct as having its being beyond the reach of language.

In a discussion titled Problems of the Self, Warren (1998) outlines the historical milieu of the notion of the Self and points toward the work of Cox and Lyddon (1997) as providing perhaps the most thoroughgoing consideration of the notion of the Self within the constructivist paradigm. These authors identify that the postmodern turn within the sociobehavioural sciences has challenged modernist, essentialised notions of the Self. ${ }^{17}$ In so doing, these authors believe several constructivist positions on the topic of the Self have been opened up as possibilities for consideration. Cox and Lyddon (1997), for example, identify five distinct positions ${ }^{18}$ from which the constructivist paradigm more broadly can consider notions of the Self. ${ }^{19}$ Considering the notions of Self that have been proposed by these

imaginal landscape" (p. 28). More specifically this conceptualisation holds that the $I$ has the capacity to fluctuate between a series of different positions. This fluctuation can occur because each $I$ position is endowed with a voice that allows a dialogical relationship between each of these different $I$ positions to ensue. Much like the characters in a story, each $I$ has a story to tell from its own perspective and contributes to a complex narratively structured Self. Thus the Self of Hermans, Kempen \& Van Loon (1992) and Hermans (1999, 2002, 2002a, 2003) is decentralised, in that it has no single, central, or even core, Self position. Instead, his Self is polyphonic, having a multiplicity of $I$ positions that all relate to each other. Despite the disconnect between the Self of Hermeneutic Constructivism and Hermans' Self, the shared centrality of dialogue - although not fully fleshed out within Hermans work - opens up the possibility of a theoretical synergy between these two positions in the future.

${ }^{17}$ According to Cox and Lyddon (1997), the postmodernist turn within the socio-behavioural sciences provides a "new worldview of relativity and relatedness, of process and context, of ambiguity and paradox. In contrast to modernism, the postmodern view underscores the way in which realities are personally and socially constructed" (p. 203). Therefore assumptions about truth within modernist thought are now open to "reawakened scrutiny" (p. 203) and the possibility of multiple realities. These conceptions are at the heart of the dynamic consideration of the universe that underpins postmodernism.

${ }^{18}$ For a complete review of the way in which Cox and Lyddon (1997) elaborate these five positions, see 'Constructivist conceptions of self: a discussion of emerging identity constructs'.

${ }^{19}$ These five positions include: (i) Self as Self-Theory which relates to the notion that if a person constructs experience, then that person also builds his or her Self. Here the authors focus on the work of Berzonsky (1990) who supports a re-examination of the philosophical assumptions that support scientific theory, and a subsequent re-think of the parameters of reality; (ii) Self as Evolving Process which follows the principles of evolution in that the Self is not a static entity but is rather ongoing in 
authors, Warren (1998) suggests that the discussion converges at a conundrum regarding the degree of individual agency that is embodied by each of the varying approaches. In particular Warren (1998) suggests that many contemporary approaches to the Self discredit the centrality of the subject. In contrast to this, Warren (1998) argues for the centrality of individual agency, and identifies four strategies for mounting this argument: (i) recount the attempts to catalogue a substantive account of the self; (ii) appeal to common experiences lived experience and emotional experiences - that have been devalued by the de-centering of the subject; (iii) develop a theory of self confluent with the "reasonable claims" of social constructivist position; and (iv) identify the shortcoming of a de-centering of the self (pp. 96, 97). Given that it is the final two positions that this thesis considers to hold most promise, a consideration begins with a discussion of the fourth position - identify the shortcomings of de-centering of self.

A complete deferral to the Social Constructivist position appears to give up on the notion of human agency, and ignores the unique potentialities of human experience. ${ }^{20}$ In fact, social constructivist positions have been criticised for providing a merely passive account of the human subject (cf. Colapietro, 1990; Raskin, 2002; Rychlak, 1990). Such passive, and inherently unreflexive accounts of the person, ultimately return to arguments concerning the notions of realism and idealism, notions that have already been described as devoid of closure. In light of this, it is toward the third and perhaps more ambitious strategy identified by Warren (1998) that attention is now drawn. In an effort to reestablish individual agency within notions of Self, Warren (1998) proposes to develop a theoretical position commensurate with the more reasonable claims of social constructivism.

Frank (1989), whose work can be seen as influencing Warren's (1989), suggests that despite the inherent problems he foresees in a hermeneutics of self-understanding, he cannot envisage the possibility of an alternate non-understanding Self. As a consequence, Frank (1989) believes that a "Hermeneutics of individuality of a sort in which the individual moment is not an opponent, but rather a moment of the structure" (p. 358), might allow a

development and refinement. Here the authors focus upon the work of Guidano $(1987,1991,1995)$ as well as Bugental (1980) and Bugental and Bugental (1984); (iii) Self as Transcendent. This embodies the influence of Western mysticism upon scientific thought and captures the evanescent notion of Self. For a thorough review of the core components of this discussion see Hunt (2003) Live in Spirit:

Precursor and Dilemmas of a Secular Western Mysticism. The final two considerations of the Self that Cox and Lyddon (1997) elaborate are: (iv) Self as Narrative; and (v) Self as Social/Economical and Political Construction.

${ }^{20}$ Proctor (2009) suggests that in the personal versus social debate what "we need is to strike the right balance. Too much towards the personal gives too individualist a position. Too much towards the social results in a loss of the lived realities of the actual people we are attempting to understand" ( $p$. $35)$. 
more positive notion of the Self to emerge. Hence, situating the essential structures of the human comportment toward understanding as inextricable from language, Hermeneutic Constructivism could be considered to encompass aspects of Frank's Hermeneutics of individuality, and may in fact open up towards a positive notion of the Self. Moreover, Hermeneutic Constructivism provides a redress to the de-centering of the individual and personal agency within notions of the Self that was alluded to by Warren (1998), instead representing a theoretical position that is commensurate with the more reasonable claims of Social Constructionism. By situating language as the central and common denominator in conceptualisations of the Self, from both the social and personal perspectives, Hermeneutic Constructivism therefore provides an opportunity for social and personal accounts of Self to begin to play on the same field, and for further dialogue to ensue. ${ }^{21}$

\section{The Epistemic Interview}

Brinkmann (2007) posed the following question of qualitative interviews: 'Could interviews be epistemic?' In other words, is it possible to achieve knowledge (episteme) over and above merely opinion $(d o x a)$ ? Concerned that the opportunity to uncover episteme is reduced to what happens after the interview, and not what goes on during the dialogical opportunity itself, Brinkmann (2007) draws attention to the way that the interview is carried out. He suggests that in the typical doxastic or qualitative interview, participants are asked to respond to questions concerning concrete episodes and experiences over and above any consideration of more abstract reflections. Echoing Denzin's (2001) call to move beyond qualitative research as story telling, and therefore mere doxa, Brinkmann (2007) defines knowledge as that which can be validated and/or justified within a person's practical reality. He therefore suggests epistemic interview techniques as a way forward for qualitative research.

Reflecting upon previous work that can be seen to have adopted an epistemic interviewing approach, Brinkmann (2007) identifies key aspects for such an approach. He believes, for instance, that an interviewer should press the interviewee regarding any contradictions in his or her elaboration or definition of experiences. The interviewer should then seek to determine the centrality of this definition by assessing the interviewee's

\footnotetext{
${ }^{21}$ One consideration of Self that Heremeutic Construtivistm might contribute productively to is that proposed by Angus, Levitt and Hardke (1999). These authors, in the words of Neimeyer and Levitt (2000), suggest that the Self is developed through "an endless series of micronarratives of daily events, eventually abstracting these into an overarching macronarrative of our lives that winnow out those relatively stable features... upon which we build a sense of identity" (p. 402). Some palpable synergies can be seen here between the work of these authors and Hermeneutic Constructivism.
} 
unwillingness to give it up. Here some clear synergies can be recognised between these strategies identified by Brinkmann (2007) and Hermeneutic Constructivism in application.

The prejudice as the essential condition of internal experience is a high order abstraction indeed. Thus a look at a person's prejudices is more than a look at how he or she defines an experience, it is instead a look at the structures that condition the possibility of experience at all. Moreover the prejudice has been established as existing within a greater system of prejudices that consists of integral relationships between each prejudice, where superordinate prejudices represent the greatest degree of stability or consistency within a person's Self. Thus illuminating these higher order prejudices by way of the Dialogical Experience Profile and its subsidiary approaches, is indeed consistent with illuminating those aspects of a person's understanding that he or she is unwilling to give up. Consequently, representing a person at the level of the prejudice is more than simply a doxastic representation developed by the researcher.

At the centre of Brinkmann's (2007) concern for the production of episteme is a desire for knowledge that better serves the community in terms of political, ethical and other normative issues. Central to his concern is a belief that our current understanding of such matters is too easily reduced to a psychological understanding in the form of experience and opinion. ${ }^{22}$ Although clearly the central focus of Hermeneutic Constructivism does not sit comfortably within the broader directions Brinkmann (2007) has in mind for his epistemic interview, there are some noteworthy synergies. There can be little doubt that the prejudice with which Hermeneutic Constructivism concerns itself is an abstraction of a sufficiently high level to be considered episteme in the way Brinkmann (2007) envisages it. Furthermore it has been established previously that the prejudice embodies a person's historicity as that something that has been made of being within the world. It represents the knowledge or the episteme that a person has thus far. Moreover the Dialogical Experience Profile shows some significant parallels with the broader parameters of the epistemic interview and provides a productive opportunity to pursue further qualitative episteme in the future.

\footnotetext{
${ }^{22}$ Brinkmann (2007) cites the work of Sennett (1977) who believes that social science should serve the political community by engaging a community in ethical, political and other normative issues. He goes on to argue that qualitative research would not simply bring understanding of the intimate aspects of being human, but would also bring aspects of political relations and other matters of communal value. Sennet (1977) goes on to emphasise his concern for a society that is reduced simply to issues of psychologism that invades the public space and destroys public life.
} 


\section{Conclusion}

Hermeneutic Constructivism has been proposed as a thoroughgoing theoretical response to the representational challenges that undermine contemporary practices of qualitative research. It has been shown that contemporary approaches to qualitative research are beholden to a naïve assumption that language carries transparent and unambiguous meaning. This challenge was identified as having profound implications for qualitative research. The challenges converge ultimately at the following question.

Is it possible that qualitative research has contributed less than would seem attainable to the study of individuals because we as qualitative researchers have been working on the basis of a number of assumptions about people, which we have not examined very carefully, and have also adopted a number of assumptions about the nature of science which are less than adequate for the subject matter we have chosen?

Realised as a response to these provocative questions, Hermeneutic Constructivism is a theoretical consideration of the way that a person goes about the business of being human in and through language. It offers a foundation for a qualitative research that has as its focus the elaboration of a person's innermost experiences or qualia. Reaching toward a focused consideration of the individual person's 'inner outlook' at a considerably high level of abstraction - the prejudice - has been shown, in theoretical terms, to be a most productive and reasonable avenue to pursue. As the essential condition of our innermost experiences, or qualia, a consideration of the person at the level of the prejudice not only provides a redress to the representational challenges identified in qualitative research, but also provides a deeper and more genuine understanding of the person - as his or her inner outlook.

Personal Construct Psychology embodies - in principle - the idiographic conceptualisation of being that is sought by Hermeneutic Constructivism. However the way that Kelly (1955) dealt poorly with language leaves open to challenge the genuinely reflexive nature of his theoretical position. Although the construct represented for Kelly (1955) the fundamental ontological structure of being, his situating of the construct beyond the reach of language comes to represent an untenable form of solipsism. In other words, the construct represents the core structure of the Self but being situated outside of language the construct, and therefore the Self, cannot begin to come to language without a subscription to a contestable correspondence between the construct and words. Such a subscription, however, represents a contradiction of Kelly's (1955) theoretical position. This thesis has therefore emphasised that the unsophisticated way that Kelly (1955) dealt with language, and perhaps 
more specifically the way that he was unequivocal that the construct cannot come to language, has implications for the claims to realism and indeed the theoretical utility that can be made by the Psychology of Personal Constructs. At the core of these implications are challenges to the extent to which real people can be genuinely represented by Kelly's (1955) theoretical position. Worded another way, if the construct does not lend itself to what can be brought to language by a person, then what is in fact being extracted about another person in the application of personal constructivist approaches? Despite the theoretical challenges levelled at Personal Construct Psychology several core principles embodied by Kelly's (1955) work, as well as his theoretical humility, have provided fertile ground for an elaboration and theoretical redress in the direction of taking greater account of the role of language.

Hermeneutic Constructivism represents the end result of a theoretical redress of the principles of Personal Construct Psychology from the philosophical vantage point of Hermeneutic Phenomenology. At the core of Gadamer's (1960/2003) Hermeneutic Phenomenology is a recognition that all being that can be understood is that which can come to language. This position represents the theoretical vantage point that informed this reenvisioning of the principles of Personal Construct Psychology and the subsequent setting down of Hermeneutic Constructivism. As a beginning point the Fundamental Postulate of Hermeneutic Constructivism was emphasised in the following way.

'A person's understanding processes are guided by his or her languaging of events' Elaborating this position by way of eleven subsequent corollaries, Hermeneutic Constructivism provides a theoretical conceptualisation of the way that the person goes about the business of being human. At its core Hermeneutic Constructivism emphasises that the comportment of being human is always toward understanding. Here each person develops a series of highly nuanced prejudices by way of which the world is able to come to understanding. Through the dialogue of languaging each person projects a prejudice toward the world in seeking to bring it to understanding. As such these fundamentally ontological structures are the essential precondition of all understanding, including a person's innermost experiences or qualia. The prejudice is therefore the foundation of the way in which we have a world at all.

The prejudice is synonymous with the Mot Juste, or the most perfect word or phrase available to a person in order to bring the world to understanding. The inextricability of the prejudice and those aspects of authentic consciousness that it opens up towards in the dialogue of languaging, means that the person of Hermeneutic Constructivism is able to be articulate about these structures to his or her self as well as to other people. As the epitome of 
the human comportment towards understanding, a person's Mot Juste always evolves towards a better or different understanding through the movement of languaging itself. Being commensurate with the level of the Self, the illumination of the Mot Juste therefore provides a most compelling inner outlook for the purpose of qualitative research. All this culminates in a view that at its core Hermeneutic Constructivism can be considered an attempt to contribute not only to the collective thinking about the functioning of the individual, but to represent more deeply that functioning, even if just for a short while.

Finally there is no intention to raise Hermeneutic Constructivism to a level of sovereignty within the domain of qualitative research. However the theoretically reflexive consideration of Person the Languager, and the elaboration of the prejudice as the medium of internal experience, means that the illumination of these structures is genuinely representative of the real people and real lives encountered in qualitative research. To this extent a representation of the person at this level of abstraction patently accommodates the challenges levelled at contemporary qualitative research throughout this thesis. In addition, the redress of these theoretical challenges by Hermeneutic Constructivism is commensurate with the core principles that are inherent within the notions of: validity, reliability and generalisability. In fact, it is anticipated that if the discipline of qualitative research is able to allow itself to come down from the mantle of being 'expert narrator', in order to refocus on the task of considering alternate approaches that may genuinely advance our understanding of what it means to be human, it will find Hermeneutic Constructivism a most agreeable forebear. The prospects for the future application of Hermeneutic Constructivism have indeed captured the enthusiasm of at least one languaging individual. 


\section{REFERENCES}

Adams-Webber, J. (1990). Personal construct theory and cognitive science. International Journal of Personal Construct Psychology, 3, 415-421.

Allport, G. W. (1962). The general and the unique in psychological science. Journal of Personality, 30, 405-422.

Altheide, D., \& Johnson, J. (1994). Criteria for assessing interpretive validity in qualitative research. In N. Denzin, \& Y. Lincoln (Eds.), Handbook of qualitative research (pp. 485-499). London: Sage.

Anderson, J. (1962). Studies in empirical philosophy. Sydney, Australia: Angus and Robertson.

Anderson, R. (1998). Intuitive inquiry: A transpersonal approach. In W. Braud, \& R. Anderson (Eds.), Transpersonal research methods for the social sciences: Honoring human experience (pp. 69-95). Thousand Oaks: Sage Publications.

Androutsopoulou, A. (2001). The self-charcterization as a narrative tool: Application in therapy with individuals and families. Family Process, 40(1), 79-94.

Angen, M. J. (2000). Pearls, pith and provocation: Evaluating interpretive inquiry: Reviewing the validity debate and opening the dialogue. Qualitative Health Research, 10(3), 378395.

Angus, Levitt and Hardke (1999). The narrative process coding system: research applications and implications for psychotherapy practice. Journal of Clinical Psychology, 55(10), 1255-1270.

Applegate, J. L. (1990). Constructs and communication: A pragmatic integration. In R. A. Neimeyer \& G. J. Neimeyer (Eds.), Advances in personal construct psychology (Vol. 1, pp. 203-230). Connecticut: JAI Press.

Apter, T. (1996). Expert witness: Who controls the psychologist's narrative? In R. Josselson (Ed.), The narrative study of lives: Vol. 4. ethics and process in the narrative study of lives (pp. 22-44). Thousand Oaks: Sage. 
Aranda, G., \& Finch, E. (2003). Using repertory grids to measure changes in risk-taking behavior. Journal of Construction Research, 4(1), 101-114.

Arendt, H. (1958). The human condition (2nd ed.). Chicago: University of Chicago Press.

Atkinson, P., \& Delamont, S. (2006). Rescuing narrative from qualitative research. Narrative Inquiry, 16(1), 164-172.

Atkinson, P., \& Silverman, D. (1997). Kundera's immortality: The interview society and the invention of the self. Qualitative Inquiry, 3(3), 304-325.

Bakan, D. (1996). Some reflections about narrative research and hurt and harm. In R. Josselson (Ed.), The narrative study of lives (Vol. 4, pp. 3-8). Thousand Oaks: Sage.

Bannister, D., \& Fransella, F. (1971). Inquiring man. Harmondsworth: Penguin.

Bannister, D., \& Mair, J. M. M. (1968). The evaluation of personal constructs. London: Academic Press.

Barbour, R. S. (2001). Checklists for improving rigour in qualitative research: A case of the tail wagging the dog. British Medical Journal, 322(7294), 1115-1117.

Barbu, Z. (1956). Democracy and dictatorship. London: Routledge.

Barker, M. (2003). Assessing the 'quality' in qualitative research. European Journal of Communication, 18(3), 315-335.

Barlow, D. H., \& Nock, M. K. (2009). Why cant we be more idiographic in our research? Perspectives on Psychological Science, 4(1), 19-21.

Bar-On, D. (1996). Ethical issues in biographical interviews and analysis. In R. Josselson (Ed.), The narrative study of lives: Vol. 4. ethics and process in the narrative study of lives (pp. 9-21). Thousand Oaks: Sage.

Baumeister, R. F. (1998). The self (4th ed.). New York: McGraw-Hill Publishing. 
Berger, P. L., \& Luckmann, T. (1966). The social construction of reality: A treatise in the sociology of knowledge. New York: Random House.

Bernstein, R. (1983). Beyond objectivism and relativism: Science, hermeneutics, an practice. Oxford: Blackwell Publishing.

Berzonsky, M. D. (1990). Self-construction over the lifespan: A process perspective on identity formation. In G. J. Neimeyer, \& R. A. Neimeyer (Eds.), Advances in personal construct theory (pp. 155-186). Greenwich: JAI Press.

Bloor, M. (1983). Notes on member validation. In R. M. Emerson (Ed.), Contemporary field research: A collection of readings (pp. 156-172). Boston: Little Brown.

Bloor, M. (1997). Techniques of validation in qualitative research: A critical commentary. In G. Miller, \& R. Dingwell (Eds.), Context and method in qualitative research (pp. 3750). London: Sage.

Blumfield-Jones, D. (1995). Fidelity as a criterion for practicing and evaluating narrative inquiry. International Journal of Qualitative Studies in Education, 8(1), 25-35.

Botella, L., \& Herrero, O. (2000). A relational constructivist approach to narrative therapy. European Journal of Psychotherapy, Counselling and Health, 3, 407-418.

Bourdieu, P. (1999). Understanding. In P. Bourdieu (Ed.), The weight of the world: Social suffering in contemporary society (pp. 607-626). London: Stanford University Press.

Bourdieu, P. (1999). The weight of the world: Social suffering in contemporary society. London: Stanford University Press.

Brabeck, M. M. (2000). Practicing feminist ethics in psychology. Washington, DC.: American Psychological Association.

Braun, V., \& Clarke, V. (2006). Using thematic analysis in psychology. Qualitative Research in Psychology, 3, 77-101. 
Briggs, C. L. (2002). Interviewing, power/knowledge, and social inequality. In J. F. Gubrium, \& J. A. Holstein (Eds.), Handbook of interview research (pp. 911-922). London, Thousand Oaks: Sage Publications.

Brinkmann, S. (2004). The topology of moral ecology. Theory and Psychology, 14(1), 57-80.

Brinkmann, S. (2007). Could interviews be epistemic? An alternative to qualitative opinion polling. Qualitative Inquiry, 13(8), 1116-1138.

Brinkmann, S., \& Kvale, S. (2005). Confronting the ethics of qualitative research. Journal of Constructivist Psychology, 18, 157-181.

Bryman, A., \& Cassell, C. (2006). The research interview: A reflexive perspective. Qualitative Research in Organizations and Management: An International Journal, $1(1), 41-55$.

Bugental, J. F. T. (1980). Being levels of therapeutic growth. In R. N. Walsh, \& F. Vaughan (Eds.), Beyond ego (pp. 195). Los Angeles: J.P.Tarcher.

Bugental, J. F. T., \& Bugental, E. K. (1984). A fate worse than death: The fear of changing. Psychotherapy, 21, 543-549.

Burke, K. (1966). Language as symbolic action: Essays on life, literature, and method. Los Angeles: University of California.

Burkitt, I. (1996). Social and personal constructs: A division left unresolved. Theory and Psychology, 6(1), 71-77.

Burman, E. (1997). Minding the gap: Positivism, psychology, and the politics of qualitative methods. Journal of Social Issues, 53, 785-801.

Butler, R., \& Green, D. (1998). The child within: The exploration of personal construct theory with young children. Oxford: Butterworth-Heinemann.

Butler, R. J. (1994). Nocturnal enuresis: The child's experience. Oxford: ButterworthHeinemann. 
Butler, R. J. (2001). The self image profiles: Manual. London: The Psychological Corporation.

Butler, R. J. (2006). Investigating the content of core constructs. Personal Construct Theory and Practice, 3, 27-33.

Butt, T. (1995). Ordinal relationships between constructs. Journal of Constructivist Psychology, 8(3), 227-236.

Butt, T. (1996). PCP: Cognitive or social psychology? In J. W. Scheer (Ed.), Empirical constructivism in europe (pp. 58-67). Giessen, Germany: Psychosozial-Verlag.

Butt, T. (1997a). The existentialism of George Kelly. Journal of the Society for Existential Analysis, 8, 20-32.

Butt, T. (1997b). The later Kelly and existential phenomenology. In P. M. Denicolo, \& M. L. Pope (Eds.), Sharing understanding and practice (pp. 40-49). Farnborough: EPCA Publications.

Butt, T. (1998). Sociality, role and embodiment. Journal of Constructivist Psychology, 11, 105-116.

Butt, T. (1999). Realism, constructivism and phenomenology. In D. J. Nightingale, \& J. Cromby (Eds.), Social constructionist psychology: A critical analysis of theory and practice (pp. 127-140). Buckingham: Open University Press.

Butt, T. (2000). Construct psychology and social action. In J. W. Scheer (Ed.), The person in society: Challenges to a constructivist theory (pp. 176-185). Giessen, Germany: Psychosozial-Verlag.

Butt, T. (2000a). Pragmatisim, constructivism, and ethics. Journal of Constructivist Psychology, 13 85-101.

Butt, T. (2001). Social action and personal constructs. Theory and Psychology, 11(1), 75-95. 
Butt, T. (2003). The phenomenological context of personal construct psychology. In F. Fransella (Ed.), International handbook of personal construct psychology (pp. 379386). Chichester, England: John Wiley and Sons.

Butt, T. (2004a). Understanding people. Hampshire, England: Palgrave Macmillan.

Butt, T. (2004b). Understanding, explanation, and personal constructs. Personal Construct Theory and Practice, 1, 21-27.

Butt, T. (2005). Personal construct theory, phenomenology and pragmatism. History and Philosophy of Psychology, 7(1), 23-35.

Butt, T. (2008). Georg Kelly: The psychology of personal constructs. Houndmills, London: Palgrave Macmillan.

Butt, T., Burr, V., \& Bell, R. (1997). Fragmentation and the sense of self. Constructivism in the Human Sciences, 2, 12-29.

Butt, T., \& Burr, V. (2009). Making the most of methods: the future for PCP? In: The XVIIIth International Congress on Personal Construct Psychology, July 20-24 2009, Venice, Italy.

Charmaz, K. (2004). Premises, principles, and practices in qualitative research: Revisiting the foundations. Qualitative Health Research, 14(7), 976-993.

Chase, S. E. (1996). Personal vulnerability and interpretative authority in narrative research In R. Josselson (Ed.), The narrative study of lives: Vol. 4. ethics and process in the narrative study of lives (pp. 45-59). Thousand Oaks: Sage.

Chiari, G. (2000). Personal construct theory and the constructivist family: A friendship to cultivate, a marriage not to celebrate. In J. W. Scheer (Ed.), The person in society: Challenges to a constructivist theory. (pp. 66-78). Giessen, Germany: PsychosozialVerlag.

Chiari, G., \& Nuzzo, L. (1996a). Personal construct theory within psychological constructivism: Precursor or avant-garde? In B. M. Walker, J. Costigan, L. L. Viney \& 
B. Warren (Eds.), Personal construct theory: A psychology for the future (pp. 25-54).

Sydney, Australia: The Australian Psychological Society.

Chiari, G., \& Nuzzo, L. (1996b). Psychological constructivisms: Metatheoretical differentiation. Journal of Constructivist Psychology, 9, 163-184.

Chiari, G., \& Nuzzo, L. (2004). Steering personal construct theory towards a hermeneutic constructivism. In J. D. Raskin, \& S. K. Bridges (Eds.), Studies in meaning 2: Bridging the personal and social in constructivist psychology (pp. 51-65). New York: Pace University Press.

Chiari, G., \& Nuzzo, M. L. (1988). Embodied minds over interacting bodies: A constructivist perspective on the mind-body problem. The Irish Journal of Psychology, 9, 91-100.

Chiari, G., \& Nuzzo, M. L. (2000). Hermeneutics and constructivist psychotherapy: The psychotherapeutic process in a hermeneutic constructivist framework. In J. W. Scheer (Ed.), The person in society: Challenges to a constructivist theory. (pp. 90-99). Giessen, Germany: Psychosozial-Verlag.

Chiari, G., \& Nuzzo, M. L. (2006). Exploring the sphere of between: The adoption of a framework of complementarity and its implications for constructivist psychotherapy. Theory and Psychology, 16(2), 257-275.

Chiari, G., \& Nuzzo, M. L. (2003). Kelly's philosophy of constructive alternativism. In F. Fransella (Ed.), International handbook of personal construct psychology. (pp. 41-49). San Francisco, CA: John Wiley and Sons.

Chiari, G., \& Nuzzo, M.L.(2003a) Psychological constructivism and the social world. Milan: Franco Angeli Publishing.

Chiari, G., \& Nuzzo, M. L. (2010). Constructivist psychotherapy: A narrative approach. New York: Routledge, Taylor and Francis Group.

Cho, J., \& Trent, A. (2006). Validity in qualitative research revisited. Qualitative Research, 6(3), 319-340. 
Christians, C. (2000). Ethics and politics in qualitative research. In N. K. Denzin, \& Y. S. Lincoln (Eds.), Handbook of qualitative research (2nd ed., pp. 133-155). Thousand Oaks: Sage.

Clavarino, A., Najman, J., \& Silverman, D. (1995). Assessing the quality of qualitative data. Qualitative Inquiry, 1(2), 223-242.

Coffey, A. (1999). The ethnographic self: Fieldwork and the representation of identity. London: Sage.

Cohen, M. (2005). Wittgenstein's beetle and other classic thought experiments. Oxford: Blackwell Publishing.

Colaizzi, P. (1978). Psychological research as a phenomenologist views it. In R. S. Valle, \& M. King (Eds.), Existential-phenomenological alternatives for psychology (pp. 48-71). New York: Oxford University Press.

Colapietro, V. M. (1990). The vanishing subject of contemporary discourse. Journal of Philosophy, 87(11), 644-655.

Cole, C. (1991). The politics of cultural representation: Vision of fields / fields of vision. International Review for the Sociology of Sport, 26(1), 36-49.

Coogan, M. (Ed.). (2001). The new oxford annotated bible (3rd ed. NRSV ed.). New York: Oxford University Press.

Costigan, J., Closs, B., \& Eustace, P. (2000). Laddering: Theoretical methodological contingencies - some order and a little chaos. In J. W. Scheer (Ed.), The person in society : Challenges to a constructivist theory (pp. 150-159). Giessen, Germany: Psychosozial-Verlag.

Cox, L. M., \& Lyddon, W. J. (1997). Constructivist conceptions of self: A discussion of emerging identity constructs. Journal of Constructivist Psychology, 10, 201-220.

Crockett, W. H. (1965). Cognitive complexity and impression formation. In B. A. Maher (Ed.), Progress in experimental personality research (Vol 2, pp.47-90). New York: Academic Press. 
Crockett, W. H. (1982). The organization of construct systems: The organization corollary. In J. C. Mancuso, \& J. R. Adams-Weber (Eds.), The construing person (pp. 62-95). New York: Praeger.

Datan, N., Rodeheaver, D., \& Hughes, F. (1987). Adult development and aging. Annual Review of Psychology, 38, 153-180.

Davidson, G., \& Reser, J. (1996). Construing and constructs: Personal and cultural? In B. M. Walker, J. Costigan, L. L. Viney \& B. Warren (Eds.), Personal construct theory. A psychology for the future (pp. 105-128). Melbourne, Australia.: APS Imprint.

de Saussure, F. (1974). Course in general linguistics (F. Pogson Trans.). London: Allen and Unwin.

Denicolo, P. (2003). Elicitation methods to fit different purposes. In F. Fransella (Ed.), International handbook of personal construct psychology (pp. 123-132). London: John Wiley and Sons.

Dennett, D. (1988). Quining qualia. In A. Marcel, \& E. Bisiach (Eds.), Consciousness in modern science (pp. 11-21). London: Oxford University Press.

Denzin, N., \& Lincoln, Y. (1994). Introduction: Entering the field of qualitative research. In N. Denzin, \& Y. Lincoln (Eds.), Handbook of qualitative research (pp. 1-17). London: Sage Publications.

Denzin, N., \& Lincoln, Y. S. (2005). The sage handbook of qualitative research (3rd ed.). Thousand Oaks: Sage.

Denzin, N. K. (2001). The reflexive interview and a performative social science. Qualitative Research, 1(1), 23-46.

Deutscher, M. (1983). Subjecting and objecting. St.Lucia, Brisbane, Australia: University of Queensland Press.

Dinkins, C. S. (2005). Shared inquiry: Socratic-hermeneutic interpre-viewing. In P. Ironside (Ed.), Beyond method: Philosophical conversations in healthcare research and scholarship (pp. 111-147). Madison: University of Wisconsin Press. 
Dixon-Woods, M., Bonas, S., Booth, A., Jones, D. R., Miller, T., Sutton, A. J., \& Young, B. (2006). How can systematic reviews incorporate qualitative research? A critical perspective. Qualitative Research, 6(1), 27-44.

Dixon-Woods, M., Shaw, R. L., Agarwal, S., \& Smith, J. A. (2004). The problem of appraising qualitative research. Quality and Safety in Health Care, 13, 223-225.

Domenici, D. J. (2008). Implications of hermeneutic constructivism for personal construct theory: Imaginally construing the nonhuman world. Journal of Constructivist Psychology, 21(1), 25-42.

Downs, Y. (2010). Transcription tales or let not love's labour be lost. International Journal of Research and Method in Education, 33(1), 101-112.

Duck, S. W. (1994). Meaningful relationships: Talking, sense, and relating. London: Sage Publications.

Duncombe, J., \& Jessop, J. (2002). "Doing rapport" and the ethics of "faking friendship.". In M. Mauther, M. Birch, J. Jessop \& T. Miller (Eds.), Ethics in qualitative research (pp. 107-122). London: Sage Publications.

Efran, J. S., \& Clarfield, L. E. (1992). Constructionist therapy: Sense and non-sense. In S. McNamee, \& K. J. Gergen (Eds.), Therapy as social construction (pp. 200-217). Thousand Oaks: Sage.

Eisner, E. W. (2003). On the art and science of qualitative research in psychology. In P.M. Camic, J.E. Rhodes, \& L. Yardley (Eds.), Qualitative research in psychology: Expanding perspectives in methodology and design (pp. 17-29). Washington, DC: American Psychological Association.

Elliot, R., Fischer, C., \& Rennie, D. (1999). Evolving guidelines for publication of qualitative research studies in psychology and related fields. British Journal of Clinical Psychology, 38, 215-229.

Ellis, C. (1997). Evocative autoethnography: Writing emotionally about our lives. In W. G. Tierney, \& Y. S. Lincoln (Eds.), Representation and the text: Re-framing the narrative voice (pp. 115-139). New York: State University of New York Press. 
Ellis, C. (1999). Heartful autoethnography. Qualitative Health Research, 9, 669-683.

Epting, F. R. (1988). Journeying into the personal constructs of children. International Journal of Personal Construct Psychology, 1, 53-61.

Epting, F. R., Suchman, D. I., \& Nickeson, C. J. (1971). An evaluation of elicitation procedures for personal constructs. British Journal of Psychology, 62(4), 513-517.

Feixas, G. (1995). Personal constructs in systematic practice. In R. A. Neimeyer, \& M. J. Mahoney (Eds.), Constructivism in psychotherapy (pp. 305-337). Washington, DC: American Psychological Association.

Feixas, G., Bach, L., \& Laso, E. (2004). Factors affecting interpersonal construct differentiation when measured using the repertory grid. Journal of Constructivist Psychology, 17, 297-311.

Feltham, C. (2010). Critical thinking in counselling and psychotherapy. Thousand Oaks: Sage Publications.

Ferudi, F. (2004). Therapy culture: Cultivating vulnerability in an uncertain age. London: Routledge.

Figal, G. (2002). The doing of the thing itself: Gadamer's hermeneutic ontology of language. In R. J. Dostal (Ed.), The Cambridge companion to Gadamer (pp. 102-125). Cambridge: Cambridge University Press.

Fine, M. (1994). Dis-stance and other stances: Negotiations of power inside feminist research. In A. Gitllin (Ed.), Power and method: Political activism and educational research (pp. 13-35). New York: Routledge.

Fish, W. (2010). Philosophy of perception: A contemporary introduction. New York: Routledge.

Fisher, J.M. (2003). Fraggle rock: A study in isolationism. In G. Chiari \& M. Nuzzo (Eds.), Psychological constructivism and the social world (pp. 98-104). Milan: Franco Angeli Publishing. 
Flyvbjerg, B. (2004). Five misunderstandings about case-study research. In C. Seale, G.

Gobo, J. Gubrium \& D. Silverman (Eds.), Qualitative research practice (pp. 420-434). London: Sage.

Fog, J. (2004). Med samtalen som udgangspunkt [with the conversation as basis].

Copenhagen: Akademisk Forlag.

Frank, M. (1989). What is neostructuralism? (S. Wilke, R. Gray Trans.). Minneapolis: University of Minnesota Press.

Fransella, F. (1981). Nature babbling to herself: The self characterisation as a psychotherapeutic tool. In H. Bonarius, R. Holland \& S. Rosenberg (Eds.), Personal construct psychology: Recent advances in theory and practice (pp. 219-230). London: Macmillan.

Fransella, F. (1989). What is a construct? In D. A. Lane (Ed). Attributes, beliefs and constructs in counseling psychology, Occasssional paper, Counseling Section BPS, (pp. 21-25). Leicester: British Psychological Society.

Fransella, F. (1995). George Kelly. London: Sage Publications.

Fransella, F. (2003). Some skills and tools for personal construct practitioners. In F. Fransella (Ed.), International handbook of personal construct psychology (pp. 105-122). Chichester, London: John Wiley and Sons.

Fransella, F., \& Neimeyer, R. A. (2003). George Alexander Kelly: The man and his theory. In F. Fransella (Ed.), International handbook of personal construct psychology (pp. 2132). Chichester, England: John Wiley and Sons.

Fransella, F., \& Thomas, L. F. (1988). Experimenting with personal construct psychology. London: Routledge \& Kegan Paul.

Freeman, M., deMarrais, K., Preissle, J., Roulston, K., \& Pierre, E. A. (2007). Standards of evidence in qualitative research: An incitement of discourse. Educational Researcher, $36(1), 25-32$.

Freud, S. (1963). Therapy and technique. New York: Collier Books. 
Fry, G., Butt, T., \& Bell, R. (2003). Joint action and core construing. In G. Chiari \& M. Nuzzo (Eds.), Psychological constructivism and the social world (pp.67-78). Milan: Franco Angeli Publishing.

Fuchs, M. (1993). The reversal of the ethnological perspective: Attempts at objectifying one's own cultural horizon. Dumont, Foucalt, Bourdieu? Thesis Eleven, 34, 104-125.

Fuentes, L. (2004). Ethics in violence against women research: The sensitive, the dangerous, and the overlooked. Ethics and Behavior, 14, 141-174.

Gadamer, H. G. (1976). Hegel's dialectic: Five hermeneutical studies (C. Smith Trans.). New Haven: Yale University Press.

Gadamer, H. G. (1977). Philosophical hermeneutics (D. E. Linge Trans.). Los Angeles: University of California Press.

Gadamer, H. G. (2003). Truth and method (J. Weinsheimer, D. Marshall Trans.). (2nd Revised Edition.). New York: Continuum Press.

Gallagher, D. (1995). In search of the rightful role of method: Reflections on conducting a qualitative dissertation. In T. Tiller, A. Sparkes \& F. Dowling Naess (Eds.), The qualitative challenge (pp. 25-43). Landas, Norway: Caspar Forlag.

Gallagher, D. J. (2001). Neutrality as a moral standpoint. Conceptual confusion and the full inclusion debate. Disability and Society, 16(5), 637-654.

Garratt, D., \& Hodkinson, P. (1998). Can there be criteria for selecting research criteria? A hermeneutical analysis of an inescapable dilemma. Qualitative Inquiry, 4, 515-539.

Geertz, C. (1973). The interpretation of cultures. New York: Basic Books.

Gergen, K. J. (1985). Social constructionist inquiry: Context and implications. In K. J.

Gergen, \& K. E. Davies (Eds.), The social construction of the person. (pp. 3-18). New York.: Springer.

Gergen, K. J. (1985). The social constructionist movement in modern psychology. American Psychologist, 40, 266-275. 
Gergen, K. J. (1994). Realities and relationships: Soundings in social construction. Cambridge, Massachusetts: Harvard University Press.

Gergen, K. J., McNamee, S., \& Barrett, F. (2001). Toward transformative dialogue. International Journal of Public Administration, 24(7,8), 679-707.

Gergen, M. M., \& Gergen, K. J. (2000). Qualitative inquiry tensions and transformations. In N. K. Denzin, \& Y. S. Lincoln (Eds.), The handbook of qualitative research (2nd ed., pp. 1025-1046). Thousand Oaks: Sage Publications.

Gillett, E. (1998). Relativism and the social constructivist paradigm. Philosophy, Psychiatry and Psychology, 5, 37-48.

Giorgi, A. (1985). Sketch of a psychological phenomenological method. In A. Giorgi (Ed.), Phenomenology and psychological research. (pp. 8-22). Pittsburgh: Duquesne University Press.

Glesne, C., \& Peshkin, A. (1992). Becoming qualitative researchers: An introduction. White Plains, New York: Longman.

Golafshani, N. (2003). Understanding reliability and validity in qualitative research. The Qualitative Report, 8(4), 597-606.

Goncalves, O. F. (1994). Cognitive narrative psychotherapy: the hermeneutic construction of alternative meanings. Journal of Cognitive Psychotherapy, 8, 105-126.

Goncalves, O. F. (1995). Hermeneutics, constructivism, and cognitive-behavioural therapies: from the object to the project. In R. A. Neimeyer \& M. J. Mahoney (Eds.), Constructivism in Psychotherapy. (pp. 195-230). Washington, DC: American Psychological Assosciation.

Goodrich, R. A. (1993). Deconstructing constructs: pitfalls in personal construct theory. Educational Philosophy and Theory, 25(1), 71-82.

Green, J., Franquiz, M., \& Dixon, C. (1997). The myth of the objective transcript: Transcribing as a situated act. TESOL Quarterly, 31(1), 172-176. 
Grondin, J. (2003). The philosophy of Gadamer (K. Plant Trans.). Montreal: McGill-Queens University Press.

Guba, E. G., \& Lincoln, Y. S. (2005). Paradigmatic controversies, contradictions, and emerging confluences. In N. K. Denzin, \& Y. S. Lincoln (Eds.), The sage handbook of qualitative research (3rd ed., pp. 191-216). London: Sage.

Gubrium, J. F., \& Holstein, J. A. (2002). From the individual interview to the interview society. In J. F. Gubrium, \& J. A. J Holstein (Eds.), Handbook of interview research: Context and methods (pp. 3-32). Thousand Oaks: Sage.

Gubrium, J. F., \& Holstein, J. A. (2002). Handbook of interview research: Context and method. Thousand Oaks: Sage.

Guidano, V. F. (1987). Complexity of self: A developmental approach to psychotherapy and therapy. New York: The Guilford Press.

Guidano, V. F. (1991). The self in process: Toward a post-rationalist cognitive therapy. New York: The Guilford Press.

Guidano, V. F. (1995). A constructivist outline of human knowing processes. In M. J. Mahoney (Ed.), Cognitive and constructive psychotherapies: Theory research and practice (pp. 89-102). New York: Springer Publishing Company.

Guillemin, M., \& Gillam, L. (2004). Ethics, reflexivity, and "ethically important moments" in research. Qualitative Inquiry, 10, 261-280.

Hadjistavropoulos, T., \& Smythe, W. E. (2001). Elements of risk in qualitative research. Ethics and Behavior, 1(2), 163-174.

Hagans, C. L., Neimeyer, G. J., \& Goodholm, R. (. (2000). The effect of elicitation methods on personal construct differentiation and valence. Journal of Constructivist Psychology, $13,155-173$

Hamann, J. G. (1949-1957). Samtliche werke. Vienna: Herder.

Hamann, J. G. (1967). In Joset Simon (Ed.), Schriften zuf sprache. Frankfurt: Suhrkamp. 
Hammersley, M. (1990). Reading ethnographic research: A critical guide. London: Longman.

Hammersley, M. (1991). A note on Campbell's distinction between internal and external validity. Quality and Quantity, 25(4), 381-387.

Hammersley, M. (1992). What is wrong with ethnography? methodological explorations. London: Routledge.

Hammersley, M. (1995). Theory and evidence in qualitative research. Quality and Quantity, 29, 55-66.

Hammersley, M. (1998). Telling tales about educational research: A response to John.K. Smith. Educational Researcher, 27(18), 18-21.

Hammersley, M. (1999). Some reflections on the current state of qualitative research. Research Intelligence, 70, 16-187.

Hammersley, M. (2005). Should social science be critical? Philosophy of the Social Sciences, $35,175-195$.

Hammersley, M. (2008). Assessing validity in social science research. In P. Alasuutari, L. Bickman \& J. Brannen (Eds.), The Sage handbook of social science research methods (pp. 42-53). London: Sage.

Hammersley, M. (2009a). Challenging relativism: The problem of assessment criteria. Qualitative Inquiry, 15(1), 3-29.

Hammersley, M. (2009b). Closing down the conversation? A reply to Smith and Hodkinson. Qualitative Inquiry, 15(1), 40-48.

Hardison, H. G. \& Neimeyer, R. A. (2007). Numbers and narratives: quantitative and qualitative convergence across constructivist assessments. Journal of Constructivist Pychology, 20, 285-308.

Hare-Mustin, R. T. (1994). Discourses in the mirrored room: A postmodern analysis of therapy. Family Process, 33, 19-35. 
Harre, R. (1989). Metaphysics and methodology: Some prescriptions for social psychological research. European Journal of Social Psychology, 19, 439-453.

Harre, R. (2006). Key thinkers in psychology. London: Sage Publications.

Harre, R., \& Gillett, G. (1994). The discursive mind. London: Sage Publications.

Harre, R., \& Tissaw, M. (2005). Wittgenstein and psychology: A practical guide. Burlington, USA: Ashgate Publishing Company.

Have, P. (2004). Understanding qualitative research and ethnomethodology. London: Sage Publications.

Hegel, G. W. F. (1807/1967). The phenomenology of mind (J. B. Baillie Trans.). New York: Harper.

Heidegger, M. (2005). Being and time (J. Macquarrie, E. Robinson Trans.). London: Blackwell Publishing.

Heil, J. (2004). Philosophy of mind: A contemporary introduction (2nd ed.). London: Routledge, Taylor and Francis Group.

Held, B. S. (1995a). Back to reality: A critique of postmodern theory in psychotherapy. New York: W.W. Norton and Company.

Held, B. S. (1995b). The real meaning of constructivism. Journal of Constructivist Psychology, 8, 305-316.

Held, B. S. (1996). Constructivism in psychotherapy: Truth and consequences. Annals of New York Academy of Sciences (the Flight from Science and Reason), 775, 198-206.

Held, B. S. (1998). The many truths of postmodernist discourse. Journal of Theoretical and Philosophical Psychology, 18, 193-217.

Herder, J. G. v. (1972/2002). On the origin of language [Herder: Philosophical Writings] (M. Forster Trans.). Cambridge: Cambridge University Press. 
Hermans, H. J. M. (1999). Self-narrative as meaning construction: The dynamics of selfinvestigation. Journal of Clinical Psychology, 55(10), 1193-1211.

Hermans, H. J. M. (2002). The dialogical self as a society of mind. Theory of Psychology, 12, 147-160.

Hermans, H. J. M. (2002a). The person as a Motivated storyteller: Valuation, theory and the self-confrontation method. In R.A. Neimeyer \& G. J. Neimeyer (Eds.), Advances in personal construct psychology: New directions and perspectives (pp. 3-38). Westport CT: Praeger.

Hermans, H. J. M. (2003). The construction and reconstruction of a dialogical self. Journal of Constructivist Psychology, 16, 89-130.

Hermans, H. J. M., Kempen, H. J. G., \& Van Loon, R. J. P. (1992). The dialogical self: beyond individualism and rationalism. American Psychologist, 47, 23-33.

Hinkle, D. N. (1965). The change of personal constructs from the view point of a theory of construct implications. Unpublished manuscript.

Hinkle, D. N. (2010). A brief glance backward. Personal Construct Theory and Practice, 7(Supplement No.1), vii-ix.

Ho, D. Y. F., Ho, R. T. H., \& Ng, S. M. (2007). Restoring quality to qualitative research. Culture and Psychology, 13(3), 377-383.

Hodkinson, P. (2004). Research as a form of work: Expertise, community and methodological objectivity. British Educational Research Journal, 30(1), 9-26.

Holland, J. M., Neimeyer, R. A., Currier, J. M., \& Berman, J. S. (2007). The efficacy of personal construct therapy: A comprehensive review. Journal of Clinical Psychology, 63(1), 93-107.

Holland, R. (1970). George Kelly: Constructive innocent and reluctant existentialist. In D. Bannister (Ed.), Perspectives in personal construct theory (pp. 111-132). London: Academic Press. 
Holloway, I., Todres, L. (2003). The status of method: Flexibility, consistency and coherence. Qualitative Research, 3, 345-357.

Horsburgh, D. (2003). Evaluation of qualitative research. Journal of Clinical Nursing, 12, 307-312.

Houston, J. (1998). Making sense with offenders. Personal constructs, therapy and change. Chichester, England: John Wiley and Sons.

Humbolt, W. v. (1988). On language. The diversity of human language - structure and its influence on the mental development of mankind. Cambridge: Cambridge University Press.

Hundert, E. M. (1995). Lessons from an optical illusion: On nature and nurture, knowledge and values. Cambridge: Harvard University Press.

Hunt, H. T. (Ed.). (2003). Lives as spirit: Precursors and dilemmas of a secular western mysticism. Albany: State University of New York Press.

Hurlburt, R. T., \& Knapp, T. J. (2006). Munsterberg in 1989, not Allport in 1937, introduced the terms 'idiographic' and 'nomothetic' to American psychology. Theory and Psychology, 16(2), 287-293.

Husain, M. (1983). To what can one apply a construct? In J. Adams-Webber, \& J. C. Mancuso (Eds.), Applications of personal construct psychology (pp. 11-28). New York: Academic Press.

Hyden, L. C. (1997). Illness and narrative. Sociology of Health and Illness, 19(1), 48-69.

Jackson, S. R. (1988). Self-characterisation: Dimensions of meaning. In F. Fransella, \& L. Thomas (Eds.), Experimenting with personal construct psychology (pp. 223-231). London: Routledge \& Kegan Paul.

Janesick, V. J. (2001). Intuition and creativity: A pas de deux for qualitative researchers. Qualitative Inquiry, 7(5), 531-540. 
Jankowicz, D. (2004). The easy guide to repertory grids. Chichester, England: John Wiley and Sons.

Johnson, J. L. (1997). Generalizability in qualitative research: Excavating the discourse. In J. M. Morse (Ed.), Completing a qualitative project: Details and dialogue (pp. 191-208). Thousand Oaks: Sage.

Jones, H. G. (1971). In search of an idiographic psychology, Bulletin of the BritishPsychological Society, 24, 279-290.

Jones, S. H. (2005). Autoethnography: Making the personal political. In N. K. Denzin, \& Y. S. Lincoln (Eds.), The sage handbook of qualitative research (3rd ed., pp. 763-793). London: Sage.

Joppe, M. (2000). The research process. Unpublished manuscript.

Josselson, R. (1996). On writing other people's lives: Self-analytic reflections of a narrative researcher. In R. Josselson (Ed.), The narrative study of lives: Vol.4. ethics and process in the narrative study of lives (pp. 60-71). Thousand Oaks, CA: Sage.

Kalekin-Fishman, D., \& Walker, B. (1996). The construction of group realities: Culture, society and personal construct psychology. Malabar, Florida: Krieger.

Karnieli-Miller, O., Strier, R., \& Pessach, L. (2009). Power relations in qualitative research. Qualitative Health Research, 19(2), 279-289.

Kastenbaum, R. J. (1985). The life history work of Barbara Myerhoff: A critical appraisal. Proceedings of the 38th Annual Meeting of Gerontological Society of America, New Orleans.

Kelly, G. A. (1955). The psychology of personal constructs. New York: W.W. Norton \& Company Inc.

Kelly, G. A. (1958). Man's construction of his alternatives. In B. Maher (Ed.), Clinical psychology and personality: The selected papers of George Kelly. (pp. 66-93). New York: John Wiley and Sons. 
Kelly, G. A. (1962). Europe's matrix of decision. Nebraska Symposium on Motivation, Nebraska.

Kelly, G. A. (1964). The language of hypothesis: Man's psychological instrument. In B. Maher (Ed.), Clinical psychology and personality: The selected papers of George Kelly (pp. 147-162). New York: John Wiley and Sons.

Kelly, G. A. (1966/1970). A brief introduction to personal construct theory. In D. Bannister (Ed.), Perspectives in personal construct theory (pp.1-30). London: Academic Press.

Kelly, G. A. (1969). Nonparametric factor analysis of personality theories. In B. Maher (Ed.), Clinical psychology and personality (pp. 301-332). New York: Wiley \& Sons.

Kelly, G. A. (1977). The psychology of the unknown. In D. Bannister (Ed.), New perspectives in personal construct theory. (pp. 1-19). London: Academic Press.

Kennedy, M. M. (1979). Generalizing from single case studies. Evaluation Quarterly, 3, 661678.

Kenny, A. J. P. (2006a). Wittgenstein (Revised Edition ed.). London: Blackwell Publishing.

Kenny, A. J. P. (2006b). The Wittgenstein reader (2nd ed.). London: Blackwell Publishing.

King, G., Keohane, R. O., \& Verba, S. (1994). Designing social inquiry: Scientific inference in qualitative research. New Jersey: Princeton University Press.

Kirk, J., \& Miller, M. (1986). Reliability and validity in qualitative research. London: Sage Publications.

Klevjer, I., \& Walker, B. (2002). Beyond the 'Big Five': A qualitative study of age differences in personality. Australian Journal of Psychology (Supplement), 54(5)

Knapik, M. (2006). The qualitative research interview: Participants' responsive participation in knowledge making. International Journal of Qualitative Methods, 5(3), 1-13.

Kogan, S. M. (1998). The politics of making meaning: Discourse analysis of a 'postmodern' interview. Journal of Family Therapy, 20, 229-251. 
Kreber, C. (2005). Reflections on teaching and the scholarship of teaching: Focus on science instructors. Higher Education, 50(2), 323-359.

Kvale, S. (1996). Interviews: An introduction to qualitative interviewing. Thousand Oaks: Sage Publications.

Kvale, S. (1999). The psychoanalytic interview as qualitative research. Qualitative Inquiry, 5(1), 87-113.

Kvale, S. (2003). The psychoanalytic interview as inspiration for qualitative research. In P. Camic, J. Rhodes \& L. Yardley (Eds.), Qualitative research in psychology: Expanding perspectives in methodology and design (pp. 275-297). Washington, DC: American Psychological Association Press.

Kvale, S. (2006). Dominance through interviews and dialogues. Qualitative Inquiry, 12(3), 480-500.

Kvale, S., \& Brinkmann, S. (2009). Interviews: Learning the craft of qualitative research interviewing (2nd ed.). Thousand Oaks: Sage Publications.

Lacan, J. (1977). Ecritis (A. Sheridan Trans.). London: Tavistock.

Lamiell, J. T. (1981). Toward an idiothetic psychology of personality. American Psychologist, 36, 276-289.

Lamiell, J. T. (1987). The psychology of personality: An epistemological inquiry. New York: Columbia University Press.

Lamiell, J. T. (1997). Individuals and the differences between them. In R. Hogan, J. A. Johnson \& S. R. Briggs (Eds.), Handbook of personality psychology (pp. 117-141). San Diego: Academic Press.

Lamiell, J. T. (1998). Nomothetic and idiographic: Contrasting Windelband's understanding with contemporary usage. Theory and Psychology, 8(1), 23-38. 
Lamiell, J. T. (2003). Beyond individual and group differences: Human individuality, scientific psychology, and William Stern's critical personalism. Thousand Oaks: Sage Publications.

Lamiell, J. T. (2007). On sustaining critical discourse with mainstream personality investigators: Problems and prospects. Theory and Psychology, 17(2), 169-185.

Lamiell, J. T. (2009a). Reviving person-centered inquiry in psychology: Why it's erstwhile dormancy? In J. Valsiner, M. C. Molenaar, P. Lyra \& N. Chaudhary (Eds.), Dynamic process methodology in the social and developmental sciences (pp. 31-43). London: Springer.

Lamiell, J. T. (2010). Reflections on some neglected ideas about psychological measurement from the personalistic perspective of William Stern (1871-1938). In A. Toomela, \& J. Valsiner (Eds.), Methodological thinking in psychology: 60 years gone astray? (pp. 189-208). New York: Information Age Publishing.

Landfield, A. W., \& Epting, F. R. (1987). Personal construct psychology: Clinical and personality assessment. New York: Human Sciences Press.

Lapadat, J. C., \& Lindsay, A. (1999). Transcription in research and practice: From standardization of technique to interpretive positioning. Qualitative Inquiry, 5, 64-86.

Lapadat, J. C. (2000). Problematizing transcription: Purpose, paradigm and quality. International Journal of Social Research Methodology, 3(3), 203-219.

Lather, P. (1993). Fertile obsession: Validity after poststructuralism. The Sociological Quarterly, 34, 673-693.

Latour, B. (2000). When things strike back: A possible contribution of "science studies" to the social sciences. British Journal of Sociology, 51(1), 107-123.

Lawn, C. (2004). Wittgenstein and Gadamer: Toward a post-analytic philosophy of language. London: Continuum Press.

Lee, S. D. (2001). The morning tea break ritual: A case study. International Journal of Nursing Practice, 7, 69-73. 
Leitner, L., \& Thomas, J. (2003). Experiential personal construct psychotherapy. In F. Fransella (Ed.), International handbook of personal construct psychology (pp. 257264). Chichester, England: John Wiley and Sons.

Leman, G. (1970). Words and worlds. In D. Bannister (Ed.), Perspectives in personal construct theory (pp. 133-156). London: Academic Press.

Lincoln, Y. (1993). I and thou: Method, voice, and roles in research with the silenced. In McLaughlin, D., Tierney, W. (Ed.), Naming silenced lives (pp. 29-47). New York: Routledge.

Lincoln, Y. S., \& Guba, E. G. (1985). Naturalistic inquiry. Beverly Hills, CA: Sage.

Mackay, N. (1994). Cognitive therapy, constructivist meta theory and rational explanation. Australian Journal of Psychology, 46(1), 7-12.

Mackay, N. (1997). Constructivism and the logic of explanation. Journal of Constructivist Psychology, 10(4), 339-361.

Mackay, N. (2003). Psychotherapy and the idea of meaning. Theory and Psychology, 13(3), 359-386.

Mair, M. (1970). Experimenting with individuals. British Journal of Medical Psychology, 43, 245-256.

Mair, M. (1988). Psychology as storytelling. International Journal of Personal Construct Psychology, 1, 125-137.

Mair, M. (1989). Kelly, Bannister, and a story-telling psychology. International Journal of Personal Construct Psychology, 2, 1-14.

Mair, M. (2000). Psychology as a discipline of discourse. European Journal of Psychotherapy, Counselling and Health, 3(3), 335-347.

Mancuso, J. C. (1996). The socializing of personal constructions. Theory and Psychology, $6(1), 85-92$. 
Manderson, L., Bennett, E., \& Andajani-Sutahjo, S. (2006). The social dynamics of the interview: Age, class and gender. Qualitative Health Research, 16(10), 1317-1334.

Manning, K. (1997). Authenticity in constructivist inquiry: Methodological considerations without prescription. Qualitative Inquiry, 3, 93-115.

Mantzoukas, S. (2004). Issues of representation within qualitative research. Qualitative Health Research, 14(7), 994-1007.

Marcus, G., \& Fischer, M. (1986). Anthropology as cultural critique: An experimental movement in the human sciences. Chicago: University of Chicago Press.

Margolis, J. (1978). Persons and minds: The prospects of a nonreductive materialism . Boston: Reider Publishing Company.

Mascolo, M. F. (1994). Toward a social constructivist psychology: The case of selfevaluating emotional development. Journal of Constructivist Psychology, 7, 87-106.

Mason, J. (1996). Qualitative researching. London: Sage.

Maturana, H. R. (1988). Ontology of observing: The biological foundations of selfconsciousness and the physical domain of existence. Conference Workbook: Texts in Cybernetics, American Society for Cybernetics Conference, Felton, CA, 18-23 October.

Maturana, H. R., \& Varela, F. J. (1972/1980). Autopoiesis and cognition: The realization of the living. Boston: Reidel.

Mauthner, M., Birch, M., Jessop, J., \& Miller, T. (2002). Ethics in qualitative research. London: Sage Publications.

McLeod, J. (1997). Narrative and psychotherapy. London: Sage Publications.

McNamee, S. (1994). Research as relationally situated activity: Ethical implications. Journal of Feminist Family Therapy, 6(3), 69-83. 
McNamee, S. (2003a). Bridging incommensurate discourses. Theory and Psychology, 13(3), 387-396.

McNamee, S. (2003b). Bridging incommensurate discourses: A response to Mackay. Theory and Psychology, 13(3), 387-396.

McNamee, S. (2004). Relational bridges between constructionism and constructivism. In J. D. Raskin, \& S. K. Bridges (Eds.), Studies in meaning 2: Bridging the personal and social in constructivist psychology (pp. 37-50). New York: Pace University Press.

McNamee, S., \& Gergen, K. J. (Eds.). (1992). Therapy as social construction. London: Sage Publications.

McWilliams, S. A. (2004). Constructive alternativism and self. In J. D. Raskin, \& S. K. Bridges (Eds.), Studies in meaning 2: Bridging the personal and social in constructivist psychology (pp. 291-309). New York: Pace University Press.

Merleau-Ponty, M. (1945/2003). Phenomenology of perception. London: Routledge.

Miles, M. B., \& Huberman, A. M. (1984). Qualitative data analysis: A sourcebook of new methods. London: Sage.

Miles, M. B., \& Huberman, A. M. (1994). Qualitative data analysis (2nd ed.). Thousand Oaks: Sage.

Mininni, G. (2008). What is it like to be a person? The contribution of discursive psychology to idiographic science. Yearbook of Idiographic Science, 1, 249-272.

Mishler, E. G. (1990). Validation in inquiry-guided research: The role of exemplars in narrative studies. Harvard Educational Review, 60, 415-442.

Mishler, E. G. (1991). Representing discourse: The rhetoric of transcription. Journal of Narrative and Life History, 1(4), 255-280.

Molenaar, P. C. M., \& Valsiner, J. (2008). How generalization works through the single case: A simple idiographic process analysis of an individual psychotherapy. Yearbook of Idiographic Science, 1, 23-38. 
Molenaar, P. C. M. (2004). A manifesto on psychology as idiographic science: Bringing the person back into scientific psychology - this time forever. Measurement: Interdisciplinary Research and Perspectives, 2, 201-218.

Molenaar, P. C. M., Huizinga, H. M., \& Nesselroade, J. R. (2003). The relationship between the structure of inter-individual and intra-individual variability: A theoretical and empirical validation of developmental systems theory. In U. M. Staudinger, \& U. Lindenberger (Eds.), Understanding human development: Dialogues with lifespan psychology (pp. 339-380). Massachusetts: Kluwer Academic Publishers.

Morse, J. (1999a). Myth \#93: Reliability and validity are not relevant to qualitative inquiry. Qualitative Health Research, 9, 717-718.

Neimeyer, R. A. (1985). Problems and prospects in personal construct theory. In D. Bannister (Ed.), Issues and approaches in personal construct theory (pp. 143-171). London: Academic Press.

Neimeyer, R. A. (1993). An appraisal of constructivist psychotherapies. Journal of Constructivist Psychology, 61(2), 221-234.

Neimeyer, R. A. (1993a). Constructivist approaches to the measurement of meaning. In G. J. Neimeyer (Ed.), Constructivist assessment (pp. 58-103). California: Sage.

Neimeyer, R. A. (1994). The role of client-generated narratives in psychotherapy. Journal of Constructivist Psychology, 7, 229-242.

Neimeyer, R. A. (1995). Client-generated narratives in psychotherapy. In R. A. Neimeyer \& M. J. Mahoney (Eds.), Constructivism in Psychotherapy. (pp. 231246). Washington, DC: American Psychological Assosciation.

Neimeyer, R. A. (2000). Narrative disruptions in the construction of the self. In R. A. Neimeyer, \& J. D. Raskin (Eds.), Constructions of disorder: Meaning-making frameworks for psychotherapy (pp. 207-242). Washington, DC: American Psychological Association.

Neimeyer, R. A. (2001). Reauthoring life narratives: Grief therapy as meaning reconstruction. Israel Journal of Psychiatry and Related Sciences, 38, 171-183. 
Neimeyer, R. A. (2009). Constructivist psychotherapy. London: Routledge.

Neimeyer, R. A., Anderson, A., \& Stockton, L. (2001). Snakes versus ladders: A validation of laddering technique as a measure of hierarchical structure. Journal of Constructivist Psychology, 14, 85-106.

Neimeyer, R. A., \& Jackson, T. T. (1997). George A. Kelly and the development of personal construct theory. In W. Bringmann, H. Luck, R. Miller \& C. Early (Eds.), A pictorial history of psychology. Illinois: Quintessence.

Neimeyer, R. A., \& Levitt, H. M. (2000). What's narrative got to do with it? Construction and coherence in accounts of loss. In J. H. Harvey \& E. D. Miller (Eds.), Loss and trauma: general and close relationship perspectives (pp. 401-412). New York: Taylor \& Francis Group.

Neimeyer, R. A., \& Stewart, A. E. (2000). Constructivist and narrative psychotherapies. In C. R. Snyder, \& R. E. Ingram (Eds.), Handbook of psychological change: Psychotherapy processes \& practice for the 21 st century (pp. 337-357). New York: Wiley.

Nesselroade, J. R., Gerstorf, D., Hardy, S. A., \& Ram, N. (2007). Idiographic filters for psychological constructs. Measurement, 5(4), 217-235.

Noaparast, K. B. (1995). Toward a more realistic constructivism. Advances in Personal Construct Psychology, 3, 37-59.

Noaparast, K. B. (2000). Construct and words. Constructivism in the Human Sciences, 5(5), $65-70$

Noaparast, K. B. \& Khosravi, Z. (2006). Mind and mental health base on realistic constructivism. Constructivism in the Human Sciences, 11(1), 20-31.

Nunkoosing, K. (2005). The problem with interviews. Qualitative Health Research, 15(5), 698-706.

Palmer, R. (1969). Hermeneutics: Interpretation theory in Schleiermacher, Dilthey, Heidegger and Gadamer. Evanston: Northwestern University Press. 
Parker, I. (1999). Against relativism in psychology, on balance. History of the Human Sciences, 12, 61-78.

Parker, I. (2005). Qualitative psychology: Introducing radical research. Maidenhead, England: Open University Press.

Perrault, C. (1889). Histoires ou contes de tems passe, avec de moralites: Contes de ma mere l'oye [Fairy tales of times past, with morals: Mother goose tales.]. In A. Lang (Ed.), The blue fairy book (pp. 51-53). London: Dover Publications.

Poland, B. D. (2001). Transcription quality. In J. Gubrium, \& J. Holstein (Eds.), Handbook of interview research: Context and method (pp. 629-649). Thousand Oaks: Sage Publications.

Poland, B. D. (1995). Transcription quality as an aspect of rigor in qualitative research. Qualitative Inquiry, 1, 290-310.

Poland, B. D. (2001). Transcription quality. In J. F. Gubrium, \& J. A. Holstein (Eds.), Handbook of interview research: Context and method. (pp. 629-649). Thousand Oaks: Sage Publications.

Poland, B. D. (2003). Transcription quality. In J. A. Holstein, \& J. F. Gubrium (Eds.), Inside interviewing: New lenses, new concerns (pp. 267-289). Thousand Oaks: Sage.

Ponterotto, J. G. (2005). Qualitative research in counseling psychology: A primer on research paradigms and philosophy of science. Journal of Counseling Psychology, 52, 126-136.

Popper, K. (1959). The logic of scientific discovery. New York: Basic Books.

Potter, J. (1996). Representing reality: Discourse, rhetoric and social construction. London: Sage Publications.

Potter, J. (1998). Fragments in the realization of relativism. In I. Parker (Ed.), Social constructionism, discourse, and realism (pp. 27-46). London: Sage Publications.

Proctor, H. G. (2009). The construct. In R. Butler (Ed.), Reflections in personal construct theory (pp. 21-40). Chichester: John Wiley \& Sons. 
Proctor, H. G. (2011). The roots of Kellian notions of philosophy: The categorical philosophers - Kane, Hegel and Peirce. In D. Stojnov, V. Dzinovic, \& M. Frances (Eds.), Personal consruct psychology in an accelerating world (pp. 29-46). Belgrade, Serbia: Serbian Constructivist Association, EPCA.

Psathas, G., \& Anderson, T. (1990). The 'practices' of transcription in conventional analysis. Semiotica, 78, 75-99.

Pyett, P. M. (2003). Validation of qualitative research in the 'real world'. Qualitative Health Research, 13(8), 1170-1179.

Quine, W. V. (1960). Word and object. Cambridge, Massachusetts: MIT Press.

Radley, A., \& Chamberlain, K. (2001). Health psychology and the study of the case: From method to analytic concern. Social Science and Medicine, 53(3), 321-332.

Raskin, J. D. (2001). On relativism in constructivist psychology. Journal of Constructivist Psychology, 14, 285-313.

Raskin, J. D. (2002). Constructivism in psychology: Personal construct psychology, radical constructivism, and social constructionism. In J. D. Raskin, \& S. K. Bridges (Eds.), Studies in meaning: Exploring constructivist psychology (pp. 1-25). New York: Pace University Press.

Raskin, J. D. (2008). The evolution of constructivism. Journal of Constructivist Psychology, $21,1-24$.

Raskin, J. D. (2011). On essences in constructivist psychology. Journal of Theoretical and Philosophical Psychology, 31(4), 223-239.

Raskin, J. D., \& Bridges, S.K. (2004). Studies in meaning: Bridging the personal and social in constructivisy psychology. New York: Pace University Press.

Raskin, J. D., \& Neimeyer, R. A. (2003). Coherent constructivism: A response to Mackay. Theory and Psychology, 13(3), 397-409.

Rescher, N. (1987). Scientific realism. Boston: Reidel Publishing Company. 
Richardson, L. (1994). Writing: A method of inquiry. In N. Denzin, \& Y. Lincoln (Eds.), Handbook of qualitative research (pp. 516-529). London: Sage Publishing.

Richardson, L. (2000). New writing practices in qualitative research. Sociology of Sport Journal, 17, 5-20.

Rorty, R. (1979/2009). Philosophy and the mirror of nature (Thirtieth Anniversary Edition). Oxfordshire: Princeton University Press.

Rosaldo, R. (1993). Culture and truth: The remaking of social analysis. London: Routledge.

Rowe, D. (1978). The experience of depression. Chichester, England: Wiley.

Rowe, D. (1993). The importance of personal construct psychology. In B. M. Walker, J. Costigan, L. L. Viney \& B. Warren (Eds.), Personal construct theory: A psychology for the future. Melbourne, Australia: Australian Psychological Society.

Rowe, D. (2003). Personal construct psychology and me. In International handbook of personal construct psychology (pp. 397-404). Chichester, England: John Wiley and Sons.

Russel, B. (1985). The philosophy of logical atomism. Ca Salle: Open Court.

Ryan, G. W., \& Bernard, H. R. (2003). Techniques to identify themes. Field Methods, 15(1), 85-109.

Rychlak, J. F. (1990). George Kelly and the concept of construction. International Journal of Personal Construct Psychology, 3, 7-20.

Salvatore, S., Tebaldi, C., \& Poti, S. (2008). The discursive dynamic of sensemaking. Yearbook of Idiographic Science, 39-74.

Salvatore, S., \& Valsiner, J. (2008). Idiographic science on its way: Towards making sense of psychology. Yearbook of Idiographic Science, 9-22.

Sandelowski, M. (2006). "Meta-jeopardy": The crisis of representation in qualitative metasynthesis. Nursing Outlook, 54(1), 10-16. 
Scheer, J. (2010). Computer programs for the analysis of repertory grids. Retrieved March, 2010, from http://www.pcp-net.de/info/comp-prog.html

Scheer, J. W. (2000). The person in society: Challenges to a constructivist theory. Giessen, Germany: Psychosozial-Verlag.

Scheurich, J. (1995). A postmodern critique of research interviewing. Qualitative Studies in Education, 8, 239-252.

Scheurich, J. (1997). Research methods in the postmodern. London: Routledge Falmer.

Scheurich, J. (2003). The masks of validity: A deconstructive investigation. In I. F. Goodson, \& J. Scheurich (Eds.), Research methods in the postmodern (Qualitative Studies Series: 3rd ed., pp. 80-93). London: Routledge Falmer.

Scheurich, J. J. (1996). The mask of validity: A deconstructive investigation. International Journal of Qualitative Studies in Education, 9(1), 49-60.

Schwartz-Shea, P. (2006). Judging quality: Evaluative criteria and epistemic communities. In (pp. 89-114). New York: M.E. Sharpe.

Seale, C., Gobo, C., Gubrium, J. F., \& Silverman, D. (2004). Introduction: Inside qualitative research. In C. Seale, C. Gobo, J. F. Gubrium \& D. Silverman (Eds.), Qualitative research practice. Thousand Oaks: Sage.

Searle, J. R. (1995). The construction of social reality. Harmondsworth: Penguin.

Searle, J. R. (1999). Mind, language and society: Philosophy in the real world. New York: Basic Books.

Sennett, R. (1977). The fall of public man. London: Cox and Wyman.

Shaw, M. L. G. (1979). Conversational heuristic for eliciting shared understanding. International Journal of Man-Machine Studies, 11(5), 621-634.

Shin, K. R., Kim, M. Y., \& Chung, S. E. (2009). Methods and strategies utilized in published qualitative research. Qualitative Health Research, 19(6), 850-858. 
Silverman, D. (1993). Interpreting qualitative data. London: Sage.

Silverman, D. (1993). The machinery of interaction: Remaking social science. The Sociological Review, 41(4), 731-752.

Silverman, D. (2000). Doing qualitative research. London: Sage.

Silverman, D. (2006). Interpreting qualitative data: Methods for analyzing talk, text and interaction (3rd ed.). London: Sage Publications.

Silverman, D. (2009). Doing qualitative research (3rd ed.). London: Sage.

Sin, C. (2003). Interviewing in "place": The socio-spatial construction of interview data. AREA, 35(3), 305-312.

Smith, B., \& Sparkes, A. (2008). Contrasting perspectives on narrating selves and identities: An invitation to dialogue. Qualitative Research, 8(1), 5-35.

Smith, B., \& Sparkes, A. (2009). Narrative inquiry in sport and exercise psychology: What can it mean, and might we do it? Psychology of Sport and Exercise, 10, 1-11.

Smith, J. (1984). The problem of criteria for judging interpretive inquiry. Educational Evaluation and Policy, 6, 379-391.

Smith, J. (1989). The nature of social and educational inquiry: Empiricism versus interpretation. Norwood, New Jersey: Ablex.

Smith, J. (1993). After the demise of empiricism: The problem of judging social and educational inquiry. New Jersey: Ablex Publishing Corporation.

Smith, J. (1997). The stories educational researchers tell about themselves. Educational Researcher, 26, 4-11.

Smith, J., \& Deemer, D. (2000). The problem of criteria in the age of relativism. In N. Denzin, \& Y. S. Lincoln (Eds.), Handbook of qualitative research (2nd ed., pp. 877896). Thousand Oaks: Sage. 
Smith, J., Harre, R., \& van Langenhove. (1995). Rethinking methods in psychology. London, Thousand Oaks: Sage Publications.

Smith, J., \& Hodkinson, P. (2005). Relativism, criteria and politics. In N. Denzin, \& Y. S. Lincoln (Eds.), The Sage handbook of qualitative research (3rd ed., pp. 915-932). Thousand Oaks, London: Sage.

Smith, J., \& Hodkinson, P. (2009). Challenging neorealism: A response to Hammersley. Qualitative Inquiry, 15(1), 30-39.

Smith, J. A. (1990). Alternative research paradigms and the problem of criteria. In E. Guba (Ed.), The paradigm dialog (pp. 167-187). London: Sage.

Smith, J. A. (1996a). Beyond the divide between cognition and discourse: Using interpretive phenomenological analysis in health psychology. Psychology and Health, 11, 261-271.

Smith, J. A. (1996b). Evolving issues for qualitative psychology. In J. Richardson (Ed.), Handbook of qualitative research methods (pp. 189-202). Leicester: BPS.

Smith, J. A. (2003). The origins of qualitative psychology. In J. A. Smith (Ed.), Qualitative psychology: A practical guide to research methods (pp. 4-25). London: Sage.

Smith, J. A. (2003). Shifting identities: The negotiation of meanings between texts and between persons. In L. Finlay, \& B. Gough (Eds.), Doing reflexivity (pp. 176-186). Oxford: Blackwell Publishing.

Smith, J. A. (2004). Reflecting on the development of interpretative phenomenological analysis and its contribution to qualitative research in psychology. Qualitative Research in Psychology, 1, 39-54.

Smith, J. A. (Ed.). (2008). Qualitative psychology: A practical guide to research methods (2nd ed.). Thousand Oaks: Sage.

Smith, J. A., Flowers, P., \& Larkin, M. (2009). Interpretive phenomenological analysis: Theory, method and research. London: Sage Publications. 
Smith, J. A., Harre, R., \& van Langenhove, L. (1996). Idiography and the case-study. In Rethinking psychology (Smith, J.A.; Harre, R.; van Langenhove, L. ed., pp. 59-69). London: Sage Publications.

Smythe, W. E., \& Murray, M. J. (2000). Owning the story: Ethical considerations in narrative research. Ethics and Behavior, 10(4), 311-336.

Solas, J. (1992). Ideological dimensions implicit in Kelly's theory of personal constructs. International Journal of Personal Construct Psychology, 5, 377-392.

Solas, J. (1995). Grammatology of social construing. In R. A. Neimeyer, \& G. J. Neimeyer (Eds.), Advances in personal construct psychology (pp. 61-75). London: Academic Press.

Sparkes, A. (1995). Writing people: Reflections on the dual crisis of representation and legitimation in qualitative inquiry. QUEST: Advancing Kinesiology in Higher Education, 47(2), 158-195.

Sparkes, A. (2001). Myth 94: Qualitative health researchers will agree about validity. Qualitative Health Research, 11(4), 538-552.

Sparkes, A. (2002). Authethnography: Self-indulgence or something more? In A. P. Bochner, \& C. Ellis (Eds.), Ethnographically speaking: Autoethnography, literature, and aesthetics (pp. 209-232). Oxford: Alta Maria Press.

Sparkes, A. (2003a). From performance to impairment: A patchwork of embodied memories. In J. Evans, B. Davies \& J. Wright (Eds.), Body knowledge and control memories (pp. 157-172). London: Routledge.

Sparkes, A. (2003b). Bodies, identities, selves: Autoethnographic fragments and reflections. In J. Denison, \& P. Markula (Eds.), Moving writing: Crafting movement in sport research (pp. 51-76). New York: Peter Lang.

Sparkes, A., \& Smith, B. (2009). Judging the quality of qualitative inquiry: Criteriology and relativism in action. Psychology of Sport and Exercise, 10, 491-497. 
Stake, R. (1994). Case studies. In N. Denzin, \& Y. Lincoln (Eds.), Handbook of qualitative research (pp. 236-247). Thousand Oaks: Sage.

Stevens, C. D. (1998). Realism and Kelly's pragmatic constructivism. Journal of Constructivist Psychology, 1, 283-308.

Stojnov, D., \& Butt, T. (2002). The relational basis of personal construct psychology. In R. Neimeyer, \& G. Neimeyer (Eds.), Advances in personal construct psychology: New directions and perspectives (pp. 81-112). London: Praeger Publishing.

Strathern, M. (1990). The gender of the gift. Berkley, California: University of California Press.

Strauss, A., \& Corbin, J. (1990). Basics of qualitative research: Guided theory procedures and techniques. Thousand Oaks: Sage Publications.

Swenson, R. (1992). Autocatakinetics, yes - autopoiesis, no: Steps toward a unified theory of evolutionary ordering. International Journal of General Systems, 21(2), 207-228.

Talburt, S. (2004). Ethnographic responsibility without the real. The Journal of Higher Education, 75(1), 80-103.

Tarski, A. (1944). The semantic conception of truth and the foundations of semantics. Philosophy and Phenomenology Research, 4, 341-375.

Taylor, C. (2006). Source of the self: The making of the modern identity. Cambridge: Cambridge University Press.

Teddlie, C., \& Tashakkori, A. (2003). Major issues and controversies in the use of mixed methods in the social and behavioural sciences. In A. Tashakkori, \& C. Teddlie (Eds.), Handbook of mixed-methods in social and behavioural research (pp. 3-50). Thousand Oaks: Sage Publications.

Tierney, W. (1993). The cedar closet. International Journal of Qualitative Studies in Education, 6(4), 303-314. 
Tilley, S. A. (2003a). "Challenging" research practices: Turning a critical lens on the work of transcription. Qualitative Inquiry, 9(5), 750-773.

Tilley, S. A. (2003b). Transcription work: Learning through coparticipation in research practices. Qualitative Studies in Education, (16), 6-835.

Tsang, T. (2000). Let me tell you a story: A narrative exploration of identity in highperformance sport. Sociology of Sport Journal, 17, 44-59.

Tschudi, F. (1983). Constructs are hypotheses. In J. Adams-Webber, \& J. C. Mancuso (Eds.), Applications of personal construct psychology (pp. 115-126). London: Academic Press.

Vaihinger, H. (1925/1952). The philosophy of 'as if': A system of the theoretical, practical and religious fictions of mankind (C. K. Ogden Trans.). London: Harcourt Brace \& Company.

Valsiner, J., \& Sato, T. (2006). Historically structured sampling (HSS): How can psychology's methodology become tuned into the reality of the historical nature of cultural psychology? In J. Straub, C. Kolbl, D. Weidemann \& B. Zielke (Eds.), (pp. 215-251). Bielefeld, Germany: Transkript.

van Maanen, J. (2011). Tales of the field: On writing ethnography (2nd ed.). Chicago: University of Chicago Press.

van Manen, M. (1990). Researching lived experience: Human science for an action sensitive pedagogy. Ontario, Canada: The Althouse Press.

Viney, L. (1987). Interpreting the interpreters. Florida: Robert Krieger Publishing Co.

Viney, L. (1988). Which data-collection methods are appropriate for a constructivist psychology. International Journal of Personal Construct Psychology, 1, 191-203.

Viney, L. (1993). Life stories. London: Wiley.

Wachterhauser, B. (2002). Getting it right: Relativism, realism and truth. In R. Dostal (Ed.), The cambridge companion to Gadamer (pp. 52-78). Cape Town, South Africa: Cambridge University Press. 
Walker, B. M. (1990). Construing George Kelly's construing of the person-in-relation. International Journal of Personal Construct Psychology, 3, 41-50.

Warren, B. (1990). Psychoanalysis and personal construct theory: An exploration. Journal of Psychology, 124, 449-464.

Warren, B. (2004). Construing constructionism: Some reflections on the tension between PCP and social constructionism. Personal Construct Theory and Practice, 1, 33-44.

Warren, W. G. (1985). Personal construct psychology and contemporary philosophy: An examination of alignments. In D. Bannister (Ed.), Issues and approaches in personal construct theory. (pp. 253-265). London: Academic Press.

Warren, W. G. (1989). Personal construct theory and general trends in contemporary philosophy. International Journal of Personal Construct Psychology, 2(3), 287-300.

Warren, W. G. (1991). Rising up from down under: A response to Adams-Webber on cognitive psychology and personal construct theory. Journal of Personal Construct Psychology, 4(1), 43-49.

Warren, W. G. (1992). Subjecting and objecting in personal construct psychology. In A. Thomson, \& P. Cummins (Eds.), European perspectives in personal construct psychology (pp. 57-66). Lincoln, Nebraska: European Personal Construct Association.

Warren, W. G. (1997). Refocussing the subject: The anarcho-psychological tradition revisited. Educational Philosophy and Theory, 28(1), 89-196.

Warren, W. G. (1998). Philosophical dimensions of personal constructs psychology. London: Routledge, Taylor and Francis Group.

Warren, W. G. (2000). Personal construct psychology, neostructuralism and hermeneutics. In J. W. Scheer (Ed.), The person in society: Challenges to a constructivist theory (pp. 7889). Giessen, Germany: Psychosozial-Verlag.

Warren, W. G. (2003). Pragmatism and religion: Dewey's twin influences. In (pp. 387-396). Chichester, England: John Wiley and Sons. 
Warren, W. G. (2010). Kelly’s personal construct psychology and Dewey’s pragmatism: some direct and some 'intellectual context' aspects. Personal Construct Theory and Practice, 7, 32-40.

Watson, C. (2006). Unreliable narrators? Inconsistency (and some inconstancy) in interviews. Qualitative Research, 6(3), 367-384.

Wengraf, T. (2001). Qualitative research interviewing: Biographic narrative and semistructured methods. London, Thousand Oaks: Sage Publications.

Whittemore, R., Chase, S. K., \& \& Mandle, C. L. (2001). Pearls, pith, and provocation: Validity in qualitative research. Qualitative Health Research, 11(4), 522-537.

Wittgenstein, L. (1958/1972). Primary studies for the 'philosophical investigations' generally known as the blue brown books. Oxford: Blackwell.

Wittgenstein, L. (1968). Philosophical investigations (C. K. Ogden Trans.). London: Routledge \& Kegan Paul.

Wittgenstein, L. (1922/1999). Tractatus logico-philosophicus (C. K. Ogden Trans.). New York: Routledge.

Wolcott, H. (1994). Transforming qualitative data: Description, analysis, and interpretation. London: Sage.

Wolcott, H. F. (1990). On seeking-and rejecting-validity in qualitative research. In E. W. Eisner, \& A. Peshkin (Eds.), Qualitative inquiry in education: The continuing debate (pp. 121-152). New York: Teachers College Press.

Wolcott, H. F. (1995). Making a study more ethnographic. In J. van Maanen (Ed.), Representation in ethnography (pp. 79-111). London: Sage.

Wolcott, H. F. (1999). Ethnography: A way of seeing. Oxford: The Rowman \& Littlefield Publishing Group.

Wolcott, H. F. (2005). The art of fieldwork. Oxford: The Rowman \& Littlefield Publishing Group. 
Wortham, S. (1996). Are constructs personal? Theory and Psychology, 6(1), 79-84.

Wright, E. (Ed.). (2008). The case for qualia. Cambridge, Massachusetts: MIT Press.

Yanos, P., \& Hopper, K. (2008). On 'false, collusive objectification': Becoming attuned to self-censorship, performance and interviewer biases in qualitative research. International Journal of Social Research Methodology, 11(3), 229-237.

Yanow, D., \& Schwartz-Shea, P. (Eds.). (2006). Interpretation and method: Empirical research methods and the interpretive turn. New York: M.E. Sharpe.

Yardley, L. (2000). Dilemmas in qualitative health research. Psychology and Health, 15, 215228.

Yardley, L. (2008). Demonstrating validity in qualitative psychology. In J. A. Smith (Ed.), Qualitative psychology: A practical guide to research methods (2nd ed., pp. 235-251). Thousand Oaks: Sage Publications.

Yin, R. K. (1994). Case study research: Design and methods (2nd ed.). Thousand Oaks: Sage Publications.

Yin, R. K. (2004). The case study anthology. Thousand Oaks: Sage Publications.

Yin, R. K. (2009). Case study research: Design and methods (4th ed.). California: Sage.

Yorke, M. (1989). The intolerable wrestle: Words, numbers, and meanings. International Journal of Personal Construct Psychology, 2, 65-76.

Yorke, M. (2001). Bipolarity...or not? some conceptual problems relating to bipolar rating scales. British Educational Research Journal, 27(2), 171-186. 